Old Dominion University

ODU Digital Commons

Physics Theses \& Dissertations

Physics

Summer 2010

\title{
Feasibility and Conceptual Design of a C.W. Positron Source at CEBAF
}

Serkan Golge

Old Dominion University

Follow this and additional works at: https://digitalcommons.odu.edu/physics_etds

Part of the Elementary Particles and Fields and String Theory Commons, and the Nuclear Commons

\section{Recommended Citation}

Golge, Serkan. "Feasibility and Conceptual Design of a C.W. Positron Source at CEBAF" (2010). Doctor of Philosophy (PhD), Dissertation, Physics, Old Dominion University, DOI: 10.25777/5z6h-h261

https://digitalcommons.odu.edu/physics_etds/44

This Dissertation is brought to you for free and open access by the Physics at ODU Digital Commons. It has been accepted for inclusion in Physics Theses \& Dissertations by an authorized administrator of ODU Digital Commons. For more information, please contact digitalcommons@odu.edu. 


\title{
FEASIBILITY AND CONCEPTUAL DESIGN OF A C.W. POSITRON SOURCE AT CEBAF
}

\author{
by \\ Serkan Golge \\ B.S. July 2002, Fatih University \\ M.S. December 2004, Old Dominion University \\ A Dissertation Submitted to the Faculty of \\ Old Dominion University in Partial Fulfillment of the \\ Requirement for the Degree of \\ DOCTOR OF PHILOSOPHY \\ PHYSICS \\ OLD DOMINION UNIVERSITY \\ August 2010 \\ Approved by: \\ Charles F. Hvde (Director) \\ Arne P. Freyberger \\ Anatolv V. Radvushkin \\ Moskov Amarvan \\ Sacharia Albin
}




\title{
ABSTRACT \\ FEASIBILITY AND CONCEPTUAL DESIGN OF A C.W. POSITRON SOURCE AT CEBAF
}

\author{
Serkan Golge \\ Old Dominion University, 2010 \\ Director: Dr. Charles E. Hyde
}

A feasibility study of a CW positron source for the $12 \mathrm{GeV}$ upgrade at Jefferson Lab (JLAB) is provided. The proposed $\geq 100 \mathrm{nA}$ Continuous Wave (CW) positron source at JLAB has several unique and challenging characteristics: high current incident electron beam at $126 \mathrm{MeV}$ with a high beam power (up to a $\mathrm{MW}$ ); $\mathrm{CW} \mathrm{e}^{-}$ beam and $\mathrm{CW} \mathrm{e}^{+}$production. The multiple scattering is a dominant process when creating $\mathrm{e}^{+}$in a target, which results a large phase space area of the emitted positrons. An admittance study was done at CEBAF to find the maximum phase space area, which is tolerated in the machine. The measured geometrical transverse admittance (A) were $A_{x}=10$ and $A_{y}=5 \mathrm{~mm} \cdot \mathrm{mrad}$ at the injector. Energy spread measurement was also done at the ARC1. The fractional spread limit in the ARC1 was measured as $\delta=3 \times 10^{-3}$ at $653 \mathrm{MeV}$. By using the optimized results and the CEBAF parameters, three positron injector configurations are proposed; Combined Function Magnet, Two-Dipole and Microtron Dipole configurations. With the assumptions made, by using $126 \mathrm{MeV} \otimes 10 \mathrm{~mA} \mathrm{e} \mathrm{e}^{-}$beam impinging on a $2 \mathrm{~mm}$ W target with a $100 \mu \mathrm{m}$ spot size, we can get up to $3 \mu \mathrm{A}$ useful $\mathrm{e}^{+}$current at the North Linac connection. One of the biggest challenges is the target design, which the deposited power is about $60 \mathrm{~kW}$. ILC designs project power deposition up to $13 \mathrm{~kW}$, which would allow the creation of a $\mathrm{e}^{+}$beam of up to $650 \mathrm{nA}$ otherwise. The results of analytic and monte carlo simulations of the positron production, capture and acceleration are presented. For the target design, a review is presented of solutions for the high power production target. Portions of this dissertation work have been published in two conference proceedings. ${ }^{1,2}$

\footnotetext{
${ }^{1}$ S. Golge et. al., in Proceedings of PAC07, Albuquerque, New Mexico, June 2007

${ }^{2}$ S. Golge et. al., AIP Conf. Proc., 1160, 109 (2009)
} 
(C)Copyright, 2010, by Serkan Golge, All Rights Reserved 
To my father Mustafa Golge, 


\section{ACKNOWLEDGMENTS}

I really wish that I could thank every person in my life who directly or indirectly helped me to finish this work. Nevertheless, I will be able to mention only a few of them who have been closer to me. From my personal experience, and from my observation of other candidates' experiences, I realized that it takes great responsibility and a significant commitment to mentor someone during his or her doctoral candidacy. I consider myself very fortunate that I have had two distinguished mentors who not only guided me in my scientific career, but also inspired me to build a better character as a scientist and as a productive member of society. I would like to express my sincere gratitude to Dr. Charles Hyde and Dr. Arne Freyberger for their unmatched help, patience and friendship throughout my study. I thank Dr. Hyde for putting his trust in me from the very beginning until the very end. He taught me how to ask the right questions and challenge any obstacle in my way with an undiminished courage. Dr. Freyberger has excellent skills, which have assisted me in finishing months of work in days. I am grateful to him for helping me to unleash these skills and become more efficient in my academic studies.

I would like to thank my committee members, Dr. Anatoly Radyushkin, Dr. Moskov Amaryan and Dr. Sacharia Albin for their valuable time and discussions, which helped me bring this dissertation to a higher level.

I am grateful that I have met with outstanding and very professional faculty and staff members at Old Dominion University. I would like to thank Physics department chair Dr. Gail Dodge as a representative of all the faculty and administrative staff members of the department.

It is my pleasure to have closely worked with one of the most respected scientific communities at Jefferson Lab. The Center for Advanced Accelerator Studies members have always been available to answer my questions. I am indebted to Dr. Geoffrey Krafft who has been an exceptional teacher and a mentor to me. I thank his wife, Alicia Hofler, and wish them a blessed future. I thank Dr. Reza Kazimi, Dr. Joe Grames, Dr. Alex Bogacz, Dr. Jay Benesch, Dr. Kevin Beard, Dr. David Douglas, Dr. Tony Forest, Dr. Eric Voutier and Dr. Hari Areti for their continuous support. This dissertation could not have been written without them.

I thank my colleagues in the Physics department for their fruitful discussions and for their heartfelt friendship. I thank all of them, especially Dr. Salim Balik who 
encouraged me to choose Old Dominion University (ODU) for my graduate study and my colleagues and former roommates Islam Bedir, Mustafa Canan and Oguz Er. With all of them, I have experienced joyful moments in my years at ODU. I also thank my fellow graduate students Bayram Torayev, Hisham Sayed, Donika Plyku, Dr. Eman Ahmed, Dr. Giovanni Chirilli, Dr. Jixie Zhang, Dr. Saori Pastore, Dr. Nevzat Guler, Dr. Janette Drake, Dr. Megh Niroula, Krishna Adhikari, Marija Raskovic and all my other friends.

I am indebted to all faculty members at Fatih University who were part of building my academic career.

Any attempt to thank my parents will not be sufficient, but I will still try and say that I am grateful to have such wonderful parents, my mother Emel and my father Mustafa Golge. Their sacrifices and unwavering care and support for me will never be forgotten. I will always try to be an excellent parent like them. I also thank my sisters Nuriye Dilek and Zeynep Dilber and my little brother Furkan for being supportive of me all the time.

Another unsuccessful attempt to express my gratitude will be to my wife, Kubra. She has been supportive of me all the time. She has shown a tremendous effort to prevent any distraction and only to enable me to focus on my work. Without her I would not and cannot finish my studies.

I would like to thank all of my relatives, friends and everyone who has helped and guided me through my life. Please know that limits on pages prevent me writing your names but nothing can make your names disappear from my heart. Thank you to all of you. 


\section{TABLE OF CONTENTS}

List of Tables . . . . . . . . . . . . . . . . . . ix

List of Figures . . . . . . . . . . . . . . . . $x$

Chapter

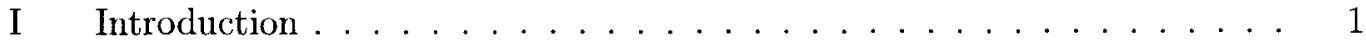

I.1 Basic Accelerator Physics and Definitions . . . . . . . . . . . 4

I.1.1 Equation of Motion . . . . . . . . . . . . . . . 4

I.1.2 Thin lens approximation . . . . . . . . . . . 10

I.1.3 Phase ellipse and transverse emittance . . . . . . . . . 11

I.1.4 Dispersion Function . . . . . . . . . . . . . . . . 17

I.1.5 Achromat . . . . . . . . . . . . . . . . . . . 19

I.2 Definitions . . . . . . . . . . . . . . . . . . 22

I.3 Basic Description of CEBAF . . . . . . . . . . . 26

I.4 Simulation Tools . . . . . . . . . . . . . . . . . 30

II Positron Sources . . . . . . . . . . . . . . . . . . . . . . . . . 38

II.1 Non-accelerator Positron Sources . . . . . . . . . . . . . . . . . 39

II.2 Review of Accelerator Based Positron Sources . . . . . . . . . . . . 40

II.2.1 Stanford Linear Accelerator Center (SLAC) . . . . . . . . . 40

II.2.2 Beijing Electron Positron Collider (BEPCII) . . . . . . . . . . 46

II.2.3 Cornell Electron Storage Ring (CESR) . . . . . . . . . . . . 47

II.2.4 The Budker Institute of Nuclear Physics (VEPP) . . . . . . 50

II.2.5 International Linear Collider (ILC) . . . . . . . . . . . 51

II.2.6 Conclusion . . . . . . . . . . . . . . . . . . . . . . 53

III Admittance Measurement at CEBAF . . . . . . . . . . . . . . . 55

III.1 Transverse Admittance . . . . . . . . . . . . . . . . . . . . . . 55

III.2 Longitudinal Admittance . . . . . . . . . . . . . . . . . . 60

IV Design Options for a Positron Source . . . . . . . . . . . . . 66

IV.1 Optimization ........................ 68

IV.2 Quarter Wave Transformer (QWT) . . . . . . . . . . . . 81

IV.3 Design - 1 : Combined Function Magnets (CFM) . . . . . . . . . . . 84 
IV.4 Design - 2: Two Dipoles . . . . . . . . . . . . . 106

IV.5 Design - 3 : Microtron Dipoles . . . . . . . . . . . . . 126

V Power and Radiation . . . . . . . . . . . . . . . 136

V.1 Power Flow . . . . . . . . . . . . . . . . . . 137

VI Summary and Conclusion . . . . . . . . . . . . . . 143

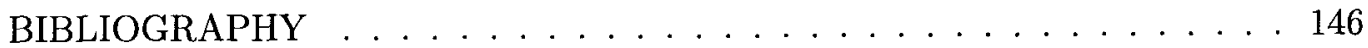

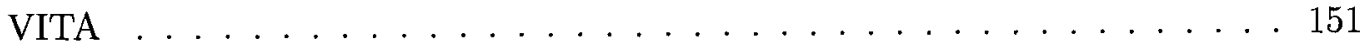




\section{LIST OF TABLES}

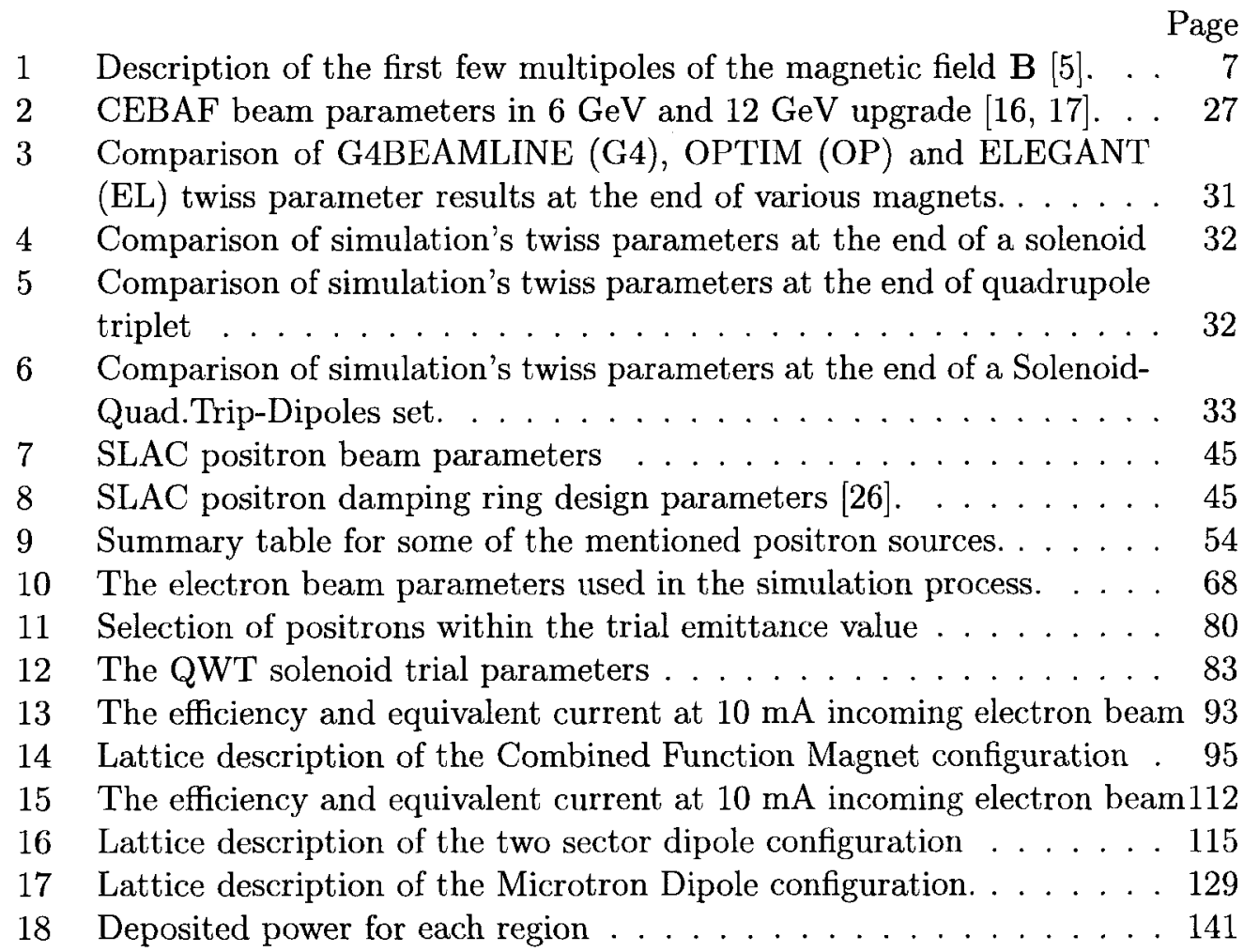




\section{LIST OF FIGURES}

Page

1 The coordinate system showing the ideal trajectory . . . . . . . 5

2 The design momentum $\mathrm{p}$ with with its momentum rigidity $\mathrm{B} \rho \ldots 8$

3 On the left, a particles movement is shown in cartesian coordinate . . 12

4 The phase space ellipse of the beam representing the area . . . . . . 14

5 Transformation of the $x-x^{\prime}$ phase space ellipse is shown . . . . . 17

6 The dispersion function $\eta$ in a DBA lattice. . . . . . . . . . . 20

7 In this figure [6], micro and macro beam current definitions . . . . . 24

8 The schematic drawing of the CEBAF. . . . . . . . . . 28

9 The original CEBAF $50 \mathrm{~cm}$ effective length 5-cell cavity is shown. . . 29

10 The twiss $\beta$ function plots of Test-6. . . . . . . . . . . . 33

11 The $x-p$ plot of the beam at the end of Test-5 . . . . . . . . 34

12 The $\beta_{x}$ of a non-gaussian flat distribution . . . . . . . . 36

13 Snapshots from the G4 simulation. . . . . . . . . . . . . 37

14 Bremsstrahlung process of an electron when passing by an atomic nuclei 39

15 The schematic layout of the SLAC ring complex . . . . . . . . . . 41

16 PEP-II rings layout where a collision occurs . . . . . . . . . . . 42

17 The SLAC pseudo-adiabatic phase-space transformation system. . . . 43

18 Cross section of the positron converter target in SLAC [28] . . . . . . 44

19 The detailed drawing of the SLAC positron source . . . . . . . . . . 44

20 The schematic layout of the BEPCII positron source. . . . . . . . . 47

21 An isometric view of the focusing coil. . . . . . . . . . . . 48

22 CESR positron converter assembly with focusing solenoid . . . . . . . 49

23 The schematic layout of the VEPP-4 accelerator-ring complex. . . . . 50

24 The helical undulator used to produce photons to create positrons [47]. 52

25 Cross section of the proposed ILC rotating positron converter target assembly [48]. . . . . . . . . . . . . . . . . . . 52

26 An alternative liquid jet target for ILC positron source [47]. . . . . 53

27 The admittance ellipse extending to the walls of the beam pipe [5]. . 56

28 Simple schematic of the location of the CEBAF admittance test. . . . 57

29 Actual scaled schematic of the location of the CEBAF . . . . . . . 58

30 The $\mathrm{H}$ and $\mathrm{V}$ Admittance phase space area in the injector. . . . . . . 59

31 Second set of horizontal data where IPM0R07 and IPM1A39 were used as current monitors. . . . . . . . . . . . . . . . 60

32 The figure shows each set and measured RF cavity . . . . . . . . 62

33 The Beam Energy Status monitor snapshot is shown. . . . . . . . . 63

34 The figure shows each set and measured RF cavity energies . . . . . . 64

35 The Beam Energy Status monitor snapshot . . . . . . . . . . . . 65

36 The momentum distribution of the positrons for different tungsten thicknesses. . . . . . . . . . . . . . . . 69 
37 On the left axis, the conversion efficiency (ratio of all created positrons per incoming number of electrons) as a function of tungsten thickness is shown. . . . . . . . . . . . . . . . .

38 The percentage of the deposited power w.r.t the incoming beam power as a function of the tungsten thickness is plotted . . . . . . . 71

39 The total momentum of the positrons emerging from a $2 \mathrm{~mm}$ tungsten converter . . . . . . . . . . . . . . . . . 72

40 The time $(\mathrm{t})$ distribution of the positrons right after a $2 \mathrm{~mm}$ tungsten target is plotted ...................... 73

$41 p-\theta$ and $p-t$ distributions of the positrons emerging from a $2 \mathrm{~mm}$ tungsten target without any cuts. . . . . . . . . . . 75

$42 x-y$ transverse position distributions of the positrons emerging from a $2 \mathrm{~mm}$ tungsten target without any cuts. . . . . . . . . . 76

$43 x^{\prime}-y^{\prime}$ distributions of the positrons emerging from a $2 \mathrm{~mm}$ tungsten target without any cuts. . . . . . . . . . . 76

$44 x-y$ transverse phase space distributions of the positrons emerging from a $2 \mathrm{~mm}$ tungsten target without any cuts. . . . . . . . . 77

45 The emittance of the positrons from a $2 \mathrm{~mm}$ tungsten . . . . . . . . 78

46 The whole ellipse represents the $100 \%$ of the beam which is the admittance area. . . . . . . . . . . . . . . . . . . 81

47 The short and long solenoidal field profile of the QWT. . . . . . . . 82

48 The acceptance volume of the QWT as a function of central momentum of positrons . . . . . . . . . . . . . . . . . . 84

49 A snapshot from the G4beamline simulation. . . . . . . . . . . 86

50 The simple schematic representation for the proposed service tunnel with CFMs. . . . . . . . . . . . . . . . 87

51 A quick schematic guide to the CFM lattice. . . . . . . . . . . 88

52 The 1- $\sigma_{x y}$ beam sizes of the positron beam as a function of the central orbit are plotted in the CFM lattice . . . . . . . . . . . . . 89

53 The $\beta_{x y}$ functions of the positron beam as a function of the central orbit are plotted in the CFM lattice . . . . . . . . . . . . . . . 90

54 This plot zooms into the first few meters of the CFM lattice. . . . . . 91

55 The full beam envelope of the cut positron beam in transverse $x$. . . 92

56 The full beam envelope of the cut positron beam in transverse $y$. . . 92

57 The positron beam at the end of the CFM lattice . . . . . . . . 94

58 Transverse and longitudinal phase space of the beam after the solenoid in CFM lattice. . . . . . . . . . . . . . . . . . . . . . . . 99

59 The $\mathrm{e}^{+}$beam after the 1st CFM magnet in the CFM lattice. . . . . . 100

60 Transverse and longitudinal phase space of the beam after the 2nd CFM in CFM lattice. . . . . . . . . . . . . . . . . . . . . . . . 101

61 Transverse and longitudinal phase space of the beam right before the 1st quarter cryomodule in the CFM lattice. . . . . . . . . . . . . . 102

62 Transverse and longitudinal phase space of the beam right before the 2nd quarter cryomodule in the CFM lattice. . . . . . . . . . . . 103 
63 Transverse and longitudinal phase space of the beam right after the qC set quad triplet in the CFM lattice . . . . . . . . . . . . . . 104

64 Transverse and longitudinal phase space of the beam right after the full cryomodule in the CFM lattice. . . . . . . . . . . . . . . 105

65 A quick schematic guide to the Two-Dipole lattice. . . . . . . . . . 107

66 A snapshot from the G4beamline simulation . . . . . . . . . . . 108

67 The $1-\sigma$ beam size of the positron beam as a function of the central orbit in Two-Dipole configuration . . . . . . . . . . . . 109

68 The twiss $\beta_{x y}$ functions of the positron beam as a function of the central orbit are plotted in Two-Dipole configuration. . . . . . . . 110

69 This plot zooms into the first few meters of the Two-Dipole lattice . . 111

70 The full beam envelope of the cut positron beam . . . . . . . . . 112

71 The full beam envelope of the cut positron beam . . . . . . . . . . 113

72 The positron beam at the end of the Two-Dipole lattice . . . . . . . . 114

73 Transverse and longitudinal phase space of the beam after the solenoid in Two-Dipole lattice. . . . . . . . . . . . . . . . . . . 119

74 The beam right before the 1st dipole in Two-Dipole lattice. . . . . 120

75 Transverse and longitudinal phase space of the beam right after the 2nd dipole in Two-Dipole lattice . . . . . . . . . . . . . . . . 121

76 Transverse and longitudinal phase space of the beam right after the 1st quarter cryomodule in Two-Dipole lattice . . . . . . . . . . . . . 122

77 Transverse and longitudinal phase space of the beam right after the 2nd quarter cryomodule in Two-Dipole lattice . . . . . . . . . 123

78 Transverse and longitudinal phase space of the beam right before the full cryomodule in Two-Dipole lattice . . . . . . . . . . . . . . . 124

79 Transverse and longitudinal phase space of the beam at the end of the lattice where a connection can be made to the North Linac . . . . . . 125

80 The novel design by using two back to back microtron dipoles . . . 127

81 The $1-\sigma$ beam size of the positron beam as a function of the central orbit in the microtron dipole lattice is plotted . . . . . . . . . 128

82 Top view of a single DP can. . . . . . . . . . . . . . . . . . . 134

83 Three adjacently positioned DP cans immediately before the North Linac in the CEBAF. . . . . . . . . . . . . . . 135

84 Efficiency of the emitted neutron per incident electron $\left(p\left(\mathrm{e}^{-}\right)=126\right.$ $\mathrm{MeV} / \mathrm{c}$ ). The line is drawn to guide the eye only. . . . . . . . . 138

85 The momentum distribution of the electrons in the forward direction 139

86 The momentum distribution profile of the secondary $\gamma$ particles . . 140

87 An alternative liquid jet target for ILC positron source [47]. . . . . 142

88 Cross section of the proposed ILC . . . . . . . . . . . . . . 142 


\section{CHAPTER I}

\section{INTRODUCTION}

Positron (anti-particle of electron), which is a member of the lepton family, has been used for decades for variety of experiments in nuclear physics, solid state, material and biomedical sciences. As an anti-particle, positrons do not exist abundantly in nature and must be created specifically for experiments. High energy physics accelerators widely use positrons for lepton-lepton collision and fixed target experiments. At the Continuous Electron Beam Accelerator Facility (CEBAF) located in Jefferson Laboratory (JLAB), it has also been a desire to produce a positron beam for years $[1,2,3]$. This proposed positron beam will be used for fixed target experiments. We propose to produce a high intensity positron beam with a current $I \geq 100 \mathrm{nA}$ with the transverse and longitudinal emittances to achieve required luminosity requirements for the experimental halls. The applications of a positron beam will help the nuclear physicists advance in many areas such as the Deeply Virtual Compton Scattering (DVCS) program; precision measurements of two-photon effects in high energy electron scattering, etc. The created positron spectrum is so broad that it could also be considered for the possibility of producing intense low energy positrons to study on imaging of materials, surfaces and macro-molecules. The proposed design is; compact in size with respect to other similar scale laboratories; does not require a damping ring and is a unique Continuous Wave (CW) source.

In this dissertation, the requirements for such a beam, challenges and problems for this project is outlined. The main subject and track of this study are focussed on obtaining a solution tailored to the system requirements of CEBAF. Some of these challenges require an extensive engineering $R \& D$ work, and for this, a short guidance is provided including references and proposed solutions from the literature.

In Chapter I, an introduction about the motivation behind this study and outline of this thesis is given. The basic accelerator physics concepts are briefly provided. The complexity of the positron creation process, post-creation, capture and transportation of this positron beam requires utilization of different types of simulation codes and packages. Monte-Carlo based simulation codes to simulate positron creation; lattice design softwares capable of calculating linear and non-linear optics

This dissertation follows the style of Physical Review Special Topics-Accelerators and Beams as journal model. 
parameters have been used. A comparison study between these softwares concludes this chapter.

A very important part of a conceptual design study is to investigate the features and designs of other similar institutions and projects. There are numerous small and large laboratories around the world utilizing positrons for their experimental needs. Some of the most prominent laboratories are introduced in Chapter II. There are also future accelerator projects intending to use positrons. A table comparing the achieved and proposed energies, beam currents between these existing/projected is given to conclude this chapter.

In Chapter III, the results of an admittance (acceptance) study is provided. This study was done at the injector and ARC1 sections of the CEBAF. In accelerators, the positrons are usually created via secondary processes when the electrons pass through a preferably high atomic number material. Due to the multiple scattering, this creates a large phase space distribution of the emitted positrons. As it was mentioned before, this poses a big challenge for the strict restrictions of CEBAF. Our study intended to find the boundaries of the machine since the transported positron beam will not have the same high quality as such as the electron beam. This admittance study consists of two parts. First study measured the transverse acceptance of the machine in the injector section until the North Linac section. The second study is done to measure the transverse and longitudinal acceptance of the North Linac and the ARC1.

The design solutions are introduced in Chapter IV, where the results of optimization study of target parameters and emitted positrons from the target are introduced. Then by taking this optimized result as a starting point and using the results of the admittance study we offer three design considerations in this chapter. The lattice parameters, transverse and longitudinal phase space graphs and betatron functions through the lattices are provided in this chapter.

In Chapter V, one of the main challenges of this project is outlined. The incoming electron beam to create positrons has a very small spot size (on the order of 100 $\mu \mathrm{m}$ ) at an energy of $126 \mathrm{MeV}$ (this is the upgraded injector energy at $12 \mathrm{GeV}$ upgrade of CEBAF) with a high current (10 $\mathrm{mA}$ and higher). This beam poses both advantages and disadvantages to us. The advantages are; well-defined beam emittance, accurate adjustment to a small spot size, CW operation with this high current. At the same time the very same features introduces mechanical challenges 
causing disadvantage for the target; small spot size with this $1 \mathrm{MW}$ incoming beam power will put enormous power on the target which may result in cracking, burning a hole or even melting the converter target. The general solution is that the target must be cooled efficiently and very quickly without sacrificing the output beam quality. There are different methods in the literature. Some of these methods are briefly provided in this chapter.

Final chapter, Chapter VI, concludes this dissertation, by summarizing what has been completed, and gives a brief guidance about what needs to be done for further studies. 


\section{I.1 BASIC ACCELERATOR PHYSICS AND DEFINITIONS}

This section aims to be an introduction to the accelerator physics and beam dynamics. This section was prepared with the help of my lecture notes from graduate courses as well as the books given in the following references $[4,5,6,7]$. Throughout the text there are terms associated with the accelerator physics terminology. Definitions of these terms will be introduced briefly in this section. For more information about these terms, a very useful handbook for accelerator terminology is referred in Ref. [8]. Comprehensive coverage of non-linear beam dynamics and other special topics can be found in these references $[9,10,11,12]$.

\section{I.1.1 Equation of Motion}

As almost in all types of physical systems it is the first approach to find the most appropriate coordinate and reference systems. In accelerator physics, particle trajectories are tracked relative to an ideal path. Most of the time the ideal path is fixed ( $y$-axis in up direction) Cartesian reference system $(\mathbf{x}, \mathbf{y}, \mathbf{z})$. In the literature, this ideal path is also called nominal path, nominal trajectory or reference orbit. We are, most of the time, interested in the particle's trajectory in the proximity of this specific ideal path or reference orbit once the accelerator machine is set up properly. Then the particle's trajectory (orbit) can be defined with a right-handed orthogonal moving reference system $(\mathbf{x}, \mathbf{y}, \mathbf{s})[6]$. In Fig. 1, this reference system is shown with the particle's trajectory moving close the the ideal path. Most of the time the particle moves not on a straight line but this trajectory may have many complicated curves. This particle's trajectory can be defined with this curvilinear $\mathbf{r}(\mathbf{s})$ vector making it suitable for curved orbits in addition to the linear orbits.

Accelerator physics consists of many different types of fundamental physics, but dominant fundamental physics process is the electromagnetic physics. The electric (E) and magnetic (B) fields interact only with the charged particles via a force known as the Lorentz force:

$$
\frac{d \boldsymbol{p}}{d t}=\frac{d(\gamma m \boldsymbol{v})}{d t}=\mathbf{F}=e(\mathbf{E}+\boldsymbol{v} \times \mathbf{B})
$$

where $\boldsymbol{p}$ is the relativistic momentum vector of the particle; $\gamma, m, \boldsymbol{v}$ and $e$ are the relativistic Lorentz factor, the rest mass, the velocity and the unit charge of the particle respectively. If the force is acting on multi-charged particles, then the equation must be multiplied with the total charge number. 


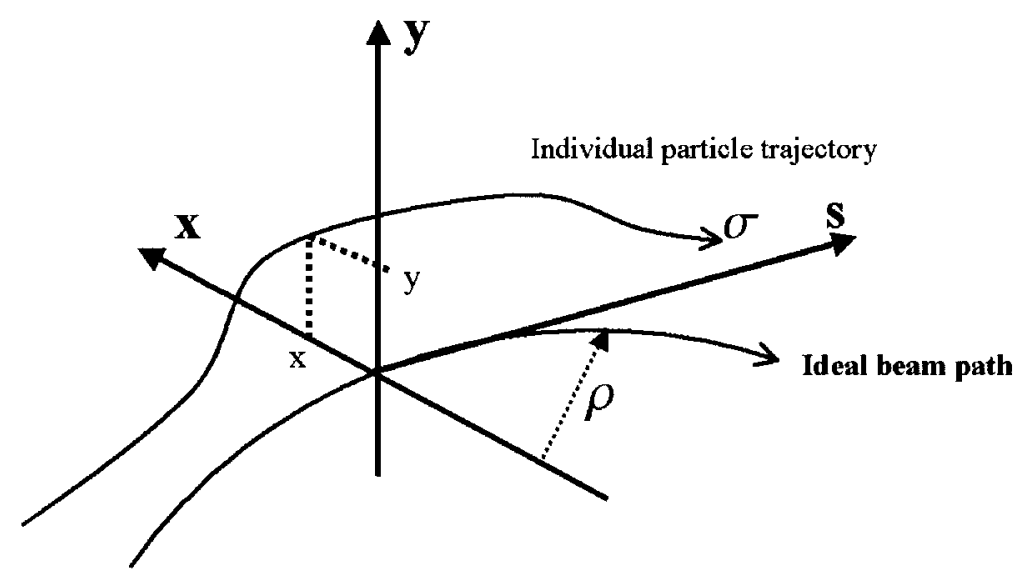

FIG. 1: The coordinate system showing the ideal trajectory and an individual particle trajectory in a moving generalized Cartesian coordinate system. Throughout this section this convention will be used, coordinate system is from Ref.[6].

Here the time derivative of the particle's momentum is given:

$$
\begin{aligned}
\frac{d \boldsymbol{p}}{d t} & =\gamma m \frac{d \boldsymbol{v}}{d t}+m \boldsymbol{v} \frac{d \gamma}{d t} \\
& =m\left(\gamma \frac{d \boldsymbol{v}}{d t}+\gamma^{3} \frac{\beta}{c} \frac{d v}{d t} \boldsymbol{v}\right)
\end{aligned}
$$

where the the Lorentz factor $(\gamma)$ and its time derivative, and the relativistic speed $(\beta)$ are given as:

$$
\begin{gathered}
\gamma=\frac{1}{\sqrt{1-\frac{v^{2}}{c^{2}}}}=\frac{1}{\sqrt{1-\beta^{2}}} \\
\frac{d \gamma}{d t}=\frac{d}{d \beta} \frac{1}{\sqrt{1-\beta^{2}}} \frac{d \beta}{d t}=\gamma^{3} \frac{\beta}{c} \frac{d v}{d t}
\end{gathered}
$$

By using these derivations, we can now rewrite Eq. (2) by separating parallel and normal components:

$$
\begin{aligned}
\frac{d \boldsymbol{p}_{\|}}{d t} & =m \gamma\left(1+\gamma^{2} \beta \frac{v}{c}\right) \frac{d \boldsymbol{v}_{\|}}{d t}=m \gamma^{3} \frac{d \boldsymbol{v}_{\|}}{d t} \\
\frac{d \boldsymbol{p}_{\perp}}{d t} & =m \gamma \frac{d \boldsymbol{v}_{\perp}}{d t}
\end{aligned}
$$


which we used $\dot{v} \boldsymbol{v}=\dot{\boldsymbol{v}} v$ relation when the force is parallel $\left(\frac{d \boldsymbol{p}_{\|}}{d t}\right)$ to the particle propagation, and when the force is directed normal $\left(\frac{d p_{\perp}}{d t}\right)$ to the propagation, then $\dot{v}=0$. As clearly seen, for highly relativistic particles the dynamics of the particles are greatly impacted with the direction of the Lorentz force.

We can now derive the transverse motion of the particle dynamics by making the assumptions of in the absence of the electric field $(\mathbf{E}=0)$ and by having only the transverse magnetic field $\mathbf{B}=\left(\mathrm{B}_{x}, \mathrm{~B}_{y}, 0\right)$ with a beam moving parallel to the $\mathbf{s}$ only with a velocity $\boldsymbol{v}=\left(0,0, v_{s}\right)$. With these assumptions, then the force is reduced to $\mathrm{F}_{x}=e v_{s} \mathrm{~B}_{y}\left(\mathrm{~F}_{y}=-e v_{s} \mathrm{~B}_{x}\right)$. Considering that the centrifugal force $\mathrm{F}_{r}=\gamma m v_{s}^{2} / \rho$ must be balanced with this force, which $\rho$ is the curvature of the trajectory as given in the reference coordinate system. From this balance we get the following:

$$
e v_{s} \mathrm{~B}=\frac{\gamma m v_{s}^{2}}{\rho}
$$

where the bending radius for the particle trajectory in a magnetic field $\mathbf{B}$ is defined:

$$
\frac{1}{\rho}=\left|\frac{e \mathbf{B}}{p}\right|=\left|\frac{e c}{\beta E} \mathbf{B}\right|
$$

Let us expand $y$ component of the magnetic field in Taylor series in the vicinity of the reference orbit since the transverse size of the beam is much smaller than the radius of curvature $x \ll \rho$, and then multiplying both sides by $e / \mathrm{p}$ gives us:

$$
\begin{aligned}
& \frac{e}{\mathrm{p}} \mathrm{B}_{y}(\mathrm{x})=\frac{e}{p} \mathrm{~B}_{y 0}+\frac{e}{p} \frac{\partial \mathrm{B}_{y}}{\partial x} x+\frac{e}{p} \frac{1}{2 !} \frac{\partial^{2} \mathrm{~B}_{y}}{\partial x^{2}} x^{2}+\frac{e}{p} \frac{1}{3 !} \frac{\partial^{3} \mathrm{~B}_{y}}{\partial x^{3}} x^{3}+\ldots \\
& =\frac{1}{\rho}+\mathrm{k} x+\frac{1}{2 !} \mathrm{m} x^{2} \quad+\frac{1}{3 !} \mathrm{o} x^{3} \quad+\ldots
\end{aligned}
$$

By expanding this magnetic field around the reference orbit we have obtained dipole, quadrupole, sextupole, octupole, etc. effects of the magnetic field on the particle. The first two terms of this expansion forms the linear optics, where third and higher order terms are studied in non-linear beam dynamics. Table 1 lists the definitions and functions of these multipole elements of the magnetic field.

From Eq. (7), it is possible to correlate a particle's momentum to the applied magnetic field, where we get a quantity called magnetic rigidity:

$$
|\mathbf{B} \rho|=\frac{p}{|e|}
$$

Magnetic rigidity is a convenient way to characterize the impact of the magnetic field as it only depends on the charge and momentum of the particle. After defining the 
TABLE 1: Description of the first few multipoles of the magnetic field $\mathbf{B}$ [5].

\begin{tabular}{lll}
\hline \hline Multipole & Element & Function \\
\hline Dipole & $\frac{1}{\rho}=\frac{e}{p} \mathrm{~B}_{y}$ & Beam steering \\
Quadrupole & $\mathrm{k}=\frac{e}{p} \frac{\partial \mathrm{B}_{y}}{\partial x}$ & Beam focusing \\
Sextupole & $\mathrm{m}=\frac{e}{p} \frac{\partial^{2} \mathrm{~B}_{y}}{\partial x^{2}}$ & Chromaticity compensation \\
Octupole & $\mathrm{o}=\frac{e}{p} \frac{\partial^{3} \mathrm{~B}_{y}}{\partial x^{3}}$ & Correcting field errors \\
\hline \hline
\end{tabular}

rigidity it is also convenient to take $\mathbf{s}$ as an independent variable and change from the time derivative. If we utilize ()$^{\prime}=d / d s$ and $(\dot{)}=d / d t$ in the differential equations, we can write the transformation from time derivative to $s$ :

$$
\begin{aligned}
x^{\prime} & =\frac{d x}{d s}=\frac{1}{\dot{s}} \dot{x} \\
x^{\prime \prime} & =\frac{d^{2} x}{d s^{2}}=\frac{1}{\dot{s}^{2}} \ddot{x}=-\frac{1}{\rho^{2}} x
\end{aligned}
$$

with the assumption $d \dot{s} / d s \approx 0$. Then the Lorentz force for a pure quadrupole field can be written as:

$$
\gamma m \frac{d v_{x}}{d s}=-e \frac{\partial \mathrm{B}_{y}}{\partial x} x
$$

From this we get the following in $x$-y directions with $v_{z} \approx \dot{s}$;

$$
\begin{array}{cc}
\frac{d^{2} x}{d s^{2}}+\frac{1}{\mathrm{~B} \rho} \frac{\partial \mathrm{B}_{y}}{\partial x} x=0 & \frac{d^{2} y}{d s^{2}}-\frac{1}{\mathrm{~B} \rho} \frac{\partial \mathrm{B}_{y}}{\partial x} y=0 \\
\frac{d^{2} x}{d s^{2}}=-\frac{x}{\rho_{x}^{2}(s)} & \frac{d^{2} y}{d s^{2}}=-\frac{y}{\rho_{y}^{2}(s)}
\end{array}
$$

As it is illustrated in Fig. 2, there will be off momentum particles which follow a different trajectory depending on their momentum. This relation is given by a function called Dispersion $(\eta)$ function, where it is related with the radius of curvature $1 / \rho$. 


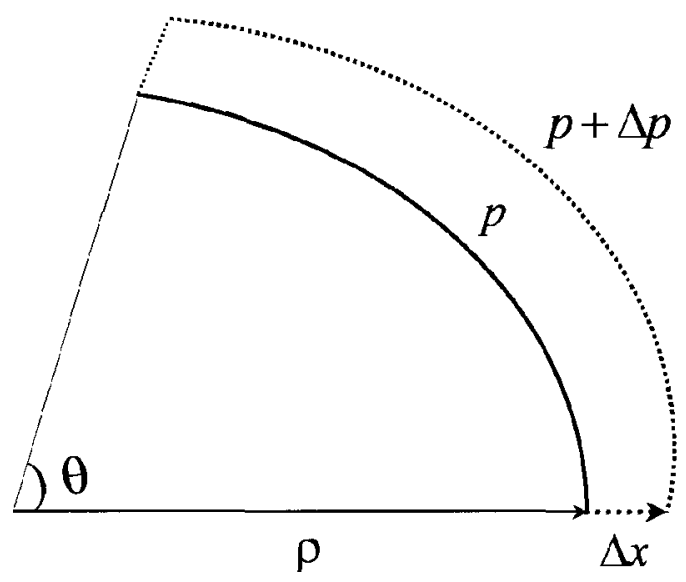

FIG. 2: The design momentum $p$ with with its momentum rigidity $\mathrm{B} \rho$ follows a design trajectory by $d s=\rho d \theta$. The beam is not always monochromatic, which has off-momentum particles. An off-momentum particle follows a perturbed trajectory, which is deviated from the design orbit by $\triangle x$.

If the amount of deviation from the central momentum is given by $\Delta p$, then we can find the amount of deviation from the central orbit with the dispersion:

$$
\begin{aligned}
p+\triangle p & =e \mathrm{~B}_{y}(\rho+\triangle x) \\
\triangle x & =\eta_{x} \delta
\end{aligned}
$$

where $\delta \equiv\left(p-p_{0}\right) / p_{0}=\Delta p / p_{0}$ and $\eta$ is the dispersion function with $x_{\delta}^{\prime \prime}=\eta_{x}^{\prime \prime} \delta$. The letter $D$ is also commonly used as a symbol to show the dispersion function. Combining Eq. (12) and Eq. (13) including the off-momentum part, we get:

$$
\begin{gathered}
\frac{d^{2} x}{d s^{2}}+\left[\frac{1}{\rho_{x}^{2}(s)}+\frac{1}{\mathrm{~B} \rho} \frac{\partial \mathrm{B}_{y}}{\partial x}\right] x=\frac{1}{\rho_{x}(s)} \delta \\
\frac{d^{2} y}{d s^{2}}+\left[\frac{1}{\rho_{y}^{2}(s)}-\frac{1}{\mathrm{~B} \rho} \frac{\partial \mathrm{B}_{y}}{\partial x}\right] y=\frac{1}{\rho_{y}(s)} \delta
\end{gathered}
$$

The relation can be simplified for on-momentum $(\delta=0)$ particle motion with:

$$
\frac{d^{2} x}{d s^{2}}+K_{x}(s) x=0 \quad \frac{d^{2} y}{d s^{2}}+K_{y}(s) y=0
$$

where $K_{x}$ and $K_{y}$ are the field strengths with:

$$
K_{x}=\left[\frac{1}{\rho_{x}^{2}(s)}+\frac{1}{\mathrm{~B} \rho} \frac{\partial \mathrm{B}_{y}}{\partial x}\right] \quad K_{y}=\left[\frac{1}{\rho_{y}^{2}(s)}-\frac{1}{\mathrm{~B} \rho} \frac{\partial \mathrm{B}_{y}}{\partial x}\right]
$$


These equations are known as Hill's equations. The $1 / \rho^{2}$ term comes from a pure dipole field which is known as a source of weak focusing; $\partial \mathrm{B}_{y} / \partial x$ term (the field gradient) comes from the pure quadrupole known as source of the strong focusing. This equation is familiar to us from the simple harmonic motion except the fact that $\mathrm{K}$ is not constant in Hill's equation. The solution of this inhomogeneous second order differential equation can be divided in two parts:

$$
x=x_{h}+x_{p}
$$

with $x_{h}$ being the homogeneous solution of the equation -when the right hand side of the Eq. (15) where $\delta=0$ - and here $x_{p}$ is the particular solution with the right hand side $\delta \neq 0$. The homogeneous side of the equation has a general solution:

$$
x_{h}=A \sin \left(\phi_{s}\right)+B \cos \left(\phi_{s}\right)
$$

or hyperbolic form of the sine and cosine functions depending on the sign of the equation. Since the Wronskian of the solutions of the homogenous equation has a determinant other than zero, these solutions are considered as linearly independent solutions. When there is only uniform dipole field term, then the equation has the solution:

$$
\begin{aligned}
x(s) & =\cos \left(\frac{s-s_{i}}{\rho}\right) x\left(s_{i}\right)+\rho \sin \left(\frac{s-s_{i}}{\rho}\right) x^{\prime}\left(s_{i}\right) \\
x^{\prime}(s) & =-\frac{1}{\rho} \sin \left(\frac{s-s_{i}}{\rho}\right) x\left(s_{i}\right)+\cos \left(\frac{s-s_{i}}{\rho}\right) x^{\prime}\left(s_{i}\right)
\end{aligned}
$$

When there is no dipole field but only quadrupole element, then the solution has the following form where for simplicity subscript of $\mathrm{K}$ is ignored:

$$
\begin{aligned}
& x(s)=\cos \left(\sqrt{K}\left(s-s_{i}\right)\right) x\left(s_{i}\right)+\frac{1}{\sqrt{K}} \sin \left(\sqrt{K}\left(s-s_{i}\right)\right) x^{\prime}\left(s_{i}\right) \\
& x^{\prime}(s)=-\sqrt{K} \sin \left(\sqrt{K}\left(s-s_{i}\right)\right) x\left(s_{i}\right)+\cos \left(\sqrt{K}\left(s-s_{i}\right)\right) x^{\prime}\left(s_{i}\right)
\end{aligned}
$$

where K includes only the quadrupole strength. A quadrupole's magnetic field allows focusing in one direction (say $x$ ) and defocusing in the other direction simultaneously. As a convention, when $\mathrm{K}>0$ it is focusing in the $x$ direction and defocusing in the other direction with hyperbolic trigonometric functions replacing the sine and cosine terms.

As a convenient way the equations given can also be written with a matrix formalism. The general formalism is that a transform matrix $\mathbf{M}$ carries a particle from 
a position $s_{i}$ to another position $s$ :

$$
\mathbf{X}(s)=\mathbf{M}_{s, s_{i}} \mathbf{X}\left(s_{i}\right)
$$

The dipole matrix transformation in Eq. (20) can be written as:

$$
\mathbf{X}(s)=\left(\begin{array}{c}
x(s) \\
x^{\prime}(s)
\end{array}\right)=\left(\begin{array}{rr}
\cos \left(\frac{s-s_{i}}{\rho}\right) & \rho \sin \left(\frac{s-s_{i}}{\rho}\right) \\
-\frac{1}{\rho} \sin \left(\frac{s-s_{i}}{\rho}\right) & \cos \left(\frac{s-s_{i}}{\rho}\right)
\end{array}\right)\left(\begin{array}{c}
x\left(s_{i}\right) \\
x^{\prime}\left(s_{i}\right)
\end{array}\right)
$$

Similarly the quadrupole transformation from Eq. (21) can be written in the matrix formalism as:

$$
\left(\begin{array}{c}
x(s) \\
x^{\prime}(s)
\end{array}\right)=\left(\begin{array}{cc}
\cos \left(\sqrt{K}\left(s-s_{i}\right)\right) & \frac{1}{\sqrt{K}} \sin \left(\sqrt{K}\left(s-s_{i}\right)\right) \\
-\sqrt{K} \sin \left(\sqrt{K}\left(s-s_{i}\right)\right) & \cos \left(\sqrt{K}\left(s-s_{i}\right)\right)
\end{array}\right)\left(\begin{array}{c}
x\left(s_{i}\right) \\
x^{\prime}\left(s_{i}\right)
\end{array}\right)
$$

Then the full transverse matrix for a quadrupole of length $L$ has the following form:

$$
\begin{aligned}
\left(\begin{array}{c}
x(s) \\
x^{\prime}(s) \\
y(s) \\
y^{\prime}(s)
\end{array}\right) & =\left(\begin{array}{cccc}
\cos (\sqrt{K} L) & \frac{1}{\sqrt{K}} \sin (\sqrt{K} L) & 0 & 0 \\
-\sqrt{K} \sin (\sqrt{K} L) & \cos (\sqrt{K} L) & 0 & 0 \\
0 & 0 & \cosh (\sqrt{K} L) & \frac{1}{\sqrt{K}} \sinh (\sqrt{K} L) \\
0 & 0 & -\sqrt{K} \sinh (\sqrt{K} L) & \cosh (\sqrt{K} L)
\end{array}\right) \\
& \times\left(\begin{array}{c}
x\left(s_{i}\right) \\
x^{\prime}\left(s_{i}\right) \\
y\left(s_{i}\right) \\
y^{\prime}\left(s_{i}\right)
\end{array}\right)
\end{aligned}
$$

When the dipole and quadrupole terms are equal to zero, then the particle moves without influence of the electromagnetic fields, this is called drift transformation (drift space), which the matrix form is:

$$
\left(\begin{array}{c}
x(s) \\
x^{\prime}(s)
\end{array}\right)=\left(\begin{array}{cc}
1 & s-s_{i} \\
0 & 1
\end{array}\right)\left(\begin{array}{c}
x\left(s_{i}\right) \\
x^{\prime}\left(s_{i}\right)
\end{array}\right)
$$

\section{I.1.2 Thin lens approximation}

As it is seen from the transport matrix formalism of the equation of motion, it involves a great deal of matrix calculation with trigonometric functions. Each element in an accelerator machine is formulated by a matrix where dimensions could be as large as needed for higher order terms. Generally computer softwares are used to calculate 
these equations. As the particle beam is reminiscent of a light beam, we can see a similar connection in the infinitely thin lens approximation for light. Here we can use thin quadrupoles and simplify the matrix equations. As an example we can use a thick quadrupole transformation matrix given in Eq. (24). If the length of the quadrupole is taken to be $L$ and the strength $K$ then the focal length is defined as:

$$
f=\frac{1}{K L}
$$

In this thin lens approximation the length of the quadrupole is assumed to be very small compared to the focal length with $L \ll f$, where $L \rightarrow 0$. By using this approximation, then we can expand the solutions as: $\sin (\sqrt{K} L) \approx \sqrt{K} L=0$ and $\cos (\sqrt{K} L) \approx 1$. By replacing these in Eq. (24), we get the following:

$$
\left(\begin{array}{c}
x(s) \\
x^{\prime}(s)
\end{array}\right)=\left(\begin{array}{rr}
1 & 0 \\
-\frac{1}{f} & 1
\end{array}\right)\left(\begin{array}{c}
x\left(s_{i}\right) \\
x^{\prime}\left(s_{i}\right)
\end{array}\right)
$$

for the focusing part, and for the defocusing part the approximation has the similar result except the sign of the $1 / f$ turns to positive.

\section{I.1.3 Phase ellipse and transverse emittance}

In a generalized canonical coordinate system $\left(q_{i}, p_{j}\right)$ where $q_{i}$ and $p_{j}$ are generalized position and conjugate momentum of the particles respectively, the phase space area filled in these coordinates is conserved as long as it is under linear conservative forces according to the Liouville's theorem. A single particle in this conserved phase space, can be located by its transverse positions and their conjugate phase space coordinates $\left(x, p_{x} ; y, p_{y}\right)$. If $x-y$ coordinates are coupled, then the $x \otimes p_{x}$ and $y \otimes p_{y}$ areas are not separately conserved. In general, we deal with many particles and we are only interested in their collective behaviour with their boundary conditions. These particles form a beam if they are in a small and condensed area and deviate only by small amounts from the central trajectory. Most of the time we are also interested in the particles slope $d x / d s \equiv p_{x} / p=x^{\prime}$, not in their transverse momentum $p_{x}$, where the canonical momentum is replaced with $x^{\prime}$.

By using this $x-x^{\prime}$ phase space, the beam can usually be introduced by a normalized distribution or density function $f\left(x, x^{\prime}\right)$, where the integration over this phase space gives the unity:

$$
\iint f\left(x, x^{\prime}\right) d x d x^{\prime}=1
$$




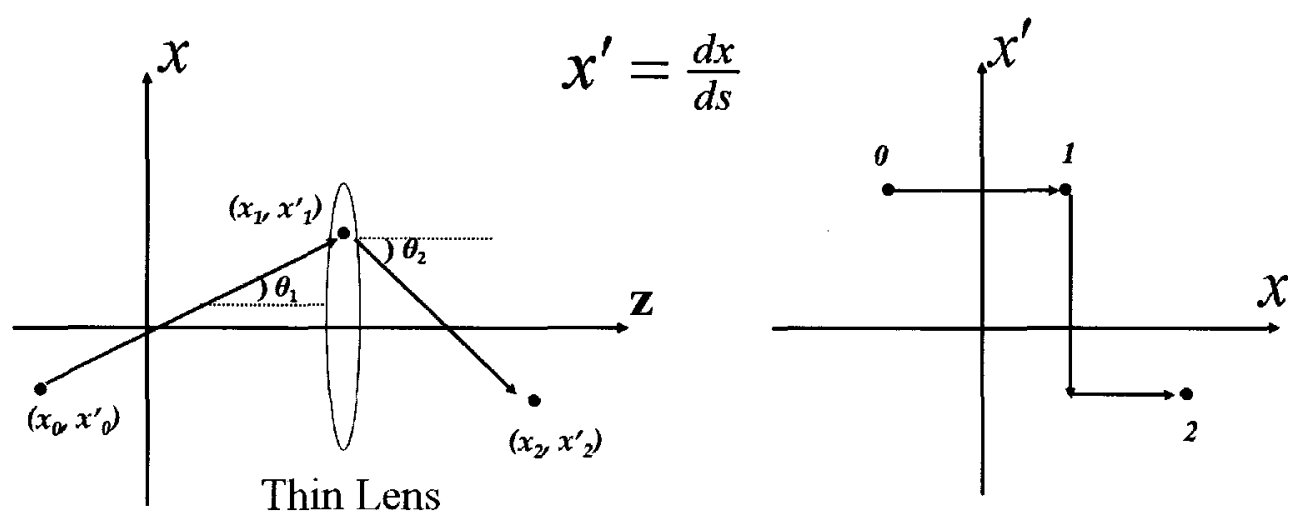

FIG. 3: On the left, a particle's movement is shown in the cartesian coordinate where $x^{\prime}=d x / d s$ is the slope of the particle which it makes in the horizontal plane. Here the particle has $x_{0}$ as the initial position traveling in a drift space with an angle $\theta_{1}$, where at $x_{1}$ a thin quadrupole changes the angle to $-\theta_{2}$. The particle's movement is illustrated in $x-x^{\prime}$ phase space on the right coordinate system.

Most of the time the beam distribution is type of a multivariate gaussian distribution, which the beam is parameterized by using its average and $1 \sigma$ parameters.

As it is seen in Fig. 3, the behaviour of a single particle is illustrated when traveling in a drift space and followed by a thin focusing lens in cartesian coordinate. The particle's movement around the reference orbit is also shown in the same figure within $x-x^{\prime}$ phase space coordinate (a.k.a trace-space coordinate). From the figure, it is shown that regardless of particle's position along the longitudinal axis, on $x-x^{\prime}$ coordinate system we only get information about its $x$ and $x^{\prime}$. Of course the beam is not formed of a single particle but many of them with different $x$ and $x^{\prime}$ s. When thousands of particles make small random deviations from the reference orbit, they form a phase space area, which this area can be formulated by integrating the density function over the coordinates as:

$$
\begin{aligned}
<x> & =\iint x f\left(x, x^{\prime}\right) d x d x^{\prime} \\
<x^{\prime}> & =\iint x^{\prime} f\left(x, x^{\prime}\right) d x d x^{\prime} \\
\sigma_{x}^{2} & =\iint(x-<x>)^{2} f\left(x, x^{\prime}\right) d x d x^{\prime} \\
\sigma_{x^{\prime}}^{2} & =\iint\left(x^{\prime}-<x^{\prime}>\right)^{2} f\left(x, x^{\prime}\right) d x d x^{\prime}
\end{aligned}
$$


where $\langle x\rangle$ and $\sigma_{x}$ are average position and r.m.s deviation from this position; $\left\langle x^{\prime}\right\rangle$ and $\sigma_{x^{\prime}}$ average slope and r.m.s deviation from the slope in $x$ coordinate in respectively. If there is a correlation between $x$ and $x^{\prime}$, then the correlation is:

$$
\begin{aligned}
\sigma_{x x^{\prime}} & =\iint(x-<x>)\left(x^{\prime}-<x^{\prime}>\right) f\left(x, x^{\prime}\right) d x d x^{\prime} \\
& =r \sigma_{x} \sigma_{x}^{\prime}
\end{aligned}
$$

where $r$ is the correlation coefficient. We can now define the phase space area, emittance $(\varepsilon)$ of the beam with:

$$
\begin{aligned}
& \varepsilon_{x}^{2}=\sigma_{x}^{2} \sigma_{x^{\prime}}^{2}-\sigma_{x x^{\prime}}^{2} \\
& \varepsilon_{x}=\sigma_{x} \sigma_{x^{\prime}} \sqrt{1-r^{2}}
\end{aligned}
$$

if the phase space area is upright, with correlation $r=0$, then the emittance is simply $\varepsilon_{x}=\sigma_{x} \sigma_{x^{\prime}}$. The phase space area has usually the shape of an ellipse where the area $(A)$ is the production of the semi-major axes $a$ and $b$ :

$$
A=\pi a \cdot b
$$

In a beam distribution with tilted ellipse, the area and beam emittance are related with:

$$
A=\pi \varepsilon_{x}=\pi \sigma_{x} \sigma_{x^{\prime}} \sqrt{1-r^{2}}
$$

The emittance, or to say more correctly in this context, transverse geometrical emittance has different definitions which often lead to confusion. But in fact it is not because emittance lacks a definition, it is because the area of the phase ellipse is sometimes used as emittance, or sometimes only the semi-axes multiplication is quoted as the emittance. It is also common to quote 1- $\sigma$ (or r.m.s) emittance as the emittance if the beam distribution is gaussian type. There is also a usage of $\pi$ in the units which intends to factor out double multiplication of the emittance to get the area. To solve this confusion a good practice would require to state whether the emittance is an area emittance or other type of emittance. Throughout the text I always quote 1- $\sigma$ (or rms) geometrical emittance unless otherwise noted.

In experiments or simulations, where statistical processes dominate the particle behaviour; the emittance of the beam can be introduced with statistical averages of the transverse position and angles of the beam as well: 


$$
\begin{aligned}
\varepsilon_{x} & =\sqrt{\left\langle x^{2}\right\rangle\left\langle x^{\prime 2}>-<x x^{\prime}\right\rangle^{2}} \\
& =\sqrt{\frac{1}{N} \sum_{i=1}^{N}\left(x_{i}-\bar{x}\right)^{2} \frac{1}{N} \sum_{i=1}^{N}\left(x_{i}^{\prime}-\bar{x}^{\prime}\right)^{2}-\left(\frac{1}{N} \sum_{i=1}^{N}\left(x_{i}-\bar{x}\right)\left(x_{i}^{\prime}-\bar{x}^{\prime}\right)\right)^{2}}
\end{aligned}
$$

In accelerator physics, most often these averages are not quoted but there is a set of

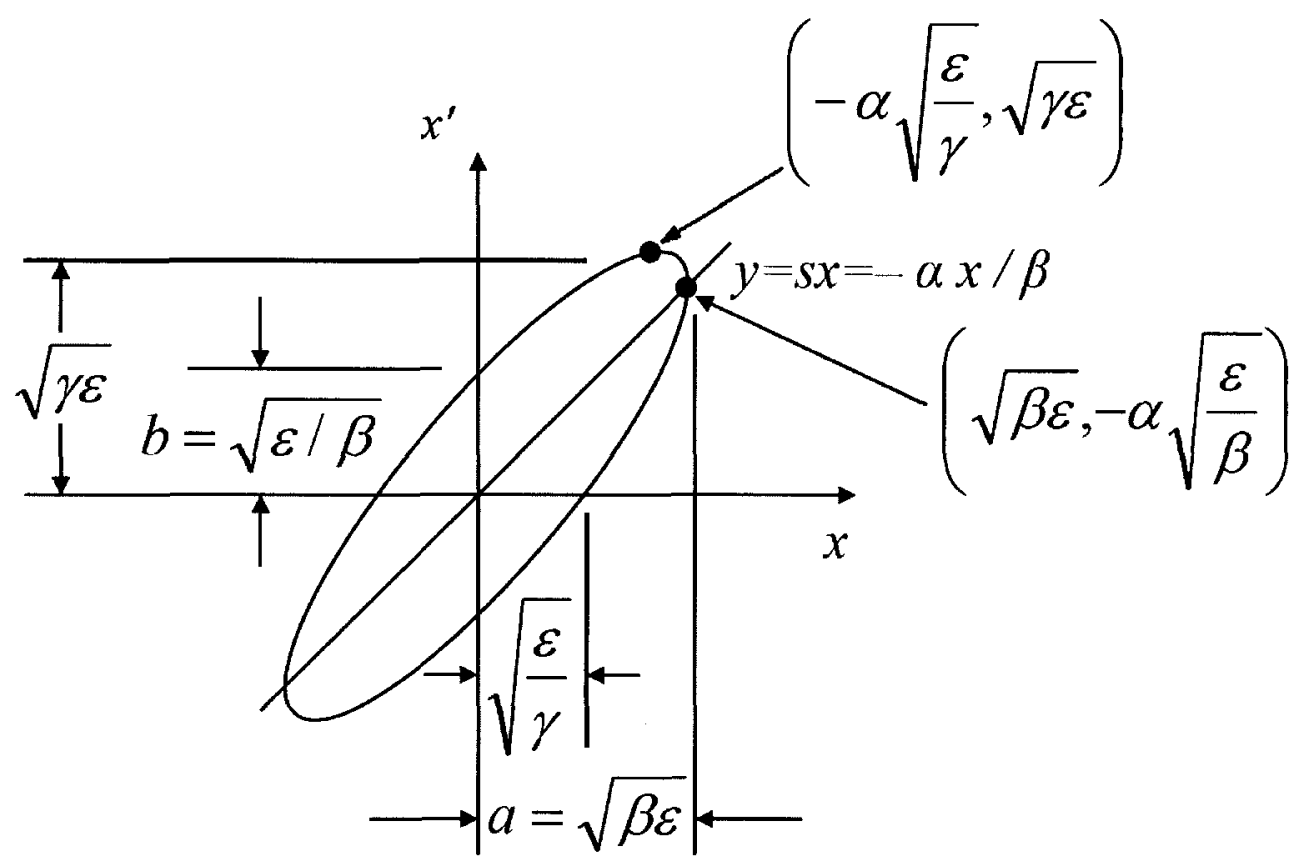

FIG. 4: The phase space ellipse of the beam representing the area that it occupies is plotted [5]. Here $\beta, \alpha$ and $\gamma$ are twiss parameters of the beam. The $\varepsilon$ is the emittance of the beam with $\varepsilon=$ Area $/ \pi$.

terms called Courant-Snyder parameters [13] (aka Twiss parameters) to define the phase ellipse. A typical phase ellipse and its features are shown in Fig. 4. In this tilted ellipse the Courant-Snyder invariant emittance is defined as:

$$
\gamma x^{2}+2 \alpha x x^{\prime}+\beta x^{2}=\varepsilon_{x}
$$


where $(\alpha, \beta, \gamma)$ are the Courant-Snyder parameters (not to be confused with relativistic $\beta$ and $\gamma$ ) defined as:

$$
\begin{aligned}
& \beta=\frac{<x^{2}>}{\varepsilon_{x}} \\
& \gamma=\frac{<x^{\prime 2}>}{\varepsilon_{x}} \\
& \alpha=-\frac{<x x^{\prime}>}{\varepsilon_{x}}
\end{aligned}
$$

The Courant-Snyder variables; $\alpha, \beta$ and $\gamma$ define co-centric ellipses with the same ratio of semi-axes. A particle in an ensemble with a transverse position and angle $\left(x, x^{\prime} ; y, y^{\prime}\right)$ will be a member of this ellipse if it satisfies the relation given in Eq. (36). The beam r.m.s transverse size $\sigma_{x, y}(s)$ is correlated with the beam emittance with the following relation:

$$
\sigma(s)=\sqrt{\varepsilon \beta(s)}
$$

and the $\alpha$, which is the correlation coefficient, given as the negative derivative of the $\beta$ function with:

$$
\alpha=-\frac{1}{2} \frac{d \beta_{x}}{d s}
$$

There is also a relation between $\gamma$ and $\alpha$, which is defined as:

$$
\gamma \equiv \frac{\alpha^{2}+1}{\beta}
$$

By using Courant-Snyder variables, we can easily track the beam evolution which can be described by defining a $\beta$ matrix (B) (sometimes used as $\Sigma$ matrix when the $\varepsilon$ is factored out) as:

$$
\mathbf{B}_{0} \equiv\left(\begin{array}{cc}
\beta_{0} & -\alpha_{0} \\
-\alpha_{0} & \gamma_{0}
\end{array}\right)
$$

with $\operatorname{det}\left(\mathbf{B}_{0}\right)=1$. If we use the trajectory vector, which was defined in Eq. (22), with the transpose vector $\mathbf{X}_{\mathbf{0}}^{\mathbf{T}}=\left(x_{0} x_{0}^{\prime}\right)$; then the matrix product gives:

$$
\begin{aligned}
\mathbf{X}_{\mathbf{0}}^{\mathbf{T}} \cdot \mathbf{B}_{0}^{-1} \cdot \mathbf{X}_{\mathbf{0}} & =\left(\begin{array}{ll}
x_{0} & x_{0}^{\prime}
\end{array}\right) \cdot\left(\begin{array}{cc}
\beta_{0} & -\alpha_{0} \\
-\alpha_{0} & \gamma_{0}
\end{array}\right) \cdot\left(\begin{array}{l}
x_{0} \\
x_{0}^{\prime}
\end{array}\right) \\
& =\gamma_{0} x_{0}^{2}+2 \alpha_{0} x_{0} x_{0}^{\prime}+\beta_{0} x_{0}^{\prime}
\end{aligned}
$$

For a gaussian beam distribution, the $\left(x-x^{\prime}\right)$ probability distribution $P\left(\mathbf{X}_{\mathbf{0}}\right)$ is:

$$
P\left(\mathbf{X}_{\mathbf{0}}\right)=\frac{1}{2 \pi \varepsilon} \exp \left(-\frac{\mathbf{X}_{\mathbf{0}}^{\mathbf{T}} \cdot \mathbf{B}_{0}^{-1} \cdot \mathbf{X}_{\mathbf{0}}}{2 \varepsilon}\right)
$$


This beta matrix gives us a very convenient way to calculate the particles final position $\mathbf{X}_{f}(\mathrm{~s})$ if the initial position $\mathbf{X}_{0}$ and the transformation matrix are given. The final beta matrix $\mathbf{B}_{f}$ has the following equation:

$$
\mathbf{B}_{f}=\mathbf{M}_{s_{0}, s_{f}} \cdot \mathbf{B}_{0} \cdot \mathbf{M}_{s_{0}, s_{f}}^{\mathbf{T}}
$$

It is worth to note here that the $\mathbf{M}$ transformation matrix can be the result of several matrices where each matrix represents the transformation matrix of its own kind such as a quadrupole given in Eq. (24). The total matrix is the multiplication of all elements in the machine including the drift space and magnets. The next element is multiplied from the left side of the prior, giving us:

$$
\mathbf{M}_{s_{0}, s_{f}}=\mathbf{M}_{f} \ldots . . \mathbf{M}_{2} \mathbf{M}_{1} \mathbf{M}_{0}
$$

The twiss parameters are very useful tools to understand how the beam behaves when going under a series of transformations. Although the geometrical emittance is an invariant of the motion (in the absence of acceleration), the shape of the ellipse changes from point to point. The phase space evolution of the beam is illustrated in Fig. 5. Here we see a set of quadrupoles where F (D) is focusing (defocusing) in $x$. In this figure it is seen that, at the narrowest $\beta$ function, the phase space is in upright position with $\alpha=0$, this is also the position where the beam has the smallest size in the figure. The $\beta$ function starts diverging and finally reaches the climax again. 


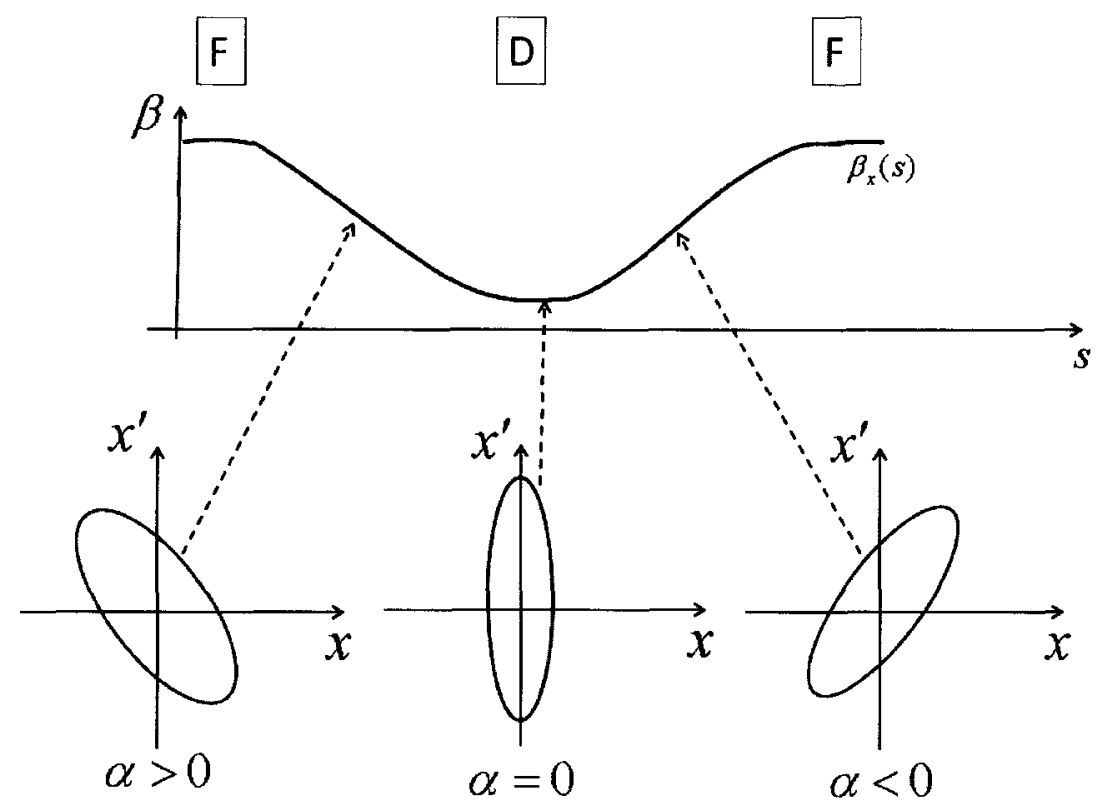

FIG. 5: Transformation of the $x-x^{\prime}$ phase space ellipse is shown in a periodic focusing $(\mathrm{F})$-defocusing (D) quadrupole channel. The location where the $\beta_{x}(s)$ has the minimum value and maximum value is called the waist position. In the left, the beam is focused to a narrow waist $(\alpha>0)$; in the middle the beam has the minimum $\beta$ value $(\alpha=0)$; and in the right the beam is diverging $(\alpha<0)$.

\section{I.1.4 Dispersion Function}

The general solution for the inhomogeneous Hill's equation is described with Eq. (18) where the solution is divided into two parts; $x_{h}$ for the homogenous part with $\delta=0$ and $x_{p}$ particular solution for the inhomogeneous part with $\delta \neq 0$. As described in Eq. (14), this inhomogeneous part is related to the dispersion function where offmomentum particles do not follow the design orbit. The particular solution for this dispersion part is derived in a similar way to the homogenous part. Usually the $x_{h}$ is called the betatron motion shown with $x_{\beta}$. If we add the dispersion offset (in the bending plane) to the particle orbit, then the particle has the orbit function with:

$$
x(s)=x_{\beta}+\eta_{x} \frac{\Delta \mathrm{p}}{\mathrm{p}} \quad y(s)=y_{\beta}+\eta_{y} \frac{\Delta \mathrm{p}}{\mathrm{p}}
$$

where both the $x_{\beta}$ and the dispersion $\eta$ functions must satisfy the Hills's equation. And if there is a dispersion in $y$ (due to the misalignments etc.) it must also be 
considered. Then the equations of motion for the dispersion function becomes:

$$
\frac{d^{2} \eta_{x}}{d s^{2}}+K_{x}(s) \eta=\frac{1}{\rho(s)} \quad \frac{d^{2} \eta_{y}}{d s^{2}}+K_{y}(s) y=\frac{1}{\rho(s)}
$$

The solution for the homogenous part is the same as before which is a combination of two linearly independent functions, sine and cosine where $K$ includes both the dipole and quadrupole terms. The particular solution can be formalized with the following particular solution:

$$
\eta(s)=C_{n} \eta^{\mathrm{n}}+C_{n-1} \eta^{\mathrm{n}-1}+\ldots C_{0}
$$

where here $n$ is the degree of order and $C$ s are constant coefficients. When the right hand side of the Eq. (47) is a constant only, where all $C_{n}=C_{n-1}=. .=C_{1}=0$ giving us a particular solution $\eta=C_{0}$ and $\eta^{\prime \prime}=0$. Inserting this in the Eq. (47), we get the particular solution:

$$
\begin{aligned}
\eta^{\prime \prime}+K \eta & =\frac{1}{\rho} \\
K C_{0} & =\frac{1}{\rho} \\
C_{0} & =\frac{1}{K \rho}
\end{aligned}
$$

Combining the general solution of the homogenous equation and the particular solution, we get the full solution (which is also Combined Function Magnet solution when both dipole and quadrupole fields exist) to the dispersion function for a uniform quadrupole and dipole magnet:

$$
\eta(s)=A \sin (\sqrt{K} s)+B \cos (\sqrt{K} s)+\frac{1}{K \rho}
$$

The coefficients can be easily found by the initial conditions where at the beginning of the magnet at $s=0$ the dispersion and the derivative of the dispersion have the initial values $\eta(0)=\eta_{0}$ and $\eta^{\prime}(0)=\eta_{0}^{\prime}$. By using these initial values we get:

$$
\begin{aligned}
\eta(s) & =\frac{1}{K \rho}+\left[\eta(0)-\frac{1}{K \rho}\right] \cos (\sqrt{K} s)+\frac{\eta^{\prime}(0)}{\sqrt{K}} \sin (\sqrt{K} s) \\
\eta^{\prime}(s) & =\left[\frac{1}{\sqrt{K} \rho}-\sqrt{K} \eta(0)\right] \sin (\sqrt{K} s)+\eta^{\prime}(0) \cos (\sqrt{K} s)
\end{aligned}
$$

In the same way as we did before, we can rewrite the dispersion equation in a matrix formalism. Now we have the extra term as the dispersion, which we need to write it 
in $3 \times 3$ matrix:

$$
\left(\begin{array}{c}
\eta(s) \\
\eta^{\prime}(s) \\
1
\end{array}\right)=\left(\begin{array}{ccc}
\cos (\sqrt{K} L) & \frac{1}{\sqrt{K}} \sin (\sqrt{K} L) & \frac{1-\cos (\sqrt{K} L)}{K \rho} \\
-\sqrt{K} \sin (\sqrt{K} L) & \cos (\sqrt{K} L) & \frac{\sin (\sqrt{K} L)}{\sqrt{K} \rho} \\
0 & 0 & 1
\end{array}\right) \times\left(\begin{array}{c}
\eta(0) \\
\eta^{\prime}(0) \\
1
\end{array}\right)
$$

where $L$ is the magnet length with $L=s-s_{0}$. For defocusing systems where $K<0$, cosine and sine terms are replaced with hyperbolic equivalents as before.

Finally we can include our matrix with the betatron function:

$$
\left(\begin{array}{c}
x(s) \\
x^{\prime}(s) \\
y(s) \\
y^{\prime}(s) \\
\delta
\end{array}\right)=\mathbf{M} \cdot\left(\begin{array}{c}
x\left(s_{0}\right) \\
x^{\prime}\left(s_{0}\right) \\
y\left(s_{0}\right) \\
y^{\prime}\left(s_{0}\right) \\
\delta
\end{array}\right)
$$

where $\mathbf{M}$ matrix is the transformation matrix given in Eq. (52) with the hyperbolic terms included. If the field gradient $\mathrm{B}^{\prime}$ in the $\mathrm{K}$ is zero, then the equation must be considered as a pure dipole and the coefficients are recalculated accordingly. Since the dispersion is added as an offset to the beam size, this quantity must be added in Eq. (38). Then the r.m.s beam size including dispersion is given with:

$$
\sigma=\sqrt{\varepsilon \beta+(\eta \delta \mathrm{p})^{2}}
$$

\section{I.1.5 Achromat}

The dispersion comes as a natural side effect from the bending magnets. Although dispersion may be used to select energy in a fine tune way, if not matched properly or suppressed, it will cause the off-momentum particles lost by hitting the apertures in the machine. A set of magnets which controls the dispersion and makes the lattice independent from the momentum offsets is called an achromatic lattice. There are various lattice types to suppress the dispersion such as; double bend achromat (DBA) also called as Chasman-Green [14] lattice, triple bend achromat (TBA), triple achromat lattice (TAL), etc. I will discuss the DBA lattice type and use it as an example in the next chapters.

A double bend achromat consists of two bending magnets (dipoles) with a single quadrupole positioned at a symmetry point, which is in the middle of the lattice. 
The quadrupole features a focusing effect in the bending plane. The pre- and postachromat lattice must also be arranged so that the $\beta$ functions stay in a reasonably matched condition.

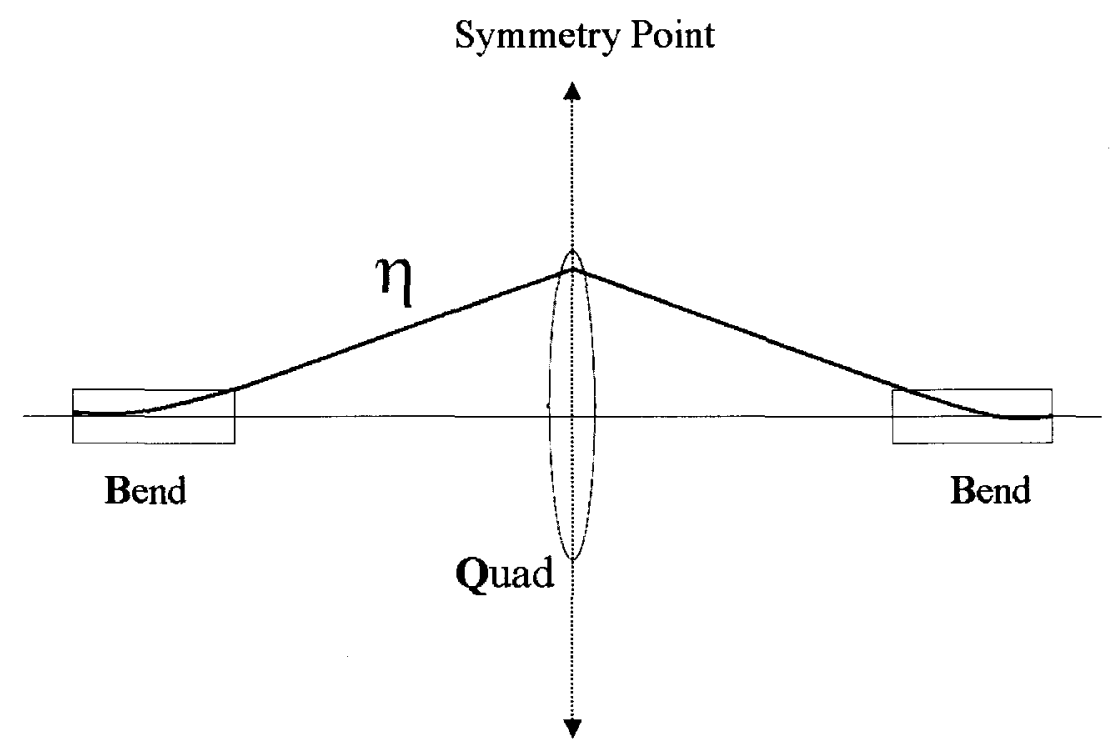

FIG. 6: The dispersion function $\eta$ in a Double Bend Achromat (DBA) lattice. There are two sector bending magnets with zero field gradient and a single focusing (in the bending plane) quadrupole located at the symmetry point of the lattice.

When an off-momentum particle enters the dipole, it starts to follow a different trajectory from the design orbit. This offset is related to its $\delta$ as it was given in Eq. (14). The basic idea behind the DBA is that the dispersion function must be suppressed by use of a quadrupole, which is focusing in the same direction of the bending plane. This idea is illustrated in Fig. 6. As it is seen from the figure the bends kick the dispersion in the opposite direction from the quadrupole. The key condition in the DBA is that we have a condition where $\eta$ must have a finite value and derivative of $\eta$ must be equal to $\eta^{\prime}=0$ at the symmetry point. The symmetry point is the center of the quadrupole magnet. The complexity of the matrix elements most often times requires computer programs to solve the equations, but here we can show a rough estimation by using thin lens approximation. We start of by dividing the lattice into two pieces from the symmetry point and use the half lattice on the left. This will give us a lattice in the following format:

$$
\left\{\text { Bend, Drift, } \frac{1}{2} \text { Quad }\right\}
$$


where the quadrupole is separated equally at the symmetry point.

In thin lens approximation the matrix elements for a sector dipole $\left(M_{B}\right)$, focusing half quadrupole $\left(\mathrm{M}_{\frac{1}{2} Q F}\right)$ and drift space $\left(\mathrm{M}_{D}\right)$ are given:

$$
\begin{aligned}
\mathrm{M}_{B} & =\left(\begin{array}{ccc}
1 & L & \frac{L \theta}{2} \\
0 & 1 & \theta \\
0 & 0 & 1
\end{array}\right) \\
\mathrm{M}_{\frac{1}{2} Q F} & =\left(\begin{array}{ccc}
1 & 0 & 0 \\
\frac{-1}{2 f} & 1 & 0 \\
0 & 0 & 1
\end{array}\right) \\
\mathrm{M}_{D} & =\left(\begin{array}{ccc}
1 & L_{d} & 0 \\
0 & 1 & 0 \\
0 & 0 & 1
\end{array}\right)
\end{aligned}
$$

where $L$ is the bending dipole length, $\theta$ is the bending angle, $f$ is the focal length of the quadrupole and $L_{d}$ is the drift length. As it is seen from Fig. 6, initially the dispersion is zero, which at the symmetry point it reaches the maximum point. So we can use the initial values for dispersion and its first derivative as $\eta(0)=\eta^{\prime}(0)=0$ and the values at the symmetry point are $\eta(s)=\eta_{s}$ and $\eta^{\prime}(s)=0$. By using these conditions we can write the equation of dispersive motion. The total transformation matrix composed of these elements is the product of the matrix elements in Eq. (56). Having the product and by applying general matrix transformation from $s_{0}$ to $s$ given in Eq. (22) we get:

$$
\begin{aligned}
\left(\begin{array}{c}
\eta(s) \\
\eta^{\prime}(s) \\
1
\end{array}\right) & =\mathrm{M}_{\frac{1}{2} Q F} \cdot \mathrm{M}_{D} \cdot \mathrm{M}_{B} \cdot\left(\begin{array}{c}
\eta(0) \\
\eta^{\prime}(0) \\
1
\end{array}\right) \\
\left(\begin{array}{c}
\eta_{s} \\
0 \\
1
\end{array}\right) & =\left(\begin{array}{ccc}
1 & 0 & 0 \\
\frac{-1}{2 f} & 1 & 0 \\
0 & 0 & 1
\end{array}\right)\left(\begin{array}{ccc}
1 & L_{d} & 0 \\
0 & 1 & 0 \\
0 & 0 & 1
\end{array}\right)\left(\begin{array}{ccc}
1 & L & \frac{L \theta}{2} \\
0 & 1 & \theta \\
0 & 0 & 1
\end{array}\right)\left(\begin{array}{l}
0 \\
0 \\
1
\end{array}\right) \\
& =\left(\begin{array}{c}
\frac{L \theta}{2}+L_{d} \theta \\
\theta\left(\frac{4 f-2 L_{d}-l}{4 f}\right) \\
1
\end{array}\right)
\end{aligned}
$$


Here we calculated the required focal length for a dispersion free lattice, where the focal length and resulting $\eta$ at the symmetry point will be:

$$
\begin{aligned}
f & =\frac{L_{d}}{2}+\frac{L}{4} \\
\eta_{s} & =\theta\left(\frac{L}{2}+L_{d}\right)
\end{aligned}
$$

\section{I.2 DEFINITIONS}

\section{Lattice}

The collection of the bending magnets, quadrupoles and other magnetic elements are called the magnet lattice (lattice).

\section{Beam current and time structure}

In accelerators, the time structure of the beam is important. It is vital to achieve a good quality timing where it helps to minimize unwanted effects such as acceleration with a fraction of the beam not getting the full gradient. We can classify the beam time structure into two groups; pulsed beam and continuous beam. In a beam the smallest unit is the microbunch, where microbunches form a macrobunch. Macrobunches form a train separated by a drift space which this type of beam structure is called pulsed beam. The pulsed beam is on and off in certain time periods. On the other type, the continuous beam is defined as the beam is always on. The current, by definition, is the amount of total electric charge $(Q)$ passing per unit time:

$$
I(\text { Ampere })=\frac{Q(\text { Coulomb })}{\tau(\mathrm{s})}
$$

In Fig. 7, the time structures for micro and macro bunch are illustrated. The microbunch current (peak current) in Fig. 7(a) has a charge of $q$ and distributed in time over $\tau_{\mu}$, the peak current $I$ for a square box distribution is:

$$
I=\frac{\text { Microbunch charge }}{\text { Microbunch duration }}=\frac{q}{\tau_{\mu}}
$$

In general, it should be emphasized whether the $\tau_{\mu}$ is a gaussian distribution or not. If the distribution is gaussian, then the time spread of $\tau_{\mu}$ is the $1 \sigma$ spread size of the microbunch, where the peak current has a factor of: 


$$
I=\frac{q}{\sqrt{2 \pi} \sigma_{\tau_{\mu}}} \exp \left(-\frac{x^{2}}{2 \sigma_{\tau_{\mu}}^{2}}\right)_{x=0}=\frac{q}{\sqrt{2 \pi} \sigma_{\tau_{\mu}}}
$$

In Fig. 7(b) the pulse current structure is shown where $I_{p}$ is the pulsed current defined as the average current during the duration of the pulse:

$$
I_{p}=\hat{I} \frac{\tau_{\mu}}{T_{\mu}}=\frac{q}{\tau_{\mu}}
$$

where $T_{\mu}$ is the time between successive microbunches. The microbunch structure is usually the same as the structure of the RF field where microbunches are separated by the wavelength of the RF. In Fig. $7(\mathrm{c})$, the average current $(\langle I\rangle)$ is explained. Microbunches form a pulse (macrobunch), where these pulses are separated by a certain time called the repetition time $\mathrm{T}_{r e p}$ or pulse repetition rate $\nu_{r e p}=\frac{1}{\mathrm{~T}_{\text {rep }}}$. The average current is then:

$$
<I>=I_{p} \frac{\mathrm{T}_{p}}{\mathrm{~T}_{r e p}}=\frac{\mathrm{n}_{\mu} q}{\mathrm{~T}_{r e p}}
$$

where $\mathrm{T}_{p}$ is the duration of the pulse and $\mathrm{n}_{\mu}$ is the total number of microbunches per pulse.

In the last graph the current line is constant where this type of beam is called continuous beam. In this configuration the beam is always on as opposed to the pulsed configuration. The beam on - beam off time configuration is an important parameter in the accelerator, especially in the RF. This parameter is measured by a term called the duty factor. The duty factor is the fraction of the active beam time to the total time in the accelerator. For example if the pulse length $T_{p}=1 \mathrm{~ms}$ and the pulse repetition period $\mathrm{T}_{r e p}=50 \mathrm{~ms}$, then the duty factor is:

$$
D F=\frac{\mathrm{T}_{p}}{\mathrm{~T}_{r e p}}=2 \%
$$

\section{Normalized Emittance}

The transverse geometrical emittance as defined in Eq. (35) is a constant of the motion under linear transformations. There are forces that change the emittance such as synchrotron radiation, acceleration, etc. When the particles undergo an acceleration, their phase space area are not conserved anymore since the determinant of the transfer matrix of an acceleration unit is not unity $(\operatorname{Det}(M) \neq 0)$. This phenomenon is known as adiabatic damping [6]. An invariant of motion, called normalized 
a)

I (A)

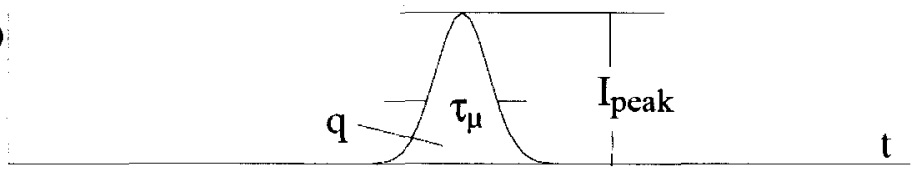

b)

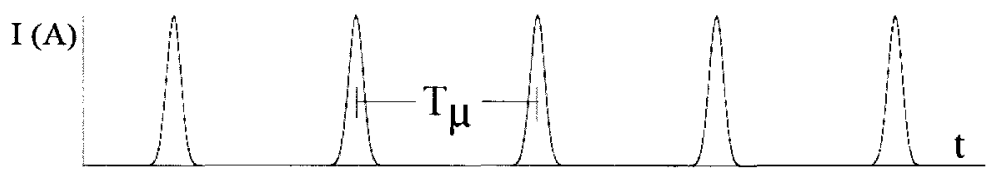

c)

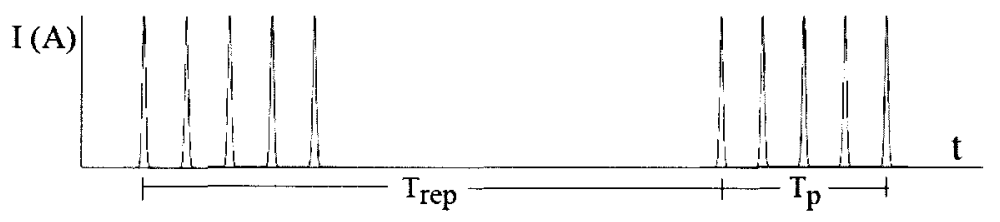

I (A)

d)

FIG. 7: In this figure [6], micro and macro beam current definitions and related time structures are illustrated; (a) Peak current $(I)$ (b) Pulse current $\left(I_{p}\right)$ (c) Average current $\langle I\rangle$ (d) Continuous current $(I)$.

emittance $\left(\varepsilon_{N}\right)$, with the presence of linear accelerators is a conserved quantity given as:

$$
\varepsilon_{N}=\gamma \beta \varepsilon
$$

where $\gamma$ and $\beta$ are relativistic factors. It is worth to note that even the normalized emittance is not conserved anymore if there are dissipating processes such as synchrotron radiation, scattering, etc.

\section{Emittance Growth}

As explained in the normalized emittance definition Liouville's theorem states that the emittance is a constant for a conservative system [6] if the forces acting on the beam are linear and conservative. Some processes contribute to the emittance growth and lead to filamentation of the distribution in the phase-space area. The main causes of emittance growth are; non-linear forces, high-order magnetic fields, field errors, beam mismatch, chromatic effects, etc. [15]. 


\section{Brightness}

The Brightness of the beam is defined as; amount of current (charge) passing per unit squared phase space area (in $4 \mathrm{D}$ space). The area is the product of $\varepsilon_{x}$ and $\varepsilon_{y}$ transverse phase space areas. Brightness is a tool to measure the quality of the beam, which shows how well the beam is collimated and focused into a small spot size. The brightness is given as:

$$
\mathbf{B} \propto \frac{I}{\varepsilon_{x} \varepsilon_{y}} \propto \frac{\text { Number of particles }}{\varepsilon_{x} \varepsilon_{y}}
$$

where $I$ is the current and $\varepsilon_{x}-\varepsilon_{y}$ are the transverse emittances. 


\section{I.3 BASIC DESCRIPTION OF CEBAF}

The Continuous Electron Beam Accelerator Facility (CEBAF) project started in mid 1980s where the construction began in 1987. Later, in 1996 the name of the laboratory was changed to Thomas Jefferson National Accelerator Facility (TJNAF) (a.k.a Jefferson Lab). Originally designed as a $4 \mathrm{GeV}$ machine, though it was left flexible to be upgraded to higher energies, technological improvements led the machine to achieve $6 \mathrm{GeV}$ beam energy. The lab has three user halls to do electron scattering experiments; Hall A, Hall B and Hall C. Another major component of Jefferson Lab is the Free Electron Laser (FEL) which the construction started in 1996. FEL achieved the highest power of light amongst its equivalents in 1998. Jefferson Lab was on of the pioneer labs in super-conducting technology (SRF) for accelerating electrons in a highly efficient, cost-effective way when compared to other institutions. The CWSRF beams of CEBAF are of unprecedented precision and stability. The majority of the present nuclear physics research program at JLAB would be impossible with conventional RF beams. In addition, in the FEL, energy recovery is used, such that $99 \%$ of the power in the beam is recycled.

Another milestone in the laboratory's history is the $12 \mathrm{GeV}$ upgrade, which the current beam energy will be doubled upon successful completion of the project. The upgrade consists of almost all existing magnet and electronics infrastructure modified and reused. In addition to that, new improved cryomodules (house for the SRF cavities) will be added to double the beam energy. Another end user hall, Hall D, will also be added to serve the scientific needs.

JLAB beam has earned reputation and cited as many times for the beam quality achieved today. Currently, it is possible to deliver the beam simultaneously to three halls. The electron beam polarization is at an unprecedented $80 \%$ ratio. A summary of the beam feature lists for present status as well as $12 \mathrm{GeV}$ upgrade is shown in Table 2.

The CEBAF beamline diagram is shown in Fig. 8. The diagram shows the basic components of CEBAF. We can divide CEBAF into four main parts; Gun \& Injector, North and South Linacs, Arcs and User Halls (A, B and C). In the gun section, the electron beam is continuously emitted from two electron guns. Three Diode lasers working at $499 \mathrm{MHz}$ each with a phase difference $\Delta \phi=120^{\circ}$ make it possible to deliver beam simultaneously to the three user halls. Bunchers are positioned after the guns to control the bunch length of the beam. Followed by choppers, cryomodules, 
TABLE 2: CEBAF beam parameters in $6 \mathrm{GeV}$ and $12 \mathrm{GeV}$ upgrade [16, 17].

\begin{tabular}{lccc}
\hline \hline Item & Unit & Present & $12 \mathrm{GeV}$ \\
\hline Injected beam energy & $\mathrm{GeV}$ & 0.065 & 0.126 \\
Maximum delivered energy & $\mathrm{GeV}$ & 6.0 & 12.0 \\
Normalized $r m s$ emittance $\left(\varepsilon_{N}\right)$ & $\mathrm{mm} \cdot \mathrm{mrad}$ & 1.0 & 1.0 \\
Fractional energy spread & $\delta$ & $<10^{-4}$ & $<10^{-4}$ \\
Transverse $r m s$ beam size & $\mu \mathrm{m}$ & $50-100$ & $50-100$ \\
Longitudinal rms Beam Size & $\mu \mathrm{m}(\mathrm{fsec})$ & $60(200)$ & $60(200)$ \\
Duty factor & & $\mathrm{CW}$ & $\mathrm{CW}$ \\
Max. summed current to Halls A\&C & $\mu \mathrm{A}$ & 180 & 85 \\
Max. summed current to Halls B (D) & $\mu \mathrm{A}$ & 0.2 & $5(5)$ \\
\hline \hline
\end{tabular}

and finally the injector chicane, where the beam is injected into the first linac, the North Linac (NL). At the end of the NL, the electron beam energy has increased from $\sim 65 \mathrm{MeV}$ to $\sim 665 \mathrm{MeV}$. The ARCs divert the beam by $180^{\circ}$. After the first arc (ARC1) the beam is injected into the South Linac (SL), where it gets the same amount of energy $0.6 \mathrm{GeV}$. The beam is again diverted $180^{\circ}$ by the West Arc dipoles completing its first pass. The beam continues to travel, where at the 5th pass it reaches to $6 \mathrm{GeV}$. Here the RF separators, working at $499 \mathrm{MHz}$ subfrequency, deliver the beam to the user halls. Up to $180 \mu \mathrm{A}$ of electron beam current can be delivered to either Hall A or Hall $\mathrm{C}$ and between $0.2-200 \mathrm{nA}$ to Hall $\mathrm{B}$. The normalized emittance of the beam is $\varepsilon_{N}=1 \mathrm{~mm} \cdot \mathrm{mrad}$ as measured in the injector. The longitudinal beam size is measured $200 \mathrm{fs}$ (or $60 \mu \mathrm{m}$ ). The transverse spot size is between 50-100 $\mu \mathrm{m}$ depending on the location. In Fig. 8, a general schematic of the CEBAF is shown. This illustrates what changes the $12 \mathrm{GeV}$ upgrade brings to the CEBAF.

In the cryomodules, 5-cell cavities are used in the present configuration. These original CEBAF cavities are $50 \mathrm{~cm}$ effective length 5-cell (C50) cavities, running at $1497 \mathrm{MHz}$ super-conducting radio frequency (SRF). C50 cavity has an original design of $5 \mathrm{MV} / \mathrm{m}$ energy gain. Recent improvements made it possible to reach $10 \mathrm{MV} / \mathrm{m}$. It is made of Niobium with an elliptical shape. There will be upgrades to the existing cryomodules, where some of them will be replaced with the new 7-cell (C100) type cavity modules. In the $12 \mathrm{GeV}$ upgrade, upgrade from 5-cell to 7-cell cavities is 
part of the major upgrade. This 7-cell cavity is the low power loss $70 \mathrm{~cm}$ effective length cavity with $1497 \mathrm{MHz} \mathrm{RF}$. It currently reaches $19 \mathrm{MV} / \mathrm{m}$ energy gain. The schematic structure of the $\mathrm{C} 50$ and $\mathrm{C} 100$ cavities are shown in Fig. 9. In addition to these replacements, ten new $\mathrm{C} 100$ cryomodules will be inserted to the free space in the linac areas. An additional arc and a new user hall, Hall D, are major parts of the upgrade process as well.

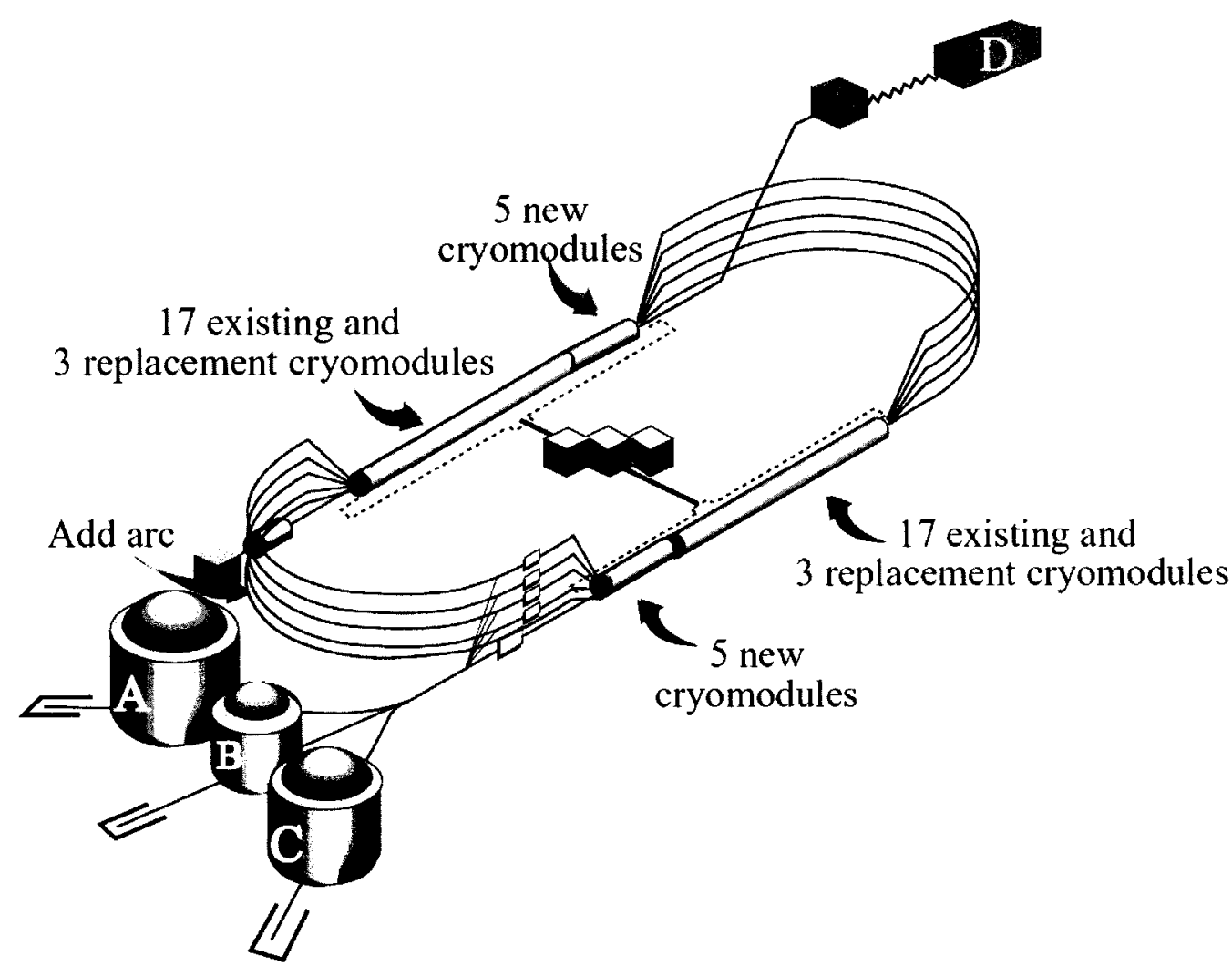

FIG. 8: The schematic drawing of the CEBAF. Also seen the 12-GeV upgrade where ten new C100 cryomodules will be added to NL and SL. Another arc line and a new over the ground user hall (Hall D) are other major updates. 


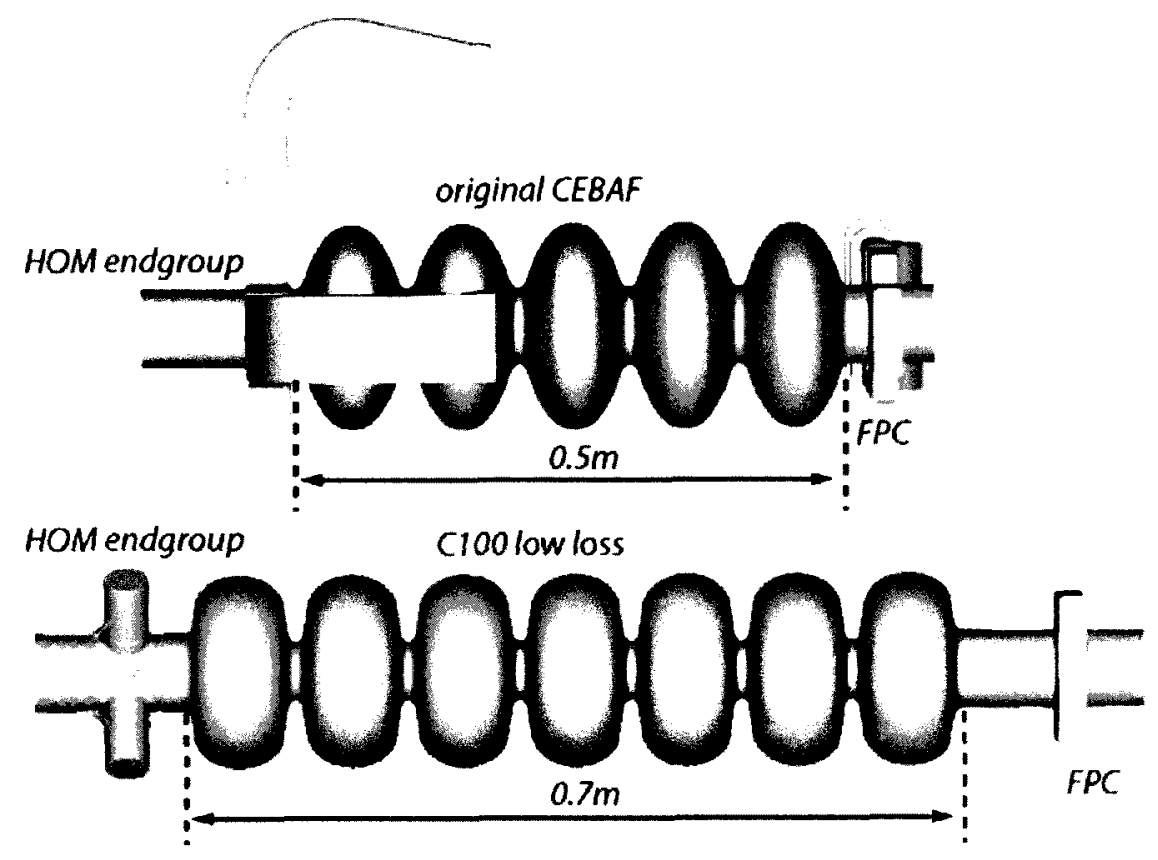

FIG. 9: (Top) The original CEBAF $50 \mathrm{~cm}$ effective length 5-cell cavity is shown. This is a $1497 \mathrm{MHz} R F$ cavity with a design of $5 \mathrm{MV} / \mathrm{m}$ energy gain. Recent improvements made it possible to reach $10 \mathrm{MV} / \mathrm{m}$. (Bottom) Upgrade from 5-cell to 7-cell cavities is envisioned in the $12-\mathrm{GeV}$ upgrade. This is the low power loss $70 \mathrm{~cm}$ effective length 7-cell cavity with $1497 \mathrm{MHz}$ RF. It currently reaches $19 \mathrm{MV} / \mathrm{m}$ energy gain. 


\section{I.4 SIMULATION TOOLS}

Simulation tools are necessary to design complex structures such as accelerator machines. They also give a greater insight by allowing detailed studies. The simulation tools I have used are OPTIM (OP) [18] mostly a linear optics code, ELEGANT (EL) [19] is a C based code, which includes high order effects and G4BEAMLINE (G4) [20] is a GEANT4 [21] based simulation software capable of realistic simulations with ray tracing feature. G4BEAMLINE includes interaction of radiation with matter; absorption and creation of particles; multiple scattering and energy loss. It also includes realistic magnetic field maps.

Although some of these software are widely used by the scientific community and their results are well accepted, it is good practice to benchmark them to each other. As a starting trial concept, I start with simple lattices such as a single solenoid, a quadrupole triplet and 7-cell CEBAF style RF cavities. By using these lattice types, we check the twiss parameters between simulation codes. In all these benchmarks, the beam is a gaussian positron beam except for the last test (Test-7), in which the beam is non-gaussian. The central design momentum used in the simulations is $p\left(\mathrm{e}^{+}\right)=15 \mathrm{MeV} / \mathrm{c}$. I compared both design momentum for all three simulations and off-momentum beam results for ELEGANT and G4BEAMLINE as they include high-order terms and fringe effects.

In Table 3, the results of first three benchmarks are shown. In these simulation benchmarks, CEBAF quality beam is used with a normalized emittance $\gamma \beta \varepsilon=\varepsilon_{N}=$ $1.2 \mathrm{~mm} . \mathrm{mrad}$. The initial and final twiss parameters are also given in the table. The energy spread of the beam is $\delta=10^{-4}$ at $15 \mathrm{MeV} / \mathrm{c}$. In all tests, drift (D) spaces in between, prior and after the elements are $30 \mathrm{~cm}$. In Test-1, a single solenoid (S) $\left(16 \mathrm{~cm}, \mathrm{~B}_{s}=0.5 \mathrm{~T}, 32 \mathrm{~A} / \mathrm{mm}^{2}\right)$ is used. The lattice structure is D-S-D. In Test-2 quadrupole (Q) triplet is benchmarked, where the lattice structure is $\mathbf{D}-\mathbf{Q}_{F^{-}}$ D-Q ${ }_{D}-\mathbf{D}-\mathbf{Q}_{F}-\mathbf{D}$. In Test-3, a C100 style $1497 \mathrm{MHz}$ quarter cryomodule (RF) is benchmarked, with D-RF-D-RF-D. The benchmark results are in almost perfect agreement for these tests. There are negligible differences with G4 results in all test. This is due to the fact that, EL and OP are matrix calculation codes and for example they use magnetic fields as inputs, which G4 uses coils for the solenoid and this makes a difference in fringe field calculations.

In Table 4, benchmark Test- 4 results are presented. In this test, we simulated a single solenoid but this time the beam has almost 100 times larger emittance. Both 
TABLE 3: Comparison of G4BEAMLINE (G4), OPTIM (OP) and ELEGANT (EL) twiss parameter results at the end of various magnets. The beam used in all simulations are gaussian beams with a normalized emittance $\varepsilon_{N}=1.2 \mathrm{~mm} . \mathrm{mrad}$.

\begin{tabular}{llrrrrrrrrrr}
\hline \hline Benchmarks & & \multicolumn{3}{c}{$\begin{array}{c}\text { Test-1 } \\
\text { Solenoid }\end{array}$} & \multicolumn{4}{c}{$\begin{array}{c}\text { Test-2 } \\
\text { Quad Triplet }\end{array}$} & \multicolumn{4}{c}{ Test-3 } \\
& & \multicolumn{3}{c}{ RF } \\
\hline Twiss par. & Initial & OP & EL & G4 & OP & EL & G4 & OP & EL & G4 \\
\hline$\beta_{x}(\mathrm{~m})$ & 2.0 & 0.09 & 0.09 & 0.09 & 0.4 & 0.4 & 0.3 & 3.2 & 3.2 & 3.5 \\
$\beta_{y}(\mathrm{~m})$ & 2.0 & 0.09 & 0.09 & 0.09 & 0.7 & 0.7 & 0.7 & 3.2 & 3.2 & 4.0 \\
$\alpha_{x}$ & 0.0 & 0.3 & 0.3 & 0.2 & 0.9 & 0.9 & 0.8 & -0.5 & -0.5 & -0.2 \\
$\alpha_{y}$ & 0.0 & 0.3 & 0.3 & 0.2 & -0.4 & -0.4 & -0.5 & -0.5 & -0.5 & -0.3 \\
$\varepsilon_{x}$ (mm.mrad) & 0.04 & 0.04 & 0.04 & 0.04 & 0.04 & 0.04 & 0.04 & 0.016 & 0.016 & 0.016 \\
$\varepsilon_{y}$ (mm.mrad) & 0.04 & 0.04 & 0.04 & 0.04 & 0.04 & 0.04 & 0.04 & 0.016 & 0.016 & 0.016 \\
\hline \hline
\end{tabular}

on-momentum $\delta=0$ and large energy spread $\delta=0.033 \sim 3 \%$ tests are completed for the codes. The accuracy of EL and G4 is considered for off-momentum particles. Lattice is $\mathrm{S}-\mathrm{D}$ where the solenoid field is $\mathrm{B}_{s}=1 \mathrm{~T}, 16 \mathrm{~cm}$ length. To get equivalent magnetic field in the G4 simulation, a $16 \mathrm{~cm}$ coil with $57 \mathrm{~A} / \mathrm{mm}^{2}$ with $20 \mathrm{~mm}$ aperture is used. When the beam has zero energy spread $\delta=0$, all codes are in very good agreement. No emittance growth is seen. But with the fractional energy spread at $\delta=3 \%$, the emittance grows by a little bit more than a factor of two in EL and G4. As it seen, this is due to the non-linear chromatic effects of the beam and from the magnet where large angles at different energies get different kicks resulting in the emittance growth.

In Table 5, quadrupole triplet benchmark results are presented, where the lattice configuration is $\left(\mathbf{D}-\mathrm{Q}_{F}-\mathrm{D}-\mathrm{Q}_{D^{-}} \mathbf{D}-\mathbf{Q}_{F^{-}}-\mathrm{D}\right)$. The initial and final twiss parameters are given in the table, where the beam momentum is $15 \mathrm{MeV} / \mathrm{c}$. Here again, due to the chromatic effects we get emittance growth in both G4 and EL, while the twiss parameters at the end of the lattice are in close agreement.

In Table 6, magnet configuration with dipoles (B) added to the triplet-solenoid system is presented (S-D- $\left.\mathbf{Q}_{F}-\mathrm{D}-\mathrm{Q}_{D}-\mathrm{D}-\mathrm{Q}_{F}-\mathrm{D}-\mathrm{B}-\mathrm{D}-\mathrm{Q}_{F}-\mathrm{B}-\mathrm{D}\right)$. The bending dipoles are formed of achromatic structure. At $\delta=0$, there is a slight disagreement between the codes, while at $\delta=3 \%$, EL and G4 are different by a factor of 2-3 from their 
TABLE 4: Comparison of simulation's twiss parameters at the end of a solenoid. The beam used in all simulations are gaussian beams at $15 \mathrm{MeV} / \mathrm{c}$ central momentum.

\begin{tabular}{lcccccc}
\hline \hline Test-4 & \multicolumn{3}{c}{$\delta=0$} & \multicolumn{2}{c}{$\delta=3.3 \%$} \\
Solenoid & Initial & OP & EL & G4 & EL & G4 \\
\hline$\beta_{x}(\mathrm{~m})$ & 0.003 & 3.5 & 3.5 & 3.4 & 1.8 & 1.8 \\
$\beta_{y}(\mathrm{~m})$ & 0.003 & 3.5 & 3.5 & 3.3 & 1.8 & 1.8 \\
$\alpha_{x}$ & 0.0 & -0.1 & -0.1 & -0.1 & -0.2 & -0.1 \\
$\alpha_{y}$ & 0.0 & -0.1 & -0.1 & -0.1 & -0.2 & -0.1 \\
$\varepsilon_{x}$ (mm.mrad) & 5.0 & 5.0 & 5.0 & 5.0 & 11.0 & 10.0 \\
$\varepsilon_{y}(\mathrm{~mm} . \mathrm{mrad})$ & 5.0 & 5.0 & 5.0 & 5.0 & 11.0 & 9.0 \\
\hline \hline
\end{tabular}

TABLE 5: Comparison of simulation's twiss parameters at the end of a quadrupole triplet. Quadrupole field gradients are $\mathrm{B}_{F}=1.1 \mathrm{~T} / \mathrm{m}$ and $\mathrm{B}_{D}=-0.9 \mathrm{~T} / \mathrm{m}$.

\begin{tabular}{lcccccc}
\hline \hline Test-5 & \multicolumn{3}{c}{$\delta=0$} & \multicolumn{3}{c}{$\delta=3.3 \%$} \\
Quad Triplet & Initial & OP & EL & G4 & EL & G4 \\
\hline$\beta_{x}(\mathrm{~m})$ & 0.03 & 0.9 & 0.9 & 0.9 & 0.9 & 0.7 \\
$\beta_{y}(\mathrm{~m})$ & 0.03 & 0.5 & 0.5 & 0.5 & 0.5 & 0.9 \\
$\alpha_{x}$ & 0.0 & 2.0 & 2.0 & 2.0 & 2.0 & 1.5 \\
$\alpha_{y}$ & 0.0 & -0.1 & -0.1 & 0.0 & -0.1 & -0.4 \\
$\varepsilon_{x}$ (mm.mrad) & 0.5 & 0.5 & 0.5 & 0.5 & 1.0 & 0.7 \\
$\varepsilon_{y}$ (mm.mrad) & 0.5 & 0.5 & 0.5 & 0.5 & 1.0 & 1.1 \\
\hline \hline
\end{tabular}

$\delta=0$ values. The emittance growth is about a factor of three.

In Fig. 10, the lattice configuration and the $\beta$ and $\eta_{x}$ functions of the Test-6 are plotted. The lattice is type of achromatic lattice. In Figs. 11(a) and 11(b), the $x-p$ graphs of the beam at the end of Test- 5 and 6 lattices are plotted. The narrow line shown with dots is the result when $\delta=0$, the filled circles represent when $\delta=0.033 \sim 3 \%$ energy spread. It is shown that off-momentum particles tend to spread out in the quadrupoles due to the chromatic effects. The transverse offset due to the non-linear effects in the triplet test is $\Delta x=30 \%$, and in Test- 6 the offset increased by $80 \%$ when compared to its on-momentum position. 
TABLE 6: Comparison of simulation's twiss parameters at the end of a S-QQQ-BQB lattice configuration (drift spaces between each element). Momentum of the beam is $15 \mathrm{MeV} / \mathrm{c}$. Same solenoid and quadrupole strengths are used, where each dipole bends by +10 degrees.

\begin{tabular}{lcccccc}
\hline \hline Test-6 & & \multicolumn{3}{c}{$\delta=0$} & \multicolumn{2}{c}{$\delta=3.3 \%$} \\
Sol. - Q.Trip. - Dip. & Initial & OP & EL & G4 & EL & G4 \\
\hline$\beta_{x}(\mathrm{~m})$ & 0.003 & 4.5 & 4.5 & 4.0 & 2.7 & 1.0 \\
$\beta_{y}(\mathrm{~m})$ & 0.003 & 13.0 & 13.0 & 14.0 & 60.0 & 30.0 \\
$\alpha_{x}$ & 0.0 & -4.5 & -4.5 & -4.0 & -4.0 & -2.0 \\
$\alpha_{y}$ & 0.0 & -16.0 & -16.0 & -18.0 & -70.0 & -40.0 \\
$\varepsilon_{x}(\mathrm{~mm} . \mathrm{mrad})$ & 5.0 & 5.0 & 5.0 & 5.0 & 16.0 & 15.0 \\
$\varepsilon_{y}(\mathrm{~mm} . \mathrm{mrad})$ & 5.0 & 5.0 & 5.0 & 5.0 & 17.0 & 15.0 \\
\hline \hline
\end{tabular}

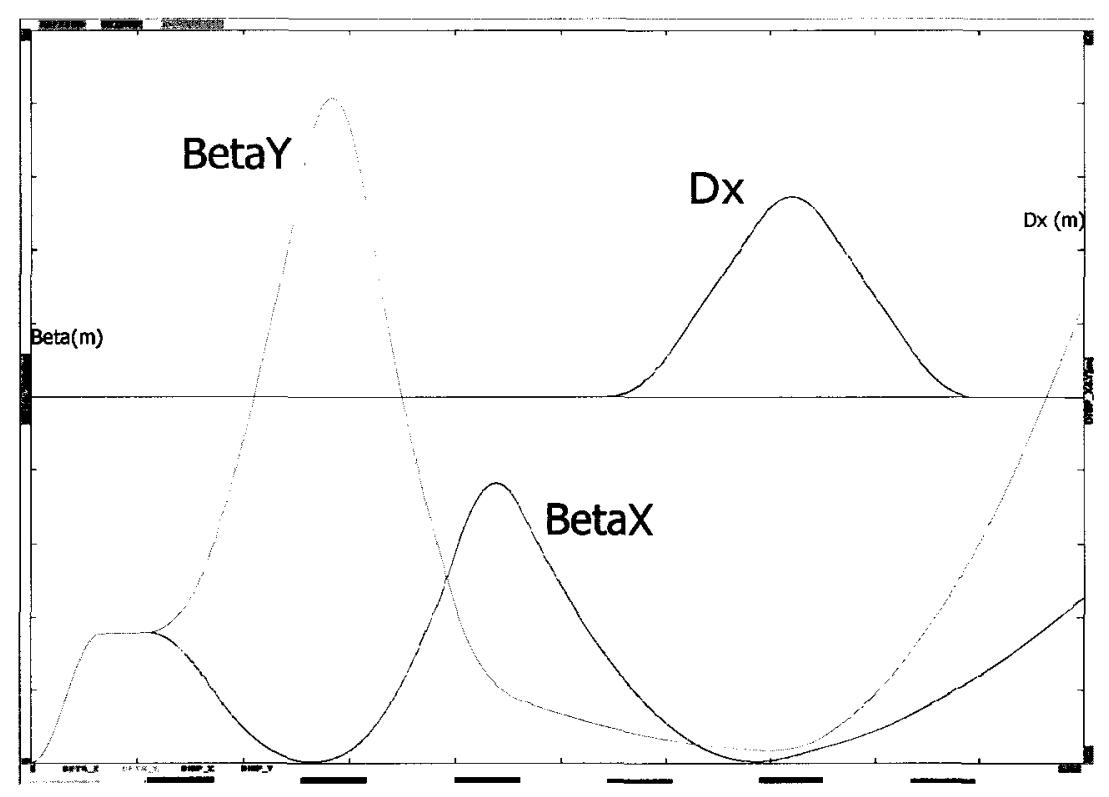

FIG. 10: In the horizontal layout magnet positions are shown from left to right S-QQ-Q-D-Q-D respectively. In the graph the twiss beta BetaX $\left(\beta_{x}\right)$, BetaY $\left(\beta_{y}\right)$ and dispersion functions Dx $\left(\eta_{x}\right)$ are plotted for Test-6. 


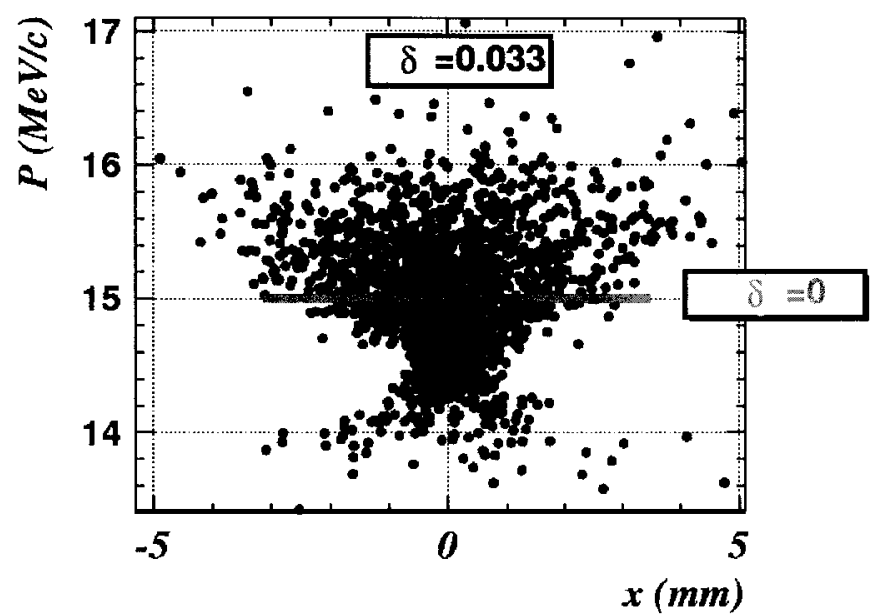

(a)

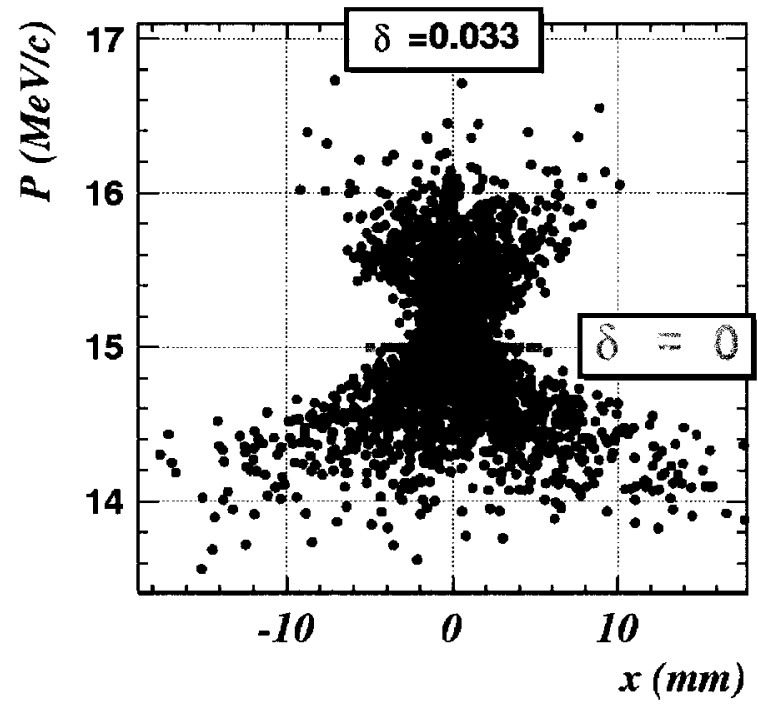

(b)

FIG. 11: (a) The $x-p$ plot of the beam at the end of Test-5 where a quadrupole triplet is used. (b) $x-p$ plot of the beam at the end of Test- 6 lattice where solenoid-quad triplet-dipole set is used. 


\section{Test-7 - Simulation with a non-gaussian beam}

A more complex lattice structure, which is composed of a solenoid, quadrupoles and RF units, is also benchmarked. But this time a non-gaussian distribution (hardedge) is used. The transverse $\beta$ functions are shown in Fig. 12 for three simulation codes. It should be noted that the beam used in this plot for G4 is the Monte-Carlo generated positron beam via pair creation. This test beam is a hard-cut selection from a non-gaussian output in G4. In OP and EL, the initial values used are the ones obtained from this generated output. The beam has the same fractional energy spread $|\delta|=3.3 \%$ at $15 \pm 0.5 \mathrm{MeV} / \mathrm{c}$ (r.m.s), where the initial twiss parameters are $\beta_{x}=\beta_{y}=0.003 \mathrm{~m}, \alpha_{x}=\alpha_{y}=-0.1$ and $\varepsilon_{x}=\varepsilon_{y} \sim 5.8 \mathrm{~mm} . \mathrm{mrad}$. With this non-gaussian beam, and large energy spread it is very complicated to match the twiss parameters. As it can be seen in Fig. 12, the $\beta$ functions show very close results where OP and EL almost match each other. The G4 results are slightly different than $\mathrm{OP}$ and EL $\beta$ functions. To match the G4 results to OP and EL, the G4 magnets are optimized (tuned) to match the twiss parameters of the EL at each quadrupole magnet.

\section{G4BEAMLINE Elements}

To give a more detailed information about the visual and physical capabilities of G4 a snapshot from the simulation is shown in Fig. 13. G4 is a ray tracing software, which also has the capability to interact with GEANT4 elements. In the figure, the solid lines passing through elements are actually the trailings of the $\mathrm{e}^{+}$. In addition to creation of particles via monte carlo simulations, it can build basic physical structures such as cylindrical type solid quadrupole, a sector dipole and a pillbox RF cavity with tunable phase and timing arguments. By having the physical structure built, one can realistically lose particles and have them deposit their energies in those elements. In Fig. 13(a), a sector bending dipole is shown in a solid form. In Fig. 13(b), a quadrupole doublet is shown in wired form where you can see the beam orbit inside the magnetic element as well. In Fig. 13(c), the C100 RF unit is shown. This is not exactly the same shape with the actual C100 unit, which in the simulations seven cylindrical pillboxes are used to simulate $\mathrm{C} 100$ as shown in the figure . 


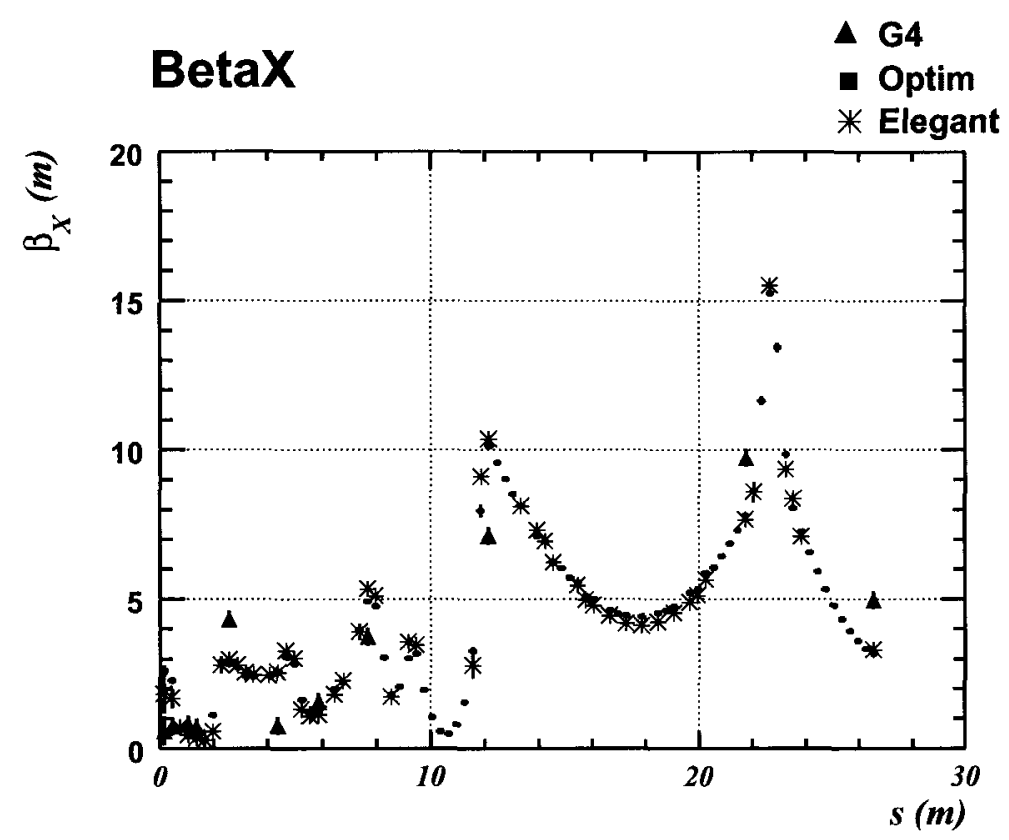

(a)

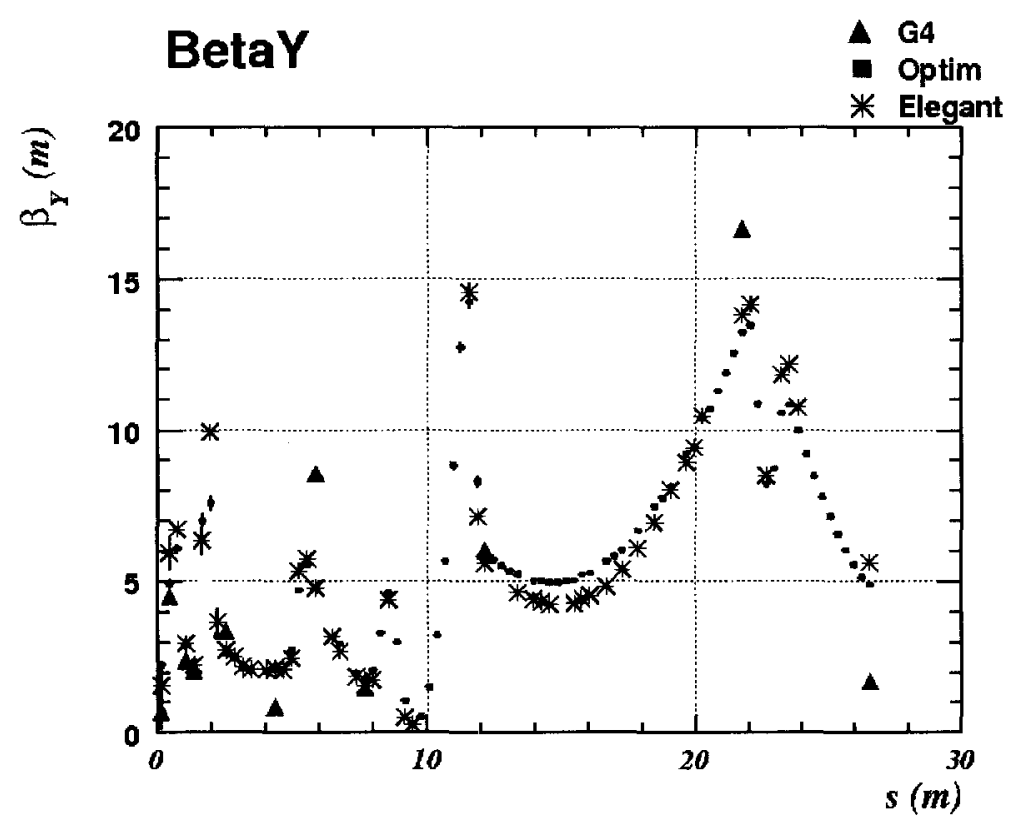

(b)

FIG. 12: (a) The $\beta_{x}$ of a non-gaussian flat distribution (flat in momentum and angle) beam tracked through three simulation codes. (b) Same with $\beta_{y}$. The $\beta$ functions are in good agreement between codes. 


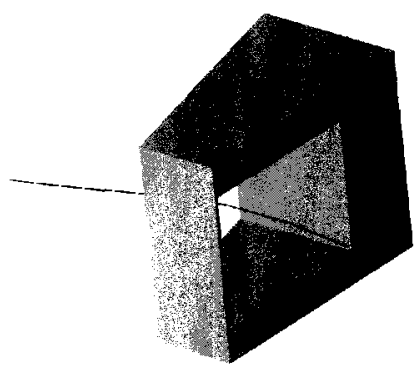

(a) Sector dipole

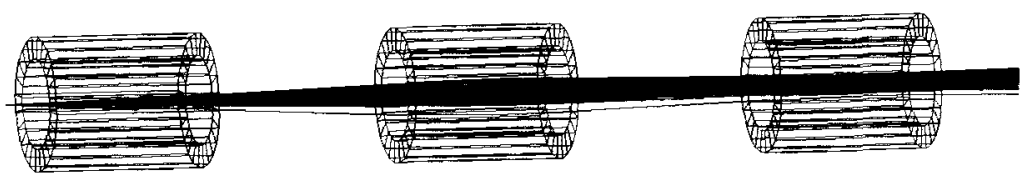

(b) Quadrupole triplet in wire mode.

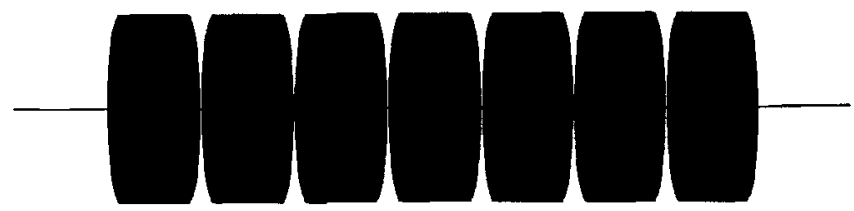

(c) C100 7-cell RF cavities are simulated with pillbox cavities in $\mathrm{G} 4$.

FIG. 13: Snapshots from the G4 simulation. It has a 3D visualisation option such that one can see the elements and trails of the particles within these elements. It is a very useful feature to investigate where the particles are lost or where they actually go. The visualisation can be set to solid elements, wire type elements or no elements but just the fields. The lines passing through elements are the traces of the positrons. (a) Sector dipole bends a $\mathrm{e}^{+}$beam. (b) Wire type visualisation of a quadrupole triplet system transforms the beam (c) C100 type 7-Cell RF unit is shown where the RF is simulated with cylindrical pillbox cavities. 


\section{CHAPTER II POSITRON SOURCES}

Positrons are created either through electromagnetic interactions, or within the nucleus via the weak interaction (spontaneous $\beta^{+}$decay). In accelerator based positron sources a conventional positron source consists in a high- $Z$ target hit by a primary electron beam. Then photons, which are produced by bremsstrahlung, are converted in the same target into $\mathrm{e}^{+} \mathrm{e}^{-}$pairs resulting in an electromagnetic shower. In Fig. 14, the bremsstrahlung and pair production processes are illustrated by Feynman diagrams. The incoming electron traverses the converter material where it interacts with the nuclei via Coulomb force and then a photon is emitted. This emitted photon then interacts with a nucleus and is converted into $\mathrm{e}^{+} \mathrm{e}^{-}$pairs only if the photon has at least two times the rest mass energy of the electron which is $1.024 \mathrm{MeV}$. In this pair production process the energy is conserved but the kinetic energy is not necessarily divided equally between electrons and positrons. In addition, a small fraction of the energy is lost to atomic recoil.

Not all of the photons are converted into $\mathrm{e}^{+} \mathrm{e}^{-}$pairs in this process. The positron conversion cross section is dependent on the energy of the incoming electron, target material and target thickness. The thickness of the chosen target is optimized such that the number of positrons per the downstream machine acceptance is maximized and the deposited power in the target is minimized. But such a positron beam represents only a small fraction of all charged particles created in the target. This is due to their large transverse and longitudinal momentum spread, which are caused by the photon emission and pair production processes, and most importantly dominated by the multiple scattering of charged particles. This chapter will be devoted to the linac based $\mathrm{e}^{+}$sources, which have been the desire for a high intensity $\mathrm{e}^{+}$source at CEBAF. 


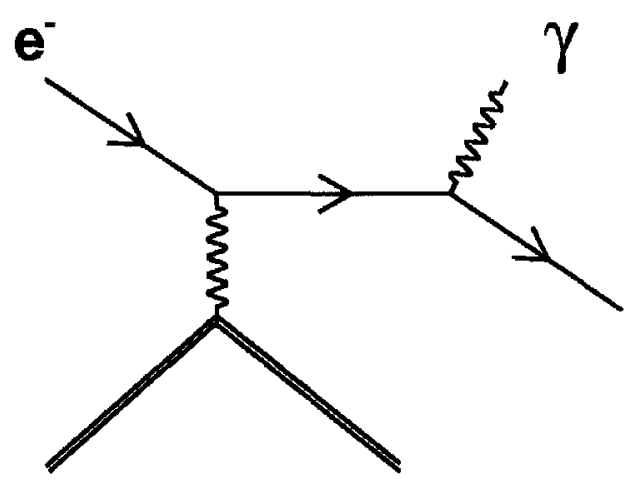

(a) Bremsstrahlung.

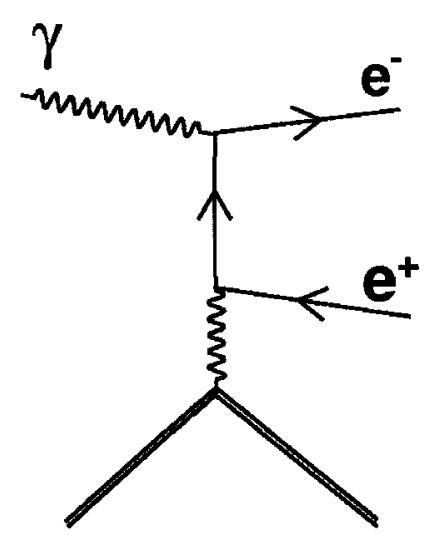

(b) Pair production.

FIG. 14: (a) Bremsstrahlung process of an electron when passing by an atomic nuclei where a photon is emitted as a result of this process (b) The emitted photon then interacts with the electric field via Coulomb interaction with the nuclei. The photon is converted into an $\mathrm{e}^{+}-\mathrm{e}^{-}$pair.

\section{II.1 NON-ACCELERATOR POSITRON SOURCES}

Another method for creating a positron beam is by using an isotope such as Sodium$22\left({ }^{22} \mathrm{Na}\right) .{ }^{22} \mathrm{Na}$ emits positive beta particles $\left(\beta^{+}\right)$, when a proton in a nucleus is converted to a neutron by emitting a positron and a neutrino as ${ }^{22} \mathrm{Na} \rightarrow{ }^{22} \mathrm{Ne}+\beta^{+}$ $+\mathbf{v}_{e}+\gamma$ with a half-life of 2.6 years. It has been demonstrated [22] that positrons from a $70 \mathrm{mCi} \mathrm{Na}-22$ source, can be accumulated at a rate of $10^{6} / \mathrm{s}$ in a Penning trap. The strength of these sources is restricted to about $100 \mathrm{mCi}$ in regular laboratory conditions. A moderator, such as a tungsten mesh or a rare gas mixture, is generally used to slow down and manipulate the fast positrons to the desired energy band. The increase in the moderated number of positrons is seen possible with solid noble gas moderators at cryogenic temperatures. But the lifetime of the moderators are limited to 1 day due to rest gas deposition as observed in Ref [22].

Nuclear reactors are also used as positron sources. Fission reactions in the core emit neutrons and gamma rays where Cadmium (Cd) rods can be used to enhance the gamma flux. The ${ }^{113} \mathrm{Cd}(\mathrm{n}, \gamma){ }^{114} \mathrm{Cd}$ reaction produces a gamma cascade of total energy $9 \mathrm{MeV}$. Platinum [23] and Tungsten [24] are then used to convert the gamma rays and to moderate the produced positrons. A continuous yield of $10^{8} \mathrm{e}^{+} / \mathrm{s}$ was measured in Ref. [24]. 
In addition to gamma conversion, positrons can be created by neutron induced activation. This is the method for creating ${ }^{22} \mathrm{Na}$ sources. An in situ positron source can be created with $\mathrm{Cu}$ rods inserted into the core. The reaction chain is:

$$
\begin{array}{r}
{ }^{63} \mathrm{Cu}+n \rightarrow{ }^{64} \mathrm{Cu}+\gamma \\
{ }^{64} \mathrm{Cu} \rightarrow{ }^{64} \mathrm{Ni}+\gamma+\beta^{+}+\nu_{e}
\end{array}
$$

Neutron activated ${ }^{64} \mathrm{Cu}$ reactor sources [25] require renewal of the source within a few days due to 12.7 hour lifetime and exhaustion of ${ }^{63} \mathrm{Cu}$ supply.

\section{II.2 REVIEW OF ACCELERATOR BASED POSITRON SOURCES}

In this section, I review the literature and summarize the features of several characteristic positron sources and facilities around the world. This section covers positrons created at linac based accelerators. The review also includes the projected or in construction (including upgrades) of the future facilities. Since these future projects are subject to change, the parameters of these facilities may change as well. The list is compiled on as reference to the last known configurations extracted from the articles, books and websites of the said facilities.

Recently there have been numerous $R \& D$ papers and proposals seen about $\mathrm{TeV}$ scale lepton collider. Different projects include TESLA, ILC, NLC, CLIC. The International Linear Collider (ILC) which has a centre-of-mass energy of $500 \mathrm{GeV}$ has a very strong support and already been completed most of its design parameters. Another possibility is a $\mu$ collider, as muons are almost 200 times heavier than electrons (positrons), they are much less susceptible to the synchrotron radiation energy losses, and can therefore be stored in a ring.

To reach their design luminosity, the nominal design parameters for CLIC require approximately $6 \times 10^{13}$ positrons per second at the interaction point, and for ILC, $2 \times 10^{14}$ positrons per second are required. As it is shown before with radioisotope and reactor sources, the positron current as high as this is currently not achievable.

\section{II.2.1 Stanford Linear Accelerator Center (SLAC)}

The SLAC [26] linac is a 2-mile long copper cavity linac, which the construction was started in 1962 and completed in 1966. Major accelerator upgrades include the SLAC Positron Electron Accumulator Ring (SPEAR), the Positron Electron Project 
(PEP) [27], the SLAC Linear Collider (SLC) and the PEP-II B-factory [27]. All of these upgrade projects required positron beams - either stored or pulsed. The SLAC accelerator complex is pictured in Fig. 15. In addition to particle physics, the SLAC complex has been extensively used as a test bed for new accelerator techniques and facilities (e.g. ILC), including Plasma Wakefield acceleration [28]. The PEP-II $\mathrm{B}$-factory investigated $\mathrm{CP}$-violation in the B-quark sector with the BaBar detector. The PEP-II facility consisted of two independent storage rings on top of each other in the PEP tunnel as shown in Fig. 16. This design created one asymmetric electronpositron collision point at the BaBar detector. The high energy ring (HER) stores a $9 \mathrm{GeV}$ electron beam - this was an upgrade of the original PEP ring. The new low energy ring (LER) stores $3.1 \mathrm{GeV}$ positions.

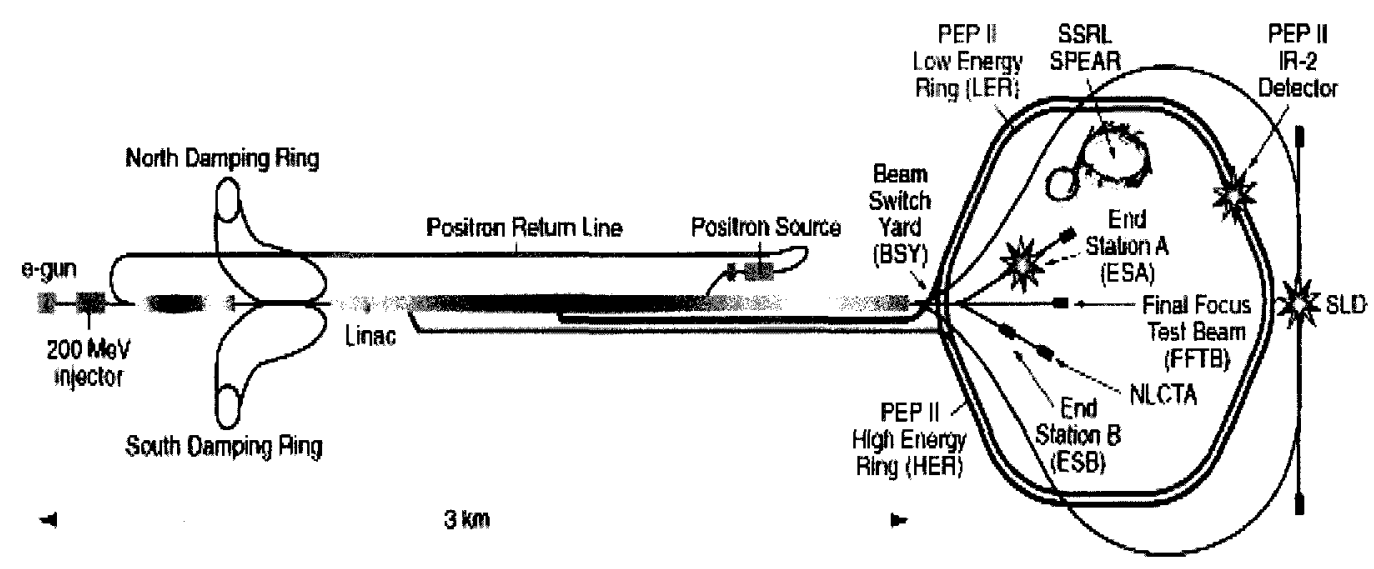

FIG. 15: The schematic layout of the SLAC ring complex is shown with the 2-mile long linac, positron source and return line, and PEP-II (B-factory) double ring collider. There are also seen Stanford Linear Collider (SLC), Final Focus Test Beam (FFTB), End Station A and B (ESA and ESB), Stanford Positron Electron Accelerating Ring (SPEAR), Stanford Synchrotron Radiation Laboratory (SSRL) and Next Linear Collider Test Area (NLCTA), which is a prototype for the Next Linear Collider (current project is called as International Linear Collider). Schematic layout is credited to the SLAC website.

The electron beam at SLAC can be accelerated up to $50 \mathrm{GeV}$ with a $120-180$ $\mathrm{Hz}$ repetition rate. At the extraction point shown in Fig.15, electrons are extracted at $33 \mathrm{GeV}$. These extracted electrons are used to create positrons. The positrons are collected in a focusing solenoid system using a tapered solenoidal field. This flux concentrator (FC) is pulsed, to produce a peak field of 5 Tesla at the production target. The schematic of the system and magnetic field profile at the flux concentrator 


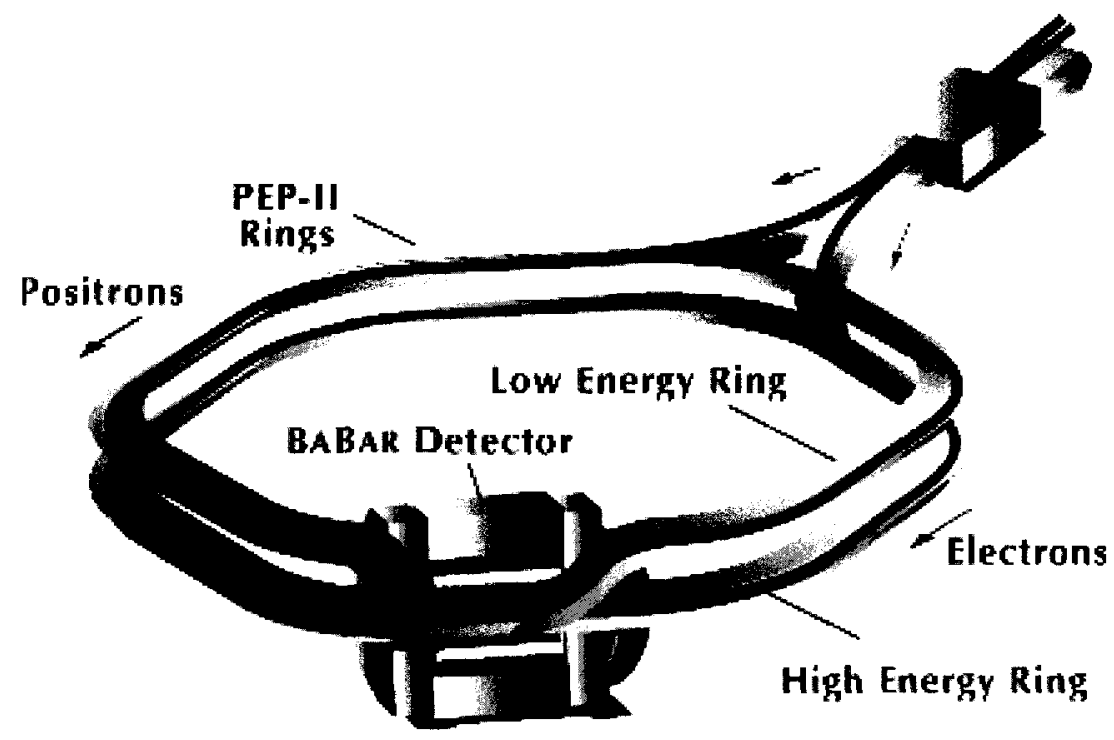

FIG. 16: PEP-II rings layout where a collision occurs in the BaBar detector between electrons and positrons. Schematic layout is credited to the SLAC website.

is shown in Fig. 17. The positron converter target is water cooled, which the cross section is shown in Fig. 18.

The positron beam emitted from the target, then captured by the tapered solenoid, where captured positrons coming out of this focusing solenoid system is then accelerated in pulsed mode to $200 \mathrm{MeV}$ in a 1.5 meter high-gradient accelerator of $50 \mathrm{MV} / \mathrm{m}$. This accelerator section is totally three units where each one is $3.05 \mathrm{~m}$ standard accelerator unit. The positrons are contained in the high-gradient accelerator aperture using a 0.5 Tesla solenoidal field followed by quadrupole focusing. A very detailed schematic, from the target to the $200 \mathrm{MeV}$ point is shown in Fig. 19. At this energy, the positrons are injected back to the beginning of the linac and accelerated up to $1.2 \mathrm{GeV}$. At this energy, they are inflected into the positron damping ring (a.k.a Sourth Damping Ring (SDR)) [29, 30]. The detailed parameters of the positron source are shown in Table. 7 .

\section{Damping Ring}

Due to the multiple scattering effects during the creation of the positrons, the emittance of the initial positron beam is too large for acceptable collider operation. Thus, 


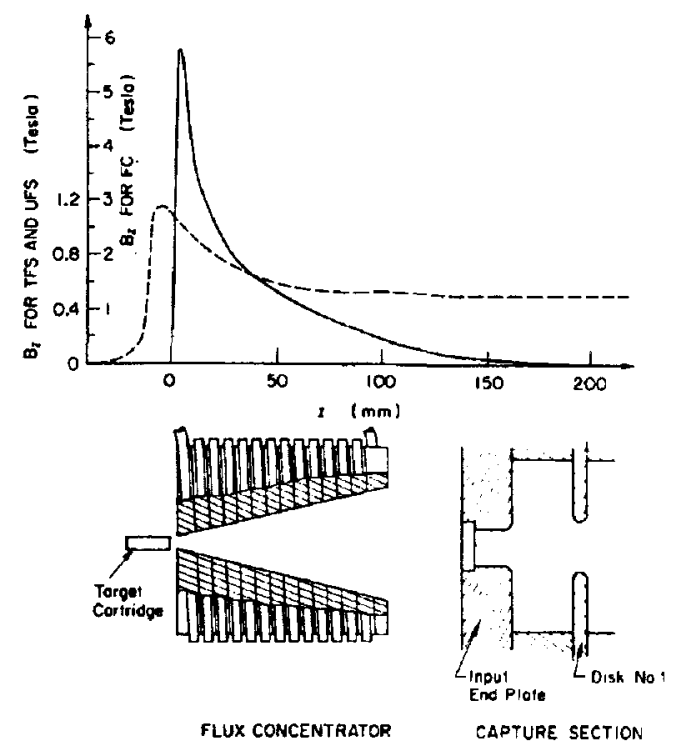

FIG. 17: The SLAC pseudo-adiabatic phase-space transformation system. The units shown in cross section at bottom are to scale. The computed DC solenoidal fields and measured Flux Concentrator (FC) pulsed field are shown above with the same z-scale [28].

each positron bunch must be collected and remain in the damping ring for a certain time to reach the desired luminosity requirements. The phase space of the positrons is damped by synchrotron radiation. With the classical approach, the power $\left(P_{\gamma}\right)$ and energy $\left(U_{0}\right)$ loss per turn are [9]:

$$
P_{\gamma}=\frac{2}{3} \frac{r_{e} c}{\left(m_{0} c^{2}\right)^{3}} \frac{E^{4}}{\rho^{2}} \quad, \quad U_{0}=\frac{4}{3} \frac{\pi r_{e}}{\left(m_{0} c^{2}\right)^{3}} \frac{E^{4}}{\rho}
$$

here $E$ is the energy of the positrons, $r_{e}$ the classical electron radius, and $\rho$ the ring bend radius. Here the energy loss per turn is calculated by multiplying the power with the revolution time $T_{r e v}=\frac{2 \pi \rho}{\beta c}$, where $\beta c$ is the speed of the particles.

The energy loss grows with the fourth power of the positron energy. Thus higher energy positrons lose energy faster, and lower energy positrons lose energy slower than the average. The average energy loss of the positron beam is compensated with $\mathrm{RF}$ acceleration. The parameters of the damping ring for the SLC positron source are listed in Table 8 [26].

The positrons stay in the damping ring approximately four radiation damping times $(\sim 12.1 \mathrm{~ms})$, which corresponds to twice the time interval between linac pulses 


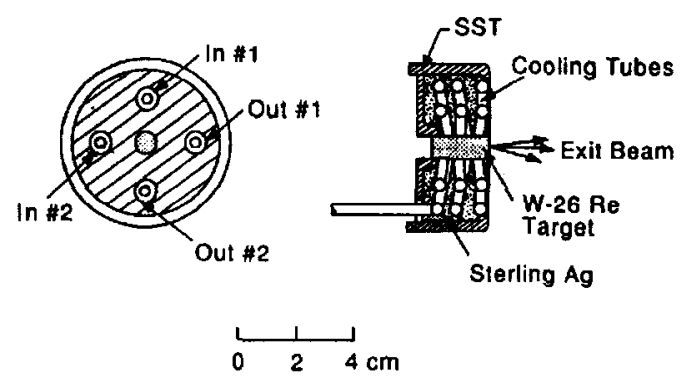

FIG. 18: Cross section of the positron converter target in SLAC [28].

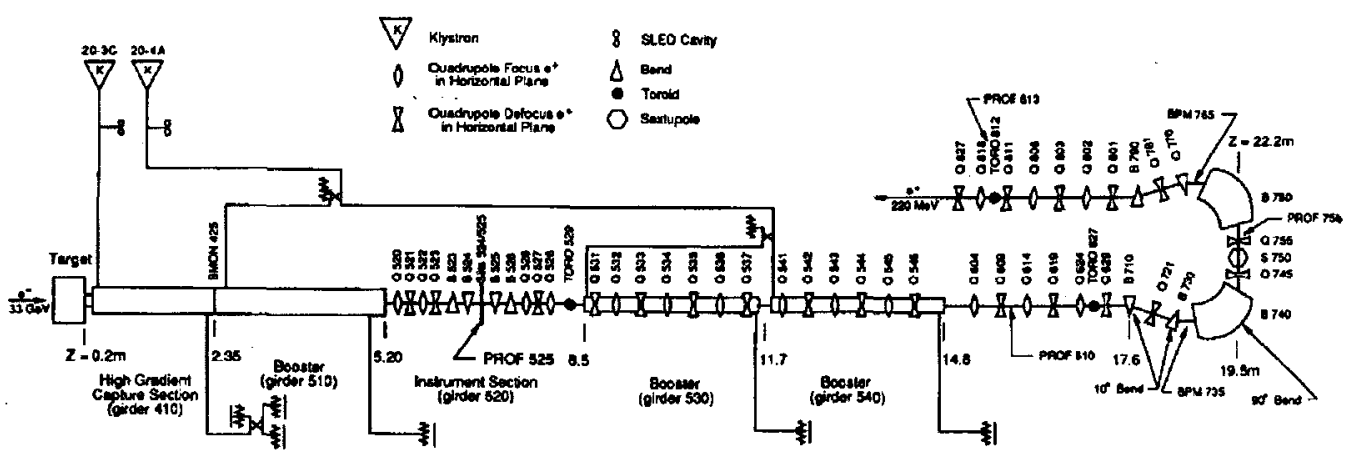

FIG. 19: The detailed drawing of the SLAC positron source, capture line, linac and $180^{\circ}$ positron return line [31]. The positron target is located on the left of the schematic, where electron beam is coming from left [28].

(at $180 \mathrm{~Hz}$ ). The positron bunch to be used in the next linac cycle is the one that is still stored in the damping ring from the previous cycle. Let us make an assumption for the CEBAF case. Assuming for a $40 \mathrm{MeV}$ positron beam with a 10 m circumference ring $(\rho=1 \mathrm{~m})$, the positrons will loose $\mathrm{U}_{0} \sim 2 \times 10^{-1} \mathrm{eV} /$ turn. But the damping time for this energy will be quite large, as the damping time can be written as $[4]$ :

$$
\tau_{d}=\frac{2 E T_{r e v}}{U_{0}} \sim 12 \mathrm{~s}
$$

So CEBAF working at $1497 \mathrm{MHz}$ in CW mode needs a damping ring which cools the beam in less than 668 ps damping time, which is technically not possible currently. 
TABLE 7: SLAC positron beam parameters and electron beam creating them $[28$, $32]$.

\begin{tabular}{|c|c|}
\hline Driving electron beam & \\
\hline Energy $(\mathrm{GeV})$ & 33.0 \\
\hline Spot Size $1 \sigma(\mathrm{mm})$ & 0.6 \\
\hline Intensity & $5 \times 10^{10} /$ pulse \\
\hline Pulse Energy (Joule/pulse) & 264.0 \\
\hline Pulse Rate $(\mathrm{Hz})$ & $120-180$ \\
\hline Beam Power $(\mathrm{kW})$ & 47.0 \\
\hline \multicolumn{2}{|l|}{ Target } \\
\hline Material & $90 \% \mathrm{Ta}-10 \% \mathrm{~W}$ and WRe \\
\hline Length (mm) & 20 (or 6 rad. length) \\
\hline Deposited Energy (Joule/pulse) & 53.0 \\
\hline Deposited Power (kW) & 9.0 \\
\hline \multicolumn{2}{|l|}{ Positron beam } \\
\hline Capture Energy $(\mathrm{MeV})$ & $5-20$ \\
\hline Spot Size $(\mathrm{mm}) 1 \sigma$ & 2.0 \\
\hline Normalized Emittance ( $\mathrm{m} \cdot \mathrm{rad})$ & $10^{-2}$ at $200 \mathrm{MeV}$ \\
\hline Yield $\left(\mathrm{e}^{+} / \mathrm{e}^{-}\right)$ & 2.5 \\
\hline
\end{tabular}

TABLE 8: SLAC positron damping ring design parameters [26].

\begin{tabular}{lr}
\hline \hline Energy (GeV) & 1.21 \\
Circumference(m) & 35.27 \\
Number of Particles / bunch & $5 \times 10^{10}$ \\
Revolution Frequency (MHz) & 8.5 \\
Transverse Damping Time (ms) & 3.059 \\
Energy Loss / turn (keV) & 93.1 \\
Bending Radius (m) & 2.037 \\
Bending Field (kG) & 19.8 \\
Acceptance of the Ring ( $\pi$ m.rad) & $\geq 1.13 \times 10^{-6}$ \\
Energy Acceptance of the Ring & $\geq \pm 1 \%$ \\
RF Frequency (MHz) & 714 \\
Harmonic Number & 84 \\
RF Voltage $(\mathrm{kV})$ & 800 \\
\hline \hline
\end{tabular}




\section{II.2.2 Beijing Electron Positron Collider (BEPCII)}

BEPCII is an electron-positron collider complex [33]. This is an upgrade from the BEPC accelerator complex, which had a $1.3 \mathrm{GeV}$ electron linac. The BEPCII accelerator has two storage rings for positrons and electrons each with a circumference of $224 \mathrm{~m}$. The beam in each ring consists of 93 bunches spaced by $8 \mathrm{~ns}$. The peak luminosity is designed to be $10^{33} \mathrm{~cm}^{-2} \mathrm{~s}^{-1}$ at the beam energy of $1.89 \mathrm{GeV}$. A luminosity of $10^{32} \mathrm{~cm}^{-2} \mathrm{~s}^{-1}$ has been reached recently [34]. The fundamental requirements to new injector linac [35] are $1.89 \mathrm{GeV}$ positron energy, the accumulated current of 50 $\mathrm{mA} / \mathrm{min}$ injection rate and very stable beam with energy spread less than $0.6 \%$. The main design parameters at the BEPC linac end were energy of $1.3 \mathrm{GeV}$, positron beam current of 4-5 mA, bunch width $2.5 \mathrm{nS}$ and repetition rate $12.5 \mathrm{~Hz}$. The electron beam energy for positron production was $140 \mathrm{MeV}$ and routine positron injection rate into the ring now was about $3 \mathrm{~mA} / \mathrm{min}$. At $\mathrm{BEPCII}, 50 \mathrm{~Hz}$ rate electrons are accelerated to $240 \mathrm{MeV}$ in the linac as an upgrade from $140 \mathrm{MeV}$, and focused to about a 1-3 mm diameter spot on a tungsten target. The target itself is a $10 \mathrm{~mm}$ diameter, $8 \mathrm{~mm}$ thick tungsten (W) disk. A SLAC type flux concentrator is used as a capture device, which is a $12 \mathrm{turn}, 10 \mathrm{~cm}$ long copper coil with a cylindrical outside radius of $53 \mathrm{~mm}$. Its inside radius is a conical type growing from $3.5 \mathrm{~mm}$ to $26 \mathrm{~mm}$. The flux modulator provides $12 \mathrm{kA}$ in a $5 \mu$ s sinusoidal half wave current at $50 \mathrm{~Hz}$ repetition rate to produce an adiabatic magnetic field profile (a.k.a AMD) with the peak of $4.5 \mathrm{~T}$ field at the flux entrance face. Downstream of the flux concentrator, there are seven DC focusing solenoid modules wrapped on the RF structures, each 1meter long with a field of $0.5 \mathrm{~T}$, to further focus and match the positron beam into the downstream quadrupole focusing system. BEPC, when first started to operate, had a positron conversion yield of $2.4 \%\left(\mathrm{e}^{+} / \mathrm{e}^{-}\right.$per $\left.\mathrm{GeV}\right)$ where the electron beam was $140 \mathrm{MeV}$ with a peak current of $2.5 \mathrm{~A}$. For this new requirement of positron injection of $50 \mathrm{~mA} / \mathrm{min}$, there have been a lot of upgrades to the linac, and capture system [35, 36]. A schematic layout of the positron source [35] is shown in Fig. 20. With the updated design, the $240 \mathrm{MeV}$ - $4.5 \mathrm{~A}$ electrons create a $80 \mathrm{~mA}$ current peak current, energy of $100 \mathrm{MeV}$ and with a conversion yield of $4.5 \% \mathrm{e}^{+} / \mathrm{e}^{-}$per $\mathrm{GeV}$, as it was measured at the positron source section exit (after the capturing RF structure). Followed by the accelerating structures, beam focusing quads and orbit correction system, a $1.89 \mathrm{GeV}$ - $61 \mathrm{~mA}$ peak current positron beam with an emittance of 1.6 $\mathrm{mm} \cdot \mathrm{mrad}(2.5 \%$ yield per $\mathrm{GeV})$ was measured at the linac exit [37]. 


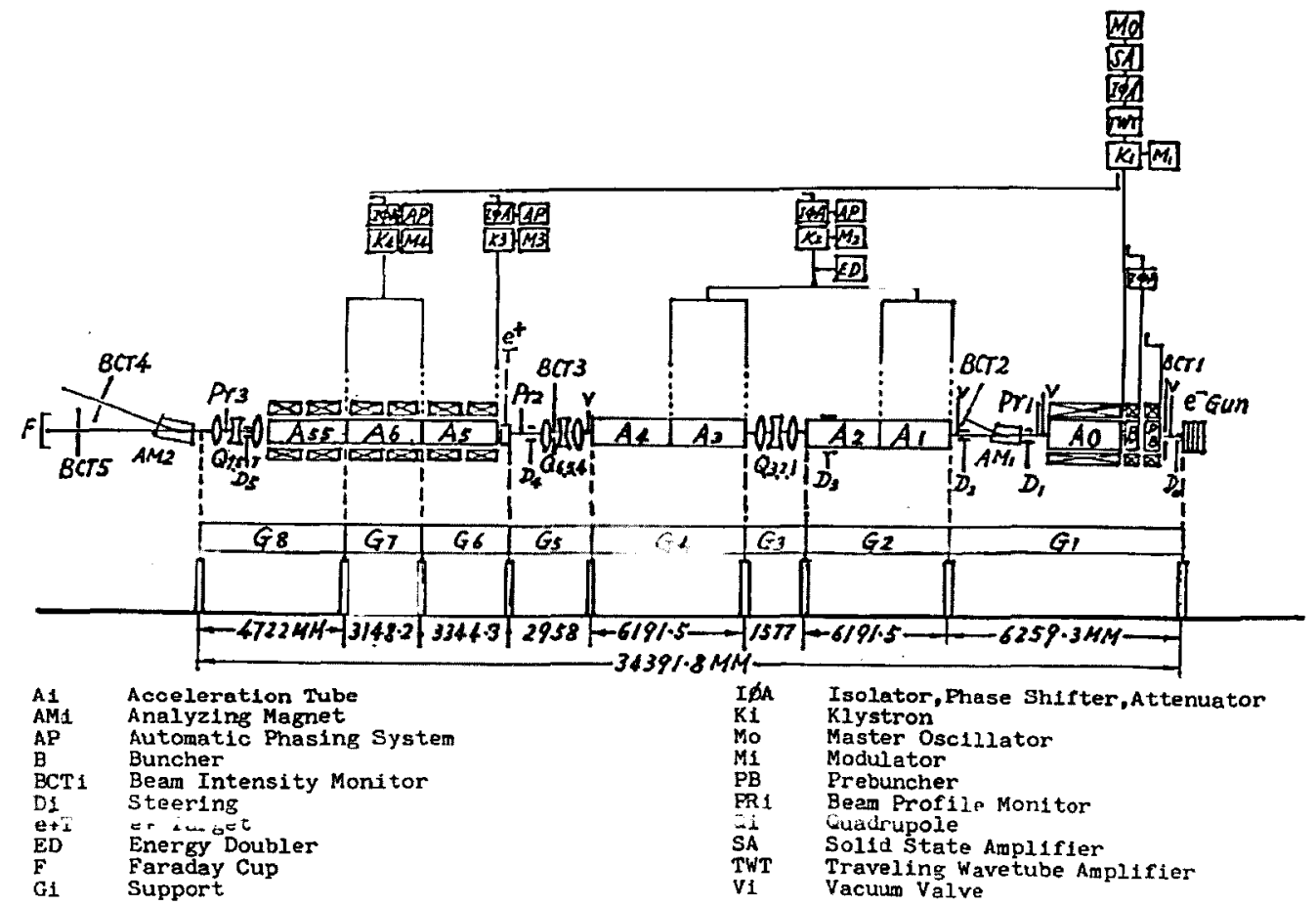

FIG. 20: The schematic layout of the BEPCII positron source. The electron beam coming from right to left starting from the electron gun. The positron converter is located between sections A4 and A5. Immediately after the positron converter, there exists a flux concentrator and a long DC solenoid surrounding an accelerator section [35].

\section{II.2.3 Cornell Electron Storage Ring (CESR)}

The Cornell Electron Storage Ring, before it ceased operation, was also an intense electron-positron collider [38, 39]. The driving electron beam energy to create positrons was $200 \mathrm{MeV}$ with a $2.5 \mathrm{~mm}$ spot size on an alloy target. The target alloy was $97 \%$ Tungsten, $2.1 \%$ Nickel and $0.9 \%$ Iron. The thickness of the target was $\mathrm{L}$ $\sim 7.063 \mathrm{~mm}$. The electron beam is pulsed with a charge of $20 \mathrm{nC} /$ pulse $\left(1.25 \times 10^{11}\right.$ $\mathrm{e}^{-}$). The positron capturing structure is the same type as in SLAC.

In Fig. 21, the positron capture system is shown. In this figure, it is shown that immediately after the conversion target, there is a pulsed flux concentrator (FC) and another long low field solenoid is positioned right after that. In Fig. 22(a), the schematic drawing of the geometry of the positron capture optics is shown. The 
cross section schematic of the FC is shown in Fig. 22(b). The FC has 16 turns in two layers of $4 \times 4 \mathrm{~mm}^{2}$ cross section oxygen free copper conductor with water cooling. The FC is followed by a bilayer solenoid, which was used as a short focussing lens. The current pulse in the lens lasts for about $25 \mu \mathrm{s}$. The positron geometrical capture efficiency was calculated to be $\sim 3.0 \%$ for $10 \mathrm{MeV}$ positrons.

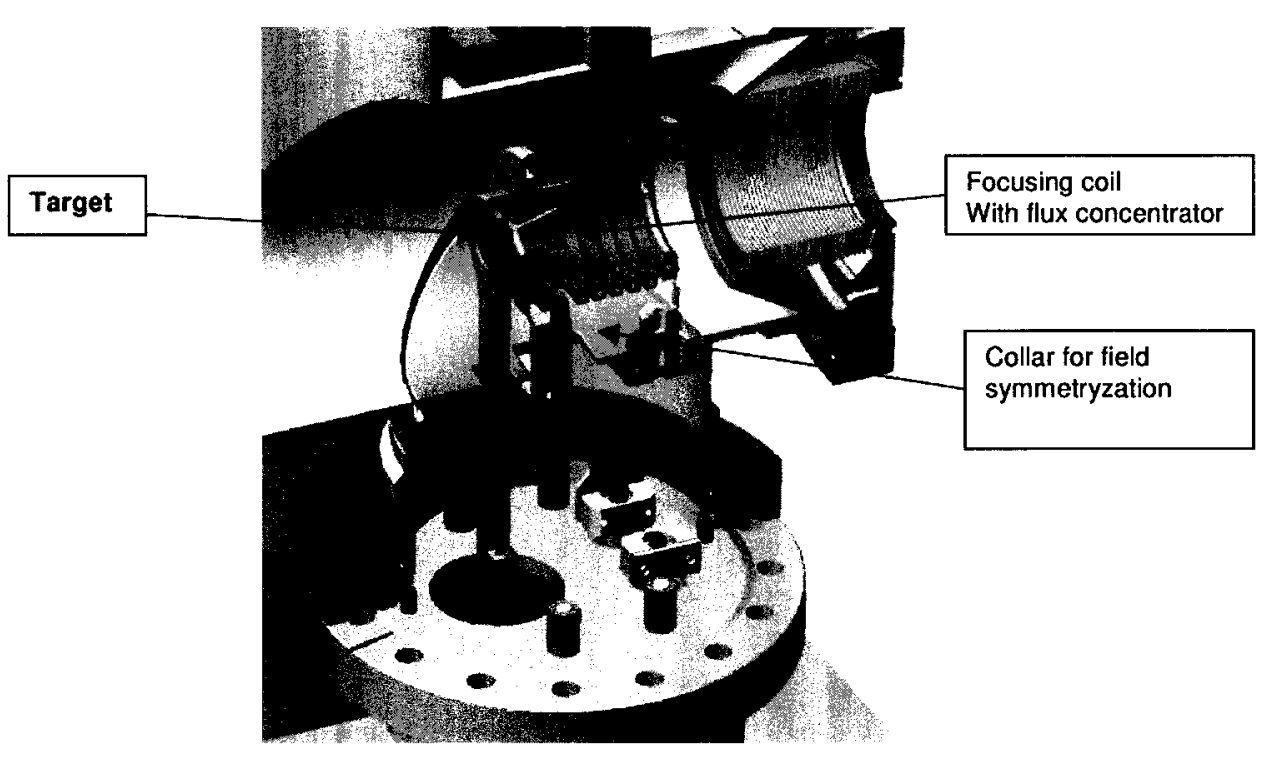

FIG. 21: An isometric view of the focusing coil. The target is fixed at the end of the paddle-type holder [38]. 


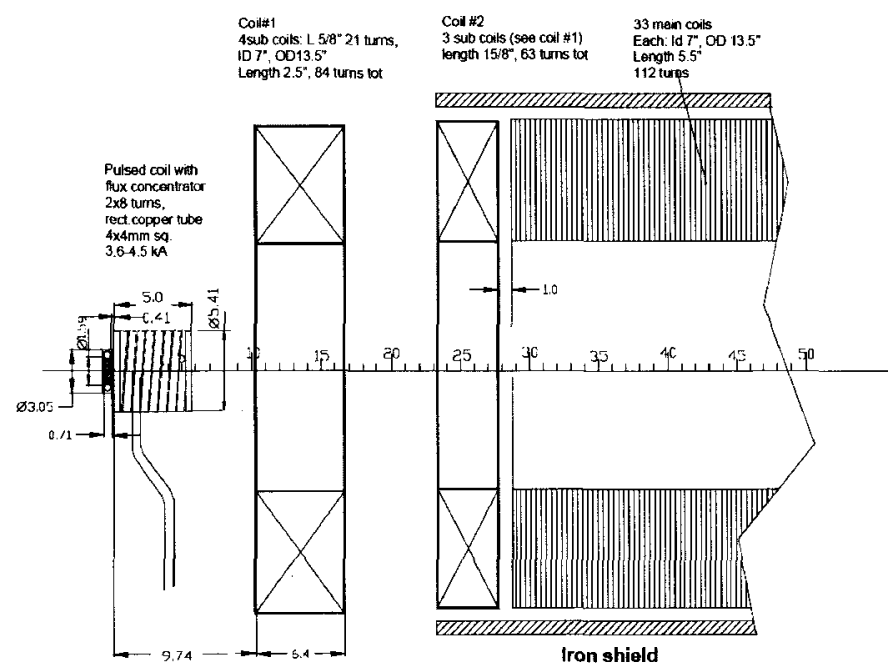

(a) Geometry of the positron capturing optics in CESR.

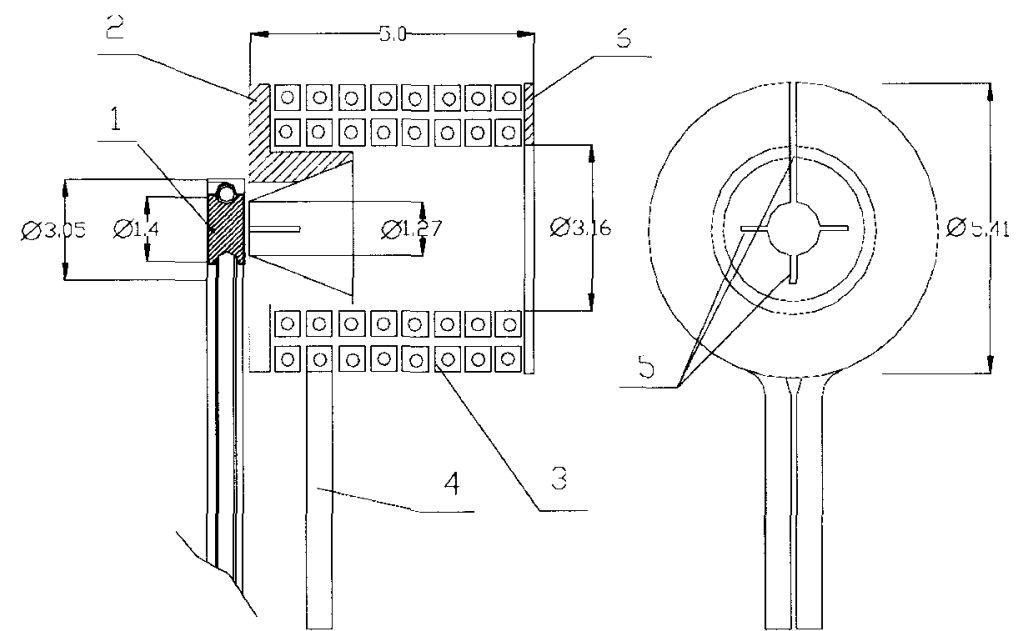

(b) Flux concentrator and other assembly

FIG. 22: (a) CESR positron converter assembly with focusing solenoid. Primary electron beam is coming from the left. (b) Scaled view of the source assembly, where: 1-Target, 2-Flux concentrator, 3-Bilayer solenoid, 4-Feeding leads, 5-Slots, 6 -End plate. Dimensions are given in $\mathrm{cm}[38]$. 


\section{II.2.4 The Budker Institute of Nuclear Physics (VEPP)}

The Budker Institute of Nuclear Physics houses the VEPP-4 complex. This includes: the linear accelerator, the booster synchrotron, the VEPP-3 storage ring and the VEPP-4M collider. The linac currently has a maximum energy of $50 \mathrm{MeV}$, and the $\mathrm{e}^{+} \mathrm{e}^{-}$booster synchrotron ring boost them to $350 \mathrm{MeV}$ for injection into the VEPP3 storage ring. The VEPP-3 storage ring stores $2 \mathrm{GeV}$ positrons and finally feeds the VEPP-4M collider where the $\mathrm{e}^{+} \mathrm{e}^{-}$center-of-mass energy is up to $12 \mathrm{GeV}$. A schematic layout of the VEPP-4 accelerator complex is shown in Fig. 23.

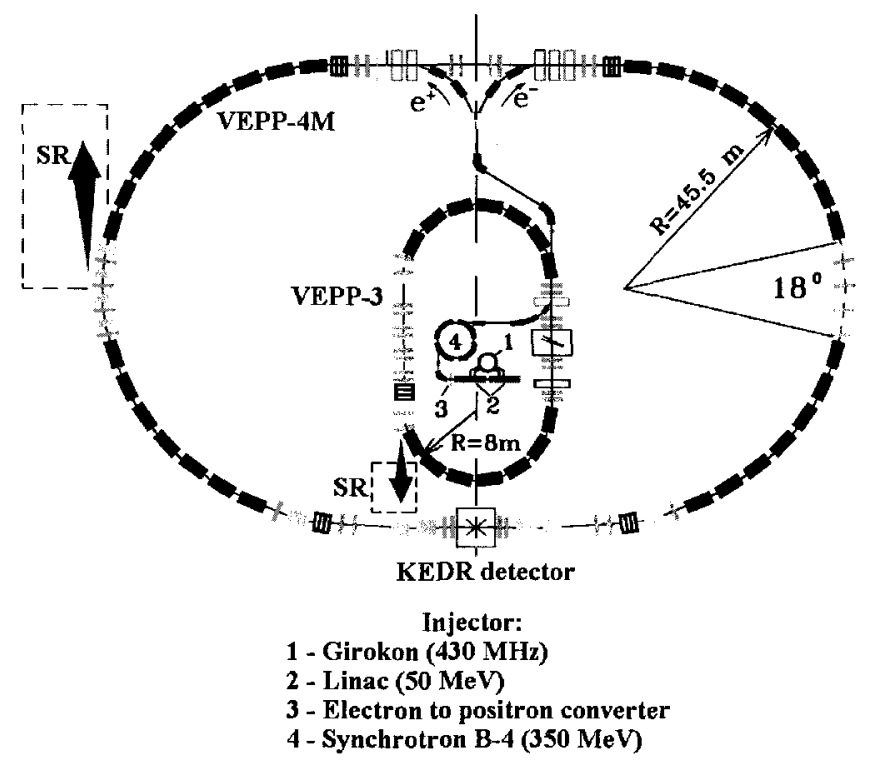

FIG. 23: The schematic layout of the VEPP-4 accelerator-ring complex. 1) Girokon (430 MHz), 2) The injector linac with a maximal beam energy of $50 \mathrm{MeV}, 3$ ) Positron conversion assembly, 4) Synchrotron booster with $350 \mathrm{MeV}$ energy [40].

To create positrons, electrons are accelerated to $270 \mathrm{MeV}$ in the first linac then hit a conversion target and produce $\mathrm{e}^{+} \mathrm{e}^{-}$pairs. The produced number of positrons per pulse is $5 \times 10^{8}$ at $70 \mathrm{MeV}$. It is expected that at a frequency of $50 \mathrm{~Hz}$ up to $10^{10}$ positrons per second will be obtained [41]. The electrons or positrons are injected from the injector with $1 \mathrm{~Hz}$ rate. The maximum stored $\mathrm{e}^{-}$beam is 500 $\mathrm{mA}$ with a storage rate of $2-5 \mathrm{~mA} / \mathrm{min}$ where the maximum stored $\mathrm{e}^{+}$current is $50 \mathrm{~mA}$ with a storage rate of $0.04 \mathrm{~mA} / \mathrm{min}$. In four minutes, up to $160 \mathrm{~mA} \mathrm{e}$ e $^{-}$ current can be accelerated to $2 \mathrm{GeV}$ in VEPP-3 storage ring. What is claimed to 
be unique about the VEPP-4 facility is, it carries one of the best (claimed to be a world record [42]) absolute particle energy calibration of $10^{-6}$. This is achieved using the resonant depolarization technique. This particle energy calibration creates the possibility of measuring the masses of elementary particles with extremely high precision. Now, the VEPP-4M electron-positron collider is operating for high-energy physics experiments in the $1.5-2.0 \mathrm{GeV}$ energy range.

\section{II.2.5 International Linear Collider (ILC)}

The future International Linear Collider (ILC) will require of order $10^{14} \mathrm{e}^{+} / \mathrm{s}$ to reach the desired luminosity requirements. For this current, a baseline design has been studied by using an undulator based positron source $[43,44]$, which is illustrated in Fig. 24. A collimated beam of photons produced from the helical undulator is impinging on a conversion target creates positrons, where the produced positrons can then be captured, accelerated and injected into a damping ring. The intense photon beam from the helical undulator will show the same bunch characteristics as in the main ILC electron beam of 2820 bunches of 1 ps duration with $308 \mathrm{~ns}$ between bunches at a $5 \mathrm{~Hz}$ rate. The required energy for the photons is at least 10 $\mathrm{MeV}$, where beyond $10 \mathrm{MeV}$ pair creation dominates over photo-electric and compton interactions. The energy of the radiated photons from an undulator is given with the following expression [45]:

$$
E_{p h}[e V]=9.5 \frac{n E[G e V]^{2}}{\lambda_{u}[m](1+0.5 K)}
$$

where $n$ is the harmonic number, $E$ is the electron beam energy in $\mathrm{GeV}, \lambda_{u}$ is undulator periodic length in meters and $K$ is the undulator strength parameter defined as:

$$
K=93.4 B_{0}[T] \lambda_{u}[m]
$$

With $\mathrm{B}_{0}=1 \mathrm{~T}$, harmonic number $n=1$ and $100 \mathrm{~m}$ length undulator with a period of $\lambda_{u}=0.01 \mathrm{~m}$, the electron beam must be more than 130 (170) $\mathrm{GeV}$ to get this required $\sim 10(20) \mathrm{MeV}$ photon energy $[43,44]$. The photon beam creating these positrons will be incident on the rim of a titanium alloy composed of $90 \%$ titanium with a composition of $\mathrm{Ti}-6 \% \mathrm{Al}-4 \% \mathrm{~V}$ target wheel, 0.4 radiation lengths thick (14 $\mathrm{mm}$ ) with $2 \mathrm{~m}$ diameter. The schematical drawing of the proposed target is shown in Fig. 25. The target will be positioned at least $500 \mathrm{~m}$ downstream of the center of the 
undulator, giving a photon beam spot with an r.m.s radius of at least $1-2 \mathrm{~mm}$. The positron source community is pursuing several alternative technologies to develop a target capable of long-term operation in the intense photon beam. One alternative is a liquid jet target [46] is shown in Fig. 26. In Chapter V, these target options considered in much broader details.

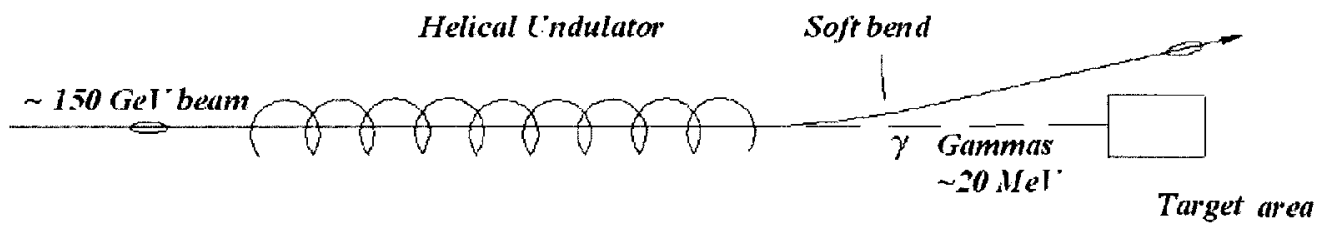

FIG. 24: The helical undulator used to produce photons to create positrons [47].

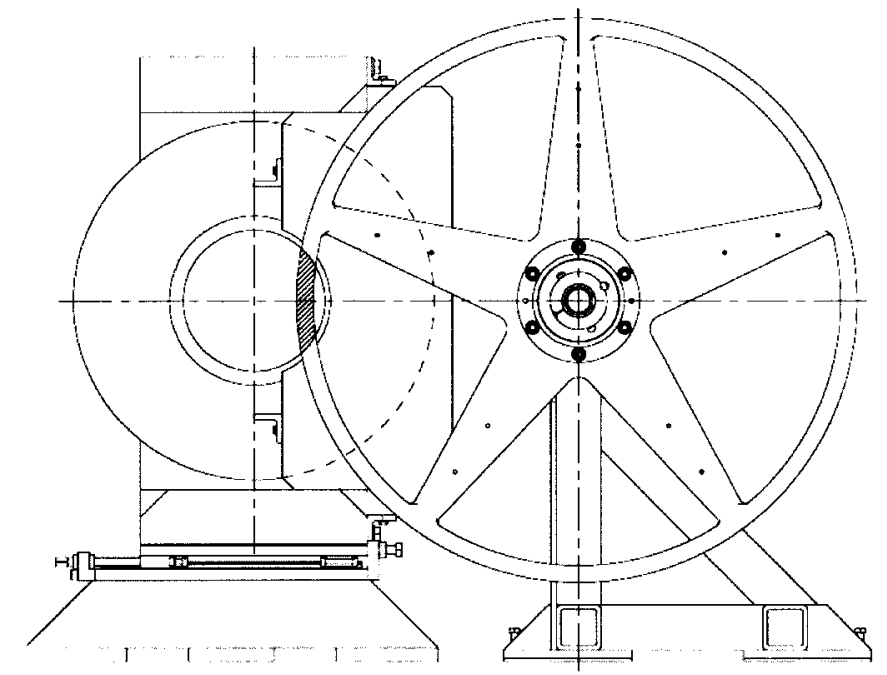

FIG. 25: Cross section of the proposed ILC rotating positron converter target assembly [48]. 


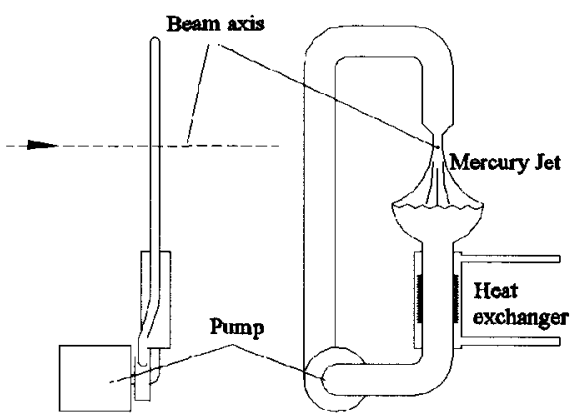

FIG. 26: An alternative liquid jet target for ILC positron source [47].

\section{II.2.6 Conclusion}

There are various positron sources at different facilities, which for different purposes the sources have different features. Some of them use positrons for low energy physics; such as material defects or angular correlated annihilation experiments. In high energy and nuclear physics experiments utilization of positrons have contributed major improvements. We also have seen that radioactive sources will not be suitable for the CEBAF positron source because of the current limitation. The SLAC positron source reached one of the highest beam currents $\sim 10^{12} \mathrm{e}^{+} / \mathrm{s}$, while future projects such as ILC investigates currents a factor of hundred more $\sim 10^{14}$ polarized $\mathrm{e}^{+} / \mathrm{s}$. All of these institutions use a pulsed time structure with high energy/high current incoming electron beam and almost always accompanied by a damping ring to reduce the large positron emittance by synchrotron radiation to achieve the desired luminosity requirements. As CEBAF operates at a CW mode $(1497 \mathrm{MHz})$ with all the buckets filled, the positrons injected into the ring will not be able to be damped before they leave, nor new positron injection will be possible. A damping ring, within a low cost project, is currently not feasible with the estimated damping time and energy range. A summary table of the positron sources is shown in Table 9. It can be seen from this table that some of the sources are cited as having ampere currents. But in fact, these currents are the beam current values in their damping rings accumulated over time, or the peak current values. Since for most experiments the final current is important, it has not been taken as a priority to measure the positron efficiency at the target or at the end of the capture system for most institutions. For obsolete machines and more detailed ring properties interested readers can find in this Ref. [49]. 
TABLE 9: Summary table for some of the mentioned positron sources.

\begin{tabular}{|c|c|c|c|c|c|c|c|c|}
\hline \multirow{2}{*}{ Facilities } & \multicolumn{4}{|c|}{ Driving $\mathrm{e}^{-}$Beam } & \multicolumn{2}{|c|}{$\mathrm{e}^{+}$Source } & \multirow{2}{*}{\multicolumn{2}{|c|}{$\begin{array}{c}\varepsilon\left(\mathrm{e}^{+}\right) \\
(\mathrm{mm} \cdot \mathrm{mrad})\end{array}$}} \\
\hline & $\mathrm{E}(\mathrm{GeV})$ & $\mathrm{N}\left(\mathrm{e}^{-}\right)$ & $\omega(\mathrm{Hz})$ & $\operatorname{Size}(\mathrm{mm})$ & Target $(\mathrm{mm})$ & Matching & & \\
\hline SLAC & 33 & $4 \times 10^{10}$ & 120 & 2.5 & $\mathrm{~W}-\mathrm{Ta}(24)$ & AMD & $2 \times 10^{12}$ & $25($ at $200 \mathrm{MeV})$ \\
\hline BEPCII & 0.24 & $4.5 \times 10^{18}$ & 50 & $1-3$ & $\mathrm{~W}(8 \mathrm{~mm})$ & AMD & $10^{16}$ & $1.6(1.89 \mathrm{GeV})$ \\
\hline CESR & $\overline{0.2}$ & $2 \times 10^{11}$ & 50 & 2.5 & W-Re & QWT & $10^{10}$ & - \\
\hline KEK [50] & 0.25 & $6 \times 10^{19}$ & - & - & $\mathrm{Ta}$ & QWT & $6 \times 10^{17}$ & $2($ at $1 \mathrm{GeV})$ \\
\hline VEPP4 & 0.27 & $1.6 \times 10^{11}$ & 1 & - & $\bar{W}$ & AMD & $5 \times 10^{8}$ & - \\
\hline ILC & 130 & $2.8 \times 10^{13}$ & 5 & 0.75 & W76Re24 & AMD & $10^{14}$ & - \\
\hline CEBAF & 0.126 & $6 \times 10^{16}$ & $1.497 \times 10^{9}$ & 0.1 & $\mathrm{~W}$ & QWT & $10^{12}$ & $1($ at $120 \mathrm{MeV})$ \\
\hline
\end{tabular}




\section{CHAPTER III}

\section{ADMITTANCE MEASUREMENT AT CEBAF}

In Chapter I, the properties of the CEBAF electron beam were introduced. As it was given in Table 2, the normalized emittance of the electron is $\varepsilon_{N}=10^{-6} \mathrm{~m} \cdot \mathrm{rad}$, and the fractional energy spread at the full energy of $6 \mathrm{GeV}$ is $\delta<10^{-4}$. This implies a spread at the injection energy of $65 \mathrm{MeV}$ of $\delta<10^{-2}$. The conventional positron production method is the result of pair conversion of photons created via bremsstrahlung of electrons in a high- $Z$ target. At the CEBAF injection energies, the multiple scattering in a thick target $(1-3 \mathrm{~mm} \mathrm{~W}$ or $\mathrm{Pb})$ is of the order of $\sigma(\theta) \sim 200$ mrad and the energy spread is too large. Even with a spot size as small as 100 microns, this implies a positron transverse emittance a few thousand times larger than the CEBAF electron beam. For this reason, we started a study to determine the largest transverse and longitudinal phase space that can be transported in CEBAF.

\section{III.1 TRANSVERSE ADMITTANCE}

The Admittance (Acceptance), in the accelerator terminology [8]; is a property of the accelerator hardware and the whole lattice. It is the maximum transverse and longitudinal phase space of the beam that can be transported throughout the accelerator.

As the beam has a finite size, it is limited with the accelerator beam pipe or other apertures. The equation describing relation with the geometrical transverse admittance, $A_{T}$, aperture width and the $\beta(s)$ is given by;

$$
A_{T}=\left(\frac{d^{2}}{\beta}\right)
$$

where $d$ is the half aperture of the beam transport pipe and $\beta$ is the twiss parameter as described in Eq. (38). The admittance ellipse is illustrated in Fig. 27. In most systems such as storage rings, it is required to "stay clear away" from the beam pipe walls in transverse size by $7 \sigma$ as the large betatron oscillations may cause problems after many revolutions [5]. For the CEBAF positron source we can relax stay clear

away requirement as low as $3 \sigma$. But we need to quickly transport the beam to an accelerating unit to take advantage of adiabatic damping from the acceleration 


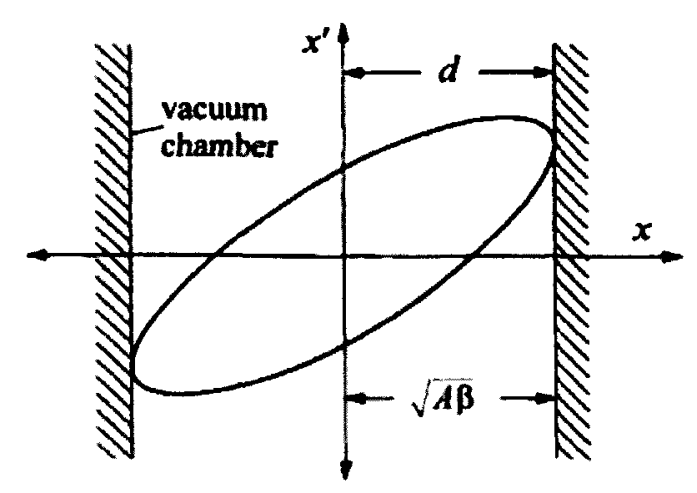

FIG. 27: The admittance ellipse extending to the walls of the beam pipe [5].

which will reduce the geometrical emittance by a factor $1 / \gamma$, where $\gamma$ is the relativistic Lorentz factor $E / m$.

As the positrons come out of the tungsten production target, they have a very large emittance, thus it must be known how many positrons can be transported safely in the machine. An admittance measurement in the injector region of the CEBAF was performed to get a rough estimation of the admittance in this area. The measurement used two corrector magnets (kicker magnets), namely MATOR05H (or $05 \mathrm{~V}$ ) and MATOR09H (or $09 \mathrm{~V}$ ) kicker magnets where $(\mathrm{H}$ ) denotes horizontal and (V) denotes vertical [51]. These air core magnets are used for orbit correction of the beam.

In Fig. 28, it is shown at which location the CEBAF admittance measurement was done. In Fig. 29, the actual schematic of the region is shown. The admittance measurement was made at the region between Beam Position Monitors (BPM) IPM0L09 and IPM0L10; where IPM0R06 was used as the current monitor for the first test. For the second test, measurement was performed at the same region, with the addition of downstream BPMs ; IPM0R07, IPM1L02 and IPM1A39 as beam current monitors.

In order to create a transverse phase space, two different locations are required to introduce deviation angle from the reference orbit. MAT0R05H(V) magnet was used to kick the beam in $\mathrm{H}(\mathrm{V})$ direction, this transverse displacement was detected at the downstream Beam Position Monitor(BPM), IPM0L09, and then MAT0R09H(V) was used to introduce a deviation in angle with respect to the reference frame, where this 


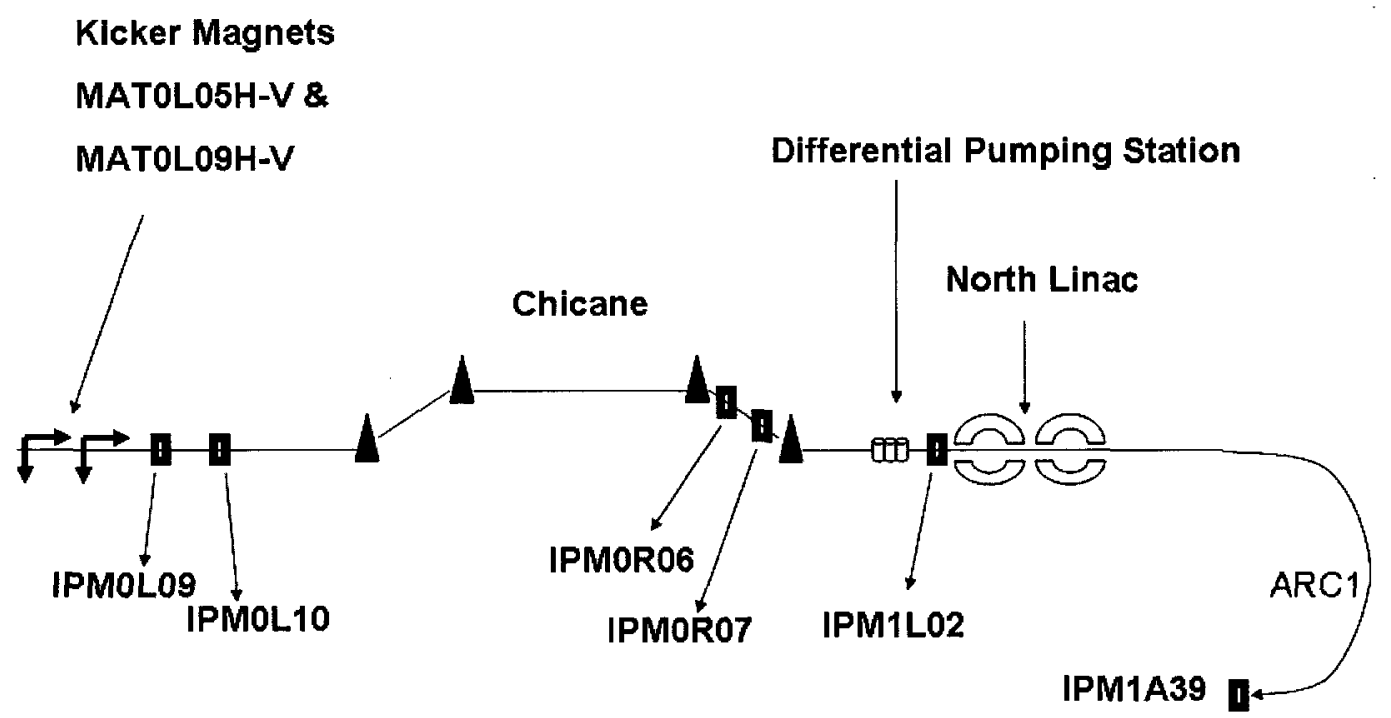

FIG. 28: Simple schematic of the location of the CEBAF admittance test.

deviation was recorded as;

$$
\theta \approx \tan \theta=\frac{X(I P M 0 L 10)-X(I P M 0 L 09)}{611 \mathrm{~cm}}
$$

A complete scan of the region was done separately in each direction to get an uncoupled beam emittance by scanning the corrector magnets from $\mathrm{B}(\min )=-300$ Gauss to $\mathbf{B}(\max )=300$ Gauss. The scan was automated by a program which interacts with the corrector magnets and the BPMs. Nominal BPM wire sums were recorded initially for determining the full beam transport as a reference value. During the scan, the beam was considered as "lost" when wire sum readouts from the BPMs were less than $10 \%$ of the nominal values.

The first transverse admittance data are shown in Fig. 30. The data plotted in this figure shows the transverse phase-space area, which only IPM0R06 (in the injector at a prior location of the North Linac) was used as the current monitor. The second admittance measurement data, which are shown in Fig. 31, propagated the beam all the way around the first arc, ARC1. There are less data in this scan (no excursions in $x<-2 \mathrm{~mm}$ ), but the admittance function otherwise has a similar shape and area as the first scan. It is seen that if the beam goes through the injector chicane, it goes through the machine until the end of the ARC1. The BPM wire sums registered approximately the same fractional currents for both IPM0R07 and 


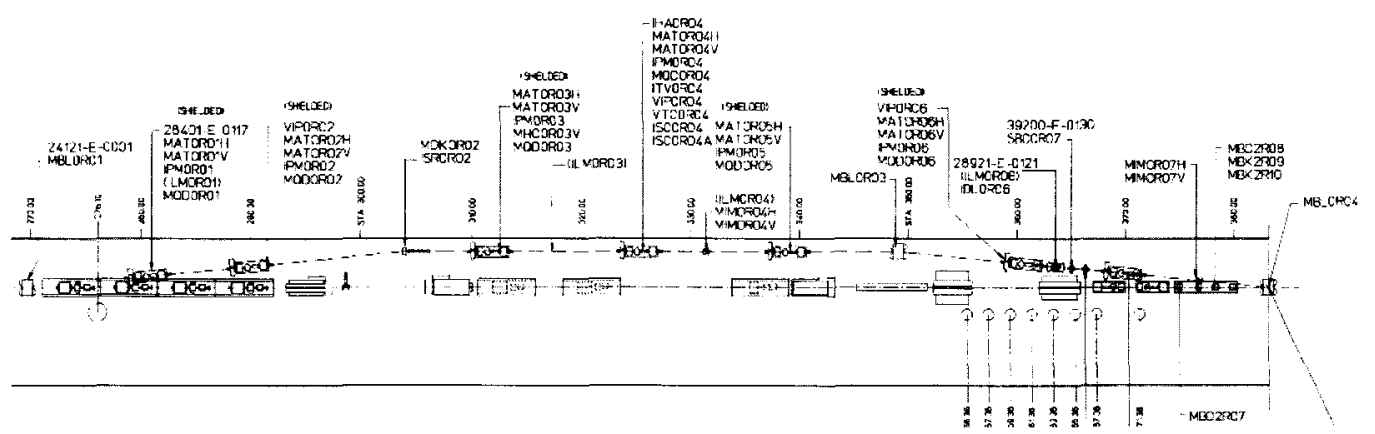

FIG. 29: Actual scaled schematic of the location of the CEBAF admittance test. This is the chicane section of the injector where the beam is injected into the North Linac. The names label the instruments used in the beam line. All elements are not shown in this partial schematic [52].

IPM1A39. Thus particles that are transported through the injection chicane are transported all the way around the arc. Vertical data for the second set (IPM1A39) were not complete, and are not considered here. The measured admittance area in horizontal and vertical are $\sim 10$ and $\sim 5 \mathrm{~mm} \cdot \mathrm{mrad}$ respectively (or $\sim \pi 3.2$ and $\sim \pi 1.6$ $\mathrm{mm} \cdot \mathrm{mrad})$. It is clearly seen from the figure that these phase space areas are not type of elliptical areas but more like a rhombus shape hard cuts. These areas can be taken as rough estimates about the maximum positron phase space area that can be used in the CEBAF positron source. When we analyze this result it has come to our attention that the results were lower than our calculated figures. So we can speculate that the injector chicane could be a limiting structure or another possibility that the "Differential Pumping"(DP) cans, which will be discussed later in Chapter IV, were limiting structures for the admittance measurements.

There are quadrupoles or corrector magnets about every 2-3 meters to control the beam as close as to the reference orbit, but let us think that we have a $50 \mathrm{~m}$ drift space where the beam pipe diameter is $20 \mathrm{~mm}$ at the narrowest location. In this drift space the beam size will grow very slowly due to the very small angle of the electron beam $\sigma_{x^{\prime}} \sim 10^{-4} \mathrm{rad}$ and will eventually hit the walls. But the beam will make through this drift space giving us an admittance value of $A_{T}=$ Beam Pipe Radius $\times$ Drift angle $=10 \mathrm{~mm} \times \arctan \left(\frac{10 \mathrm{~mm}}{50 \times 10^{3} \mathrm{~mm}}\right) \sim 500 \mathrm{~mm}$.mrad. Of course this is a naive estimation that omits the quads and correctors. When we kick the beam, it simply goes through the magnets and at these locations where the $\beta$ 


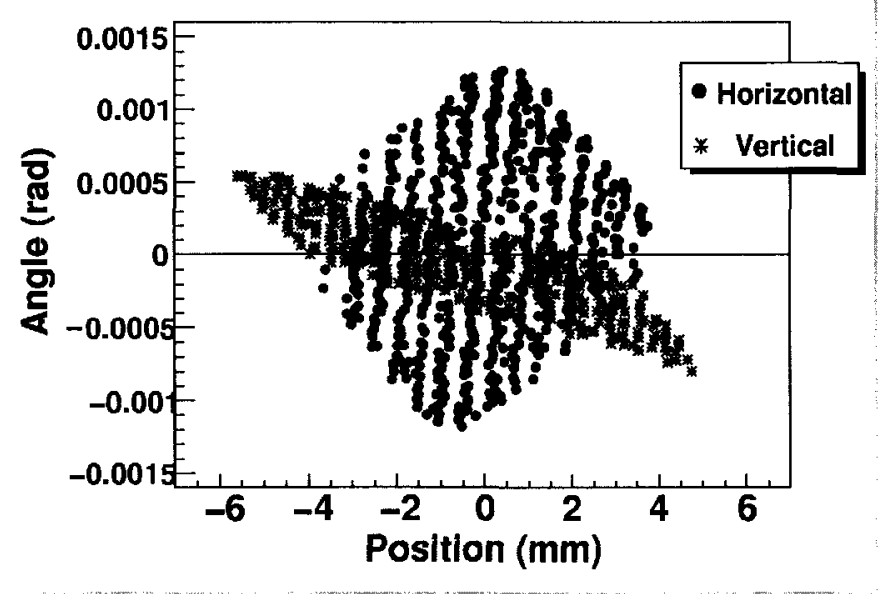

FIG. 30: The $\mathrm{H}$ and $\mathrm{V}$ Admittance phase space area in the injector where the IPM0R06 was used as a current monitor.

function is already too large, the beam scrapes the walls. It is worth to note that the design orbit for the CEBAF injector calls for maximum $\beta_{\max } \sim 30-50 \mathrm{~m}$ at certain locations depending on the tune of the magnets. Considering the narrowest place in the beamline where radius $d=10 \mathrm{~mm}$ so from Eq. (71);

$$
A_{T}=\frac{d^{2}}{\beta_{\max }}=\frac{10^{2} \mathrm{~mm}^{2}}{50 \times 10^{3} \mathrm{~mm} \cdot \mathrm{rad}^{-1}}=2 \mathrm{~mm} \cdot \mathrm{mrad}
$$

gives estimated admittance of the injector between $\sim 2-4 \mathrm{~mm} . \mathrm{mrad}$, in fact this is mainly due to the very large $\beta$ design in the injector area. This estimation is in agreement with our admittance measurement. There are many factors effecting the admittance of the machine, such as the tune of the magnets. Apparently, this $\beta$ functions were eased a little bit in order to tune the beam correctly in the injector. Although it not possible to expect that the geometrical admittance is as high as 500 $\mathrm{mm} . \mathrm{mrad}$ as calculated with only the presence of a drift space, but indeed we can be very optimistic that with a reasonable improved design $\beta$ functions, the CEBAF admittance can be as high as 20 - $30 \mathrm{~mm}$.mrad. This is a reasonable range since the average $\beta$ value in the injector is $\sim 15 \mathrm{~m}$, which is a factor of three lower than $\beta_{\max }$ and the beam pipe aperture is $\sim 35 \mathrm{~mm}$. 


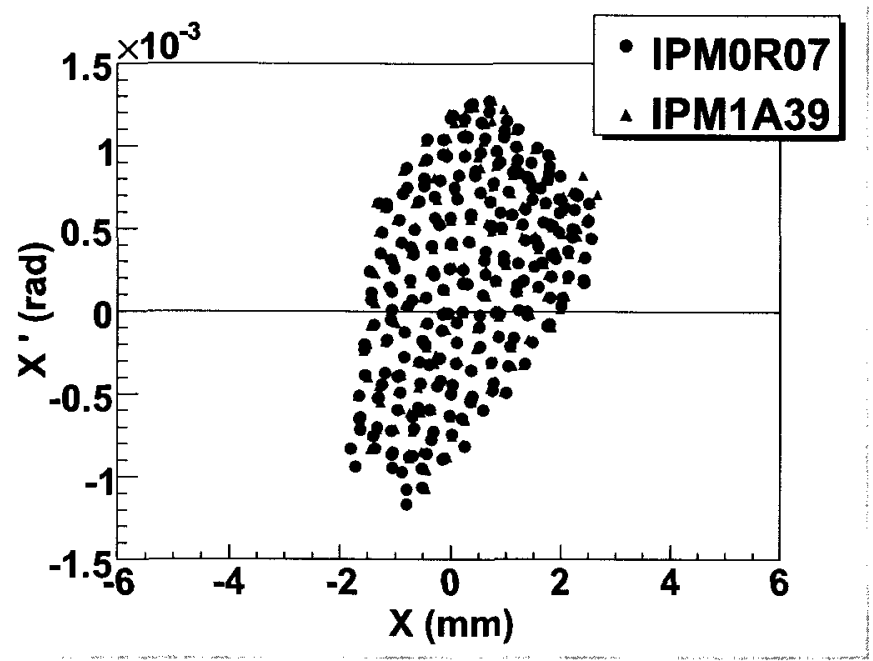

FIG. 31: Second set of horizontal data where IPM0R07 and IPM1A39 were used as current monitors.

\section{III.2 LONGITUDINAL ADMITTANCE}

In longitudinal phase space (E-t), the beam is limited due to the certain energy spread requirements of the experiments and also because of the limitation on the geometry of the ARCs. Since the beam is accelerated, it is also limited with another parameter, which is time structure of the positrons for on crest acceleration to get the full power from the RF. The superconducting cavities (SRF) in CEBAF operate at $1497 \mathrm{MHz}$ frequency. As a starting estimation, if the energy spread of the positrons at the $\mathrm{ARC} 1$ is required to be $\delta \leq 10^{-3}$, then the time spread must follow;

$$
\begin{array}{r}
E=E_{0} \cos (\omega t) \\
\omega=2 \pi f \\
\cos (\omega t)>0.999 \\
|\omega t|<0.045 \mathrm{rad} \\
t<\frac{|\omega t|}{2 \pi f}=4.7 \mathrm{ps}
\end{array}
$$

where $E$ is the energy gain in the accelerator and $w t$ is the synchronous phase, with $t$ being the time (off crest) of a particle. 
To measure the maximum tolerated energy spread in the ARC1, a measurement was conducted at ARC1 during the first pass of the beam with a normal operational tune. Initially, the beam was set on the nominal energy with a fractional energy spread of $\delta=10^{-4}$ at $653.2 \mathrm{MeV} / \mathrm{c}$ momentum. Then with an automated script, the beam energy was increased slowly. The beam made through cleanly to the last BPM in the ARC1 which is IPM1A39. The snapshot from the script is shown in Fig. 32, which shows each Set and Measured RF cavity energy under the cavity section on the left; here $d p / p$ is set to $3 \times 10^{-3}$. In the middle of the snapshot the status of the BPM is shown. As it is seen in the figure, the BPM status of IPM1A39 is OK, meaning that the beam is still measured. In Fig. 33, the Beam Energy Status monitor snapshot is shown. The measured momentum and fractional energy spread are $654.9 \mathrm{MeV} / \mathrm{c}$ and $\delta \sim 2.7 \times 10^{-3}$ respectively. From the nominal beam momentum $653.2 \mathrm{MeV} / \mathrm{c}$, this spread is equal to $\Delta p=1.7 \mathrm{MeV} / \mathrm{c}$. This was the upper limit energy spread for the positive increase, after this step the BPM did not measure any beam signal. In Figs. 34 and 35, the snapshots for decrease from the nominal energy in the ARC1 are shown. In the negative side the beam energy is measured $651 \mathrm{MeV} / \mathrm{c}$ with $\delta \sim-3.3 \times 10^{-3}$ as a lower limit. From the nominal beam energy, this will result $\Delta p=-2.2 \mathrm{MeV} / \mathrm{c}$ deviation from the central momentum. By taking the average we can say that the ARC1 can tolerate $\sim 653 \pm 2 \mathrm{MeV} / \mathrm{c}$. We note that if the energy spread is $3 \times 10^{-3}$ at ARC1, then provided the condition of Eq. (75) is satisfied, the energy spread will be less than $10^{-3}$ after just two full passes in CEBAF. Thus we estimate the admittance at the injector (entrance to first pass in North LINAC) as $\approx \pm 10 \mathrm{ps} . \mathrm{MeV}$, for an admittance area of $\pi(20 \mathrm{ps} . \mathrm{MeV})$. 


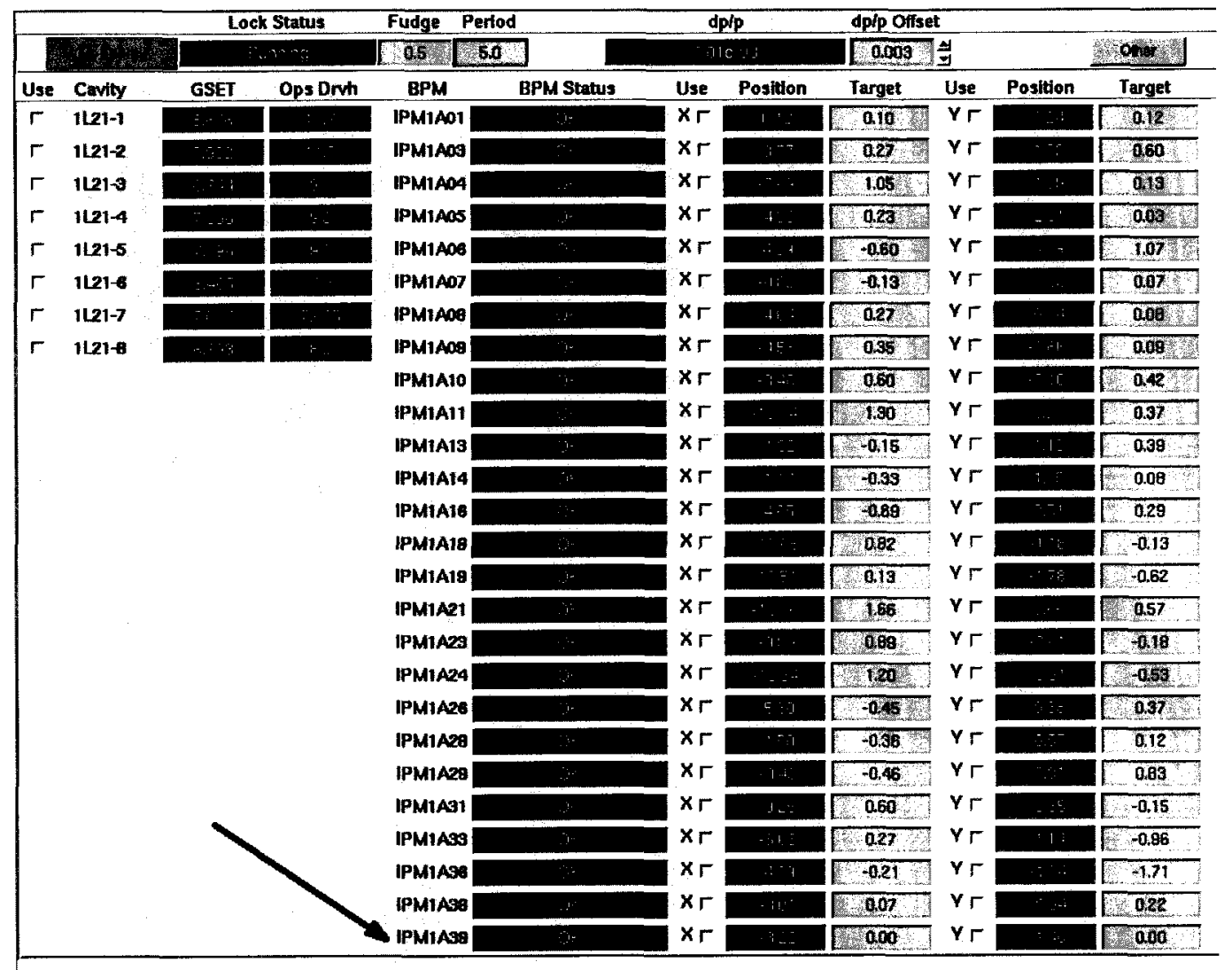

FIG. 32: The figure shows each set and measured RF cavity loads, and measured $x$ and $y$ positions of the electron beam at certain BPMs. In the script $\mathrm{dp} / \mathrm{p}$ is set to $3 \times 10^{-3}$. In the middle the BPM statuses are shown. IPM1A39 is the last BPM in the $\mathrm{ARC} 1$ and still registering signal at this energy offset. 


\begin{tabular}{|c|c|c|c|c|c|c|c|c|}
\hline & & & & $D_{1}$ & & 国 & a & II \\
\hline & offion & & & & & server hear & best & \\
\hline Master & off $\sqrt{10}$ & dp/p bpm & $\mathrm{dp} / \mathrm{p}$ corr & dp/p total & $\mathrm{MeV}$ & Target MeV & Status & Invalid \\
\hline DINJ & onf $\sqrt{10 n}$ & $-4.2996 \mathrm{e}-04$ & $7.9267 \mathrm{e}-03$ & $7.4968 \mathrm{e}-03$ & 66.7200 & 66.40 & DOSIBEAM & \\
\hline DARC1 & anl $\sqrt{\text { on }}$ & $2.7927 \mathrm{e}-03$ & $-5.9655 c-05$ & $2.7331 \mathrm{e}-03$ & 654.9306 & 656.60 & Beam Here & \\
\hline$\square$ ARC2 & ont $\sqrt{\text { on }}$ & $0.0000 e+00$ & $0.0000 \mathrm{e}+00$ & $0.0000 e+00$ & 1246.3748 & $1246.80^{\circ}$ & NO BEAM & \\
\hline DARC3 & off $\sqrt{\text { in }}$ & $0.0000 e+00$ & $0.0000 e+00$ & $0.0000 \mathrm{e}+00$ & 1835.1647 & 1837.00 & No Beam & \\
\hline D ARC4 & off $\sqrt{\text { क }}$ & $0.0000 e+00$ & $0.0000 e+00$ & $0.0000 \mathrm{e}+00$ & 2434.1260 & 2427.20 & NO BEAM & \\
\hline DARC5 & 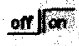 & $0.0000 e+00$ & $0.0000 \mathrm{e}+00$ & $0.0000 \mathrm{e}+00$ & 3021.6721 & 3017.40 & No Beam & \\
\hline D. ARC6 & onflion & $0.0000 e+00$ & $0.0000 e+00$ & $0.00000+00$ & 3607.4957 & 3607.60 & NO BEAM & \\
\hline D. ARC7 & off $\sqrt{\frac{10}{2}}$ & $0.0000 e+00$ & $0.0000 \mathrm{e}+00$ & $0.0000 e+00$ & 4200.6402 & 4197.30 & No Beam & \\
\hline$\square$ ARCB & off $\sqrt{\text { on }}$ & $0.0000 e+00$ & $0.0000 e+00$ & $0.0000 e+00$ & 4790.2734 & 4788.00 & NO BEAM & \\
\hline DARC9 & onf $\sqrt{[\mathrm{m}}$ & $0.0000 e+00$ & $0.0000 \mathrm{e}+00$ & $0.0000 e+00$ & 5378.9897 & 5378.20 & No Bean & \\
\hline HALLA & oft $\sqrt{\mathrm{cm}}$ & $0.0000 e+00$ & $0.0000 e+00$ & $0.0000 e+00$ & 2425.5181 & 2427.20 & NO BEAM & \\
\hline GHALLC & off $\sqrt{10}$ & $0.0000 e+00$ & $0.0000 e+00$ & $0.0000 e+00$ & 5971.7247 & 5968.40 & NO BEAM & \\
\hline
\end{tabular}

FIG. 33: The Beam Energy Status monitor snapshot is shown. The measured fractional energy spread is $\delta \sim 2.73 \times 10^{-3}$ measured at $655 \mathrm{MeV} / \mathrm{c}(\Delta p=+1.7 \mathrm{MeV} / \mathrm{c})$. This was the limit energy spread for the positive increase, after this the BPM did not measure any beam signal. 


\begin{tabular}{|c|c|c|c|c|c|c|c|c|c|c|c|}
\hline & & & Status & Fudge & & & & dplp Offse & & & \\
\hline & $M$ & & 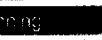 & $0.5]$ & & & $\rightarrow-1$ & 0.009 & & & 2 oner \\
\hline Use & Cavity & GSET & Ops Drh & BPM & BPM Status & Use & Position & Target & Use & Position & Target \\
\hline$r$ & $1621-1$ & 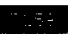 & & IPMIAOI & & $x r$ & $\therefore$ & 0.10 & Yr & & 0.12 \\
\hline$r$ & $1121-2$ & .. & & IPM1A0s & & $x r$ & $\ldots$ & 0.27 & vr & & 0.60 \\
\hline$r$ & $1121-9$ & - & & IPM1AD4 & & $x$ & 6 & 1.05 & yr & $\because$ & 0.13 \\
\hline$r$ & $1121-4$ & & & IPM1AOS & & $x \Gamma$ & - & 0.23 & Qr $_{r}$ & & $\longdiv { 0 . 0 3 }$ \\
\hline$r$ & 1L21-5 & & & IPMIAOA & & $x \Gamma$ & 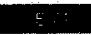 & -0.60 & Vr & & 1867 \\
\hline$r$ & $1121-8$ & & & IPMIA07 & & $x r$ & 2 & -0.13 & Y r & $\because$ & 0.07 \\
\hline$r$ & 1L21-7 & -1 & & IPMIAOB & & $x r$ & $\because$ & 0.27 & Yг & & 0.08 \\
\hline$r$ & 11218 & & & IPM1AOC & & $x=$ & & 0.35 & Yr & & 0.09 \\
\hline & & & & IPM1A10 & & $\times r$ & $=$ & 0.60 & Yr & & 0.42: \\
\hline & & & & IPM1A11 & & $x r$ & $\ldots$ & 1.30 & vr & $\therefore$ & . 697 \\
\hline & & & & IPMIAIa & & $x \Gamma$ & & -0.15 & $\mathrm{Yr}_{\mathrm{r}}$ & & 068 \\
\hline & & & & IPMIAIA & & $x \Gamma$ & & -0.39 & Yr & & 0.00 \\
\hline & & & & IPMIAIE & & $x$ & 4 & -0.69 & vr & $1:$ & 0.28 \\
\hline & & & & IPM1A1E & & $x r$ & $\rightarrow$ & 0.82 & Yr & & 0.13 \\
\hline & & & & IPM1A19 & & $x r$ & 5 & 0.13 & $\mathrm{Yr}_{\mathrm{r}}$ & Fis & -0.62 \\
\hline & & & & IPMTAZI & & $x \Gamma$ & & 156 & Yr & & 067 \\
\hline & & & & IPMIAZS & & $x \Gamma$ & & 0.69 & Yr & 8 & 0.18 \\
\hline & & & & IPMIAZ4 & & $x r$ & & 120 & $\mathbf{Y r}_{\mathrm{r}}$ & & -0.53 \\
\hline & & & & IPMIAZE & & $x$ & & 0045 & Yr & & 0.37 \\
\hline & & & & IPM1AZ: & & $x r$ & & -0.36 & $v_{r}$ & $7=$ & 0.12 \\
\hline & & & & IPM1A28 & & $x \Gamma$ & 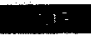 & -0.46 & Yr & $E$ & 683 \\
\hline & & & & IPMIA9I & & $x$ & $\therefore$ & 0.60 & $v_{r}$ & $\therefore=$ & 075 \\
\hline & & & & IPMIAGS & & $x$ & 1 & 0.27 & vr & it & -0.86 \\
\hline & & & & IPMIA9E & & $x$ & 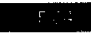 & -0.21 & vr & $\Delta$ & -1.71 \\
\hline & & & & IPM1ASE & & $\times r$ & 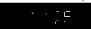 & 0.07 & $\mathrm{Yr}$ & 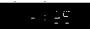 & D.22 \\
\hline & & & & IPM1As9 & & $x \Gamma$ & & 0.00 & Yг & $1=$ & 000 \\
\hline
\end{tabular}

FIG. 34: The figure shows each set and measured RF cavity loads, and measured $x$ and $y$ positions of the electron beam at certain BPMs. In the script $\mathrm{dp} / \mathrm{p}$ is set to $-3 \times 10^{-3}$. In the middle the BPM statuses are shown. IPM1A39 is the last BPM in the ARC1 and still registering signal at this energy offset. 


\begin{tabular}{|c|c|c|c|c|c|c|c|c|}
\hline \multirow{2}{*}{\multicolumn{5}{|c|}{ offion }} & \multirow{2}{*}{\multicolumn{4}{|c|}{ server heartbeat }} \\
\hline & & & & & & & & \\
\hline Master & off $\sqrt{\text { on }}$ & dp/p bpm & dp/p corr & dp/p total & $\mathrm{MeV}$ & Target MeV & Status & \\
\hline 명 INJ & off $\sqrt{\text { on }}$ & $-4.4642 \mathrm{e}-04$ & $7.9267 e-03$ & $7.4803 \mathrm{e}-03$ & 66.7189 & \multicolumn{3}{|c|}{ 66.40 POSIBEAM } \\
\hline D ARC1 & off $\sqrt{\infty}$ & $-3.2470 c-03$ & $-5.9655 e-05$ & $-3.3066 \mathrm{e}-03$ & 650.9858 & 656.60 & Beam Here & \\
\hline 드 ARC2 & off $\sqrt{\infty}$ & $0.0000 \mathrm{e}+00$ & $0.0000 \mathrm{e}+00$ & $0.0000 e+00$ & 1246.3748 & 1246.80 & NO BEAM & \\
\hline D) ARC3 & off $\sqrt{\text { on }}$ & $0.0000 e+00$ & $0.0000 e+00$ & $0.0000 e+00$ & 1835.1647 & 1837.00 & No Beam & \\
\hline D ARC4 & off $\sqrt{10 n}$ & $0.0000 \mathrm{e}+00$ & $0.0000 e+00$ & $0.0000 \mathrm{e}+00$ & 2434.1260 & 2427.20 & NO BEAM & \\
\hline$\square$ ARC5 & off $\sqrt{\infty}$ & $0.0000 e+00$ & $0.0000 e+00$ & $0.0000 \mathrm{e}+00$ & 3021.6721 & 3017.40 & No Beam & \\
\hline D ARC6 & onf $\sqrt{\infty n}$ & $0.0000 e+00$ & $0.0000 e+00$ & $0.0000 \mathrm{e}+00$ & 3607.4957 & 3607.60 & NO BEAM & \\
\hline D ARC7 & off $\sqrt{\text { on }}$ & $0.0000 e+00$ & $0.0000 e+00$ & $0.0000 \mathrm{e}+00$ & 4200.6402 & 4197.80 & No Beam & \\
\hline D ARC8 & off $\sqrt{\text { on }}$ & $0.0000 \mathrm{e}+00$ & $0.0000 e+00$ & $0.0000 \mathrm{e}+00$ & 4790.2734 & 4788.00 & NO BEAM & \\
\hline 다 ARC9 & off $\sqrt{\text { on }}$ & $0.0000 \mathrm{e}+00$ & $0.0000 e+00$ & $0.0000 e+00$ & 5378.9897 & 5378.20 & No Beam & \\
\hline DHALLA & off $\sqrt{\infty}$ & $0.0000 e+00$ & $0.0000 e+00$ & $0.0000 e+00$ & 2425.5181 & 2427.20 & NO BEAM & \\
\hline 9 HALLC & onf $\sqrt{\infty}$ & $0.0000 e+00$ & $0.0000 \mathrm{e}+00$ & $0.0000 \mathrm{e}+00$ & 5971.7247 & 5968.40 & NO BEAM & \\
\hline
\end{tabular}

FIG. 35: The Beam Energy Status monitor snapshot is shown. The measured fractional energy spread is $\delta \sim-3.3 \times 10^{-3}$ measured at $651 \mathrm{MeV} / \mathrm{c}(\Delta p=-2.2 \mathrm{MeV} / \mathrm{c})$. This was the limit energy spread for the negative increase, after this the BPM did not measure any beam signal. 


\section{CHAPTER IV}

\section{DESIGN OPTIONS FOR A POSITRON SOURCE}

In Chapter II, we reviewed some of the most prominent and high current laboratories' methods for the way they create the positrons, how these positrons are captured, and how their large angular and energy spread are handled so that they are more like a beam rather than a spray of particles. Some of these institutions use a primary electron beam impinging on a target; some (e.g ILC) propose to use photons created via an undulator and then hit this photon beam on a target to create positrons. For front end capture optics almost all of the designs used different types of solenoid magnets followed immediately by an accelerating unit. This accelerating unit is generally warm RF cavity bathed within another solenoid. Almost all positron sources use a damping ring to reduce the large phase space of the positron beam.

In this chapter, we present the solutions that are suitable for CEBAF requirements. The following outline gives an introductory for the main considerations and challenges of a positron source and methods to counter these challenges.

- Obtain positrons by the most efficient way.

As it is introduced in Chapter II, some future projects refer to photons directly hitting the converter target as this is an efficient way of creating electronpositron pairs, but this requires a long undulator with $\geq 150 \mathrm{GeV}$ electron beam. For CEBAF we recommend:

$\checkmark \quad$ Use the conventional method to obtain the pair production via bremsstrahlung photons.

- Capture as much as positrons immediately after the $\mathrm{e}^{+}$converter target. Useful current in the interest for all Halls is $\geq 100 \mathrm{nA}$

Almost all of the sources include a different type of solenoid, with a mixture of short and long solenoids. Solenoids are used to focus the beam transversely at the front end. For CEBAF we recommend a short solenoid:

$\checkmark$ Quarter Wave Transformer (QWT) solenoid.

- Separate positron beam from electrons and other radiation. 
All sources have used a single dipole or a set of dipoles to achieve this. For CEBAF we recommend three different methods:

$\checkmark$ 1.Design : Position a Combined Function Magnet (CFM) Triplet after the QWT solenoid. In CFM configuration, all the primary and secondary particles are separated immediately. CFM magnets have both dipole and quadrupole fields.

$\checkmark$ 2.Design : Position a Quadrupole Triplet and two normal(or sector) dipoles after the QWT, where dipoles separate positrons from electrons at a later stage.

$\checkmark$ 3.Design : Position a Quadrupole Triplet and a microtron dipole set after the QWT, where microtron dipoles separate positrons from electrons at a later stage.

- Achromatic lattice.

$\checkmark$ All the design options must be achromatic lattice, which the dispersion function and its derivative are zero at the end of the dipoles. In addition to that, microtron dipoles make the lattice achromatic and isochronous.

- Require high quality beam in transverse and longitudinal emittance.

Every pulsed source has used a small or a large damping ring to damp the $6 \mathrm{D}$ emittance of the positron beam. As mentioned through the text, this is not a viable option currently, so we recommend:

$\checkmark$ Use quarter (1/4) and full SRF cryomodules as closely as possible to the mentioned positron capture area and take advantage of the adiabatic damping to reduce the beam emittance.

In the next section the efficiency (created positron per incoming electron) and target thickness optimizations are introduced. The design parameters of these three methods are introduced by using the $6 \mathrm{D}$ phase space characteristics of this optimized positron output. 


\section{IV.1 OPTIMIZATION}

Since there is no damping ring, we need to get the maximum efficiency from the created positrons as every positron is important to us. The basic motivation behind this optimization process is; creating the maximum number of positrons from an optimized target thickness balanced with the limits of deposited power in this target. Another important parameter is that the emittance of the emitted positrons can be manipulated by changing the incoming electron beam spot size. The optimization of the converter target thickness study was done via simulation programs. To find the maximum number of positrons within the measured admittance area, the brightness of these positrons must be considered.

When the $12 \mathrm{GeV}$ upgrade is completed at the CEBAF, the injection beam energy at the 1st pass of North Linac (NL) will be around $126 \mathrm{MeV}$. The current injection beam energy is $65 \mathrm{MeV}$ at the same injection location. As a basis for this chapter the electron beam energy $126 \mathrm{MeV}$ is used to simulate positron production on a tungsten target. The optimization of the energy selection of positrons, target thickness, brightness of positrons, incoming electron beam size, deposited beam power in the target are presented. The peak values of the momentum distributions for different positron converter thickness do not change drastically, but the positron yield (raw number of positrons produced from the pair production conversion process) increases with the increasing target thickness.

In the simulation process, CEBAF quality electron beam parameters as shown in Table 10 are used for positron creation.

TABLE 10: The electron beam parameters used in the simulation process.

\begin{tabular}{llll}
\hline \hline Momentum & $P\left(e^{-}\right)$ & 126.0 & $\mathrm{MeV}$ \\
Fractional Spread in Momentum & $\delta$ & $10^{-4}$ & \\
Transverse Beam Size & $\sigma_{x y}$ & 100.0 & $\mu \mathrm{m}$ \\
Normalized Transverse Emittance & $\varepsilon_{x y}$ & $10^{-6}$ & $\mathrm{~m} \cdot \mathrm{rad}$ \\
Time Spread & $\sigma_{t}$ & 200.0 & $\mathrm{fs}$ \\
\hline \hline
\end{tabular}

The total momentum distribution plot for different tungsten thicknesses is shown in Fig. 36. For all target thicknesses the momentum peaks around $5-10 \mathrm{MeV} / \mathrm{c}$, which the number of emitted positrons decrease with the decreasing target thickness in this 
plot. Only from $1 \mathrm{~mm}$ to $6 \mathrm{~mm}$ tungsten thickness plots are shown.

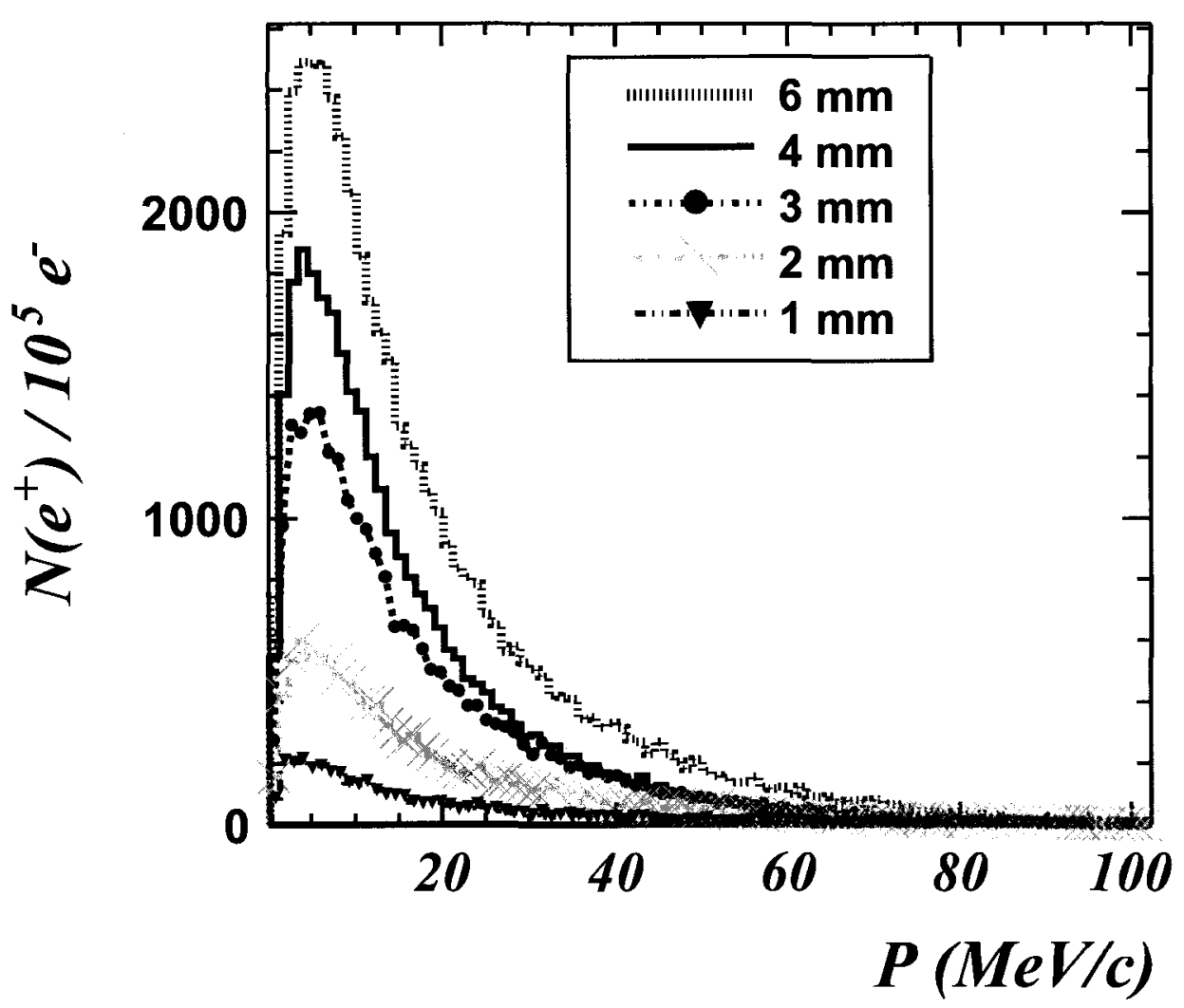

FIG. 36: The momentum distribution of the positrons for different tungsten thicknesses. The highest number of positrons emerge from $6 \mathrm{~mm}$ target, number of positrons decrease linearly with the decreasing target thickness.

\section{Selection of target thickness and deposited power}

In Fig. 37, on the left axis, the total efficiency of positron conversion as a function of tungsten thickness is shown with filled circle symbols. At this given incoming electron beam momentum $p\left(e^{-}\right)=126 \mathrm{MeV} / \mathrm{c}$, the number of positrons peaks at $\sim 6 \mathrm{~mm}$ tungsten thickness, which there are no cuts applied to the emerging positrons. At $6 \mathrm{~mm}$ tungsten, statistically 1 positron will emerge (at a random kinetic energy varying from $\sim 0$ to $120 \mathrm{MeV}$ ) from every 3 electrons; and at $2 \mathrm{~mm}$ thickness 1 positron is created from 10 incoming electrons. In the same figure, on the right axis, the brightness as a function of tungsten thickness is plotted with filled squares. The positron conversion efficiency reaches its maximum cascade at $6 \mathrm{~mm}$ as seen in the 
figure but when we look at the efficiency after $100 \mathrm{mrad}$ hard cuts (Brightness), the maximum number of positrons peaks around $2-4 \mathrm{~mm}$ tungsten $\left(\sim 1 X_{0}\right)$.

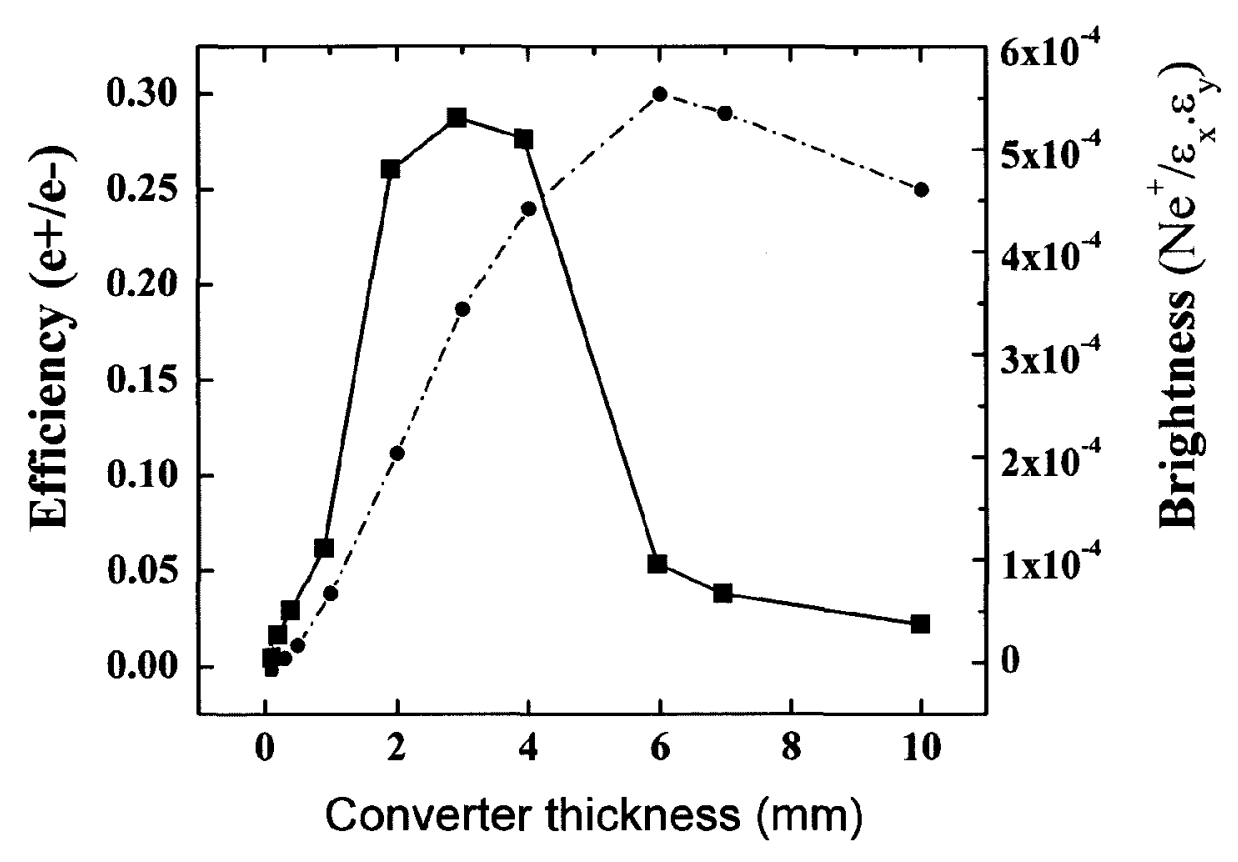

FIG. 37: On the left axis, the conversion efficiency (ratio of all created positrons per incoming number of electrons) as a function of tungsten thickness is shown with filled circles. The electron beam momentum is $126 \mathrm{MeV} / \mathrm{c}$. On the right axis, the brightness is plotted with filled squares. The applied hard cut numbers to create this figure are $\theta<100 \mathrm{mrad}$ per $\mathrm{MeV}$. The momentum cut was $15 \pm 0.5 \mathrm{MeV} / \mathrm{c}$ (r.m.s). The lines are drawn to guide the eye.

In Fig. 38, the percentage of deposited power in the tungsten as a function of the tungsten thickness is shown. The vertical axis is in percentage of the incoming beam power. For instance, with $126 \mathrm{MeV}$ beam at $10 \mathrm{~mA}$ current the incoming electron beam will carry 1.2 MW beam power. If a $3 \mathrm{~mm}$ tungsten is used, $10 \%$ of this 1.2 MW power, which is $120 \mathrm{~kW}$ will be deposited in the tungsten. At $2 \mathrm{~mm}$, it is shown that about $5 \%$ of the initial electron beam power, $\sim 60 \mathrm{~kW}$, will be deposited in the tungsten. The rest of the power mostly flows downstream of the tungsten target, which is covered in more details in Chapter V.

Figures 37 and 38 are two important optimization plots, which led us to decide what thickness may be used for the target thickness. After analyzing these plots, 


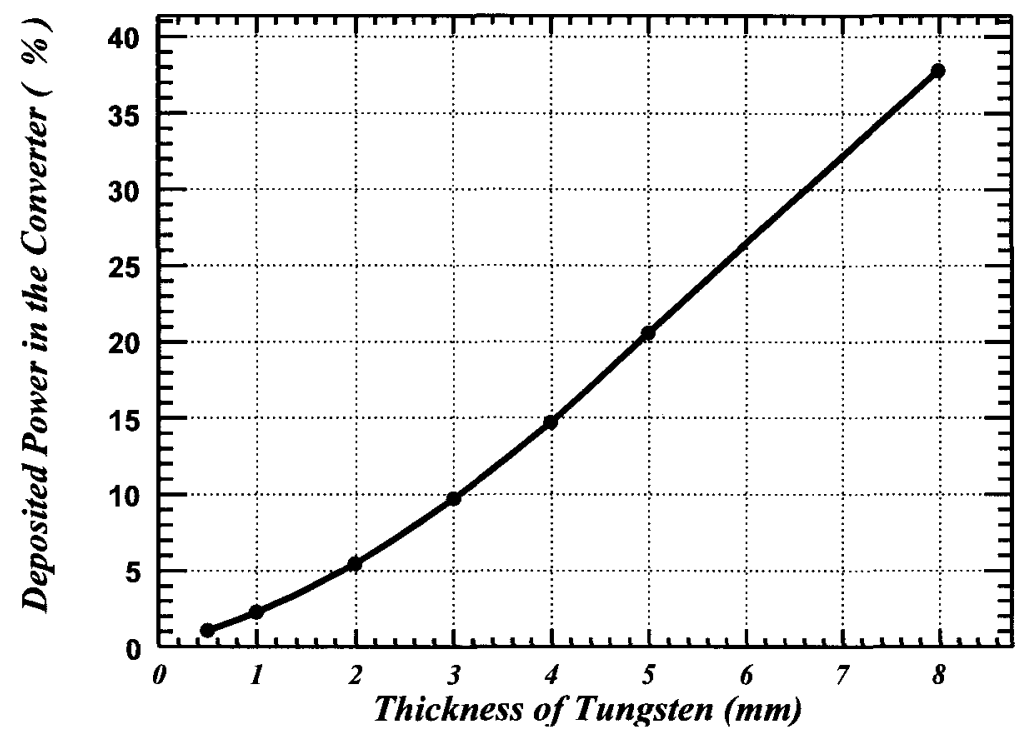

FIG. 38: The percentage of the deposited power w.r.t the incoming beam power as a function of the tungsten thickness is plotted. The power deposition grows linearly with the increased thickness. The rest of the power (power from the secondary particles such as electrons, positrons, gamma rays and neutrons) flows downstream and upstream of the tungsten target. For example at $2 \mathrm{~mm}$ tungsten, $\sim 5 \%$ of the incoming beam power is deposited in the tungsten itself.

we have concluded that a target thickness of $2 \mathrm{~mm}$ tungsten has much less power deposited when compared to $3 \mathrm{~mm}$ tungsten with comparable positron brightness.

\section{Time, momentum and brightness of the positrons}

In Fig. 39(a), the momentum distribution of the positrons from a $2 \mathrm{~mm}$ tungsten is shown. The same positrons filtered via a brightness filter of $\left|\theta_{x}, \theta_{y}\right|<100 \mathrm{mrad}$, which are plotted in Fig. 39(b). The maximum number of positrons without any cuts is around $5-10 \mathrm{MeV} / \mathrm{c}$, but the maximum number of positrons appear between 15-25 MeV/c when the $100 \mathrm{mrad}$ transverse cuts are applied as seen in Fig. 39(b).

In Fig. 40, the time distribution of the positrons right after the target is plotted. The distribution has a very long tail extending up to $400 \mathrm{ps}$ away from the peak value (without any cuts). The FWHM is approximately 0.4 ps. 


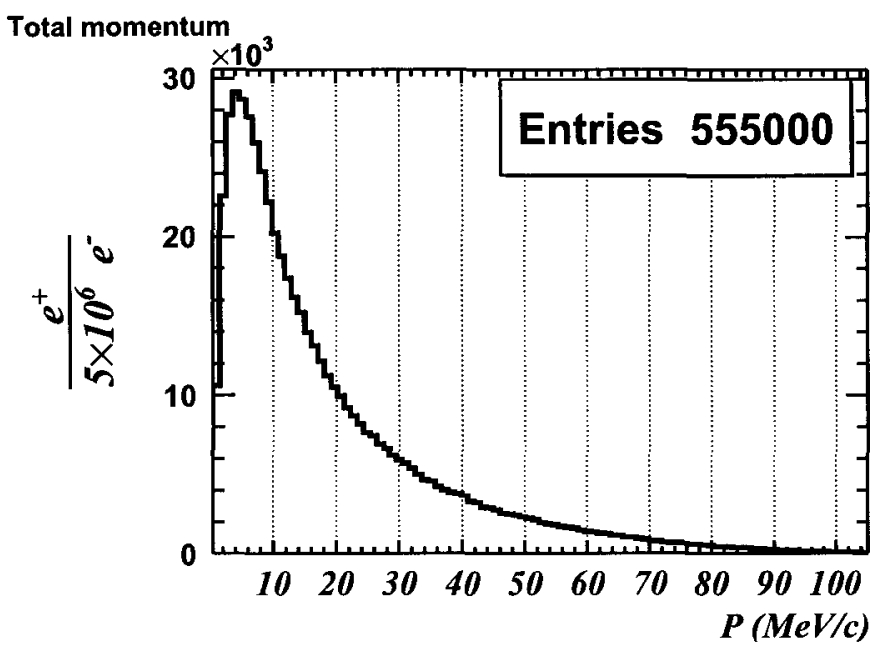

(a)

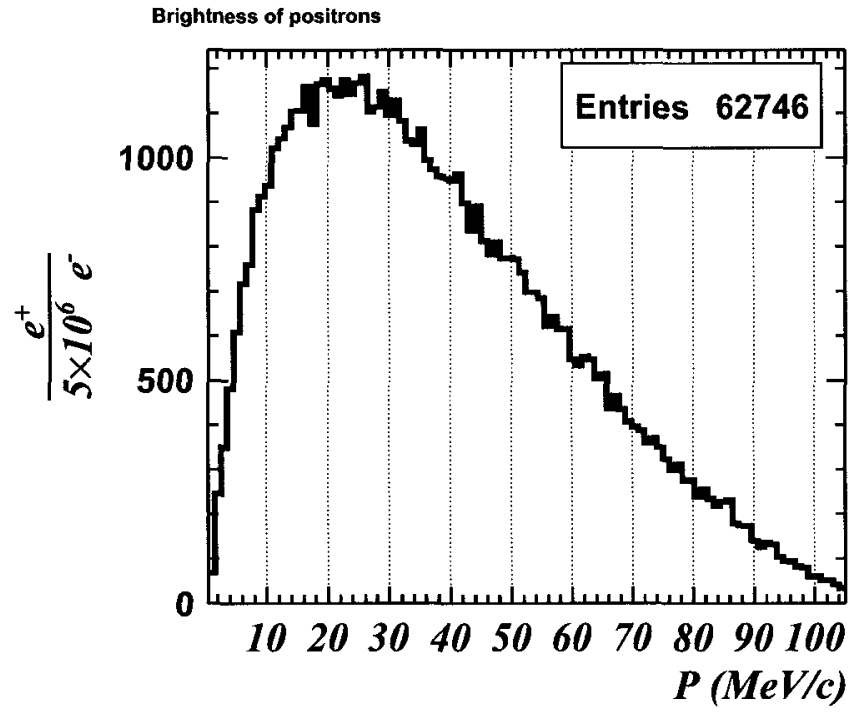

(b)

FIG. 39: (a) The total momentum of the positrons emerging from a $2 \mathrm{~mm}$ tungsten converter. The momentum distribution of positrons shows a non-gaussian beam profile. The incoming electron beam is $126 \mathrm{MeV} / \mathrm{c}$. The positron momentum peaks around $5-10 \mathrm{MeV} / \mathrm{c}$. To simulate this plot, $5 \times 10^{6}$ electrons are used and as a result $5.55 \times 10^{5}$ positrons are obtained via the pair production. (b) The same plot with the brightness filter is applied. The applied hard cut number to create this figure is $\theta<100 \mathrm{mrad}$ in $\mathrm{x}$ and $\mathrm{y}$. This brightness plot has broad peak around $15-25 \mathrm{MeV} / \mathrm{c}$. 


\section{Time distribution of positrons}

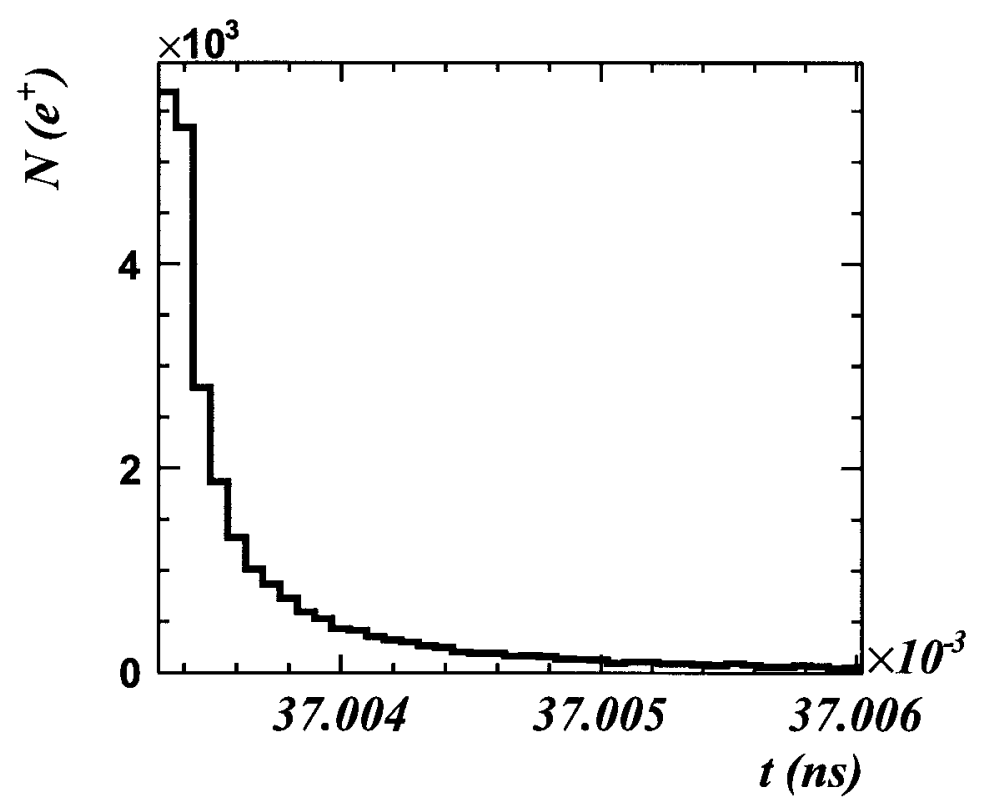

FIG. 40: The time (t) distribution of the positrons right after a $2 \mathrm{~mm}$ tungsten target is plotted. The distribution is non-gaussian with FWHM $\sim 0.4 \mathrm{ps.} \mathrm{The} \mathrm{tail} \mathrm{is} \mathrm{very}$ long, where the plot is zoomed in to see the peak more clearly. 


\section{Transverse and longitudinal phase space profiles of the positrons}

From Fig. 41 to 44, the transverse and longitudinal phase space plots of the positrons right out of the tungsten target are plotted. These plots have no cuts on them, showing the raw simulated data. The $p-\theta$ distribution is shown in Fig. 41(a), where positrons accumulated the highest number in the area at $p<30 \mathrm{MeV} / \mathrm{c} \otimes \theta<$ $\pm 100 \mathrm{mrad}$ ). In Fig. 41(b), the longitudinal phase space $(p-t)$ distribution is shown, where it is seen that $p$ and $t$ are not correlated at the target. In Fig. 42, $x$ and $y$ position distributions of the positrons are plotted. The distributions are gaussian types with moderate tails. The rms values in transverse positions are $x_{r m s} \sim 0.24$ mm. A gaussian fit up to $2-\sigma$ gives improved rms values with $\sigma_{x}$ (fit) $0.15 \mathrm{~mm}$. The transverse angles $x^{\prime}$ and $y^{\prime}$ are plotted in Fig. 43. As similar as in $x$ and $y$, angles are gaussian type distributions with $\theta_{r m s} \sim 400 \mathrm{mrad}$. Because of the long tails, the distributions statistics are biased with this large rms values. Fitting a gaussian up to 2- $\sigma$ shows about a factor of three improvement with new fit values $\sigma_{\theta}($ fit $) \sim 150 \mathrm{mrad}$. As seen in the figures, the $p$ and $t$ distributions are non-gaussian, while transverse position and angle distributions are gaussian type distributions. In further design studies, we are going to apply a hard cut to these outputs and select a portion of these positrons according to these gaussian fits. In Fig. 44, $x-x^{\prime}$ and $y-y^{\prime}$ phase space areas of positrons emitted from the tungsten are shown. In the plots, a very small correlation is seen. This is due to the fact that the positrons are created in the tungsten target travel in the tungsten to the surface, which is basically a drift space. This travel in the drift space (in the $2 \mathrm{~mm}$ thick tungsten) gives a slight correlation to the transverse phase space areas. 


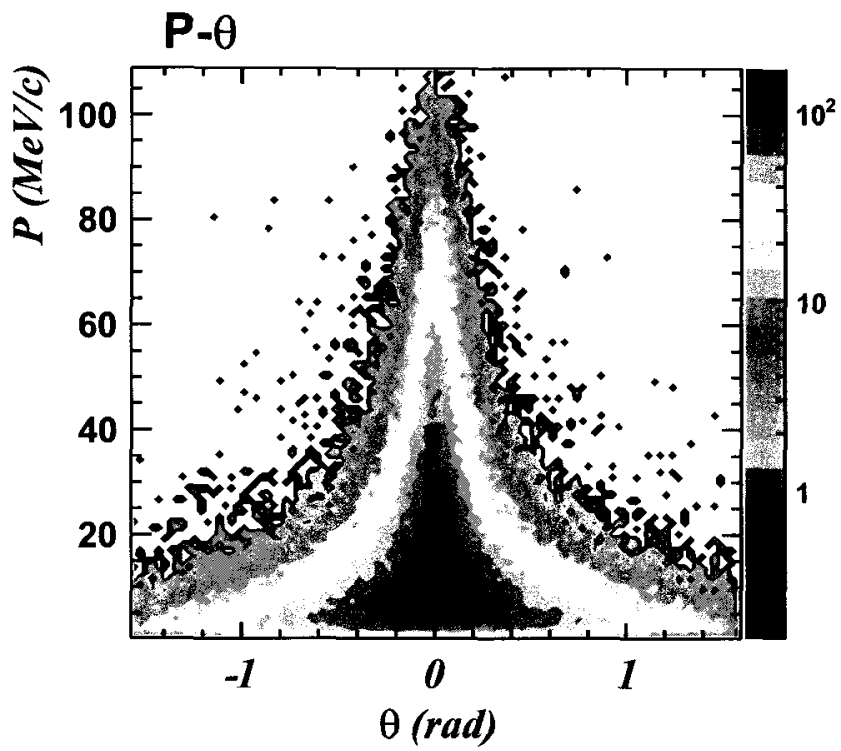

(a)

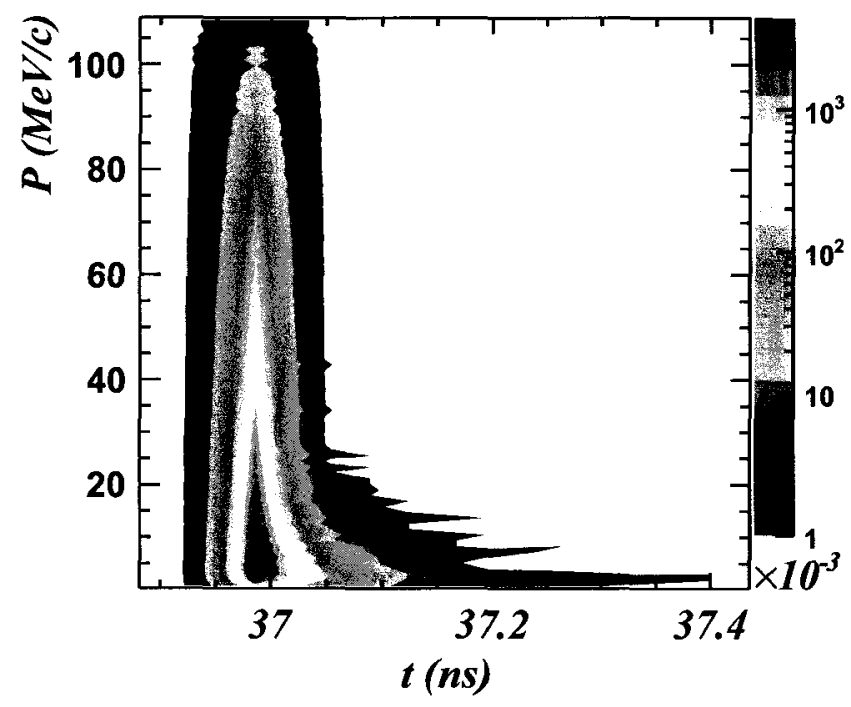

(b)

FIG. 41: $p-\theta$ and $p-t$ distributions of the positrons emerging from a $2 \mathrm{~mm}$ tungsten target without any cuts. (a) $p-\theta$ distribution shows that the highest number of positrons emerge at 0 radian with a broad momentum range between $5-30 \mathrm{MeV} / \mathrm{c}$. (b) $p-t$ distribution shows uncorrelated longitudinal profile. Here the tail extends away from the peak up to $400 \mathrm{ps}$. 


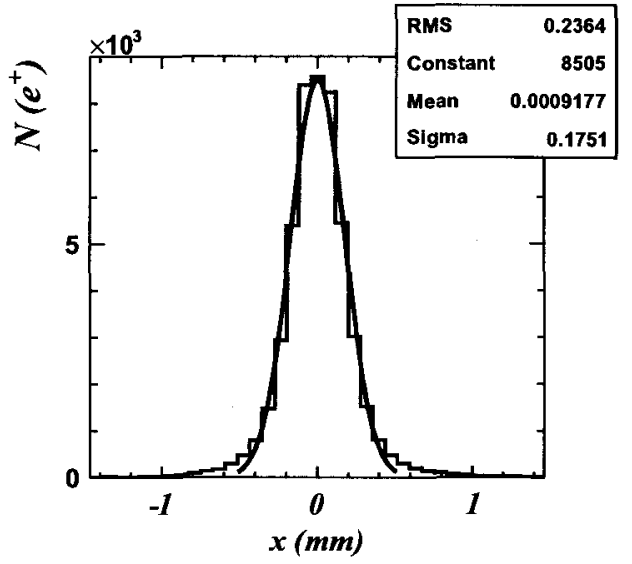

(a)

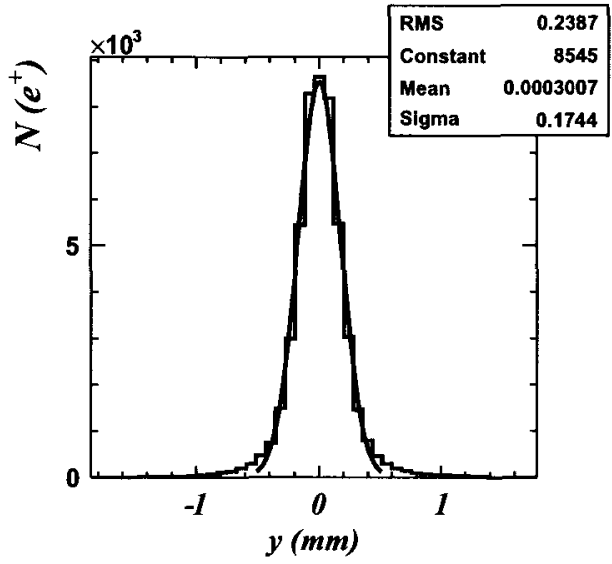

(b)

FIG. 42: $x-y$ transverse position distributions of the positrons emerging from a $2 \mathrm{~mm}$ tungsten target without any cuts. Incoming electron beam size is $\sigma=0.1 \mathrm{~mm}$. (a) $x$ profile is close to a gaussian profile. A gaussian fit to 2- $\sigma$ is plotted only to the peak portion giving a fit $\sigma \sim 0.17 \mathrm{~mm}$. (b) Same method of fitting is used in $y$ profile, which the gaussian fit is giving a $\sigma \sim 0.17 \mathrm{~mm}$.

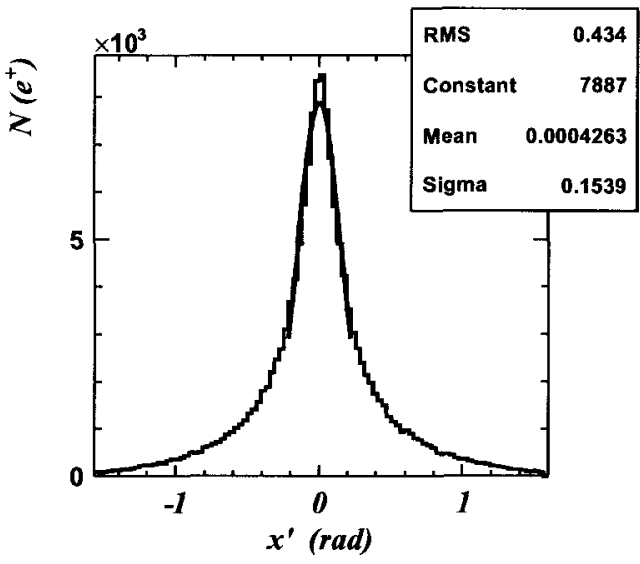

(a)

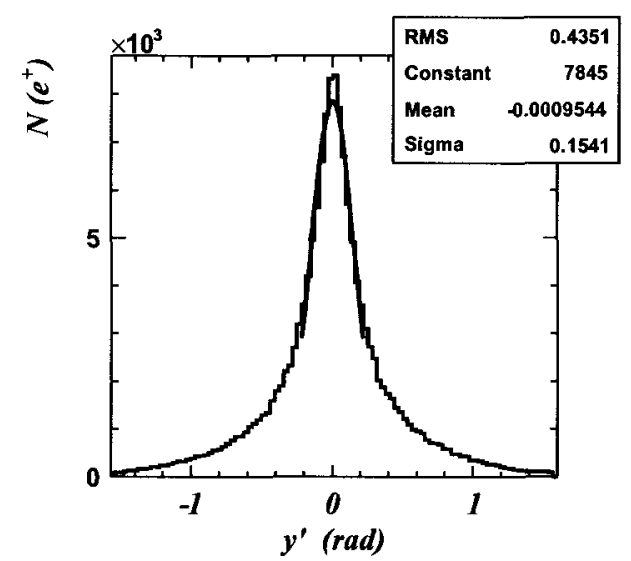

(b)

FIG. 43: $x^{\prime}-y^{\prime}$ distributions of the positrons emerging from a $2 \mathrm{~mm}$ tungsten target without any cuts. (a) $x^{\prime}$ profile shows close to a gaussian profile. A gaussian fit is plotted only 2- $\sigma$ to the peak portion resulting a fit $\sigma \sim 150 \mathrm{mrad}$ (b) $y^{\prime}$ profile shows close to a gaussian profile. Same method used for gaussian fit resulting a fit $\sigma \sim 150$ mrad. 


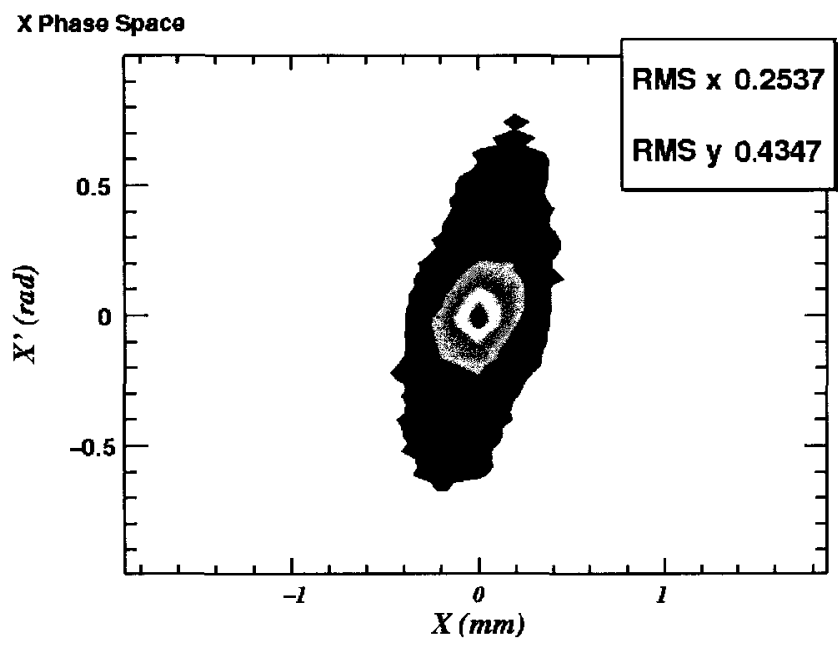

(a)

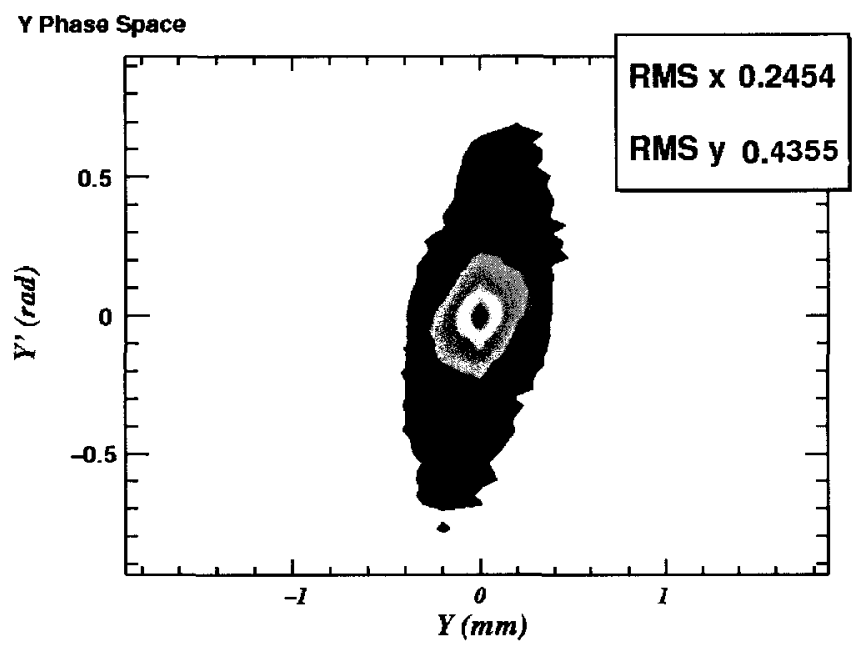

(b)

FIG. 44: $x-y$ transverse phase space distributions of the positrons emerging from a $2 \mathrm{~mm}$ tungsten target without any cuts. (a) $x-x^{\prime}$ profile is slightly tilted with a very small correlation. (b) $y-y^{\prime}$ also shows a small correlation between position and angle. This correlation is due to the multiple scattering from the tungsten target. The thicker the target the bigger the correlation will be. 


\section{The incoming electron beam spot size}

The electron spot size used in the simulations is given as $100 \mu \mathrm{m}$ in Table 10. It may seem highly unrealistic to bombard the tungsten target with a transverse electron beam size of this small spot size coupled with 1.2 MW beam, the fact is that the positron emittance shows a linear dependence with the increasing electron beam size as shown in Fig. 45. In this figure the transverse emittance of the positrons (after $100 \mathrm{mrad}$ cut) as a function of the incoming electron beam sigma spot size is plotted. The Power $/ \mathrm{mm}^{2}$ on the target is decreased at each beam size increment, but this will cost the number of captured positrons. With an electron spot size on the target $\sigma=0.2 \mathrm{~mm}$, the Power $/ \sigma^{2}$ value is about four times lower than the spot size of $\sigma=0.1 \mathrm{~mm}$. But as it can be seen, the positron emittance is doubled after changing the spot size from 0.1 to $0.2 \mathrm{~mm}$ electron $\sigma$ spot size. We get factor of two less positron current at the target by trading off a factor of four less Power per Area. More detailed discussion about the power deposition and target design issues are introduced in Chapter V.

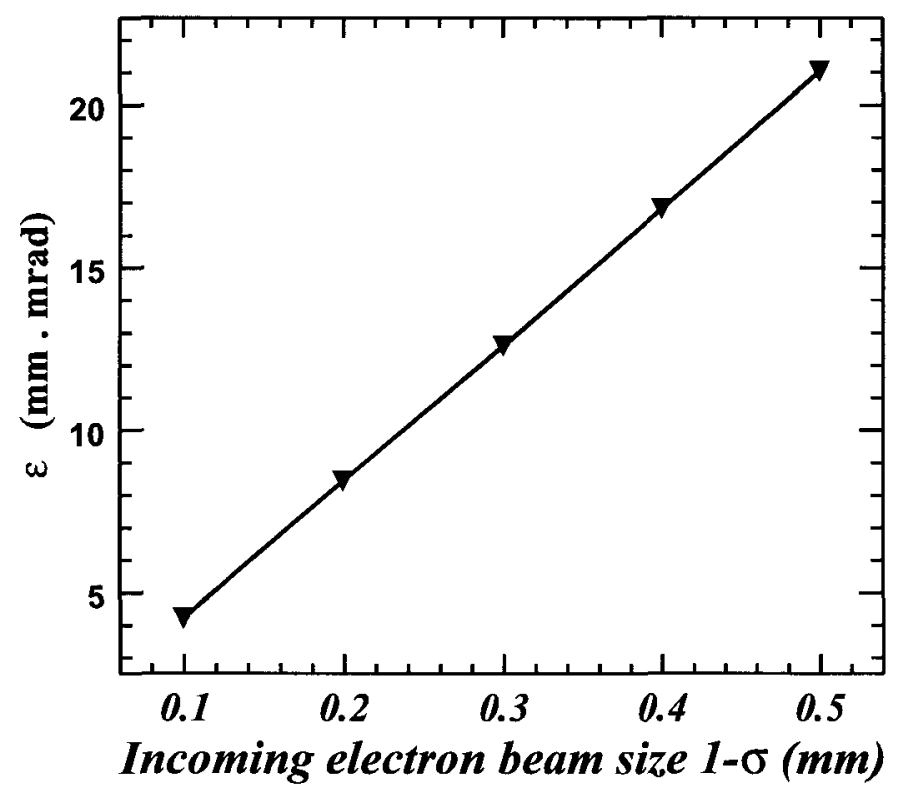

FIG. 45: The emittance of the positrons from a $2 \mathrm{~mm}$ tungsten converter w.r.t the beam size of the incoming electron beam. The emittance of the positrons grow linearly with the electron beam size. The incoming electron beam momentum is 126 $\mathrm{MeV} / \mathrm{c}$, where for positrons a cut in angle is used $(\theta<100 \mathrm{mrad})$. 


\section{Twiss parameters of the selected positrons}

The admittance measurements give a rough estimation for the allowed phase space area of the positrons at the North Linac connection point (let me call this point $\left.s_{2}\right)$. For design considerations, Admittance $(A)$ for transverse components $A_{x}\left(s_{2}\right)=$ $A_{y}\left(s_{2}\right)=10 \mathrm{~mm}$.mrad are used. At the same connection point, the positron injection momentum is required to be $\sim 126 \mathrm{MeV} / \mathrm{c}$. By using the normalized emittance, which is an invariant of the motion under acceleration, the total phase space area of the selected positrons at the target can be calculated. Here the North Linac connection point $\left(s_{2}\right)$ and target location $\left(s_{1}\right)$ have the following relation:

$$
\begin{gathered}
\pi \varepsilon_{N}=\pi \gamma_{1} \beta_{1} \varepsilon_{1}=\pi \gamma_{2} \beta_{2} \varepsilon_{2} \\
A_{N}=\gamma_{1} \beta_{1} A_{1}=\gamma_{2} \beta_{2} A_{2}
\end{gathered}
$$

where $\varepsilon_{N}$ is the normalized emittance and $\gamma, \beta$ are relativistic factors. The area of the total geometrical phase space is $A=\pi \varepsilon$. The $\varepsilon$ is set of emittance areas, which falls into the total phase space area of the beam. This number is usually introduced as 1- $\sigma$ emittance $\left(\varepsilon_{r m s}\right)$. For example at CEBAF, the total phase space area is given by 4 sigma of the r.m.s emittance $A=4 \varepsilon_{r m s}$. At the target, from the large phase space area of the all positrons, we can select a useful portion of these emitted positrons and design beamline lattices according to this selected positron beam twiss parameters. Such a selection process can be done by using Eq. (36), where any positron which satisfies the provided equation with the desired twiss parameters falls into the admittance phase ellipse. This selection process is illustrated in Fig. 46(a).

As it is seen from the Table 11, there is not much difference in terms of $\mathrm{e}^{+}$number for $15 \mathrm{MeV}$ bin or $40 \mathrm{MeV}$ bin. $15 \mathrm{MeV}$ selection is more favorable since by the time the positrons reach to the North Linac, their emittance will be reduced by a factor

of $\left(\frac{15}{126}\right)$, where for $40 \mathrm{MeV}$ positrons the emittance is reduced much lower than that by a factor of $\left(\frac{40}{126}\right)$.

If we select $15 \mathrm{MeV} \mathrm{e}^{+}$at the target, then their corresponding total phase space area is $\sim 84 \mathrm{~mm}$.mrad. The selected positrons have a gaussian type distribution in position and non-gaussian distribution in angle (almost flat). The phase space area of the selected positrons is shown in Fig. 46(b). For optics calculations, we use 1- $\sigma$ emittance of the selected positrons at the target, which can also be related to the 
total phase space area by:

$$
\begin{aligned}
A & =\pi a b \\
& =\pi\left(3 \sigma_{x}\right)\left(\sqrt{3} \sigma_{x^{\prime}}\right) \sqrt{1-\alpha^{2}} \\
& =\pi 3 \sqrt{3} \varepsilon_{r m s}
\end{aligned}
$$

To get the maximum current, the captured angle must be as much as possible which the outgoing spot size must be as much as smaller. With a positron $\sigma_{x y} \sim 0.12 \mathrm{~mm}$ and $\sigma_{x^{\prime} y^{\prime}} \sim 44 \mathrm{mrad}$ with the correlation $\alpha \sim 0$, this results in an r.m.s emittance $\varepsilon \sim 5 \mathrm{~mm}$.mrad at the target. In Table 11, the number of positrons within $\varepsilon \sim 5$

TABLE 11: Selection of positrons within the trial emittance value $\varepsilon \sim 5.0 \mathrm{~mm}$.mrad. The momentum of the positrons is $P \pm 0.5 \mathrm{MeV} / \mathrm{c}$ (r.m.s), and $10^{7} \mathrm{e}^{-}$is used to create these $\mathrm{e}^{+}$.

\begin{tabular}{ccccccc}
\hline \hline $\begin{array}{c}\mathrm{W} \\
(\mathrm{mm})\end{array}$ & $\begin{array}{c}\beta(x, y) \\
(\mathrm{m})\end{array}$ & $\alpha_{x}$ & $\alpha_{y}$ & $\begin{array}{c}\varepsilon(x, y) \\
(\mathrm{mm} . \mathrm{mrad})\end{array}$ & $\begin{array}{c}P \\
(\mathrm{MeV} / \mathrm{c})\end{array}$ & $\begin{array}{c}e^{+} \\
\mathrm{count}\end{array}$ \\
\hline 2 & 0.0033 & -0.006 & -0.0221 & 5.1 & 5 & 1210 \\
2 & 0.0033 & -0.11 & -0.214 & 5.3 & 15 & 3307 \\
2 & 0.0031 & -0.0436 & -0.075 & 5.3 & 40 & 2850 \\
2 & 0.0033 & -0.135 & -0.236 & 5.3 & 60 & 1071 \\
\hline 3 & 0.0034 & -0.012 & -0.034 & 5.1 & 5 & 1297 \\
3 & 0.0036 & -0.08 & -0.15 & 5.1 & 15 & 3350 \\
3 & 0.0036 & -0.095 & -0.17 & 5.2 & 40 & 3201 \\
3 & 0.0036 & -0.147 & -0.346 & 5.1 & 60 & 1105 \\
\hline 4 & 0.0036 & -0.044 & -0.133 & 5.2 & 15 & 3105 \\
4 & 0.0037 & -0.123 & -0.336 & 5.1 & 60 & 905 \\
\hline \hline
\end{tabular}

mm.mrad for different tungsten thicknesses for various positron momentum bins are shown. As clearly seen before from the brightness selection process, the number of positrons within this tight emittance area varies with momentum of the selected positrons. The twiss parameters of the selected positrons are slightly different than the pre-defined twiss parameters $\left(\beta_{0}=0.003 \mathrm{~m}, \alpha_{0}=-0.1, \varepsilon_{0}=5 \mathrm{~mm} . \mathrm{mrad}\right)$ due to the statistical fluctuations in the selection process.

In the following sections, design simulations are carried out by using the optimized numbers and admittance results. For tungsten converter a thickness of $2 \mathrm{~mm}$ is used. The incoming electron beam parameters in Table 10 are used. 


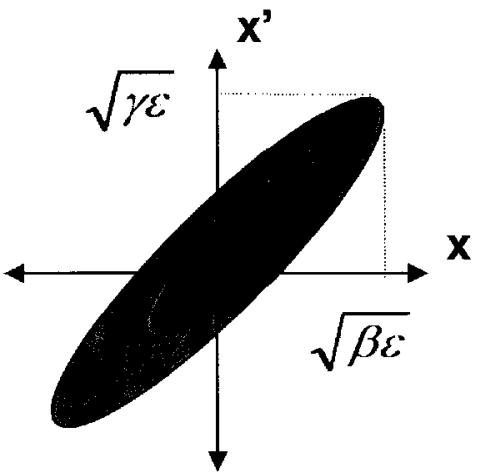

(a)

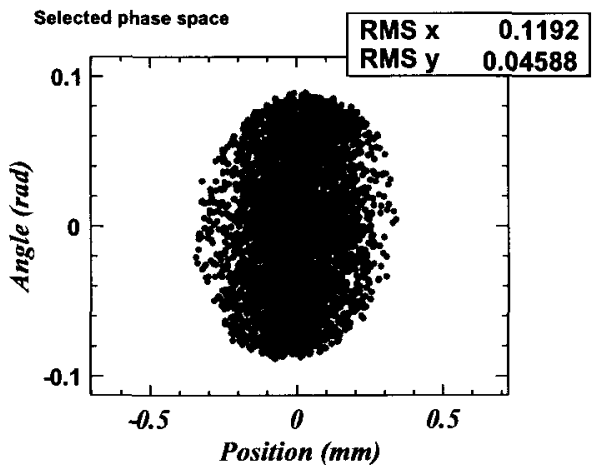

(b)

FIG. 46: The whole ellipse represents the $100 \%$ of the beam which is the admittance area. To design a lattice most simulation tools require the user to input 1- $\sigma$ emittance. In ELEGANT and OPTIM simulations, we use $\varepsilon=A d m i t t a n c e / 3$ as our input as illustrated in (a). In G4BEAMLINE simulations we use the cut positrons as the input, which is $100 \%$ of the selected beam as shown in (b).

The optimal target thickness yielding the maximum number of positrons within calculated acceptance area at the target and the optimization for the momentum bin are completed. Then how many positrons are there within these optimized parameters that can be used to transport from a converter target to the North Linac connection point? By using the selection process, the efficiency is given:

$$
\frac{36000 e^{+}\left(15<P\left(e^{+}\right)<25 \mathrm{MeV} / \mathrm{c}\right)}{10^{7} e^{-} .10 \mathrm{MeV} / \mathrm{c}}=3.6 \times 10^{-4}\left[e^{+} \text {per } e^{-} \text {per } \mathrm{MeV}(\mathrm{rms})\right]
$$

For a positron beam with an r.m.s emittance of $\varepsilon \sim 5 \mathrm{~mm} \cdot \mathrm{mrad}$ with a momentum $15 \pm 0.5 \mathrm{MeV} / \mathrm{c}(\mathrm{rms})$, there are about $3.6 \times 10^{3} \mathrm{e}^{+}$per $10^{7} \mathrm{e}^{-}$per $\mathrm{MeV}$, which this is equal to an efficiency of $3.6 \times 10^{-4}$. This efficiency corresponds to a $\mathrm{e}^{+}$current of $3.6 \mu \mathrm{A}$ for $10 \mathrm{~mA}$ incoming $\mathrm{e}^{-}$beam. For the lattice design inputs, we use the whole selected positron beam given in Fig. 46(b) as an input in the G4BEAMLINE, while in other simulation codes $\varepsilon=$ Admittance $/ 3$ is used as the input value. In the design studies, we will try to transport all of these selected positrons.

\section{IV.2 QUARTER WAVE TRANSFORMER (QWT)}

A solution for the CEBAF positron source must capture as much as positrons in the acceptable $6 \mathrm{D}$ phase space. There are two widely used types of solenoids to 
collect positrons at the first stage; Quarter Wave Transformer (QWT) and Adiabatic Matching Device (AMD). The AMD, which is a tapered solenoid, accepts more energy band; on the other hand the QWT accepts a larger amount of transverse momentum. As measured in the ARC1, the fractional energy ratio of $3 \times 10^{-3}$ is the maximum tolerated spread. With the $12 \mathrm{GeV}$ upgrade the upgraded energy will have reached to $1 \mathrm{GeV}$ before ARC1. This type of restriction limits us on how much energy spread we can get at the positron conversion target, but in fact we can get as much as transverse $p_{\perp}$ and transform it to a transverse position spread, which is possible with a QWT. To get enough current one needs to consider either increasing the driving beam current (and/or energy) or capture more transverse momentum.

The QWT is actually structured from a short lens with a high magnetic field , usually 5-20 Tesla peak in pulsed sources, and followed by a long solenoidal section extending over a few meters including the accelerator section. Usually the long solenoid surrounds the accelerator cavities. The field profile of the QWT is illustrated in Fig. 47.

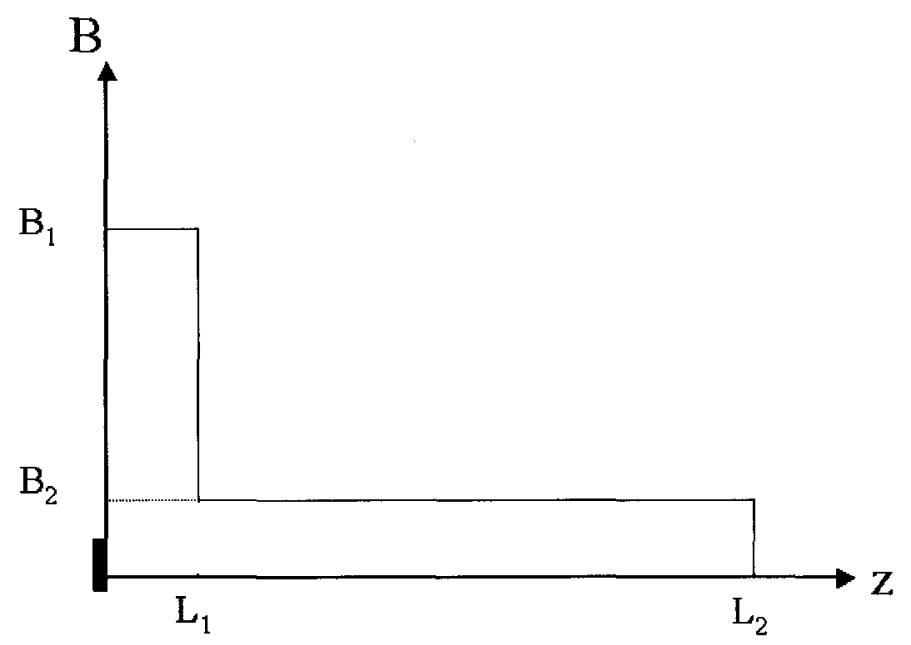

FIG. 47: The short and long solenoidal field profile of the QWT. The target converter is assumed to be positioned at the origin. Here $B_{1}$ and $B_{2}$ are the field strengths and $\mathrm{L}_{1}$ and $\mathrm{L}_{2}$ are the lengths of the short and long solenoids respectively.

In the $\left(\mathrm{x}, p_{x} ; \mathrm{y}, p_{y}\right)$ plane the acceptance volume can be expressed as a function of Larmor frequency with a constant uniform magnetic field:

$$
V\left(\chi_{1}\right)=\frac{2 \pi^{2}}{3}\left(\frac{e B_{2} a^{2}}{2}\right) \times\left[1-\left(1-\frac{1}{\sin ^{2} \chi_{1}+\left(B_{1} / B_{2}\right)^{2} \cos ^{2} \chi_{1}}\right)^{3 / 2}\right]
$$


where $\chi_{1}=\frac{300 B_{1}(\mathrm{Tesla}) L_{1}(\mathrm{~m})}{2 P(\mathrm{MeV} / \mathrm{c})}$ is the Larmor angle, $a$ is the half-aperture of the solenoid and $P$ is the scalar momentum of the positrons. The acceptance volume $(V)$ for a QWT system is calculated for $\chi_{1}=\pi / 2$;

$$
V=\frac{2 \pi^{2}}{3}\left(\frac{e B_{2} a^{2}}{2}\right)
$$

The solenoid transforms the angle spread to a spatial spread. As for CEBAF requires $\mathrm{CW}$ operation, the maximum solenoid field can go as high as $1 \mathrm{~T}$ for $\mathrm{CW}$ operation with current technology without superconducting cooling. Certainly, if cryogenic cooling is considered, then this field value can easily go much higher, but the necessity to position the solenoid right after the converter target makes it almost impossible to preserve the cryogenic temperature. The only modification we are making to QWT system will be not using the long solenoid coupled with warm RF, but to use only the short part. Here the fringe field of the solenoid acts as the $B_{2}$ field. As it can be seen from the equation above that the Larmor angle only depends on the short solenoid $\left(\mathrm{B}_{1}\right)$ at $\chi_{1}=\pi / 2$. Immediately after that, other optics systems will be positioned to clean the beam and then send it to SRF. Current technology for a CW warm RF allows only for a up to $1-2 \mathrm{MV} / \mathrm{m}$ which is not enough to reduce the emittance in a short distance. That disadvantage factors out warm RF from the design infrastructure as well. Superconducting RF is known to be not working when solenoidal field is immersed in it. Also SRF will be very close to the target area which will be absorbing all the power coming from the target. As a result SRF can not be positioned close to the target area in lieu of warm RF.

TABLE 12: The QWT solenoid trial parameters. 1 Tesla is used since for DC operation without use of cryogenics it is the maximum we can get.

\begin{tabular}{lll}
\hline \hline $\mathrm{B}_{1}$ & 1 & $\mathrm{~T}$ \\
$\mathrm{~B}_{2}$ & 0.2 & $\mathrm{~T}$ \\
$\mathrm{~L}_{1}$ & 16 & $\mathrm{~cm}$ \\
$\mathrm{a}$ & 2 & $\mathrm{~cm}$ \\
\hline \hline
\end{tabular}

If trial values for a QWT solenoid as given in Table 12 are used, then the normalized acceptance volume w.r.t the scalar momentum of the positrons has the following 
form as shown in Fig. 48. As clearly seen, for these given values there is a strong peak around $15 \mathrm{MeV} / \mathrm{c}$. This implies that at the target, positrons with $15 \mathrm{MeV} / \mathrm{c}$ will be selected and captured by the short lens.

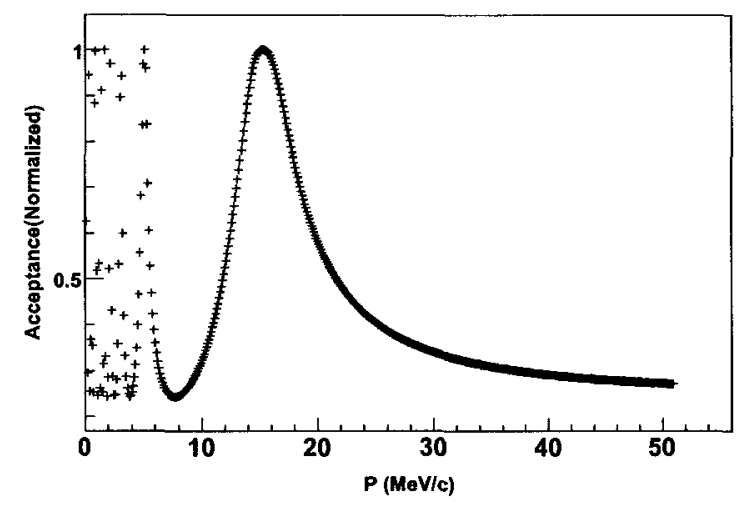

FIG. 48: The acceptance volume of the QWT as a function of central momentum of positrons. The volume is normalized to the $\chi=\pi / 2$ value of the acceptance volume function.

The maximum transverse acceptance for this configuration is calculated as;

$$
\theta_{\max }=x^{\prime}=\frac{e B_{1} a}{2 P}\left(1+\frac{B_{2}}{B_{1}}\right)
$$

From this we get a total $\theta_{\max }=360 \mathrm{mrad}$ capture, but spiralling effect from the solenoid of different energies with large capture angles will increase the time spread substantially, which this will also limit the number of captured positrons. As a safe margin, we can capture $\theta \sim 100 \mathrm{mrad}$ at the target by using QWT.

\section{IV.3 DESIGN - 1 : COMBINED FUNCTION MAGNETS (CFM)}

A Combined Function Magnet (CFM) is a type of magnet where both dipole and quadrupole field components are superpositioned in the same element. The dipole field is a vertically aligned magnetic field and the quadrupole field is vertically(horizontally) defocusing (focusing) for positive signed particles. In the simulation codes, this is achieved by using multipole elements.

In Fig. 49, the G4BEAMLINE snapshot for this proposed CFM is shown. The target is not shown in this figure. The QWT solenoid captures the positrons (electrons). 
The first CFM gives a kick to the selected on-momentum $(15 \mathrm{MeV} / \mathrm{c})$ positrons resulting a deviation from the central orbit an angle $\theta_{1}=9.482$ degrees; the second CFM gives a kick in the opposite direction $\theta_{2}=-8.639$ degrees and the last CFM gives a kick $\theta_{3}=6.893$ degrees. As a result the central orbit and the lattice will deviate from its origin point by $\theta=7.736$ degrees. In this configuration, since electrons get a kick in the opposite direction from the dipole components of the CFM, they will immediately be attenuated in the collimators and magnets at the first collimator.

In Fig. 50, a non-scaled schematic drawing of the proposed solution with combined function magnets is shown. The positions of the elements are close to the values used in the simulations. The total deviation from the origin in transverse direction in this lattice is about $75 \mathrm{~cm}$. In Fig. 51, a CEBAF style quick injector drawing is provided. This schematic gives a sense of this lattice and its optical lattice elements with CFMs, quadrupoles and SRFs. After the CFMs, there are collimators to prevent electrons and other radiation to scatter into the next section where cryomodules are located. The target area must also be completely in a vault as shown in the figure. 


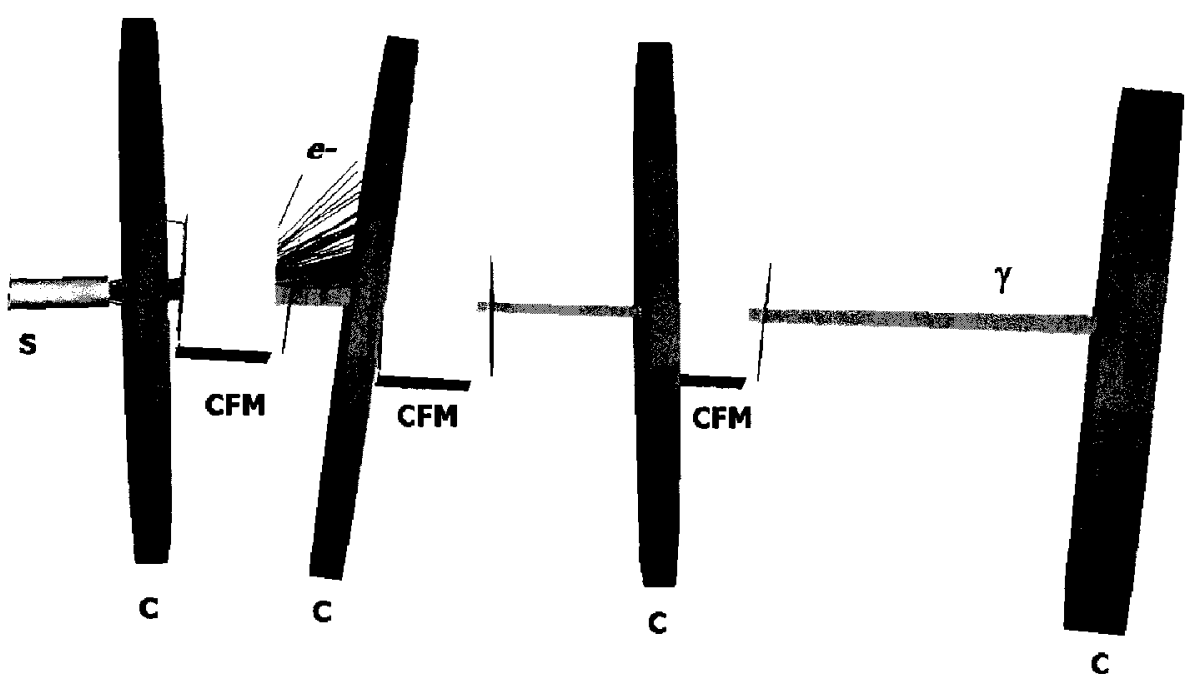

(a)

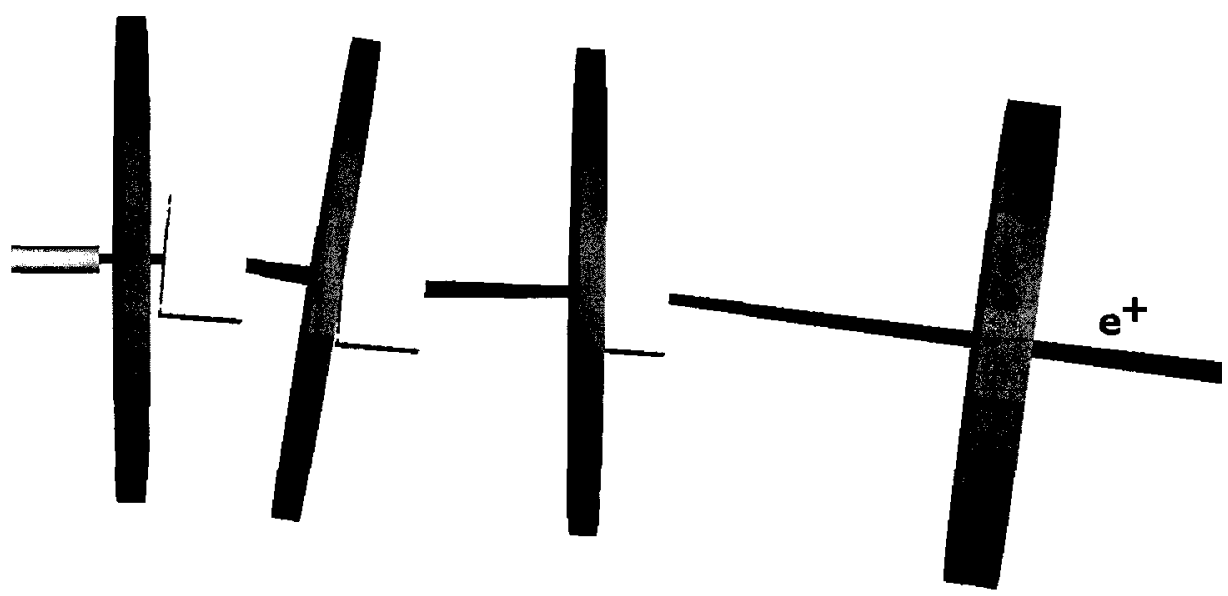

(b)

FIG. 49: A snapshot from the G4BEAMLINE simulation showing the forward secondary particles. The long cylinder on the left is the solenoid (S), the cubes are the combined function magnets (CFM) and the collimators (C) are located in between elements. The solenoid and the combined function magnet captures the positrons. As the bending field diverts opposite signed particles in opposite directions, absorbers and collimators are used to stop electrons. Almost all photons will be stopped as well, but most of the time the simulations fail to show the second scatterings of the photons. (a) $\mathrm{e}^{-}$and $\gamma$ are tracked, where the secondary $\mathrm{e}^{-}$are stopped almost immediately. (b) Only $\mathrm{e}^{+}$beam is shown, where the beam passes cleanly through the holes of collimators. The cryomodule is secured at a far enough location from the radiation zone. In this figure, the particles are intentionally stopped at the collimators to present a more clear picture. When simulating the power deposition in the elements then they are not killed. 


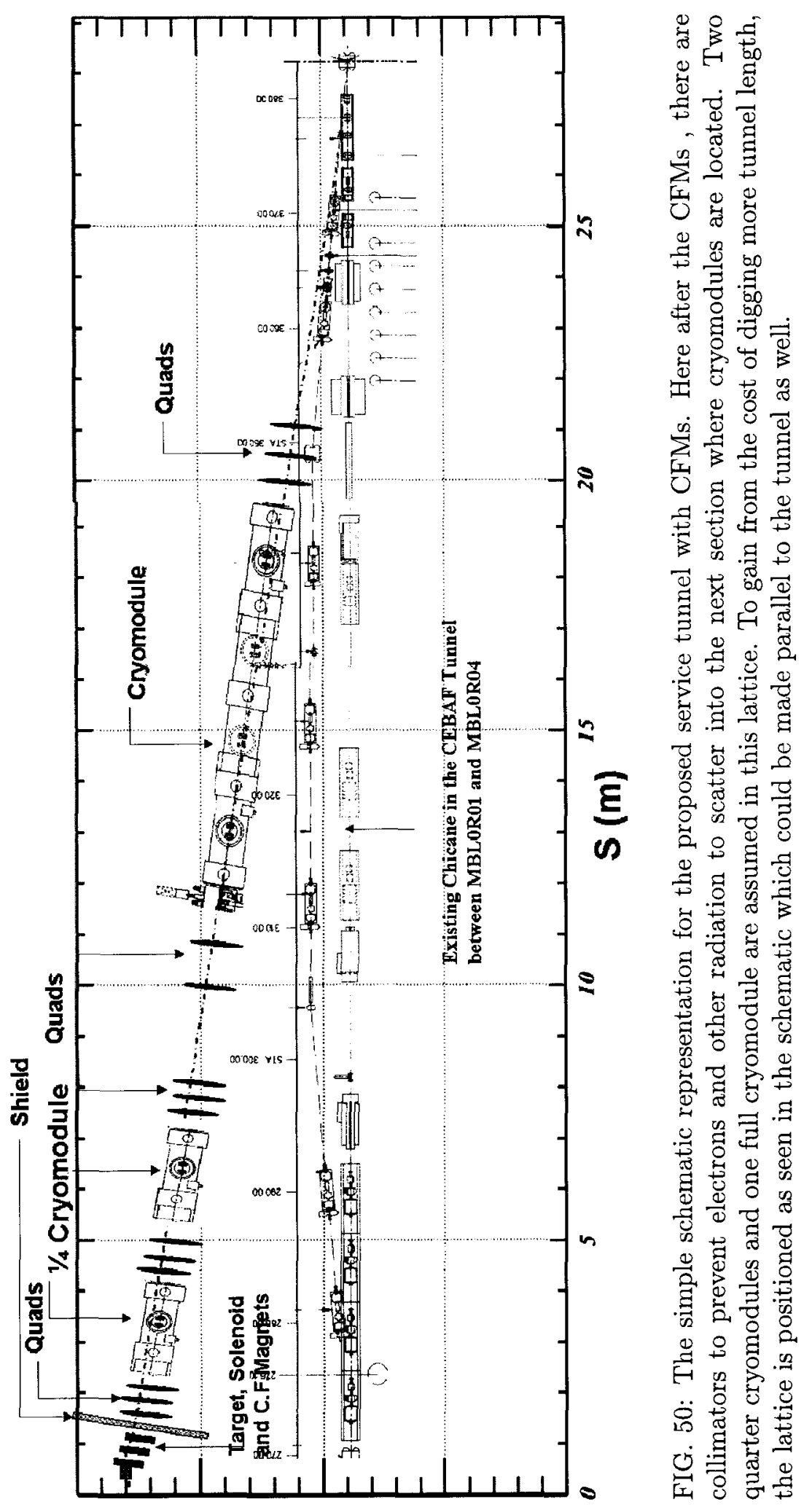




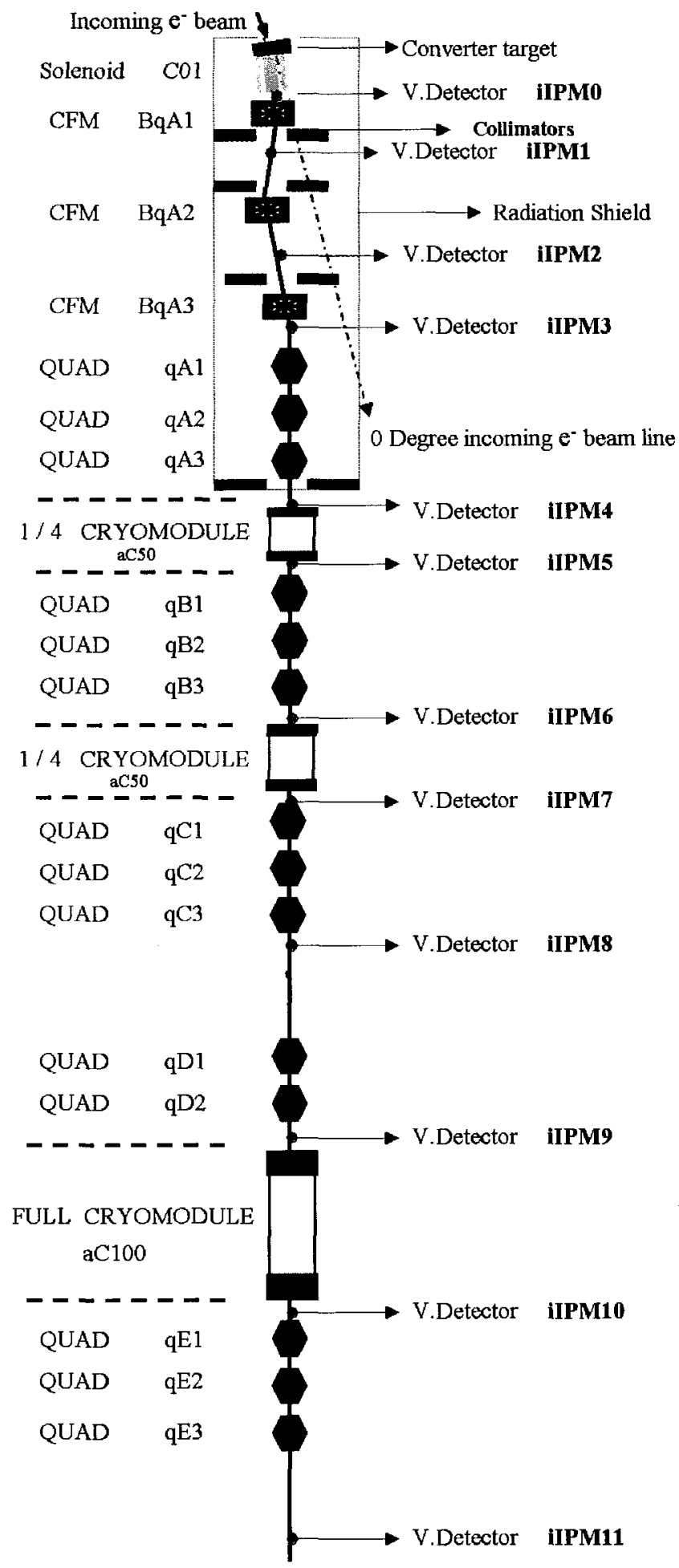

Connection to North Linac

FIG. 51: A quick schematic guide to the CFM lattice. This is similar but much more simplified copy of the quick guide of the CEBAF injector. 


\section{Twiss functions and beam profile of the CFM configuration}

The twiss parameters are convenient tools to track the evolution of the beam in a magnetic lattice. The twiss $\beta$ gives information about the size of the beam, $\gamma$ about the angle and $\alpha$ about the orientation of the beam, whether the beam is converging, diverging or at a waist position. The $\beta$ function is related with the beam size and the emittance as defined in Eq. (38). In addition to the $\beta$, it is also required to look at the positron beam size as the $\sigma$ spot size goes up to a $\mathrm{cm}$ at certain places. In Fig. 52, the $\sigma_{x y}$ size of the positron beam is plotted in the CFM lattice. The $\beta_{x y}$ functions are shown in Fig. 53.

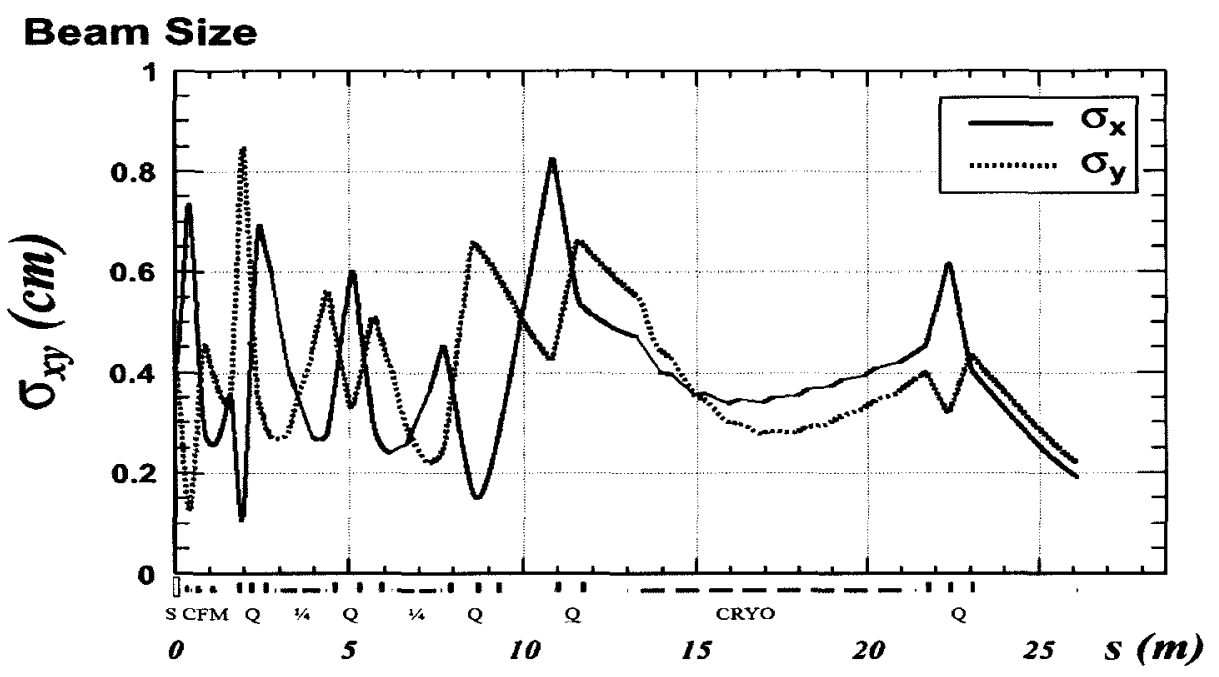

FIG. 52: The 1- $\sigma_{x y}$ beam sizes of the positron beam as a function of the central orbit are plotted in the CFM lattice. The solid line is $\sigma_{x}$ and dashed line is $\sigma_{y}$. The boxes under the horizontal axis show the location of the magnets. The solenoid (S), combined function magnets (CFM), quadrupoles (Q) and quarter cryomodules (1/4) and full cryomodule (CRYO).

The beam size goes up to a $\mathrm{cm}$ at the beginning of the lattice, while it is decreased down to about $2 \mathrm{~mm}$ at the end of the lattice. In Fig. 54, the $\beta_{x y}$ and dispersion $\left(D_{x}\right)$ function are plotted for the first few meters of the CFM lattice. The dispersion is introduced by a dipole field, which has a relation with off-momentum particles as given in Eq. (14). If it is not corrected, the off-momentum particles eventually will be lost in the lattice due to the offset from the design orbit. In Sec. I.1.5, a principle for an achromatic lattice is introduced. Here, in the CFM lattice we follow a similar design strategy. As it is seen from the graph, the $\mathrm{D}_{x}$ goes up to $5 \mathrm{~cm}$ and vanishes 


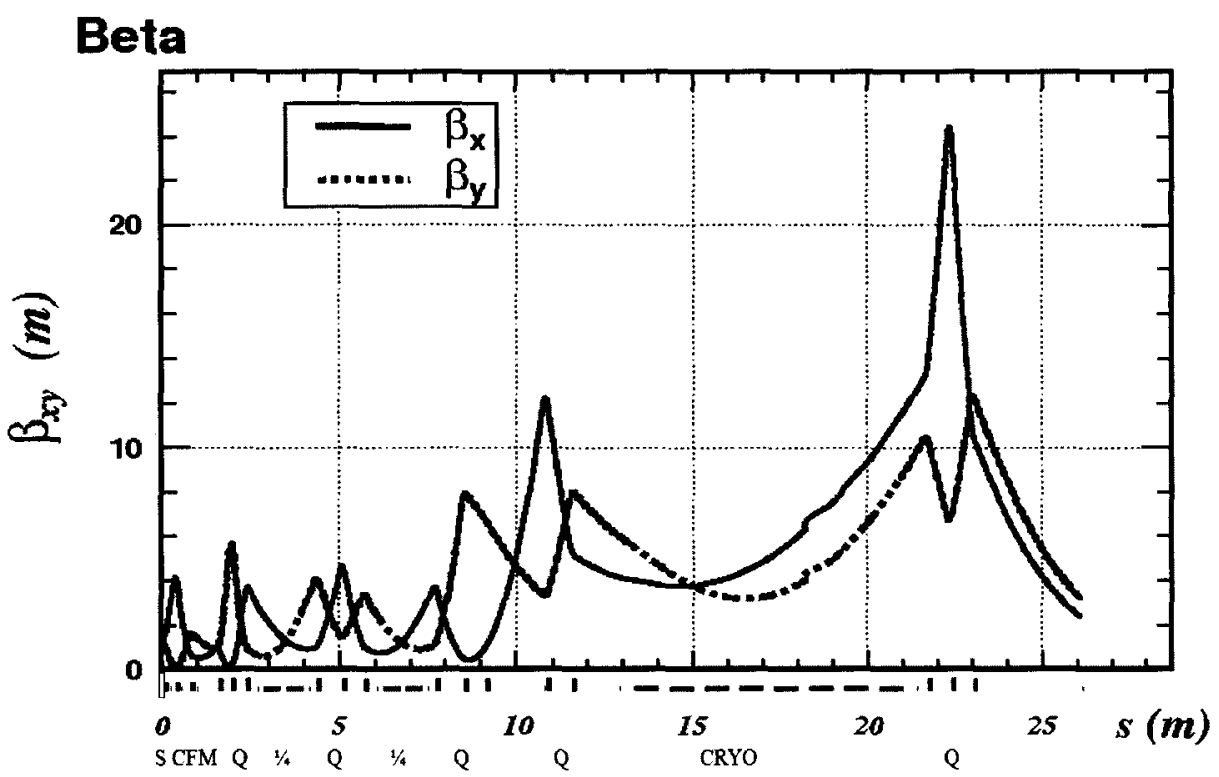

FIG. 53: The $\beta_{x y}$ functions of the positron beam as a function of the central orbit are plotted in the CFM lattice. The solid line is $\beta_{x}$ and dashed line is $\beta_{y}$. The boxes under the horizontal axis show the location of the magnets. The solenoid (S), combined function magnets (CFM), quadrupoles (Q) and quarter cryomodules (1/4) and full cryomodule (CRYO).

at the end of the dipoles. The derivative of the dispersion is zero as well.

The full beam envelopes of the positron beam as plotted in Figs. 55 and 56 , give full information about the beam size. These figures are the result of G4BEAMLINE tracking, which all the particles (selected cut positrons) are plotted. The total beam size goes up to $3 \mathrm{~cm}$ at certain locations. These locations are usually the mid-points of the quadrupoles. As it can be seen from the graphs, the beam sizes are the lowest in size in the cryomodules. Since the full cryomodule is long $(8 \mathrm{~m})$, the beam is brought to a maximum size and focused to a waist position in the middle of the full C100 cryomodule allowing the positron beam to make it till the end of C100. Nevertheless, a small number of positrons at large angles still hit the cryomodules during this.

In Table 13, the efficiency, equivalent current with a $10 \mathrm{~mA}$ incoming electron beam, the positron momentum, the geometrical transverse emittance $\varepsilon_{x y}$ and time spread of the positrons at different BPM locations are given. This table was prepared by using G4 tracking results. At the end of the lattice, the positrons distributions 


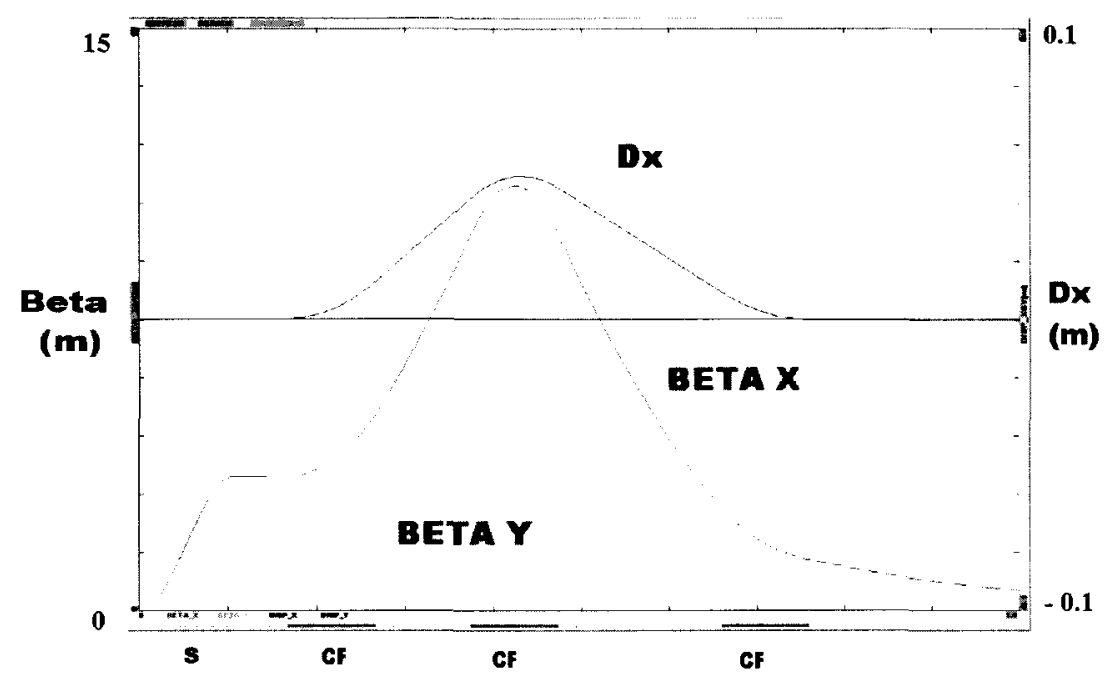

FIG. 54: This plot zooms into the first few meters of the CFM lattice. Only CFM magnets and solenoid elements are shown from OPTIM. BetaX and BetaY are twiss $\beta \mathrm{s}$ and $\mathrm{Dx}$ is the dispersion function. 'S' at the beginning of the lattice is the Solenoid, 'CF' is the Comined Function magnets.

have outliers, which were biasing the emittance and time \& momentum values. As a result of that, a recalculation of the r.m.s values was completed by collimating (hard cut) the beam transverse parameters at $2.5 \sigma_{\text {old }}$. The new r.m.s values give better numbers while it is inevitable to lose a portion of the beam current as provided in Table 13. At the end of the CFM configuration, there is $\sim 3 \mu \mathrm{A}$ of positron current within the admittance values of CEBAF with the assumptions of $10 \mathrm{~mA} 126 \mathrm{MeV}$ electron beam hitting a $2 \mathrm{~mm}$ tungsten with $\sigma=100 \mu \mathrm{m}$ spot size. In Fig. 57, the $p, t, x, y$ and transverse phase space areas at the connection point of North Linac (at BPM \# iIPM11) are plotted with and without outliers. The last row of Table 13 was prepared by using the recalculated r.m.s values from these plots. The full lattice design parameters such as $s$ positions, length and field values of the magnetic elements as well as the twiss parameters at the end of these elements are provided in Table 14. 


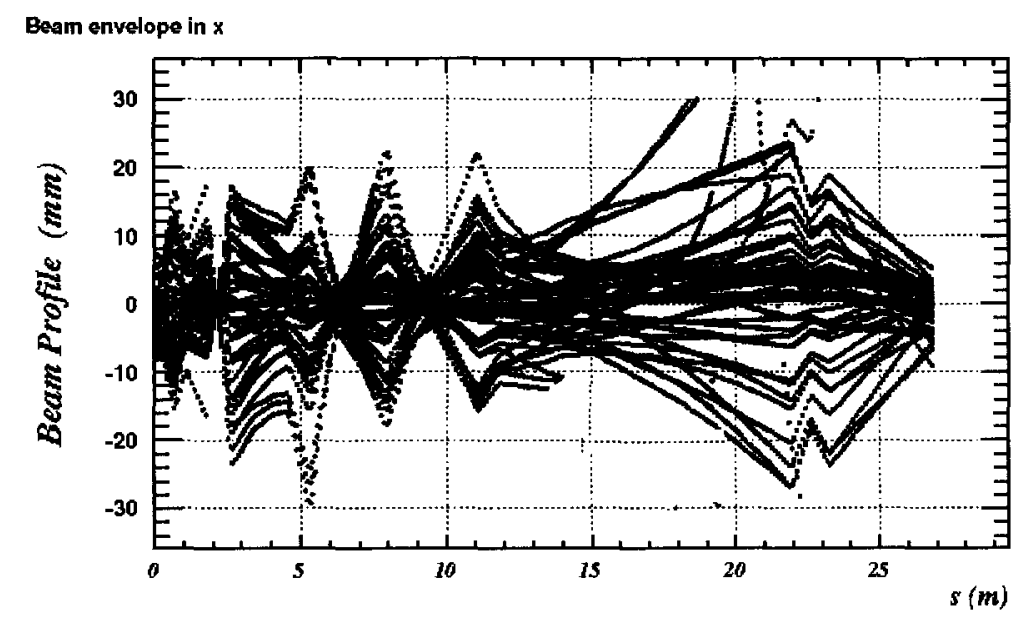

FIG. 55: The full beam envelope of the cut positron beam in transverse $x$. This is the simulation result from the G4 propagated through the CFM lattice.

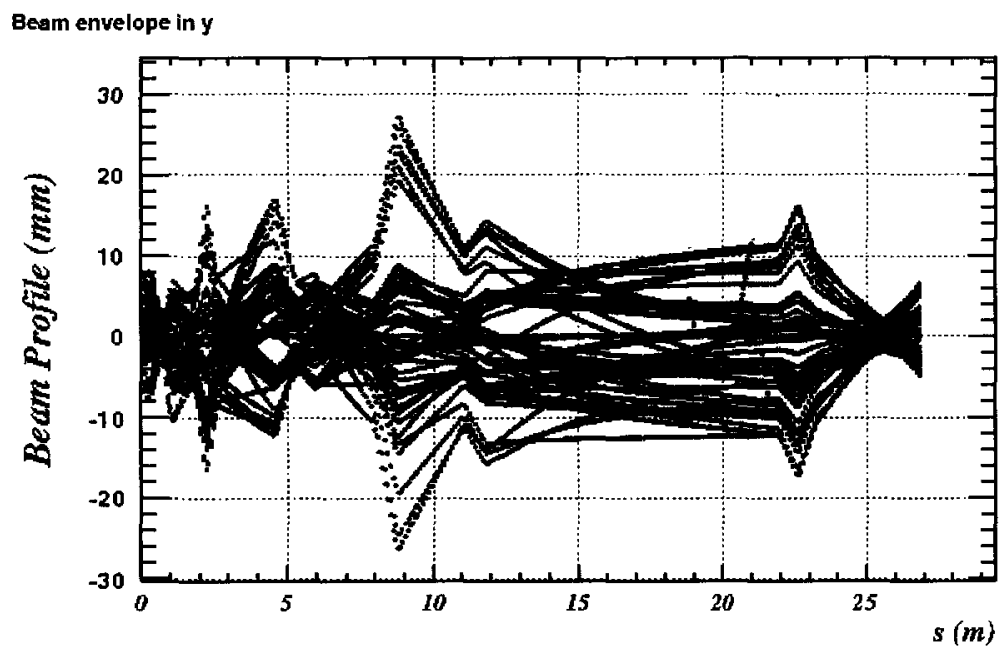

FIG. 56: The full beam envelope of the cut positron beam in transverse $y$. This is the simulation result from the G4 propagated through the CFM lattice. 
TABLE 13: The efficiency and equivalent current at $10 \mathrm{~mA}$ incoming electron beam, momentum, geometrical emittance and time spread information of the positron beam for the CFM lattice at different BPMs in the lattice. In this table these values are obtained by propagating the cut positrons from G4beamline simulation code. The output data to create this table is filtered via a cut to trim the outliers. The outliers were about $15 \%$.

\begin{tabular}{ccccccc}
\hline \hline Detector & $\begin{array}{c}\text { Efficiency } \\
\left(\frac{\mathrm{e}^{+}}{e^{-}} 10^{-4}\right)\end{array}$ & $\begin{array}{c}\text { Current } \\
(\mu A)\end{array}$ & $\begin{array}{c}p \pm \sigma_{p} \\
(\mathrm{MeV} / \mathrm{c})\end{array}$ & $\begin{array}{c}\varepsilon_{X} \\
(\mathrm{~mm} \cdot \mathrm{mrad})\end{array}$ & $\begin{array}{c}\varepsilon_{Y} \\
(\mathrm{~mm} \cdot \mathrm{mrad})\end{array}$ & $\begin{array}{c}\sigma_{t} \\
(\mathrm{ps})\end{array}$ \\
\hline Source & 3.6 & 3.6 & $15.0 \pm 0.5$ & 5.3 & 5.3 & 0.1 \\
iIPM0 & 3.6 & 3.6 & $15.0 \pm 0.5$ & 13.3 & 13.3 & 0.74 \\
iIPM5 & 3.6 & 3.6 & $25.0 \pm 0.5$ & 10.0 & 10.0 & 1.7 \\
IIPM7 & 3.6 & 3.6 & $35.0 \pm 0.5$ & 5.5 & 5.7 & 2.1 \\
iIPM11 & 2.9 & 2.9 & $126.0 \pm 0.9$ & 1.6 & 1.7 & 1.8 \\
\hline \hline
\end{tabular}




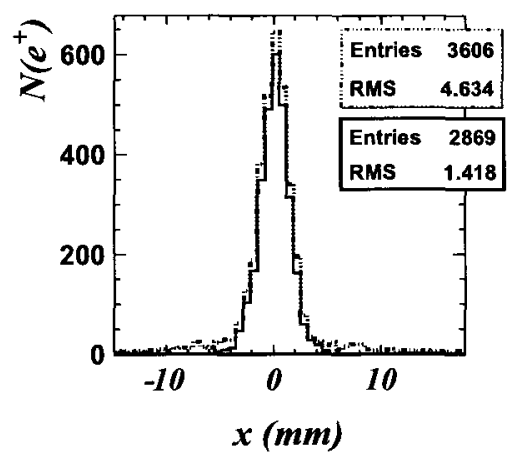

(a)

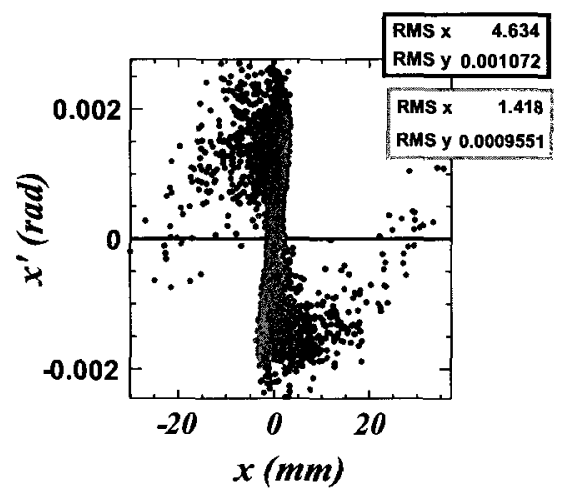

(c)

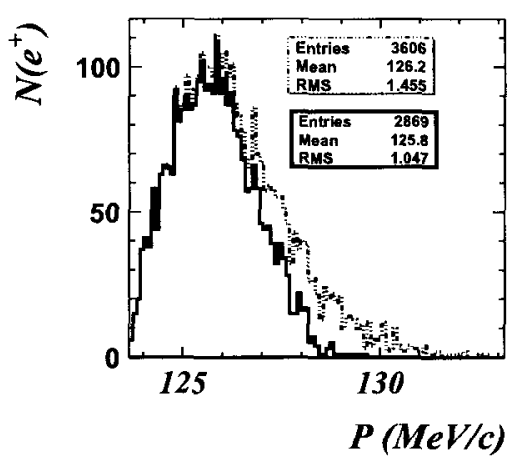

(e)

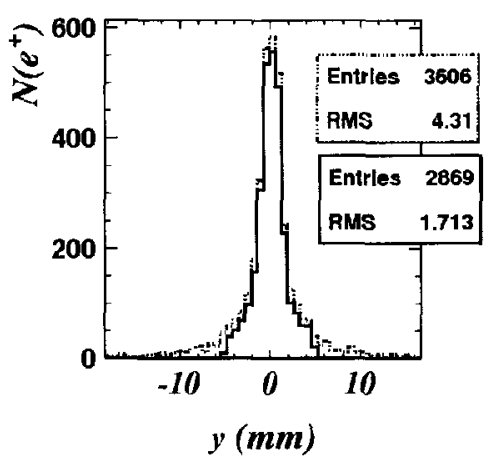

(b)

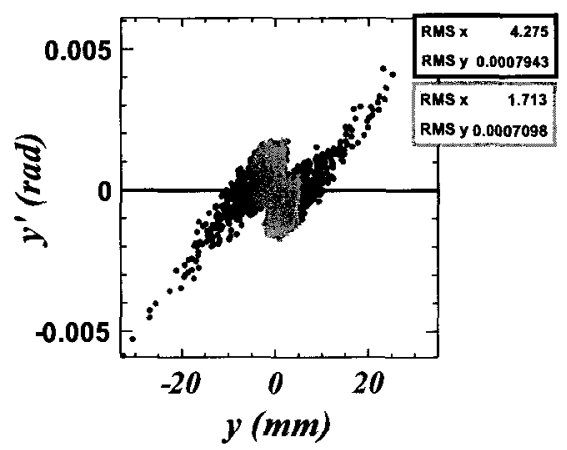

(d)

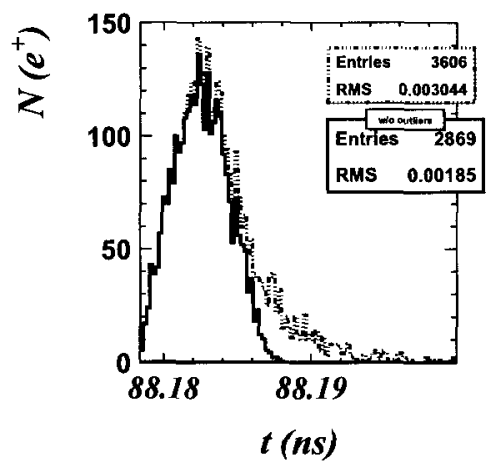

(f)

FIG. 57: The positron beam at the end of the CFM lattice. In 1D plots, the dashed line shows the selected $\mathrm{e}^{+}$beam, which reaches to the North Linac connection point. These $\mathrm{e}^{+}$have outliers extending far from the mean values. So recalculation of the r.m.s values were necessary. This was completed by putting a collimator (hard cut) at $2.5 \mathrm{r} . \mathrm{m} . \mathrm{s}_{\text {old }}$, and recalculate the r.m.s $\mathrm{s}_{\text {new }}$ values accordingly. The solid lines in the figures show the recalculated r.m.s values. In $2 \mathrm{D}$ plots transverse phase space are shown in (c) and (d) where the darker area is the positrons with outliers and lighter area is without outliers. These plots are tracking results from G4BEAMLINE simulation. 
TABLE 14: Lattice description of the Combined Function Magnet configuration. The dipole field values of the CFMs are: $\mathrm{BqA} 1=0.5523298 k G, \mathrm{BqA} 2=$ $-0.503222 k G, \mathrm{BqA} 3=0.4015302 k G$. Solenoid $\mathrm{C} 01$ has a field $\mathrm{B}=9.8 \mathrm{kG}$. Initial geometrical emittances given as input are $\varepsilon_{x y}=5.8 \mathrm{~mm} \cdot \mathrm{mrad}$ at $p\left(e^{+}\right)=15 \mathrm{MeV} / \mathrm{c}$.

\begin{tabular}{|c|c|c|c|c|c|c|c|c|c|}
\hline \hline N & Name & Type & $\begin{array}{c}\mathbf{S} \\
{[\mathbf{c m}]}\end{array}$ & $\begin{array}{c}\mathbf{L} \\
{[\mathbf{c m}]}\end{array}$ & $\begin{array}{c}\mathbf{G} \\
{[\mathbf{k G} / \mathbf{c m}]}\end{array}$ & $\begin{array}{c}\beta_{x} \\
{[\mathbf{c m}]}\end{array}$ & $\begin{array}{c}\alpha_{x} \\
-\end{array}$ & $\begin{array}{c}\beta_{y} \\
{[\mathbf{c m}]}\end{array}$ & $\begin{array}{c}\alpha_{y} \\
-\end{array}$ \\
\hline \hline 0 & START & & 0 & & & 0.3 & -0.1 & 0.3 & -0.1 \\
\hline 1 & C01 & Solenoid & 16 & 16 & & 344 & -0.1 & 336 & -0.1 \\
\hline 2 & oDAB0 & Drift & 22 & 6 & & 344 & -0.1 & 337 & -0.1 \\
\hline 3 & HC & Collimator & 22 & 0 & & 344 & -0.1 & 337 & -0.1 \\
\hline 4 & oD00 & Drift & 25 & 3 & & 345 & -0.1 & 338 & -0.1 \\
\hline 5 & iIPM0 & BPM & 25 & 0 & & 345 & -0.1 & 338 & -0.1 \\
\hline 6 & oD00 & Drift & 28 & 3 & & 345 & -0.1 & 338 & -0.1 \\
\hline 7 & BqA1 & CFM & 43 & 15 & -0.055 & 430 & -6.0 & 264 & 4.6 \\
\hline 8 & oA00 & Drift & 46 & 3 & & 467 & -6.3 & 238 & 4.4 \\
\hline 9 & iIPM1 & BPM & 46 & 0 & & 467 & -6.3 & 238 & 4.4 \\
\hline 10 & oAB1 & Drift & 58 & 12 & & 630 & -7.3 & 145 & 3.3 \\
\hline 11 & HC & Collimator & 58 & 0 & & 630 & -7.3 & 145 & 3.3 \\
\hline 12 & oA00 & Drift & 61 & 3 & & 675 & -7.6 & 126 & 3.1 \\
\hline 13 & BqA2 & CFM & 76 & 15 & 0.095 & 596 & 12.0 & 88 & -0.2 \\
\hline 14 & oA00 & Drift & 79 & 3 & & 526 & 11.3 & 89 & -0.2 \\
\hline 15 & iIPM2 & BPM & 79 & 0 & & 526 & 11.3 & 89 & -0.2 \\
\hline 16 & oAB2 & Drift & 103 & 24 & & 124 & 5.4 & 108 & -0.5 \\
\hline 17 & oA00 & Drift & 106 & 3 & & 94 & 4.7 & 111 & -0.6 \\
\hline 18 & HC & Collimator & 106 & 0 & & 94 & 4.7 & 111 & -0.6 \\
\hline 19 & BqA3 & CFM & 121 & 15 & -0.07 & 15 & 1.1 & 95 & 1.5 \\
\hline 20 & oA00 & Drift & 124 & 3 & & 10 & 0.6 & 87 & 1.4 \\
\hline 21 & iIPM3 & BPM & 124 & 0 & & 10 & 0.6 & 87 & 1.4 \\
\hline 22 & oAB3 & Drift & 184 & 60 & & 435 & -7.7 & 42 & -0.6 \\
\hline & & & Continued on Next Page.. & & & \\
\hline
\end{tabular}


TABLE 14 - Continued

\begin{tabular}{|c|c|c|c|c|c|c|c|c|c|}
\hline $\mathbf{N}$ & Name & Type & $\begin{array}{c}\mathrm{S} \\
{[\mathrm{cm}]}\end{array}$ & $\begin{array}{c}\mathrm{L} \\
{[\mathrm{cm}]}\end{array}$ & $\begin{array}{c}\mathrm{G} \\
{[\mathrm{kG} / \mathrm{cm}]}\end{array}$ & $\begin{array}{c}\beta_{x} \\
{[\mathrm{~cm}]}\end{array}$ & $\alpha_{x}$ & $\begin{array}{c}\beta_{y} \\
{[\mathbf{c m}]}\end{array}$ & $\begin{array}{c}\alpha_{y} \\
-\end{array}$ \\
\hline 23 & $\mathrm{HC}$ & Collimator & 184 & 0 & & 435 & -7.7 & 42 & -0.6 \\
\hline 24 & $\mathrm{oA} 00$ & Drift & 187 & 3 & & 483 & -8.2 & 46 & -0.7 \\
\hline 25 & qA1 & Quad & 202 & 15 & 0.153 & 392 & 12.7 & 128 & -5.9 \\
\hline 26 & oDA1 & Drift & 222 & 20 & & 49 & 4.4 & 478 & -11.6 \\
\hline 27 & qA2 & Quad & 237 & 15 & -0.129 & 7 & -1.1 & 531 & 8.8 \\
\hline 28 & oDA2 & Drift & 262 & 25 & & 256 & -8.8 & 184 & 5.1 \\
\hline 29 & qA3 & Quad & 277 & 15 & 0.107 & 399 & 0.9 & 119 & -0.1 \\
\hline 30 & oDA3 & Drift & 297 & 20 & & 364 & 0.8 & 128 & -0.3 \\
\hline 31 & iIPM4 & $\mathrm{BPM}$ & 297 & 0 & & 364 & 0.8 & 128 & -0.3 \\
\hline 32 & $\mathrm{o} 1006$ & Drift & 312 & 15 & & 341 & 0.7 & 138 & -0.4 \\
\hline 33 & $\mathrm{aC} 50-1$ & RF & 362 & 50 & & 268 & 0.7 & 195 & -0.7 \\
\hline 34 & 01006 & Drift & 377 & 15 & & 248 & 0.6 & 218 & -0.8 \\
\hline 35 & $\mathrm{aC} 50-1$ & $\mathrm{RF}$ & 427 & 50 & & 198 & 0.4 & 316 & -1.1 \\
\hline 36 & o1006 & Drift & 442 & 15 & & 187 & 0.3 & 351 & -1.2 \\
\hline 37 & iIPM5 & BPM & 442 & 0 & & 187 & 0.3 & 351 & -1.2 \\
\hline 38 & oDB0 & Drift & 462 & 20 & & 177 & 0.2 & 402 & -1.4 \\
\hline 39 & qB1 & Quad & 477 & 15 & -0.05 & 197 & -1.6 & 389 & 2.2 \\
\hline 40 & oDB1 & Drift & 534 & 57 & & 435 & -2.6 & 188 & 1.3 \\
\hline 41 & qB2 & Quad & 549 & 15 & 0.09 & 408 & 4.3 & 193 & -1.8 \\
\hline 42 & oDB2 & Drift & 596 & 47 & & 109 & 2.1 & 406 & -2.8 \\
\hline 43 & qB3 & Quad & 611 & 15 & -0.065 & 72 & 0.6 & 417 & 2.1 \\
\hline 44 & oDB3 & Drift & 632 & 21 & & 56 & 0.2 & 335 & 1.8 \\
\hline 45 & iIPM6 & BPM & 632 & 0 & & 56 & 0.2 & 335 & 1.8 \\
\hline 46 & o1006 & Drift & 647 & 15 & & 54 & -0.1 & 284 & 1.6 \\
\hline 47 & $\mathrm{aC} 50-2$ & $\mathrm{RF}$ & 697 & 50 & & 109 & -1.0 & 151 & 1.0 \\
\hline 48 & o1006 & Drift & 712 & 15 & & 143 & -1.3 & 124 & 0.8 \\
\hline 49 & aC50-2 & $\mathrm{RF}$ & 762 & 50 & & 313 & -2.1 & 75 & 0.2 \\
\hline 50 & 01006 & Drift & 777 & 15 & & 382 & -2.4 & 74 & 0.0 \\
\hline 51 & iIPM7 & $\mathrm{BPM}$ & 777 & 0 & & 382 & -2.4 & 74 & 0.0 \\
\hline \multicolumn{10}{|c|}{ Continued on Next Page. . } \\
\hline
\end{tabular}


TABLE 14 - Continued

\begin{tabular}{|c|c|c|c|c|c|c|c|c|c|}
\hline $\mathbf{N}$ & Name & Type & $\begin{array}{c}\mathrm{S} \\
{[\mathrm{cm}]}\end{array}$ & $\begin{array}{c}\mathbf{L} \\
{[\mathrm{cm}]}\end{array}$ & $\begin{array}{c}\mathbf{G} \\
{[\mathrm{kG} / \mathrm{cm}]}\end{array}$ & $\begin{array}{c}\beta_{x} \\
{[\mathbf{c m}]}\end{array}$ & $\begin{array}{r}\alpha_{x} \\
-\end{array}$ & $\begin{array}{c}\beta_{y} \\
{[\mathbf{c m}]}\end{array}$ & $\begin{array}{c}\alpha_{y} \\
- \\
\end{array}$ \\
\hline 52 & oDC0 & Drift & 797 & 20 & & 485 & -2.8 & 81 & -0.3 \\
\hline 53 & $\mathrm{qC} 1$ & Quad & 812 & 15 & 0.12 & 455 & 4.6 & 116 & -2.2 \\
\hline 54 & oDC1 & Drift & 878 & 66 & & 62 & 1.4 & 617 & -5.4 \\
\hline 55 & $\mathrm{qC} 2$ & Quad & 893 & 15 & -0.07 & 36 & 0.4 & 697 & 0.4 \\
\hline 56 & oDC2 & Drift & 937 & 44 & & 63 & -1.0 & 668 & 0.3 \\
\hline 57 & $\mathrm{qC} 3$ & Quad & 952 & 15 & -0.01 & 103 & -1.6 & 647 & 1.1 \\
\hline 58 & oDC3 & Drift & 1106 & 154 & & 1460 & -7.2 & 389 & 0.6 \\
\hline 59 & iIPM8 & $\mathrm{BPM}$ & 1106 & 0 & & 1460 & -7.2 & 389 & 0.6 \\
\hline 60 & oD00 & Drift & 1109 & 3 & & 1504 & -7.3 & 385 & 0.6 \\
\hline 61 & $\mathrm{qD} 1$ & Quad & 1124 & 15 & 0.075 & 1502 & 7.4 & 426 & -3.4 \\
\hline 62 & oDD1 & Drift & 1182 & 58 & & 772 & 5.2 & 919 & -5.1 \\
\hline 63 & qD2 & Quad & 1197 & 15 & -0.05 & 690 & 0.4 & 983 & 1.0 \\
\hline 64 & oDD2 & Drift & 1317 & 120 & & 616 & 0.2 & 781 & 0.7 \\
\hline 65 & ilPM9 & $\mathrm{BPM}$ & 1317 & 0 & & 616 & 0.2 & 781 & 0.7 \\
\hline 66 & o1005 & Drift & 1342 & 25 & & 607 & 0.2 & 746 & 0.7 \\
\hline 67 & o1006 & Drift & 1357 & 15 & & 603 & 0.1 & 726 & 0.6 \\
\hline 68 & $\mathrm{aC} 100$ & $\mathrm{RF}$ & 1427 & 70 & & 573 & 0.3 & 624 & 0.8 \\
\hline 69 & o1006 & Drift & 1442 & 15 & & 565 & 0.2 & 601 & 0.7 \\
\hline 70 & 01006 & Drift & 1457 & 15 & & 559 & 0.2 & 579 & 0.7 \\
\hline 71 & $\mathrm{aC} 100$ & $\mathrm{RF}$ & 1527 & 70 & & 529 & 0.2 & 484 & 0.6 \\
\hline 72 & o1006 & Drift & 1542 & 15 & & 523 & 0.2 & 465 & 0.6 \\
\hline 73 & o1006 & Drift & 1557 & 15 & & 518 & 0.2 & 448 & 0.6 \\
\hline 74 & $\mathrm{aC} 100$ & $\mathrm{RF}$ & 1627 & 70 & & 500 & 0.1 & 380 & 0.4 \\
\hline 75 & o1006 & Drift & 1642 & 15 & & 497 & 0.1 & 368 & 0.4 \\
\hline 76 & o1006 & Drift & 1657 & 15 & & 496 & 0.0 & 357 & 0.3 \\
\hline 77 & $\mathrm{aC} 100$ & $\mathrm{RF}$ & 1727 & 70 & & 496 & 0.0 & 324 & 0.1 \\
\hline 78 & o1006 & Drift & 1742 & 15 & & 498 & -0.1 & 321 & 0.1 \\
\hline 79 & o1006 & Drift & 1757 & 15 & & 500 & -0.1 & 318 & 0.1 \\
\hline 80 & $\mathrm{aC} 100$ & RF & 1827 & 70 & & 521 & -0.2 & 324 & -0.1 \\
\hline \multicolumn{10}{|c|}{ Continued on Next Page... } \\
\hline
\end{tabular}


TABLE 14 - Continued

\begin{tabular}{|c|c|c|c|c|c|c|c|c|c|}
\hline \hline N & Name & Type & $\begin{array}{c}\mathbf{S} \\
{[\mathbf{c m}]}\end{array}$ & $\begin{array}{c}\mathbf{L} \\
{[\mathbf{c m}]}\end{array}$ & $\begin{array}{c}\mathbf{G} \\
{[\mathbf{k G} / \mathbf{c m}]}\end{array}$ & $\begin{array}{c}\beta_{x} \\
{[\mathbf{c m}]}\end{array}$ & $\begin{array}{c}\alpha_{x} \\
-\end{array}$ & $\begin{array}{c}\beta_{y} \\
{[\mathbf{c m}]}\end{array}$ & $\begin{array}{c}\alpha_{y} \\
-\end{array}$ \\
\hline \hline 81 & o1006 & Drift & 1842 & 15 & & 527 & -0.2 & 329 & -0.2 \\
\hline 82 & o1006 & Drift & 1857 & 15 & & 534 & -0.3 & 336 & -0.2 \\
\hline 83 & aC100 & RF & 1927 & 70 & & 577 & -0.4 & 382 & -0.4 \\
\hline 84 & o1006 & Drift & 1942 & 15 & & 588 & -0.4 & 395 & -0.5 \\
\hline 85 & o1006 & Drift & 1957 & 15 & & 600 & -0.4 & 410 & -0.5 \\
\hline 86 & aC100 & RF & 2027 & 70 & & 665 & -0.5 & 496 & -0.7 \\
\hline 87 & o1006 & Drift & 2042 & 15 & & 680 & -0.5 & 518 & -0.8 \\
\hline 88 & o1006 & Drift & 2057 & 15 & & 697 & -0.6 & 541 & -0.8 \\
\hline 89 & aC100 & RF & 2127 & 70 & & 783 & -0.7 & 665 & -1.0 \\
\hline 90 & o1006 & Drift & 2142 & 15 & & 803 & -0.7 & 695 & -1.0 \\
\hline 91 & o1008 & Drift & 2167 & 25 & & 839 & -0.7 & 748 & -1.1 \\
\hline 92 & iIPM10 & BPM & 2167 & 0 & & 839 & -0.7 & 748 & -1.1 \\
\hline 93 & oDE0 & Drift & 2197 & 30 & & 885 & -0.8 & 817 & -1.2 \\
\hline 94 & qE1 & Quad & 2212 & 15 & -0.134 & 975 & -5.4 & 794 & 2.6 \\
\hline 95 & oDE1 & Drift & 2262 & 50 & & 1586 & -6.9 & 554 & 2.1 \\
\hline 96 & qE2 & Quad & 2277 & 15 & 0.278 & 1555 & 8.9 & 573 & -3.4 \\
\hline 97 & oDE2 & Drift & 2327 & 50 & & 795 & 6.3 & 970 & -4.5 \\
\hline 98 & qE3 & Quad & 2342 & 15 & -0.176 & 682 & 1.4 & 1014 & 1.7 \\
\hline 99 & oDE3 & Drift & 2392 & 50 & & 551 & 1.2 & 854 & 1.5 \\
\hline 100 & oD99 & Drift & 2642 & 250 & & 228 & 0.1 & 342 & 0.5 \\
\hline 101 & iIPM11 & BPM & 2642 & 0 & & 228 & 0.1 & 342 & 0.5 \\
\hline 102 & END & & 2642 & & & 228 & 0.1 & 342 & 0.5 \\
\hline \hline & & & & & & & & \\
\hline \hline
\end{tabular}


The phase space distributions at the end of beam position monitors

The following plots from Fig. 58 to $64(\mathrm{~b})$ are the simulation tracking results of G4BEAMLINE. After each CFM, quadrupole triplet, quarter RF and full RF the $x$ $x^{\prime}, y-y^{\prime}, p-t$ and $x-y$ distributions of the positron beams are plotted. These positrons are cut positrons as defined earlier in Sec. IV.1. They are tracked until the end of the lattice.
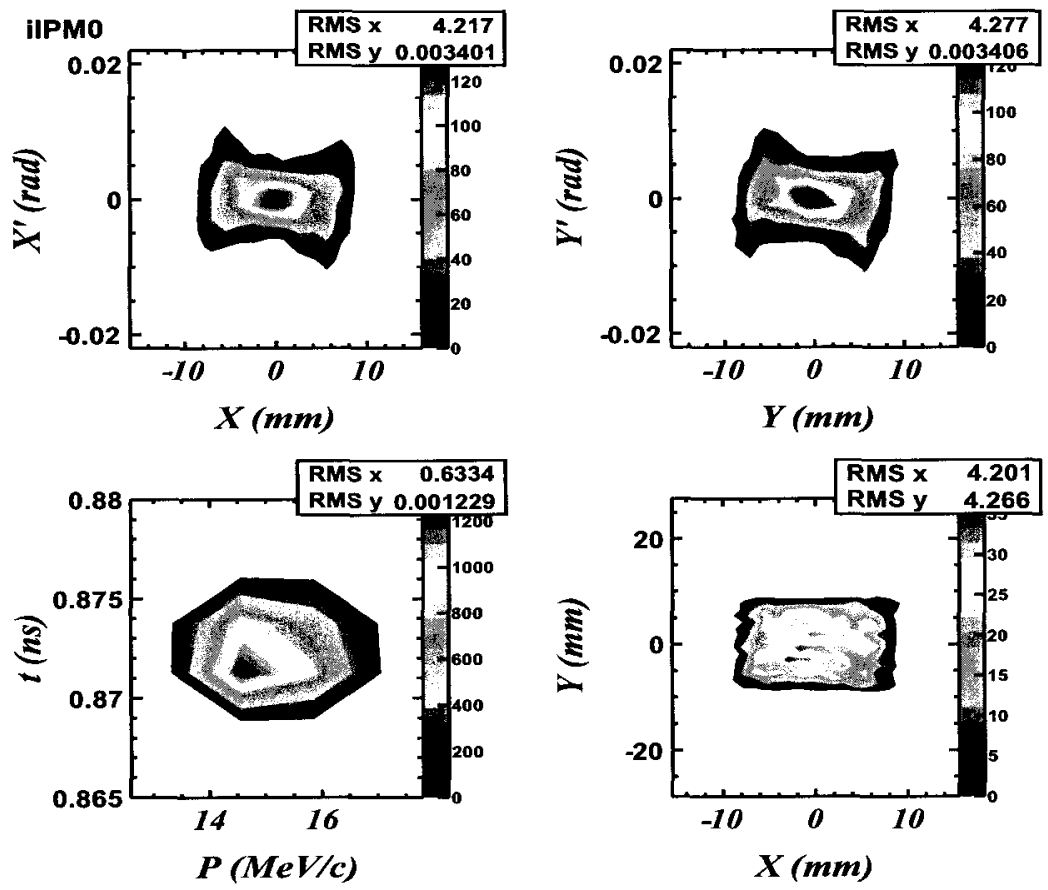

FIG. 58: Transverse and longitudinal phase space of the beam after the solenoid in CFM lattice. $\varepsilon_{x}=13.3 \mathrm{~mm} \cdot \mathrm{mrad} \quad \varepsilon_{y}=13.3 \mathrm{~mm} \cdot \mathrm{mrad}$. 

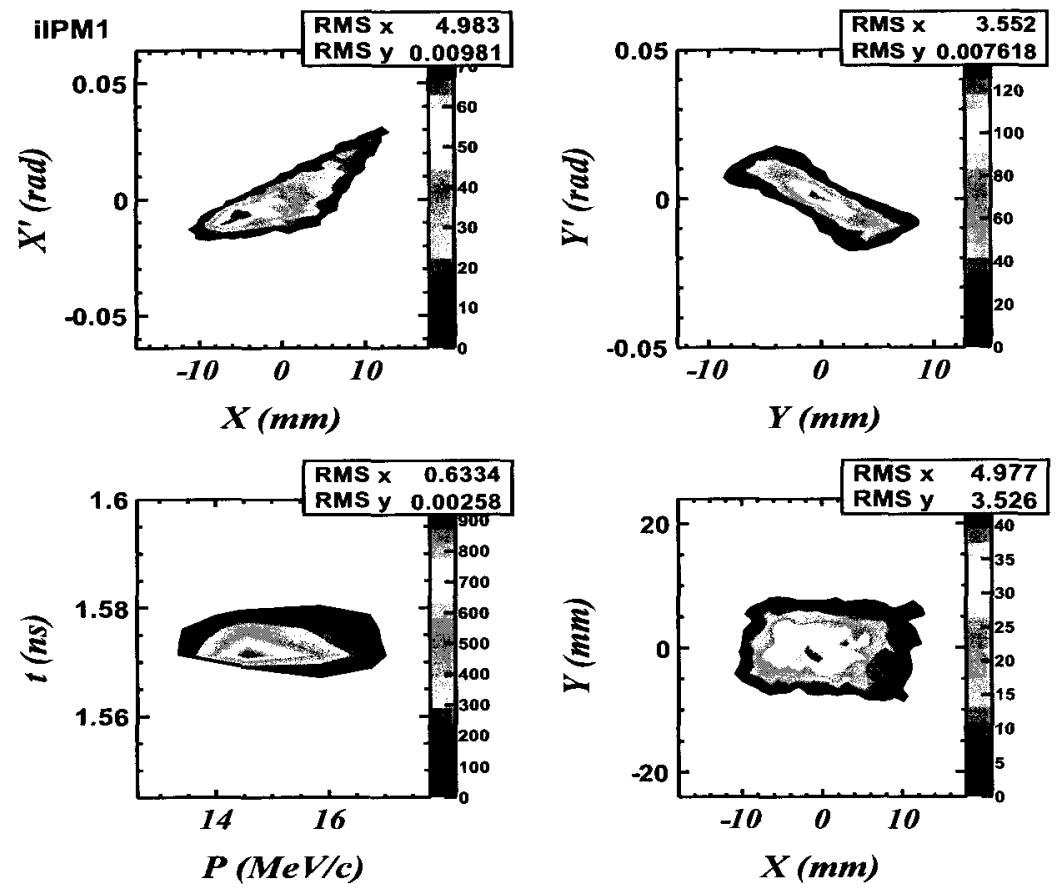

FIG. 59: The $\mathrm{e}^{+}$beam after the 1st CFM magnet in the CFM lattice. $\varepsilon_{x}=25.3$ $\mathrm{mm} \cdot \mathrm{mrad} \quad \varepsilon_{y}=14.3 \mathrm{~mm} \cdot \mathrm{mrad}$. 

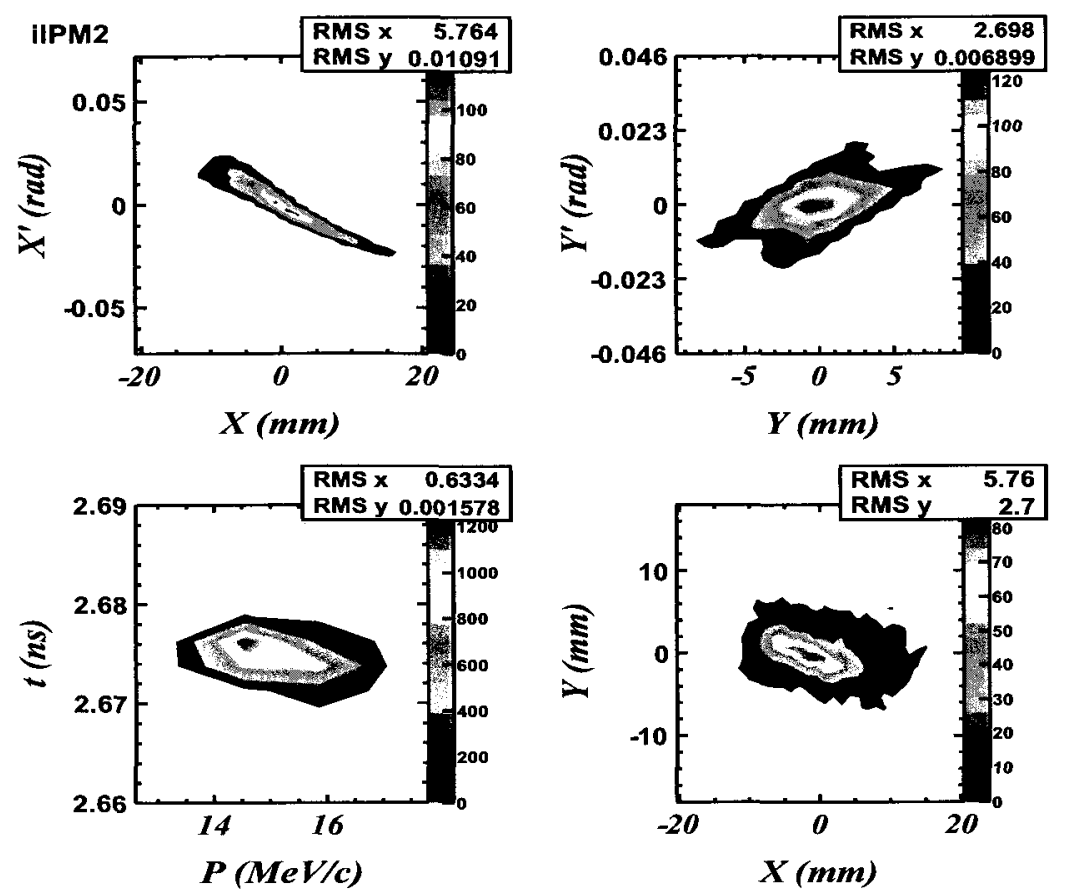

(a)
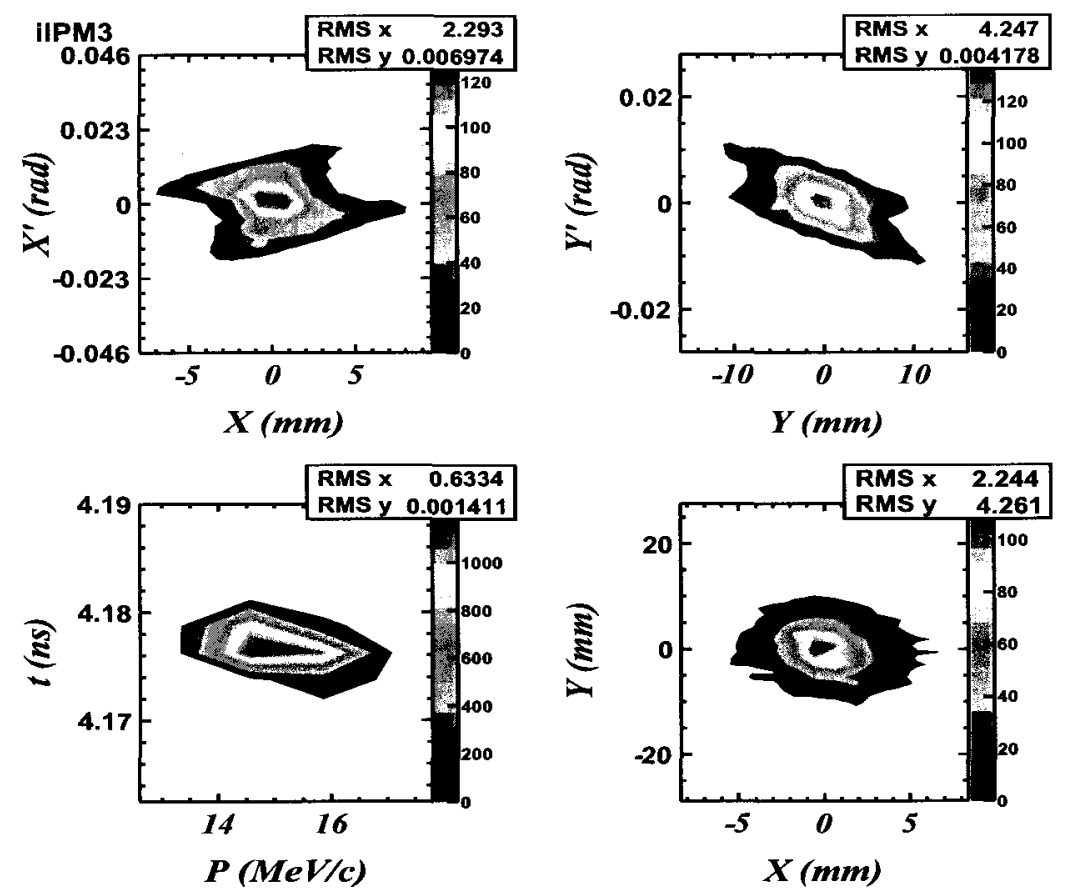

(b)

FIG. 60: (a) Transverse and longitudinal phase space of the beam after the 2nd CFM in CFM lattice. $\varepsilon_{x}=25 \mathrm{~mm} \cdot \mathrm{mrad} \quad \varepsilon_{y}=14 \mathrm{~mm} \cdot \mathrm{mrad}$ (b) After the 3rd CFM in CFM lattice with $\varepsilon_{x}=15 \mathrm{~mm} \cdot \mathrm{mrad} \quad \varepsilon_{y}=15 \mathrm{~mm} \cdot \mathrm{mrad}$ 

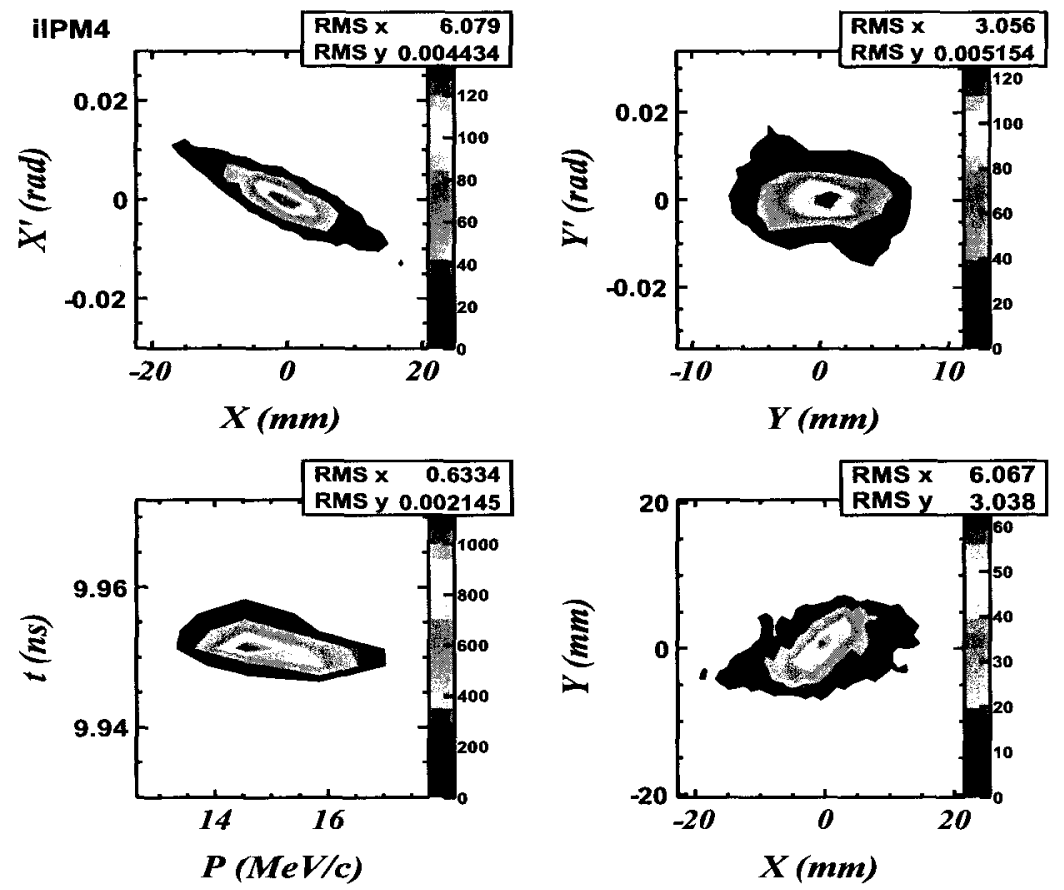

(a)
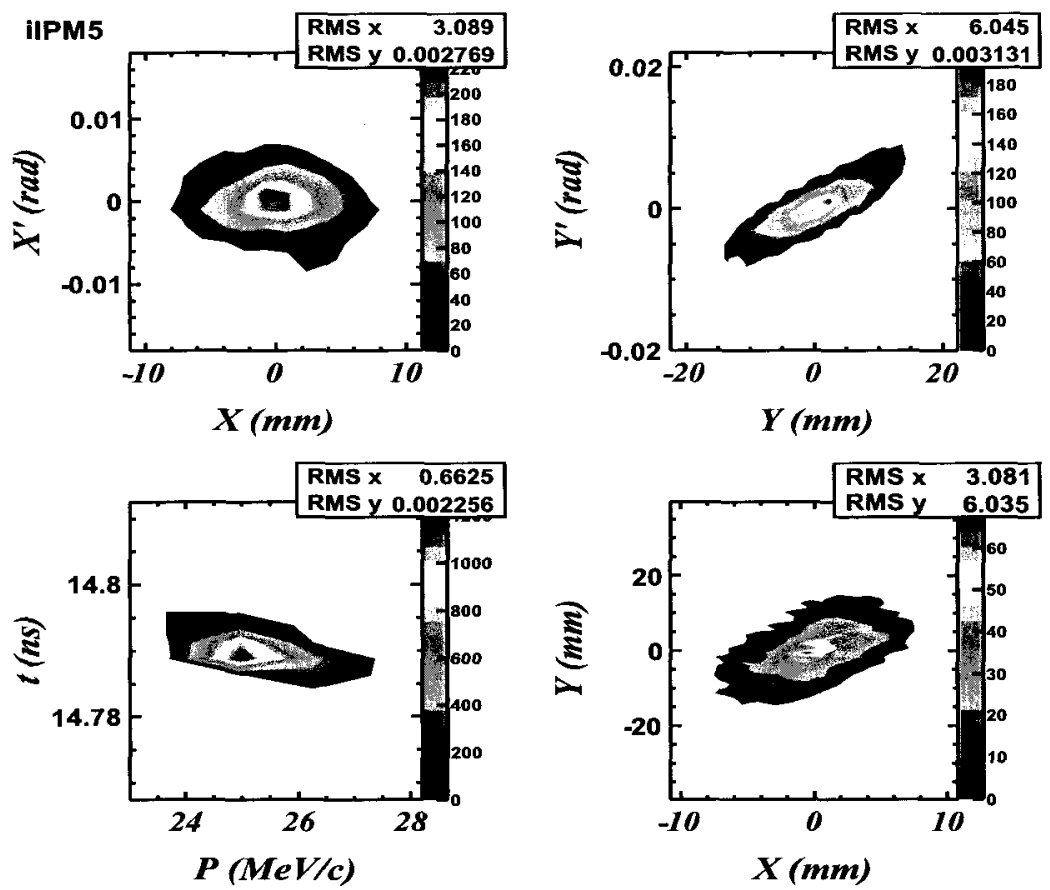

(b)

FIG. 61: (a) Transverse and longitudinal phase space of the beam right before the 1st quarter cryomodule in the CFM lattice. $\varepsilon_{x}=14 \mathrm{~mm} \cdot \mathrm{mrad} \quad \varepsilon_{y}=17 \mathrm{~mm} \cdot \mathrm{mrad}$ (b) After the 1st quarter cryomodule in the CFM lattice. $\varepsilon_{x}=10 \mathrm{~mm} \cdot \mathrm{mrad} \varepsilon_{y}=10$ $\mathrm{mm} \cdot \mathrm{mrad}$ 

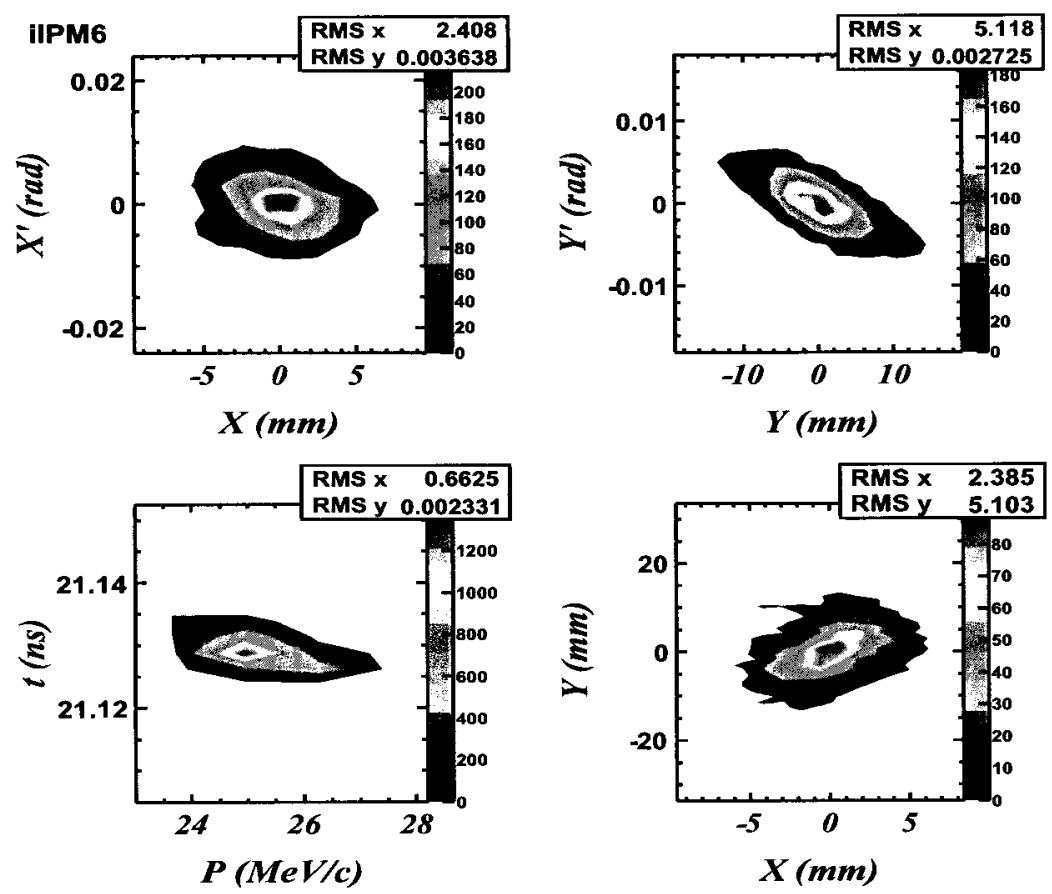

(a)
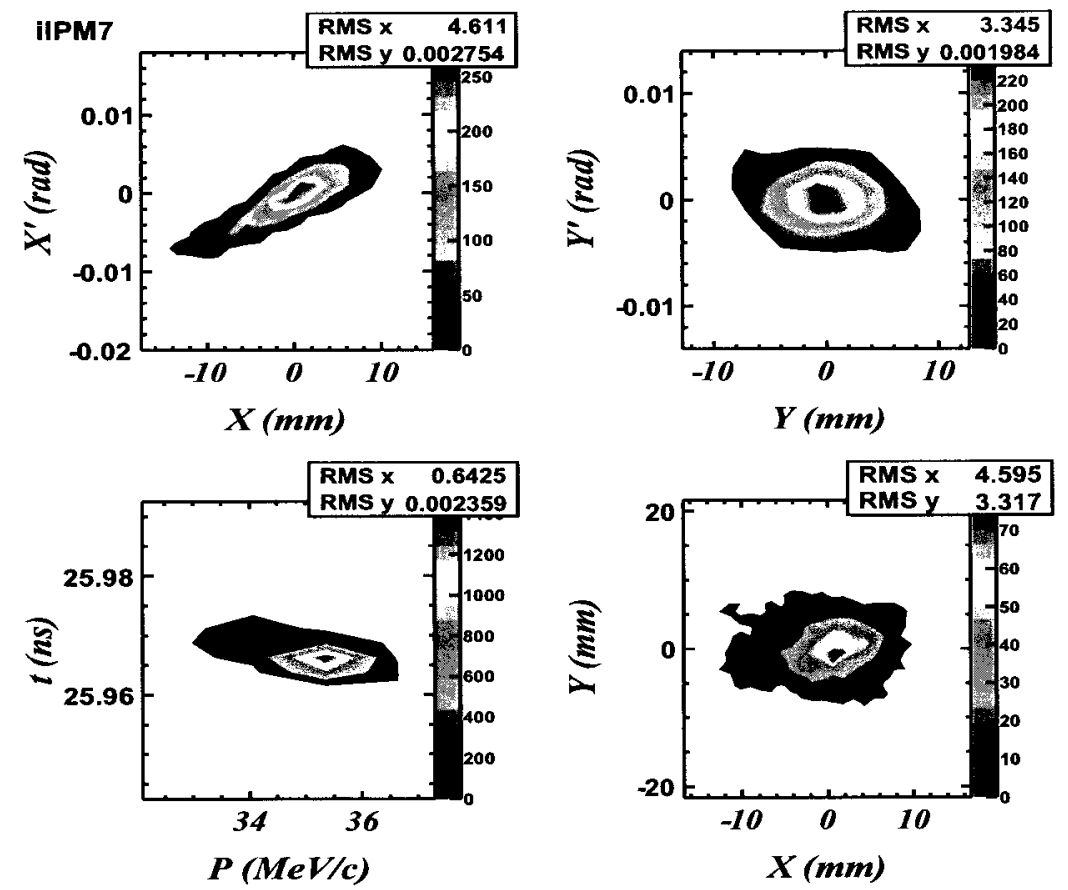

(b)

FIG. 62: (a) Transverse and longitudinal phase space of the beam right before the 2nd quarter cryomodule in the CFM lattice. $\varepsilon_{x}=10 \mathrm{~mm} \cdot \mathrm{mrad} \varepsilon_{y}=10 \mathrm{~mm} \cdot \mathrm{mrad}$ (b) After the 2nd quarter cryomodule in the CFM lattice. $\varepsilon_{x}=5.5 \mathrm{~mm} \cdot \mathrm{mrad}$ $\varepsilon_{y}=5.7 \mathrm{~mm} \cdot \mathrm{mrad}$ 

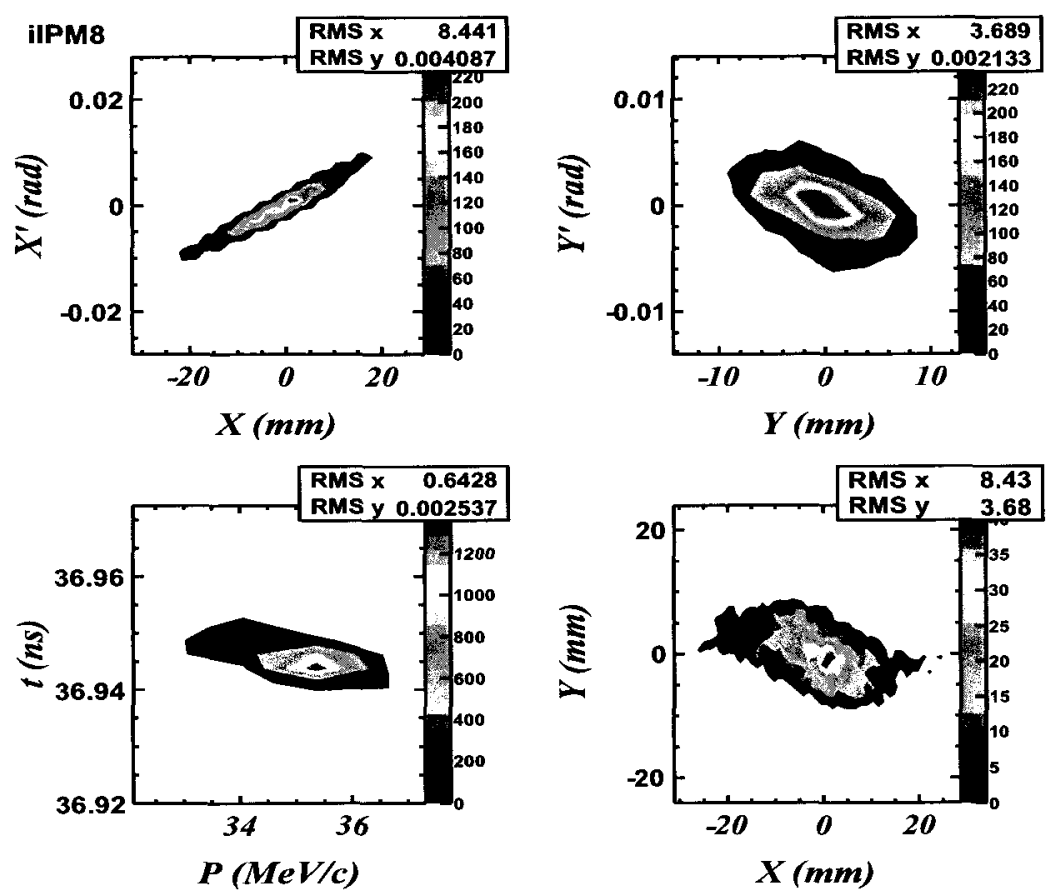

(a)
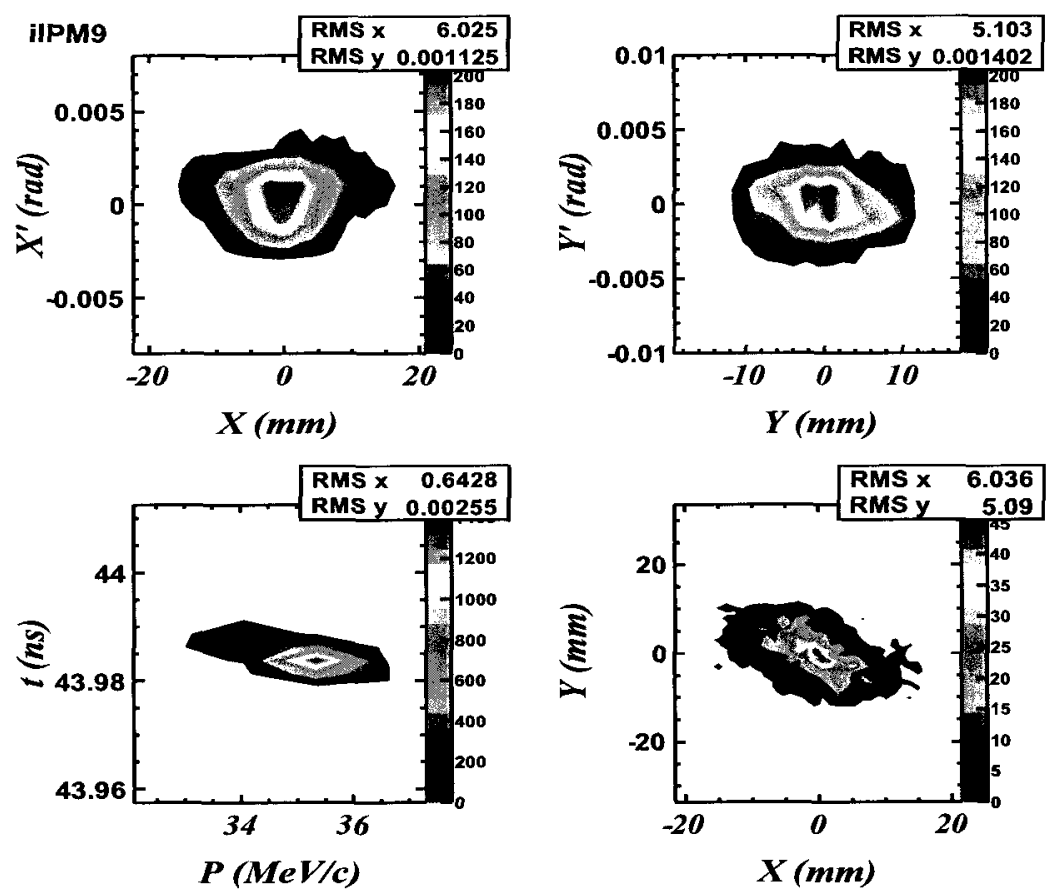

(b)

FIG. 63: (a) Transverse and longitudinal phase space of the beam right after the $\mathrm{qC}$ set quad triplet in the CFM lattice $\varepsilon_{x}=6.7 \mathrm{~mm} \cdot \mathrm{mrad} \quad \varepsilon_{y}=7 \mathrm{~mm} \cdot \mathrm{mrad}$ (b) Right before full cryomodule in the CFM lattice $\varepsilon_{x}=6.7 \mathrm{~mm} \cdot \mathrm{mrad} \varepsilon_{y}=6.5 \mathrm{~mm} \cdot \mathrm{mrad}$ 

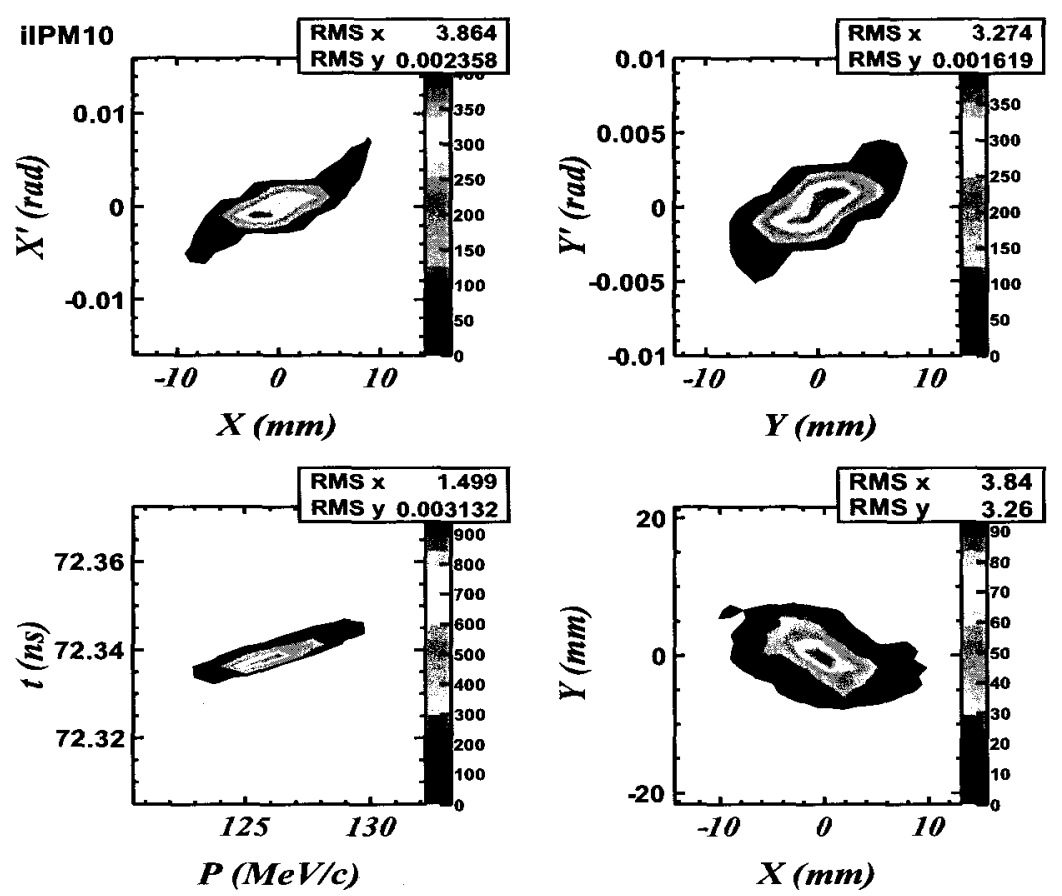

(a)
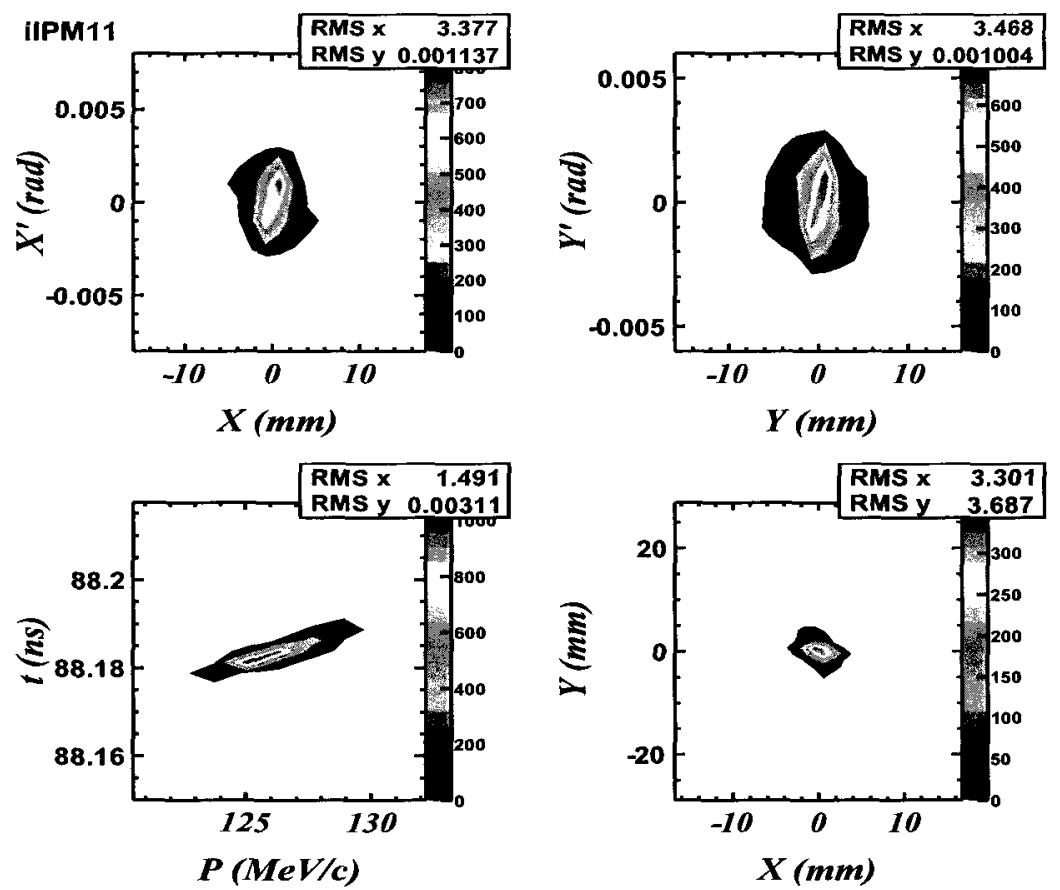

(b)

FIG. 64: (a) Transverse and longitudinal phase space of the beam right after the full cryomodule in the CFM lattice. $\varepsilon_{x}=1.6 \mathrm{~mm} \cdot \mathrm{mrad} \varepsilon_{y}=1.5 \mathrm{~mm} \cdot \mathrm{mrad}(\mathrm{b}) \mathrm{At}$ the end of the lattice where a connection can be made to the North Linac. $\varepsilon_{x}=1.6$ $\mathrm{mm} \cdot \mathrm{mrad} \quad \varepsilon_{y}=1.5 \mathrm{~mm} \cdot \mathrm{mrad}$ 


\section{IV.4 DESIGN - 2 : TWO DIPOLES}

In this 2nd proposed solution, instead of using a combined function magnet triplet, we use a quadrupole triplet right after the QWT solenoid. Then two sector dipoles are positioned after this triplet. Collimators and absorbers are placed in between elements. There must also be a shield covering the surroundings of these front-end elements. In Fig. 65, a simple schematic is shown to illustrate the positions of the elements for this configuration. It is almost the same configuration with CFM after the sector dipole magnets.

In Fig. 66, the G4BEAMLINE snapshot for the proposed Two-Dipole configuration is shown. The red arrow on the left shows the direction of the incoming electron beam. The solenoid captures the positrons (electrons), then immediately after solenoid the quadrupoles transport them to the dipoles for separation. The dipoles kick beams in opposite directions resulting a deviation from the central orbit of an angle $\theta_{1}=10$ degrees, the second dipole gives a kick again in the same direction $\theta_{2}=10$ degrees making the total deviation from the 0-degree line $\theta=20$ degrees for positrons. Since most of the photons are emitted on the 0-degree line, they are mostly attenuated by the collimators. This configuration is different than CFM lattice as the electrons are separated at a later stage in the dipoles, while in the CFM the electrons are separated immediately in the CFM magnets. Simulations show approximately equal amounts of power deposition in the first few elements, but since the electrons are separated at a later stage there may be greater radiation leakage outside of the vault via multiple scatterings of the photons through the beam pipes although not shown in the simulations. 


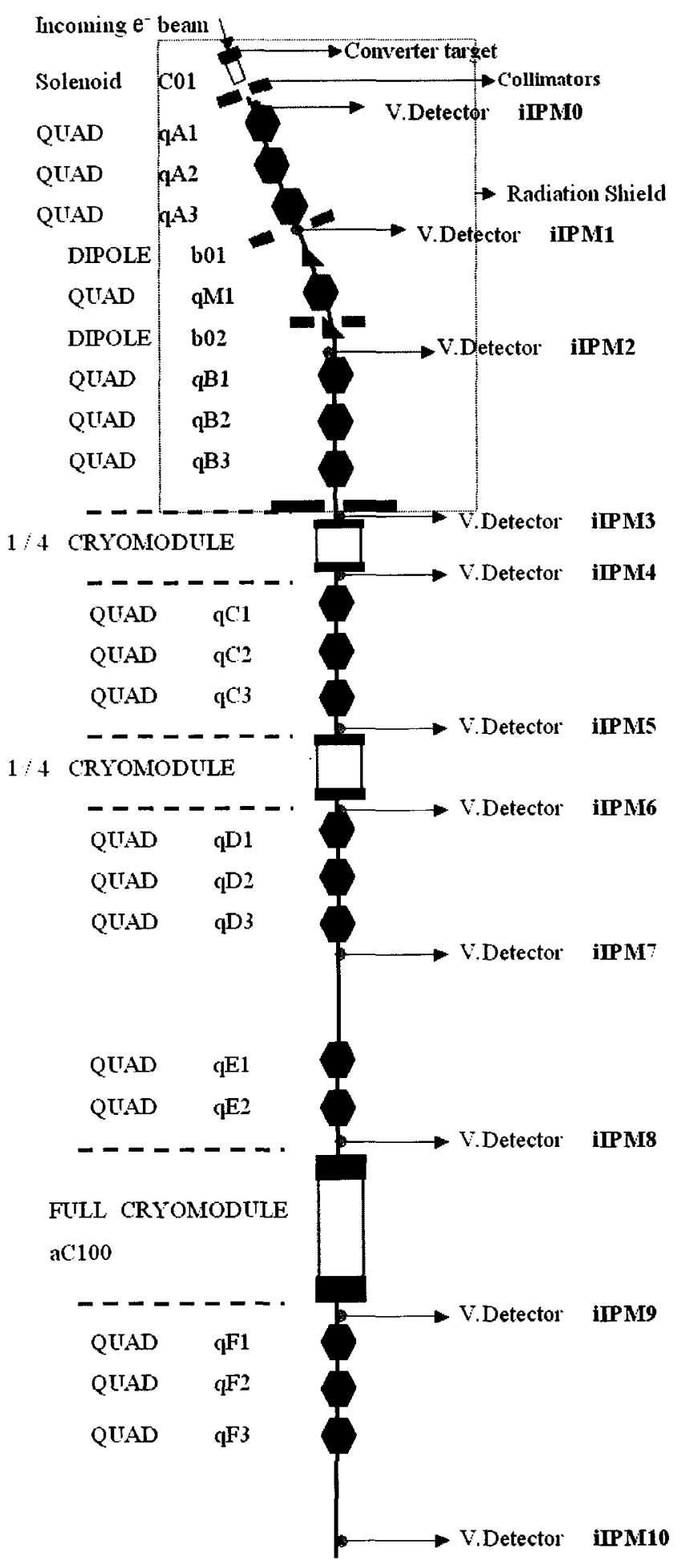

Connection to North Linac

FIG. 65: A quick schematic guide to the Two-Dipole lattice. This is similar but much more simplified copy of the quick guide of the CEBAF injector. 


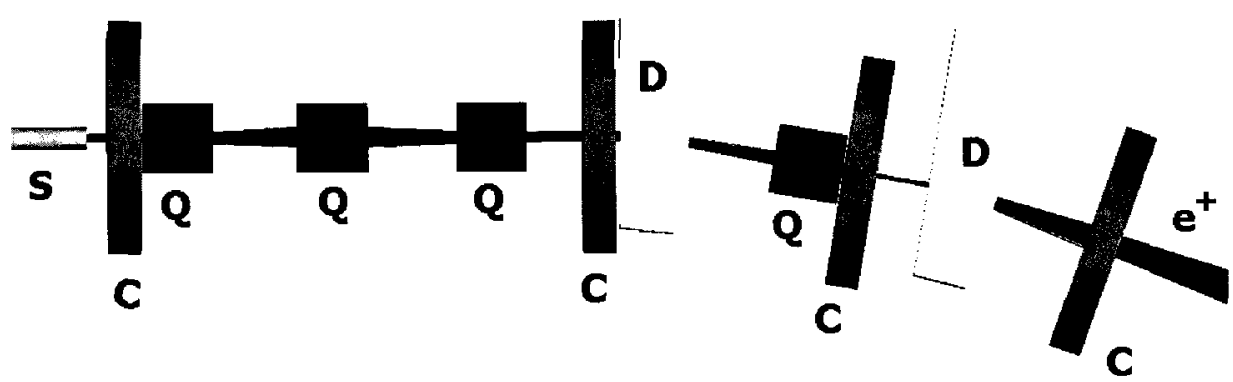

(a)

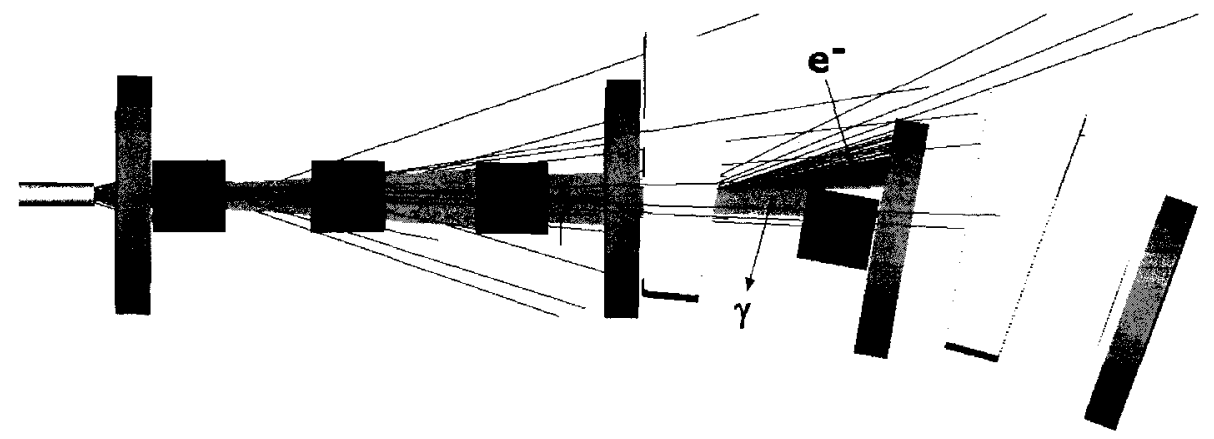

(b)

FIG. 66: A snapshot from the G4BEAMLINE simulation showing the forward secondary particles. The long cylinder on the left is the solenoid (S), quadrupoles are designated with letter (Q), dipoles (D) and the collimators (C) are located in between elements. As the dipole field diverts opposite signed particles in opposite directions, collimators are used to stop electrons. Almost all photons will be stopped as well. (a) Only $\mathrm{e}^{+}$beam is shown, where the beam passes cleanly through the holes of collimators. (b) $\mathrm{e}^{-}$and $\gamma$ are tracked, where the secondary $\mathrm{e}^{-}$are stopped at the first dipole. The cryomodule is secured at a far enough location from the radiation zone. In this figure, the particles are intentionally stopped at the collimators to present a more clear picture. When simulating the power deposition in the elements then they are not killed. 


\section{Twiss functions and beam profile of the Two-Dipole configuration}

In Fig. 67, the $\sigma_{x y}$ size of the positron beam is plotted in the Two-Dipole lattice design. The $\beta_{x y}$ functions are shown in Fig. 68. The $\beta_{x y}$ and dispersion $\left(\mathrm{D}_{x}\right)$ functions are plotted in Fig. 69 for the first few meters of this configuration. The dispersion is introduced by the first dipole, which has a relation with off-momentum particles as given in Eq. (14). In this configuration we use a DBA structure as introduced in Sec. I.1.5. In Fig. 69 , the $\mathrm{D}_{x}$ goes up to $5 \mathrm{~cm}$, where the quadrupole at the symmetry point of the DBA gives a focusing kick in the bending direction, as a result of that the dispersion and its derivatives eventually vanish at the end of the dipoles.

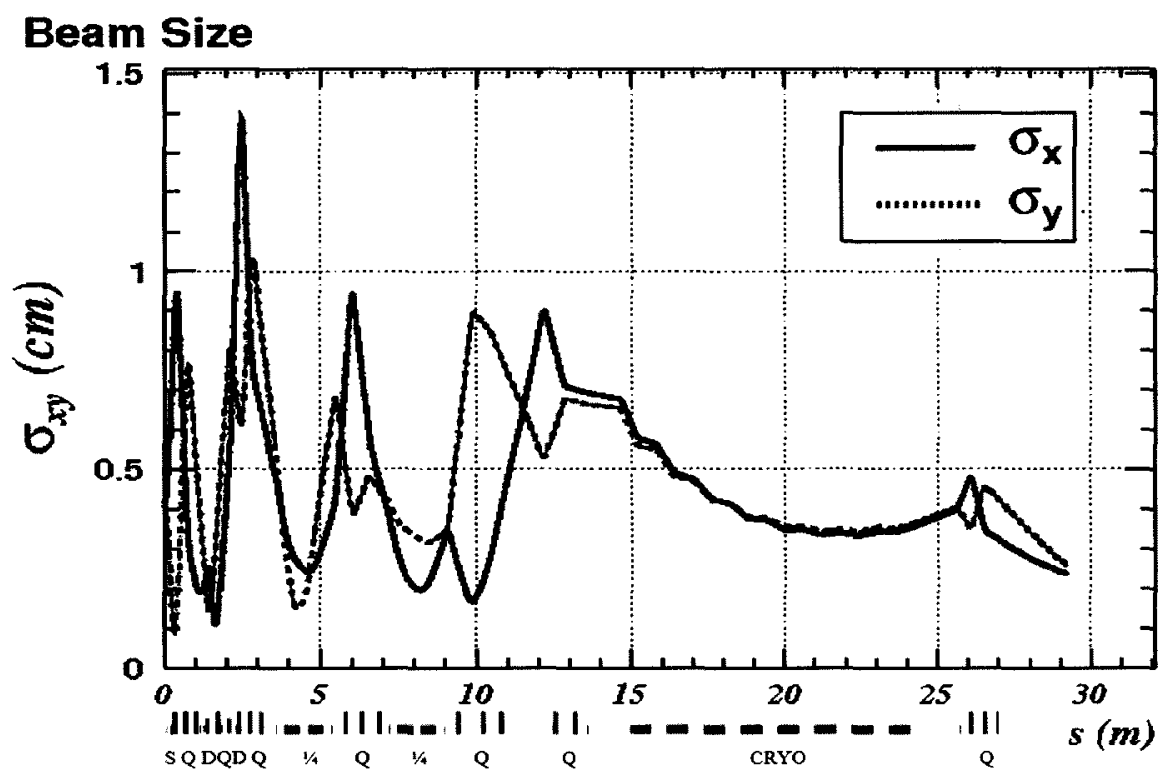

FIG. 67: The 1- $\sigma$ beam size of the positron beam as a function of the central orbit in Two-Dipole configuration. The solid line is $\sigma_{x}$ and dashed line is $\sigma_{y}$. The boxes under the horizontal axis show the location of the magnets, where the solenoid (S), dipoles (D), quadrupoles (Q), quarter cryomodules (1/4) and full cryomodule (CRYO).

In Table 15, the efficiency, equivalent current with a $10 \mathrm{~mA}$ incoming electron beam, the positron momentum, the geometrical transverse emittance $\varepsilon_{x y}$ and time spread of the positrons at different BPM locations are given. This table was prepared by using $\mathrm{G} 4$ tracking results.

The full beam envelopes of the positron beam as plotted in Figs. 70 and 71, give full information about the beam size. These figures are the results of G4BEAMLINE tracking, which all the particles (selected cut positrons) are plotted. The total beam 


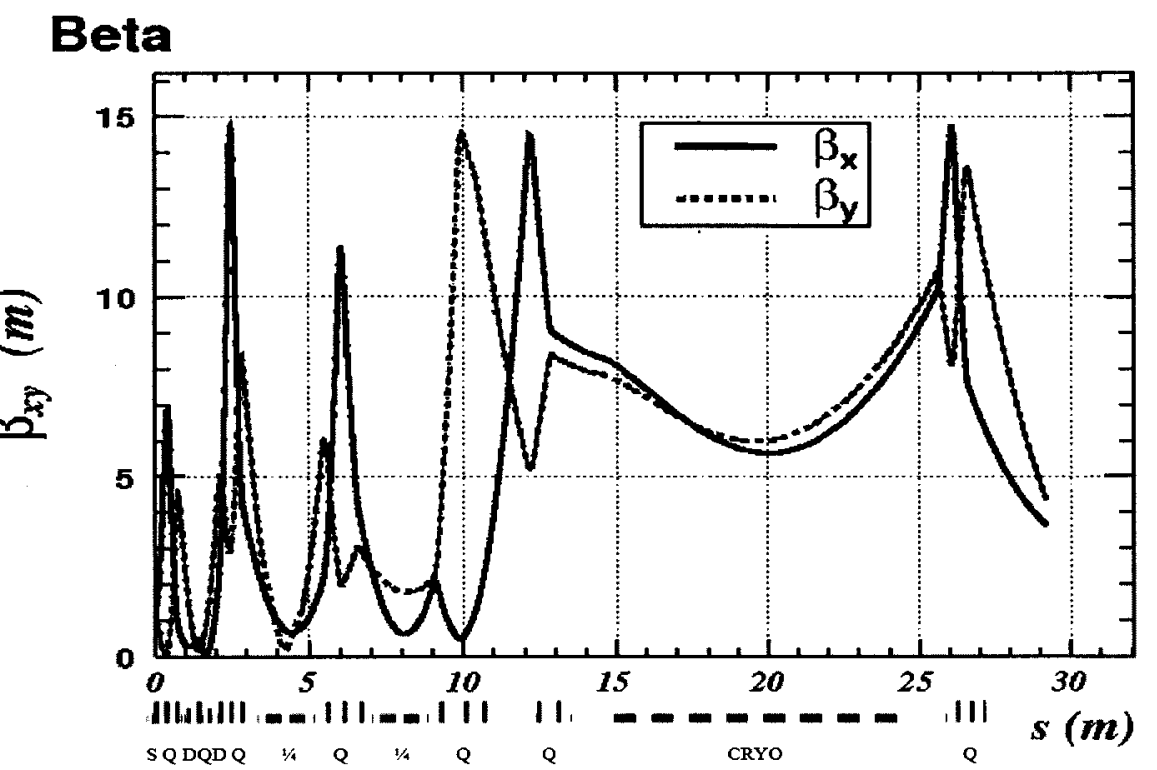

FIG. 68: The twiss $\beta_{x y}$ functions of the positron beam as a function of the central orbit are plotted in Two-Dipole configuration. The solid line is $\beta_{x}$ and dashed line is $\beta_{y}$. The boxes under the horizontal axis show the location of the magnets, where the solenoid (S), dipoles (D), quadrupoles (Q), quarter cryomodules (1/4) and full cryomodule (CRYO).

size goes up to $5 \mathrm{~cm}$ at certain locations, which is larger than the CFM full beam profile. These locations are usually the mid-points of the quadrupoles. As it can be seen from the graphs, the beam sizes are the lowest in size in the cryomodules. Since the full cryomodule is long $(8 \mathrm{~m})$, the beam is brought to a maximum size and focused to a waist position in the middle of the full $\mathrm{C} 100$ cryomodule allowing the positron beam to make till the end of 100 . In this configuration as well, the positrons at large angles are lost when they hit the cryomodules.

At the end of the lattice, the positron distributions have outliers, which were biasing the emittance and time $\&$ momentum values. As a result of that, a recalculation of the r.m.s values was completed by collimating (hard cut) the beam transverse parameters at $2.5 \sigma_{o l d}$. In Fig. 72 , the $p, t, x, y$ and transverse phase space areas at the connection point of North Linac (at BPM \# iIPM10 in this configuration) are plotted with and without outliers. The new r.m.s values give better numbers while we lose a portion of the beam current as provided in Table 15.

At the end of the Two-Dipole configuration, there is $\sim 2.7 \mu \mathrm{A}$ of positron current 
within the admittance values of CEBAF under the assumptions of $10 \mathrm{~mA} 126 \mathrm{MeV}$ electron beam hitting on a $2 \mathrm{~mm}$ tungsten with $\sigma=100 \mu \mathrm{m}$ spot size. The last row of Table 15 was prepared by using the recalculated r.m.s values from the recalculated r.ms. plots.

The emittance values at the end of the lattice are almost same both in the TwoDipole and CFM solutions. In this Two-Dipole lattice, the r.m.s energy spread has stayed the same as opposed to the slight growth as in the CFM lattice. Time spread is slightly larger than the CFM configuration though. This can be explained with the lower total bend angle in the CFM lattice, where off-momentum positrons at large angles are kicked more in the Two-Dipole configuration, causing to take a longer path. The full lattice design parameters such as $s$ positions, length and field values of the magnetic elements as well as the twiss parameters at the end of these elements are provided in Table 16.

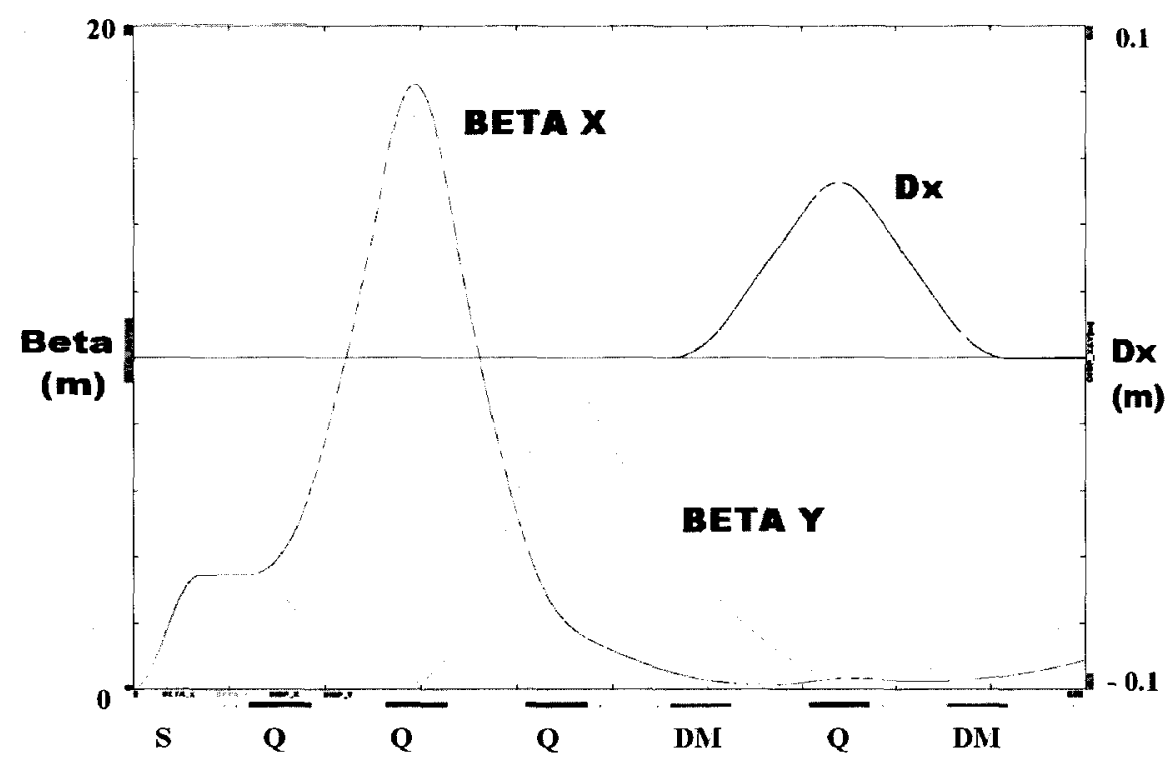

FIG. 69: This plot zooms into the first few meters of the Two-Dipole lattice. Only quadrupoles, dipoles and solenoid are shown at this lattice part. BetaX and BetaY are twiss $\beta$ functions and $\mathbf{D x}$ is the dispersion function. $\mathbf{S}$ at the beginning of the lattice is the Solenoid, Qs are the quadrupoles and DMs are the dipoles. 
TABLE 15: The efficiency and equivalent current at $10 \mathrm{~mA}$ incoming electron beam, momentum, geometrical emittance and time spread information of the positron beam for the two-dipole lattice at different BPMs in the lattice. In this table these values are obtained by propagating the cut positrons from G4 simulation code. The output data to create this table is filtered via a cut to trim the outliers. The outliers were about $10 \%$.

\begin{tabular}{ccccccc}
\hline \hline Detector & $\begin{array}{c}\text { Efficiency } \\
\left(10^{-4} e^{+} / e^{-}\right)\end{array}$ & $\begin{array}{c}\text { Current } \\
(\mu A)\end{array}$ & $\begin{array}{c}p \pm \sigma_{p} \\
(\mathrm{MeV} / \mathrm{c})\end{array}$ & $\begin{array}{c}\varepsilon_{X} \\
(\mathrm{~mm} \cdot \mathrm{mrad})\end{array}$ & $\begin{array}{c}\varepsilon_{Y} \\
(\mathrm{~mm} \cdot \mathrm{mrad})\end{array}$ & $\begin{array}{c}\sigma_{t} \\
(\mathrm{ps})\end{array}$ \\
\hline Source & 3.6 & 3.6 & $15.0 \pm 0.5$ & 5.3 & 5.3 & 0.1 \\
iIPM0 & 3.6 & 3.6 & $15.0 \pm 0.5$ & 13.3 & 13.3 & 0.7 \\
iIPM4 & 3.3 & 3.3 & $25.0 \pm 0.5$ & 12 & 9 & 2.4 \\
iIPM6 & 3.1 & 3.1 & $35.0 \pm 0.5$ & 7 & 6 & 2.7 \\
iIPM10 & 2.7 & 2.7 & $126.0 \pm 0.5$ & 1.6 & 1.8 & 2.1 \\
\hline \hline
\end{tabular}

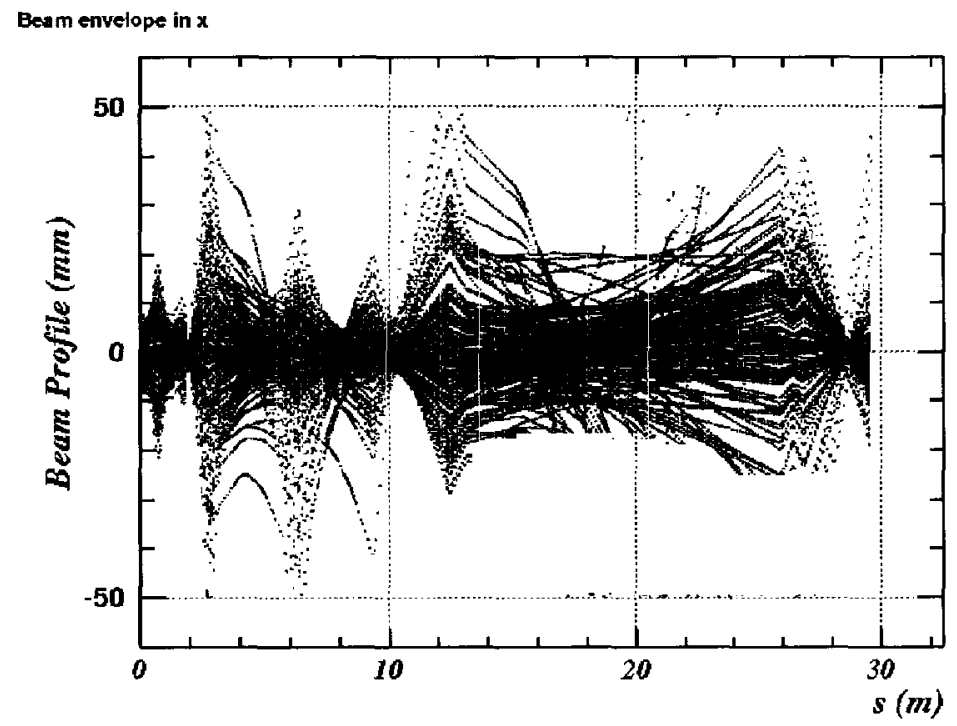

FIG. 70: The full beam envelope of the cut positron beam in $x$. This is the simulation result from the G4 propagated through the Two-Dipole lattice. 


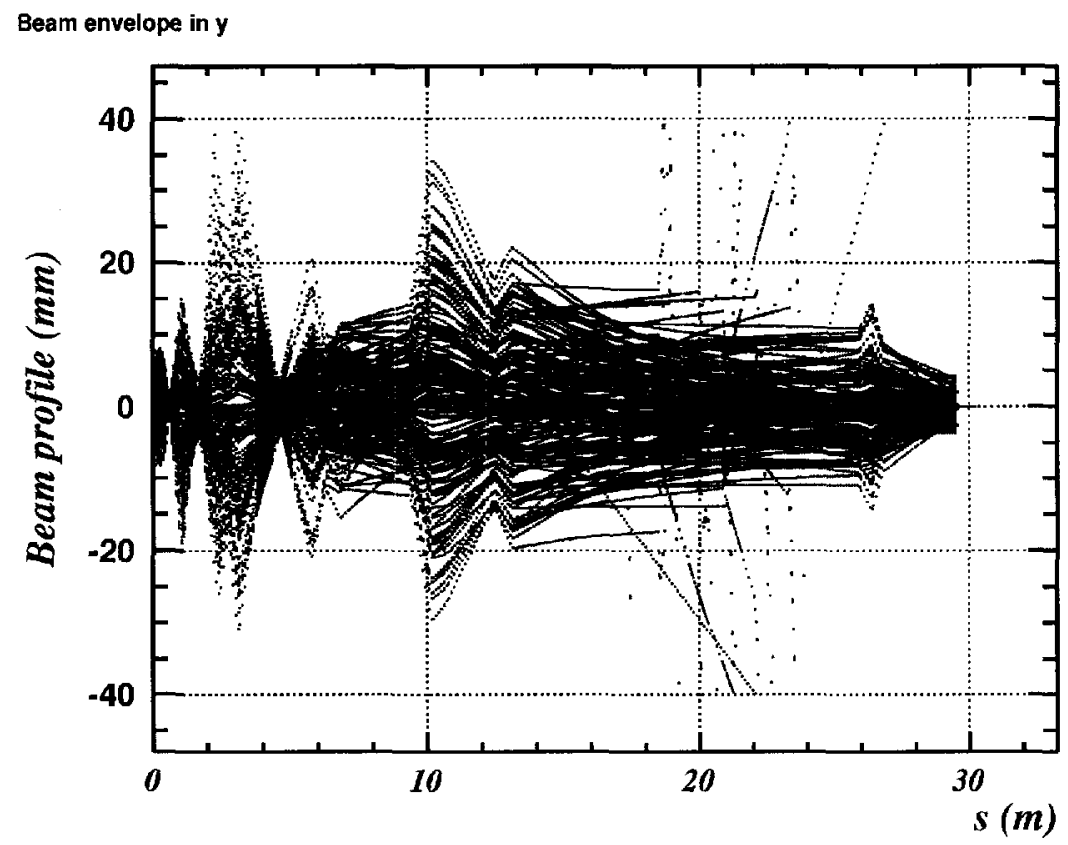

FIG. 71: The full beam envelope of the cut positron beam in $y$. This is the simulation result from the G4 propagated through the Two-Dipole lattice. 


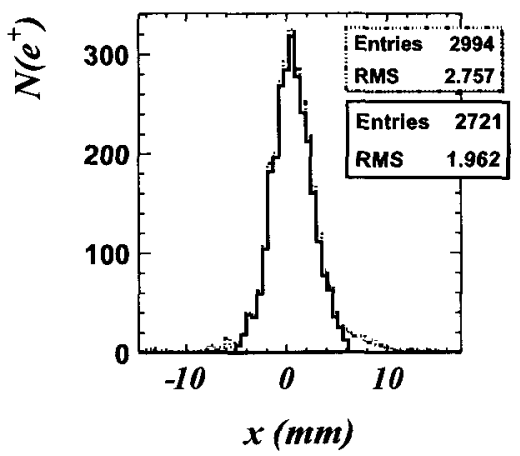

(a)

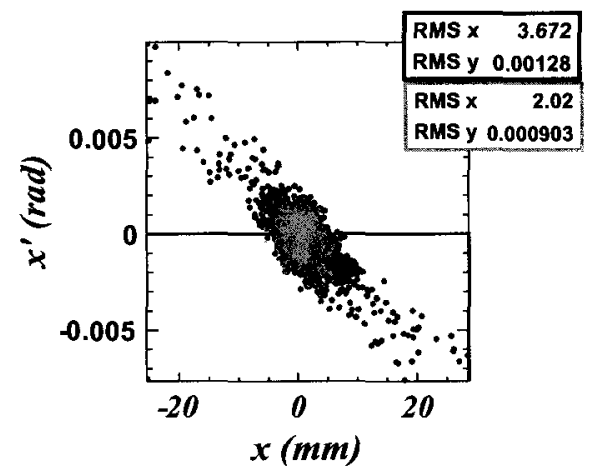

(c)

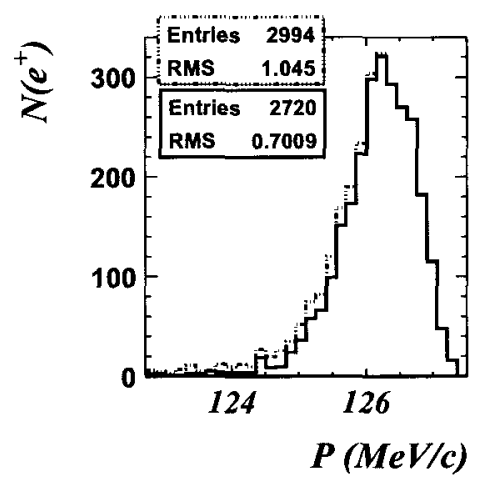

(e)

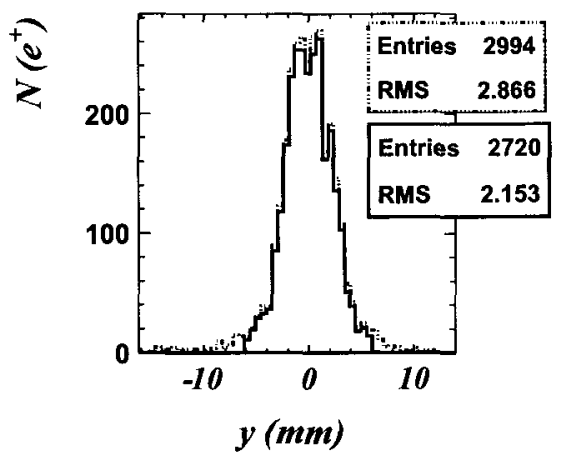

(b)

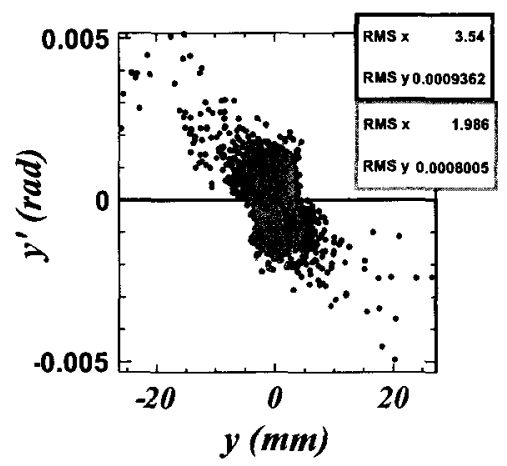

(d)

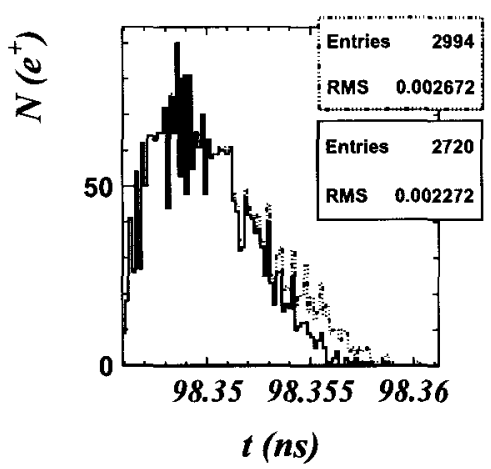

(f)

FIG. 72: The positron beam at the end of the Two-Dipole lattice. In 1D plots, the dashed lines show the selected $\mathrm{e}^{+}$, which reach to the North Linac connection point. These $\mathrm{e}^{+}$have outliers extending far from the mean values. So recalculation of the r.m.s values were necessary. This was completed by putting a collimator (hard cut) at $2.5 \mathrm{r} . \mathrm{m} . \mathrm{s}_{\text {old }}$, and recalculating the r.m.s $\mathrm{s}_{\text {new }}$ values accordingly. The solid lines in the figures show the recalculated r.m.s values. In 2D transverse phase space plots, shown in (c) and (d), the darker area is the selected positrons and the lighter area is the same positrons but without outliers. These plots are tracking results from G4BEAMLINE simulation. 
TABLE 16: Lattice description of the Two-Dipole configuration. The dipole field values of the sector dipoles are: b01 $=0.5822 k G$, b02 $=0.5822 k G$ with each giving a kick in the same direction with a $\theta=10$ degrees for a total of 20 degrees. Solenoid $\mathrm{C} 01$ has a field $\mathrm{B}=9.8 \mathrm{kG}$. Initial geometrical emittances given as input are $\varepsilon_{x y}=5.8$ $\operatorname{mm} . \operatorname{mrad}$ at $p\left(e^{+}\right)=15 \mathrm{MeV} / \mathrm{c}$.

\begin{tabular}{|c|c|c|c|c|c|c|c|c|c|}
\hline \hline $\mathbf{N}$ & Name & Type & $\begin{array}{c}\mathbf{S} \\
{[\mathbf{c m}]}\end{array}$ & $\begin{array}{c}\mathbf{L} \\
{[\mathbf{c m}]}\end{array}$ & $\begin{array}{c}\mathbf{G} \\
{[\mathbf{k G} / \mathbf{c m}]}\end{array}$ & $\begin{array}{c}\beta_{x} \\
{[\mathbf{c m}]}\end{array}$ & $\begin{array}{c}\alpha_{x} \\
-\end{array}$ & $\begin{array}{c}\beta_{y} \\
{[\mathbf{c m}]}\end{array}$ & $\begin{array}{c}\alpha_{y} \\
-\end{array}$ \\
\hline \hline 0 & START & & 0 & - & & 0.3 & -0.1 & 0.3 & -0.1 \\
\hline 1 & C01 & Solenoid & 16 & 16 & & 344 & -0.1 & 346 & -0.1 \\
\hline 2 & oDAB0 & Drift & 22 & 6 & & 344 & -0.1 & 347 & -0.1 \\
\hline 3 & HC & Collim & 22 & 0 & & 344 & -0.1 & 347 & -0.1 \\
\hline 4 & oD00 & Drift & 25 & 3 & & 345 & -0.1 & 348 & -0.1 \\
\hline 5 & iIPM0 & BPM & 25 & 0 & & 345 & -0.1 & 348 & -0.1 \\
\hline 6 & oD00 & Drift & 28 & 3 & & 345 & -0.1 & 348 & -0.1 \\
\hline 7 & qA1 & Quad & 43 & 15 & -0.135 & 231 & -7.8 & 67 & 3.4 \\
\hline 8 & oDA1 & Drift & 61 & 18 & & 596 & -12.6 & 5 & 0.1 \\
\hline 9 & qA2 & Quad & 76 & 15 & 0.1432 & 572 & 13.8 & 58 & -4.3 \\
\hline 10 & oDA2 & Drift & 95 & 19 & & 167 & 7.4 & 343 & -10.7 \\
\hline 11 & qA3 & Quad & 110 & 15 & -0.1426 & 61 & 1.1 & 428 & 6.3 \\
\hline 12 & oD00 & Drift & 113 & 3 & & 55 & 1.0 & 391 & 6.0 \\
\hline 13 & iIPM1 & BPM & 113 & 0 & & 55 & 1.0 & 391 & 6.0 \\
\hline 14 & oDA3 & Drift & 127 & 14 & & 35 & 0.5 & 241 & 4.7 \\
\hline 15 & HC & Collim & 127 & 0 & & 35 & 0.5 & 241 & 4.7 \\
\hline 16 & oD00 & Drift & 130 & 3 & & 32 & 0.4 & 213 & 4.4 \\
\hline 17 & b01 & Dipole & 145 & 15 & & 28 & -0.1 & 103 & 3.0 \\
\hline 18 & oDL0 & Drift & 164 & 19 & & 45 & -0.8 & 24 & 1.2 \\
\hline 19 & qM1 & Quad & 179 & 15 & 0.2302 & 29 & 1.5 & 26 & -1.3 \\
\hline 20 & oD00 & Drift & 182 & 3 & & 21 & 1.1 & 34 & -1.6 \\
\hline 21 & HC & Collim & 182 & 0 & & 21 & 1.1 & 34 & -1.6 \\
\hline & & & Continued on Next Page. & & & & \\
\hline
\end{tabular}


TABLE 16 - Continued

\begin{tabular}{|c|c|c|c|c|c|c|c|c|c|}
\hline $\mathbf{N}$ & Name & Type & $\begin{array}{c}\mathrm{S} \\
{[\mathrm{cm}]}\end{array}$ & $\begin{array}{c}\mathrm{L} \\
{[\mathrm{cm}]}\end{array}$ & $\begin{array}{c}\mathrm{G} \\
{[\mathrm{kG} / \mathrm{cm}]}\end{array}$ & $\begin{array}{c}\beta_{x} \\
{[\mathrm{~cm}]}\end{array}$ & $\begin{array}{r}\alpha_{x} \\
-\end{array}$ & $\begin{array}{c}\beta_{y} \\
{[\mathbf{c m}]}\end{array}$ & $\begin{array}{c}\alpha_{y} \\
-\end{array}$ \\
\hline 22 & oDL1 & Drift & 198 & 16 & & 13 & -0.6 & 110 & -3.2 \\
\hline 23 & b02 & Dipole & 213 & 15 & & 56 & -2.2 & 229 & -4.7 \\
\hline 24 & oDB0 & Drift & 229 & 16 & & 154 & -3.9 & 405 & -6.3 \\
\hline 25 & $\mathrm{HC}$ & Collim & 229 & 0 & & 154 & -3.9 & 405 & -6.3 \\
\hline 26 & oD00 & Drift & 232 & 3 & & 179 & -4.2 & 444 & -6.6 \\
\hline 27 & iIPM2 & BPM & 232 & 0 & & 179 & -4.2 & 444 & -6.6 \\
\hline 28 & qB1 & Quad & 247 & 15 & -0.08 & 438 & -15.0 & 478 & 4.7 \\
\hline 29 & oDB1 & Drift & 267 & 20 & & 1247 & -25.4 & 310 & 3.7 \\
\hline 30 & $\mathrm{qB} 2$ & Quad & 282 & 15 & 0.115 & 1332 & 20.8 & 359 & -7.5 \\
\hline 31 & oDB2 & Drift & 302 & 20 & & 630 & 14.3 & 721 & -10.6 \\
\hline 32 & $\mathrm{qB} 3$ & Quad & 317 & 15 & -0.07 & 406 & 2.1 & 806 & 5.6 \\
\hline 33 & oDB3 & Drift & 357 & 40 & & 256 & 1.6 & 423 & 4.0 \\
\hline 34 & iIPM3 & BPM & 357 & 0 & & 256 & 1.6 & 423 & 4.0 \\
\hline 35 & oD1006 & Drift & 382 & 25 & & 185 & 1.2 & 249 & 3.0 \\
\hline 36 & aC50_1 & $\mathrm{RF}$ & 432 & 50 & & 91 & 0.6 & 47 & 1.0 \\
\hline 37 & oD1006 & Drift & 457 & 25 & & 70 & 0.2 & 24 & -0.1 \\
\hline 38 & aC50_1 & $\mathrm{RF}$ & 507 & 50 & & 83 & -0.5 & 135 & -2.1 \\
\hline 39 & oD1006 & Drift & 532 & 25 & & 117 & -0.9 & 268 & -3.2 \\
\hline 40 & iIPM4 & $\mathrm{BPM}$ & 532 & 0 & & 117 & -0.9 & 268 & -3.2 \\
\hline 41 & oDC0 & Drift & 570 & 38 & & 205 & -1.4 & 571 & -4.8 \\
\hline 42 & $\mathrm{qC} 1$ & Quad & 585 & 15 & -0.093 & 318 & -6.8 & 561 & 5.4 \\
\hline 43 & oDC1 & Drift & 622 & 37 & & 1024 & -12.3 & 236 & 3.4 \\
\hline 44 & $\mathrm{qC} 2$ & Quad & 637 & 15 & 0.109 & 1082 & 8.8 & 202 & -0.9 \\
\hline 45 & oDC2 & Drift & 676 & 39 & & 507 & 6.0 & 289 & -1.3 \\
\hline 46 & qC3 & Quad & 691 & 15 & -0.04 & 388 & 2.2 & 297 & 0.8 \\
\hline 47 & oDC3 & Drift & 716 & 25 & & 287 & 1.8 & 262 & 0.6 \\
\hline 48 & iIPM5 & BPM & 716 & 0 & & 287 & 1.8 & 262 & 0.6 \\
\hline 49 & oD1006 & Drift & 741 & 25 & & 204 & 1.5 & 234 & 0.5 \\
\hline 50 & $\mathrm{aC} 50 \_2$ & $\mathrm{RF}$ & 791 & 50 & & 95 & 0.7 & 194 & 0.3 \\
\hline \multicolumn{10}{|c|}{ Continued on Next Page... } \\
\hline
\end{tabular}


TABLE 16 - Continued

\begin{tabular}{|c|c|c|c|c|c|c|c|c|c|}
\hline $\mathbf{N}$ & Name & Type & $\begin{array}{c}\mathrm{S} \\
{[\mathrm{cm}]}\end{array}$ & $\begin{array}{c}\mathbf{L} \\
{[\mathrm{cm}]}\end{array}$ & $\begin{array}{c}\mathrm{G} \\
{[\mathrm{kG} / \mathrm{cm}]}\end{array}$ & $\begin{array}{c}\beta_{x} \\
{[\mathrm{~cm}]}\end{array}$ & $\alpha_{x}$ & $\begin{array}{c}\beta_{y} \\
{[\mathrm{~cm}]}\end{array}$ & $\begin{array}{c}\alpha_{y} \\
- \\
\end{array}$ \\
\hline 51 & oD1006 & Drift & 816 & 25 & & 69 & 0.3 & 183 & 0.1 \\
\hline 52 & aC50_2 & $\mathrm{RF}$ & 866 & 50 & & 77 & -0.5 & 181 & -0.1 \\
\hline 53 & oD1006 & Drift & 891 & 25 & & 110 & -0.9 & 190 & -0.2 \\
\hline 54 & iIPM6 & BPM & 891 & 0 & & 110 & -0.9 & 190 & -0.2 \\
\hline 55 & oDC0 & Drift & 929 & 38 & & 199 & -1.5 & 216 & -0.5 \\
\hline 56 & $\mathrm{qD} 1$ & Quad & 944 & 15 & 0.1353 & 193 & 1.8 & 293 & -5.1 \\
\hline 57 & oDD1 & Drift & 1008 & 64 & & 53 & 0.4 & 1311 & -10.9 \\
\hline 58 & $\mathrm{qD} 2$ & Quad & 1023 & 15 & -0.075 & 52 & -0.4 & 1459 & 1.4 \\
\hline 59 & oDD2 & Drift & 1066 & 43 & & 124 & -1.3 & 1339 & 1.4 \\
\hline 60 & qD3 & Quad & 1081 & 15 & -0.01 & 171 & -1.8 & 1274 & 3.0 \\
\hline 61 & oDD3 & Drift & 1236 & 155 & & 1364 & -5.8 & 539 & 1.8 \\
\hline 62 & iIPM7 & $\mathrm{BPM}$ & 1236 & 0 & & 1364 & -5.8 & 539 & 1.8 \\
\hline 63 & oD00 & Drift & 1239 & 3 & & 1399 & -5.9 & 529 & 1.8 \\
\hline 64 & $\mathrm{qE} 1$ & Quad & 1254 & 15 & 0.0701 & 1413 & 5.0 & 537 & -2.4 \\
\hline 65 & oDE1 & Drift & 1303 & 49 & & 966 & 4.1 & 797 & -2.9 \\
\hline 66 & $\mathrm{qE} 2$ & Quad & 1318 & 15 & -0.031 & 901 & 0.3 & 838 & 0.3 \\
\hline 67 & oDE2 & Drift & 1348 & 30 & & 883 & 0.3 & 823 & 0.2 \\
\hline 68 & iIPM8 & BPM & 1348 & 0 & & 883 & 0.3 & 823 & 0.2 \\
\hline 69 & oD1005 & Drift & 1459 & 111 & & 835 & 0.1 & 788 & 0.1 \\
\hline 70 & oD1006 & Drift & 1484 & 25 & & 829 & 0.1 & 784 & 0.1 \\
\hline 71 & aC100 & $\mathrm{RF}$ & 1554 & 70 & & 792 & 0.4 & 758 & 0.3 \\
\hline 72 & oD1006 & Drift & 1579 & 25 & & 775 & 0.3 & 744 & 0.3 \\
\hline 73 & oD1006 & Drift & 1604 & 25 & & 759 & 0.3 & 732 & 0.2 \\
\hline 74 & aC100 & RF & 1674 & 70 & & 711 & 0.4 & 695 & 0.3 \\
\hline 75 & oD1006 & Drift & 1699 & 25 & & 693 & 0.3 & 682 & 0.3 \\
\hline 76 & oD1006 & Drift & 1724 & 25 & & 677 & 0.3 & 670 & 0.2 \\
\hline 77 & $\mathrm{aC} 100$ & $\mathrm{RF}$ & 1794 & 70 & & 635 & 0.3 & 639 & 0.2 \\
\hline 78 & oD1006 & Drift & 1819 & 25 & & 621 & 0.3 & 629 & 0.2 \\
\hline 79 & oD1006 & Drift & 1844 & 25 & & 609 & 0.2 & 621 & 0.1 \\
\hline \multicolumn{10}{|c|}{ Continued on Next Page. } \\
\hline
\end{tabular}


TABLE 16 - Continued

\begin{tabular}{|c|c|c|c|c|c|c|c|c|c|}
\hline \hline $\mathbf{N}$ & Name & Type & $\begin{array}{c}\mathbf{S} \\
{[\mathbf{c m}]}\end{array}$ & $\begin{array}{c}\mathbf{L} \\
{[\mathbf{c m}]}\end{array}$ & $\begin{array}{c}\mathbf{G} \\
{[\mathbf{k G} / \mathbf{c m}]}\end{array}$ & $\begin{array}{c}\beta_{x} \\
{[\mathbf{c m}]}\end{array}$ & $\begin{array}{c}\alpha_{x} \\
-\end{array}$ & $\begin{array}{c}\beta_{y} \\
{[\mathbf{c m}]}\end{array}$ & $\begin{array}{c}\alpha_{y} \\
-\end{array}$ \\
\hline \hline 80 & aC100 & RF & 1914 & 70 & & 583 & 0.2 & 604 & 0.1 \\
\hline 81 & oD1006 & Drift & 1939 & 25 & & 576 & 0.1 & 601 & 0.1 \\
\hline 82 & oD1006 & Drift & 1964 & 25 & & 571 & 0.1 & 599 & 0.0 \\
\hline 83 & aC100 & RF & 2034 & 70 & & 566 & 0.0 & 602 & 0.0 \\
\hline 84 & oD1006 & Drift & 2059 & 25 & & 567 & 0.0 & 605 & -0.1 \\
\hline 85 & oD1006 & Drift & 2084 & 25 & & 570 & -0.1 & 611 & -0.1 \\
\hline 86 & aC100 & RF & 2154 & 70 & & 588 & -0.2 & 634 & -0.2 \\
\hline 87 & oD1006 & Drift & 2179 & 25 & & 597 & -0.2 & 645 & -0.2 \\
\hline 88 & oD1006 & Drift & 2204 & 25 & & 609 & -0.3 & 659 & -0.3 \\
\hline 89 & aC100 & RF & 2274 & 70 & & 651 & -0.3 & 704 & -0.4 \\
\hline 90 & oD1006 & Drift & 2299 & 25 & & 669 & -0.4 & 723 & -0.4 \\
\hline 91 & oD1006 & Drift & 2324 & 25 & & 689 & -0.4 & 744 & -0.4 \\
\hline 92 & aC100 & RF & 2394 & 70 & & 754 & -0.5 & 810 & -0.5 \\
\hline 93 & oD1006 & Drift & 2419 & 25 & & 781 & -0.6 & 837 & -0.6 \\
\hline 94 & oD1008 & Drift & 2555 & 136 & & 961 & -0.8 & 1016 & -0.8 \\
\hline 95 & iIPM9 & BPM & 2555 & 0 & & 961 & -0.8 & 1016 & -0.8 \\
\hline 96 & oDF0 & Drift & 2585 & 30 & & 1010 & -0.8 & 1063 & -0.8 \\
\hline 97 & qF1 & Quad & 2600 & 15 & -0.115 & 1099 & -5.3 & 1023 & 3.5 \\
\hline 98 & oDF1 & Drift & 2630 & 30 & & 1439 & -6.1 & 827 & 3.1 \\
\hline 99 & qF2 & Quad & 2645 & 15 & 0.305 & 1385 & 9.5 & 870 & -6.1 \\
\hline 100 & oDF2 & Drift & 2675 & 30 & & 876 & 7.5 & 1276 & -7.4 \\
\hline 101 & qF3 & Quad & 2690 & 15 & -0.21 & 750 & 1.1 & 1353 & 2.4 \\
\hline 102 & oDF3 & Drift & 2729 & 39 & & 666 & 1.0 & 1170 & 2.2 \\
\hline 103 & oD99 & Drift & 2838 & 109 & & 480 & 0.7 & 743 & 1.7 \\
\hline 104 & oD99 & Drift & 2947 & 109 & & 367 & 0.4 & 438 & 1.1 \\
\hline 105 & iIPM10 & BPM & 2947 & 0 & & 367 & 0.4 & 438 & 1.1 \\
\hline 106 & END & & 2946 & & & 367 & 0.4 & 438 & 1.1 \\
\hline & & & & End of Table & & & & \\
\hline \hline
\end{tabular}


The phase space distributions at the end of beam position monitors

The following plots from Fig. 73 to 79 are the simulation tracking results of G4BEAMLINE. After each quadrupole triplet, the dipole set, quarter RF and full RF the $x-x^{\prime}, y-y^{\prime}, p-t$ and $x-y$ distributions of the positron beams are plotted. These positrons are cut positrons as defined earlier in Sec. IV.1. They are tracked until the end of the lattice.
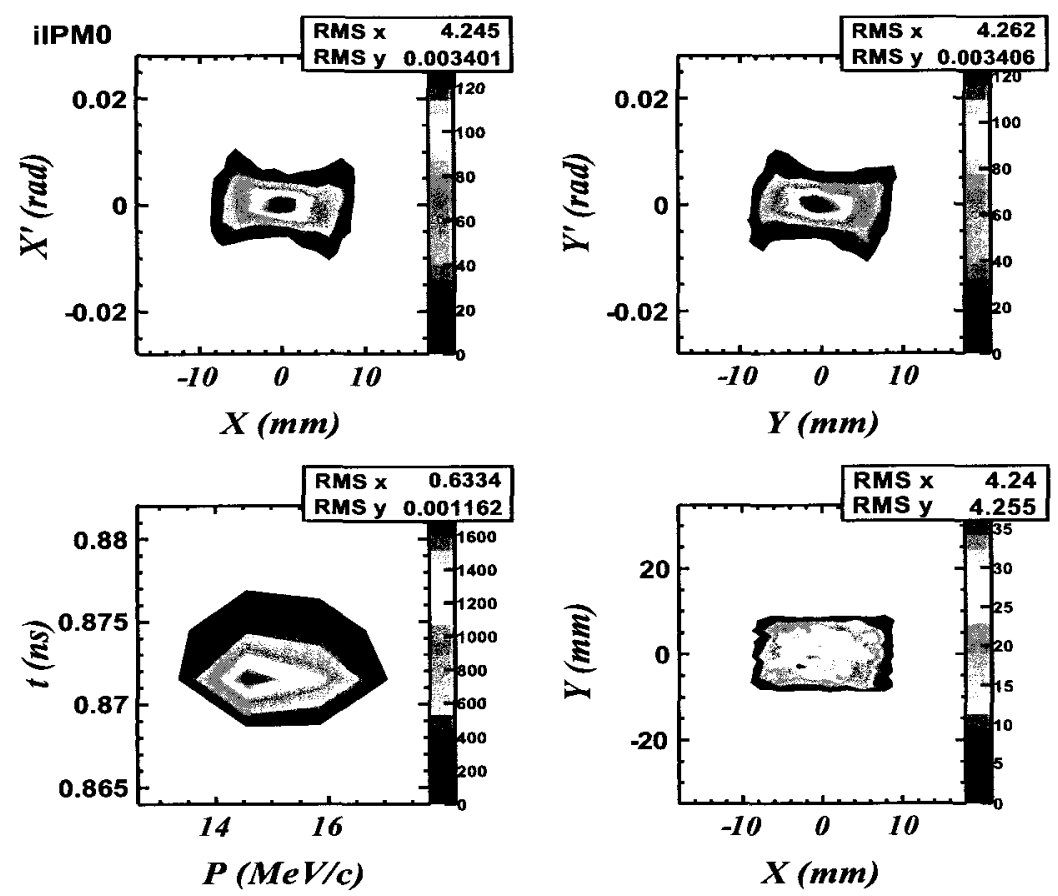

FIG. 73: Transverse and longitudinal phase space of the beam after the solenoid in Two-Dipole lattice. $\varepsilon_{x}=13.3 \mathrm{~mm} \cdot \mathrm{mrad} \quad \varepsilon_{y}=13.3 \mathrm{~mm} \cdot \mathrm{mrad}$. 

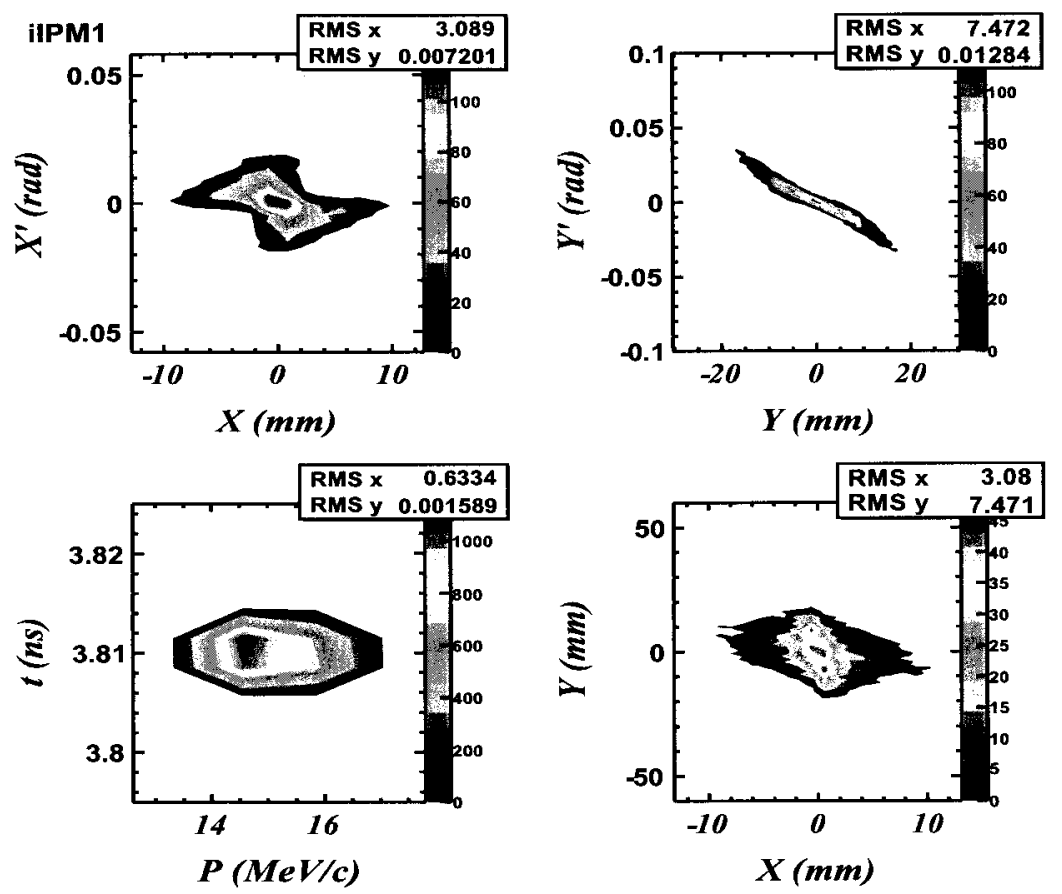

FIG. 74: The beam right before the 1st dipole in Two-Dipole lattice. $\varepsilon_{x}=19$ $\mathrm{mm} \cdot \mathrm{mrad} \varepsilon_{y}=20 \mathrm{~mm} \cdot \mathrm{mrad}$. 

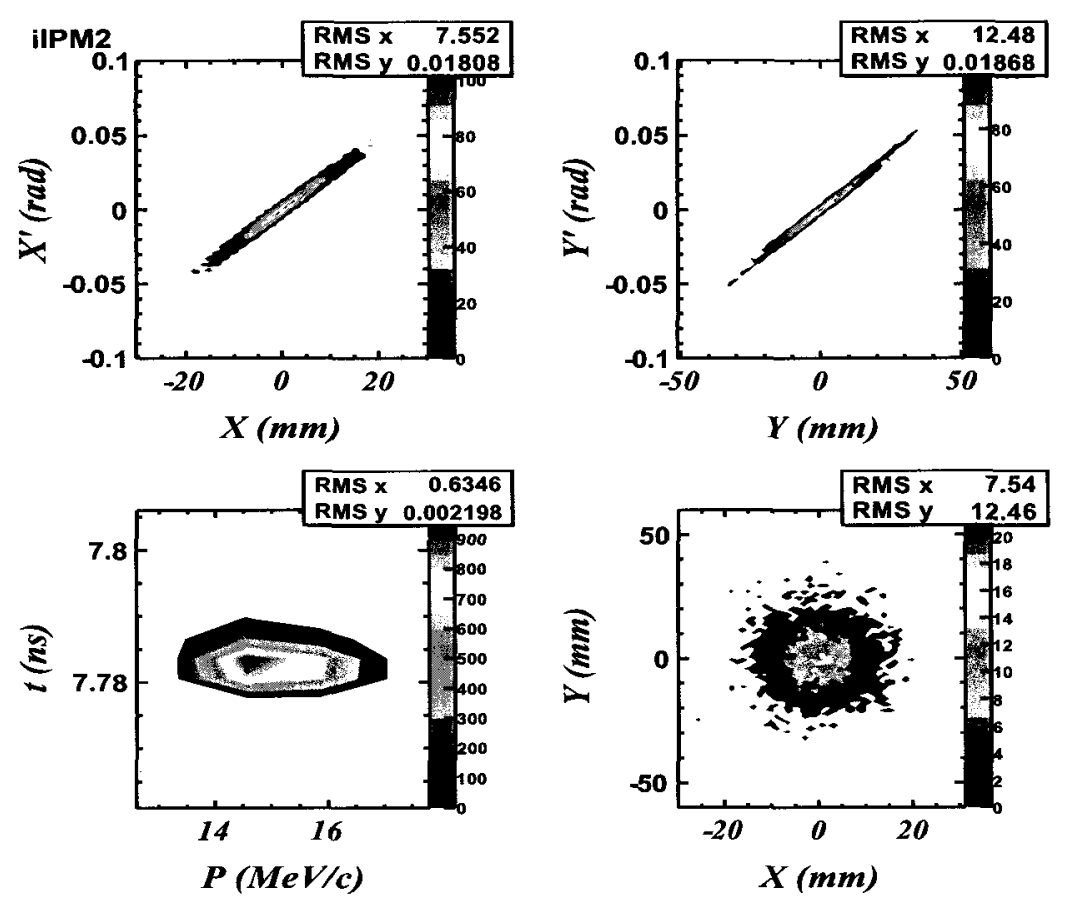

(a)
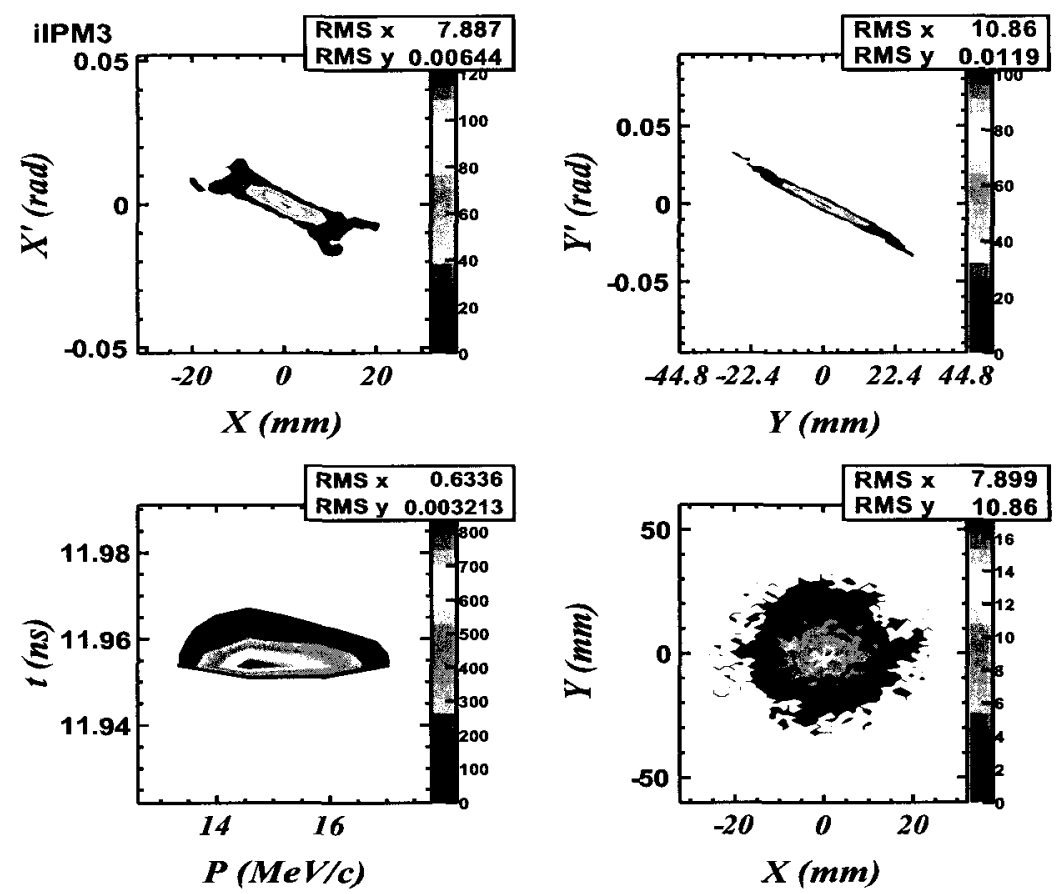

(b)

FIG. 75: (a) Transverse and longitudinal phase space of the beam right after the 2nd dipole in Two-Dipole lattice $\varepsilon_{x}=16 \mathrm{~mm} \cdot \mathrm{mrad} \quad \varepsilon_{y}=18 \mathrm{~mm} \cdot \mathrm{mrad}$. (b) Right before the 1 st quarter cryomodule in $\varepsilon_{x}=18 \mathrm{~mm} \cdot \mathrm{mrad} \quad \varepsilon_{y}=15 \mathrm{~mm} \cdot \mathrm{mrad}$. 

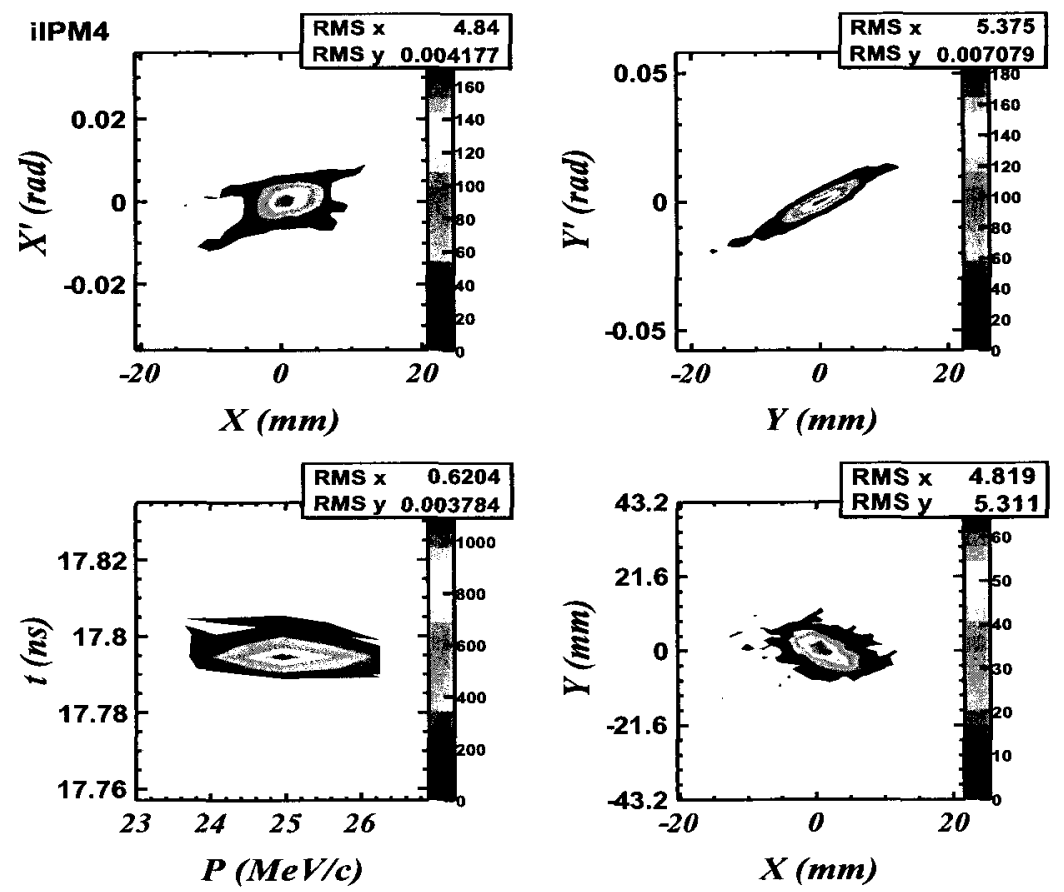

(a)
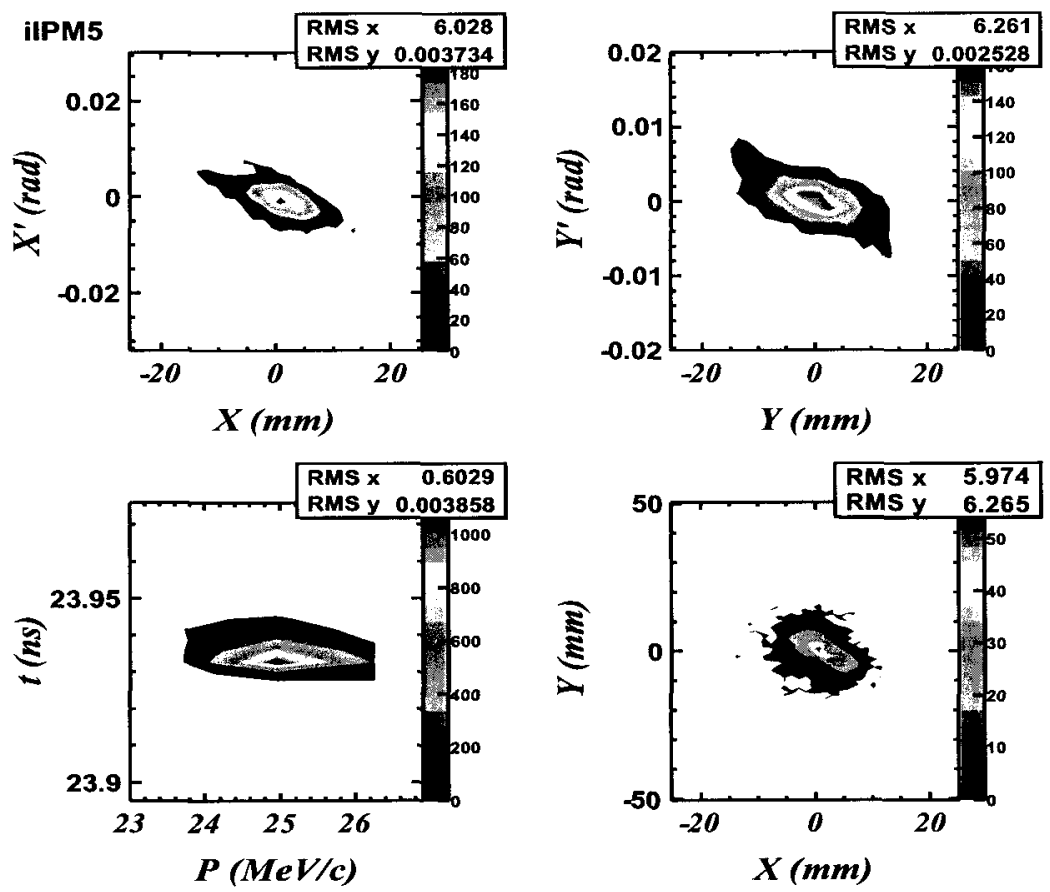

(b)

FIG. 76: (a) Transverse and longitudinal phase space of the beam right after the 1st quarter cryomodule in Two-Dipole lattice $\varepsilon_{x}=12 \mathrm{~mm} \cdot \mathrm{mrad} \quad \varepsilon_{y}=9 \mathrm{~mm} \cdot \mathrm{mrad}(\mathrm{b})$ Right before the 2 nd quarter cryomodule $\varepsilon_{x}=10 \mathrm{~mm} \cdot \mathrm{mrad} \varepsilon_{y}=8 \mathrm{~mm} \cdot \mathrm{mrad}$. 

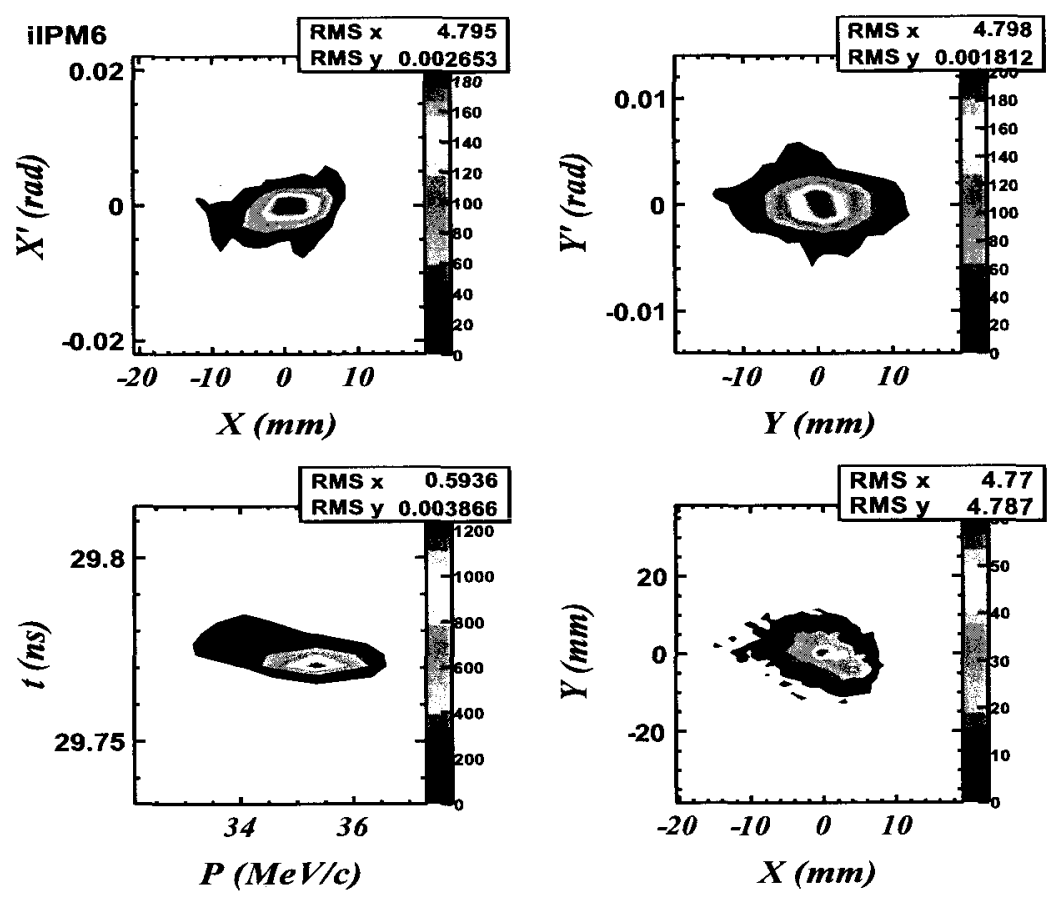

(a)
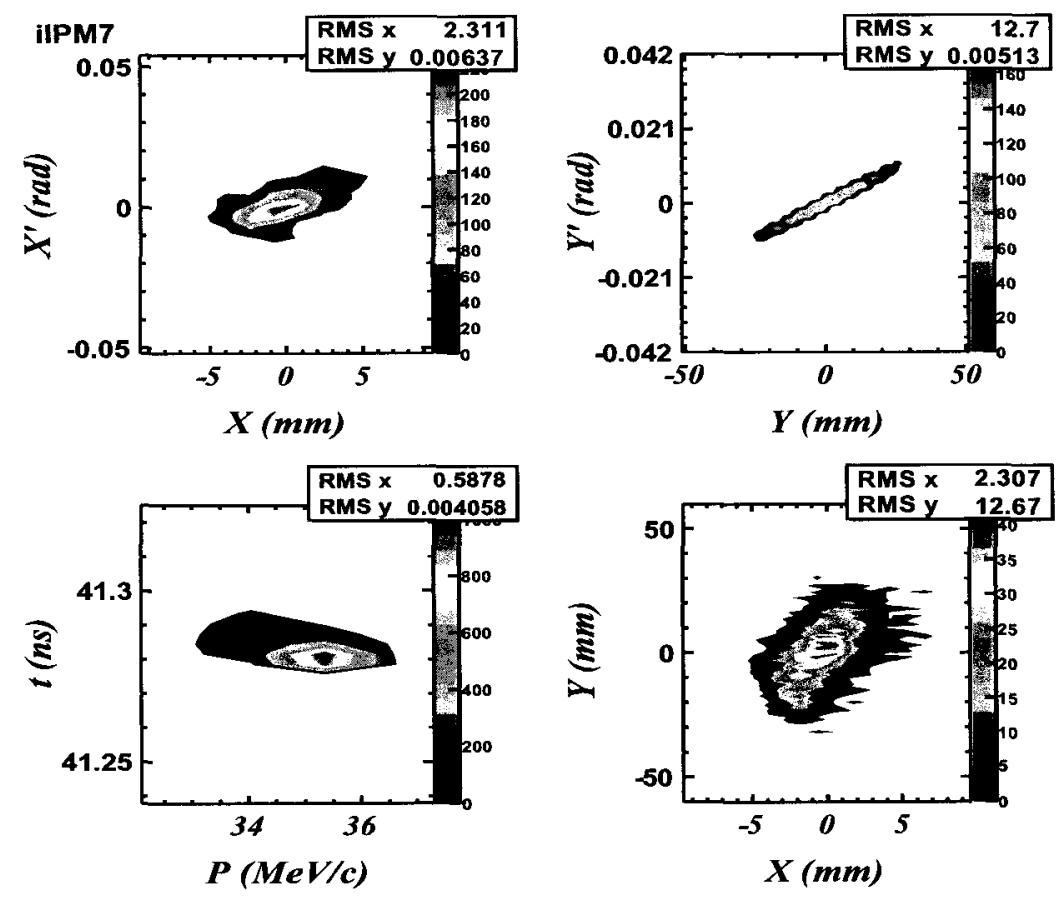

(b)

FIG. 77: (a) Transverse and longitudinal phase space of the beam right after the 2nd quarter cryomodule in Two-Dipole lattice $\varepsilon_{x}=7 \mathrm{~mm} \cdot \mathrm{mrad} \quad \varepsilon_{y}=6 \mathrm{~mm} \cdot \mathrm{mrad}(\mathrm{b})$ Right after the $\mathrm{qD}$ set of quad triplets $\varepsilon_{x}=7 \mathrm{~mm} \cdot \mathrm{mrad} \quad \varepsilon_{y}=6 \mathrm{~mm} \cdot \mathrm{mrad}$. 

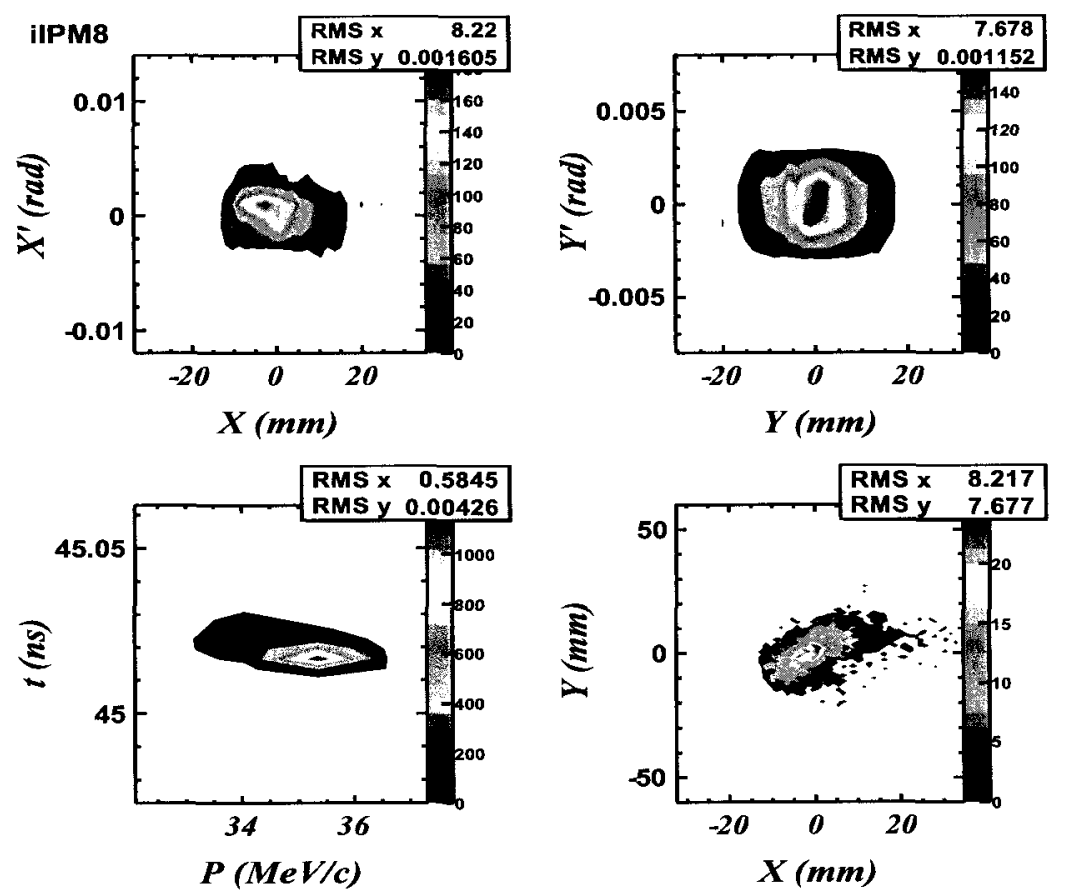

(a)
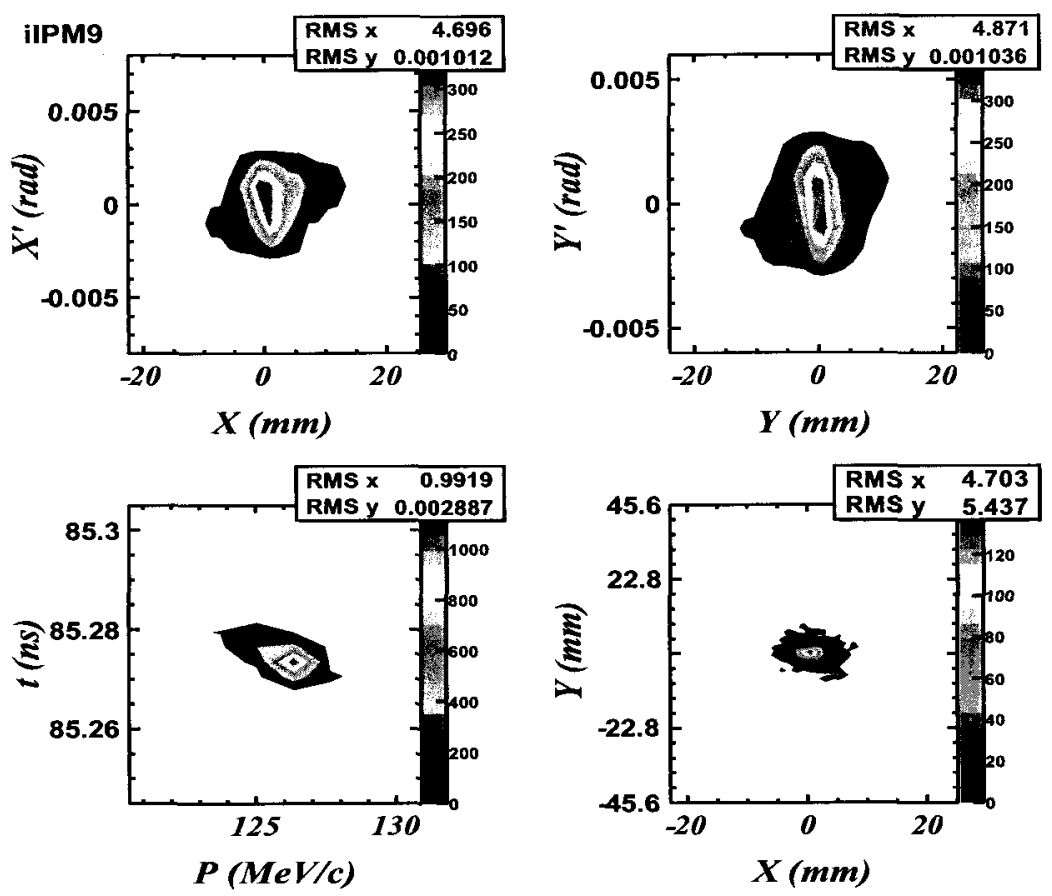

(b)

FIG. 78: (a) Transverse and longitudinal phase space of the beam right before the full cryomodule in Two-Dipole lattice $\varepsilon_{x}=7 \mathrm{~mm} \cdot \mathrm{mrad} \quad \varepsilon_{y}=6 \mathrm{~mm} \cdot \mathrm{mrad}$ (b) Right after the full cryomodule $\varepsilon_{x}=11 \mathrm{~mm} \cdot \mathrm{mrad} \quad \varepsilon_{y}=1.4 \mathrm{~mm} \cdot \mathrm{mrad}$. 

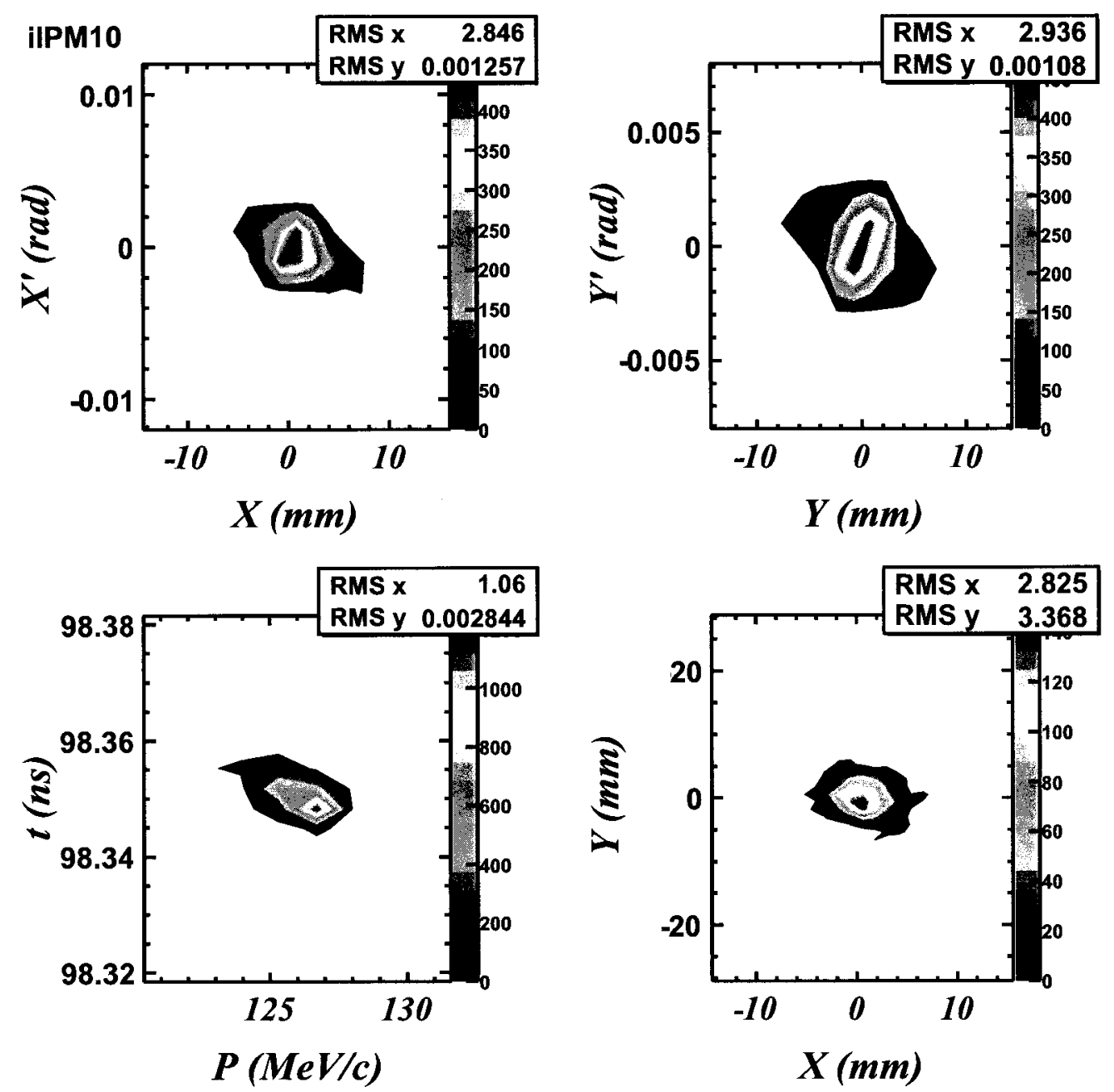

FIG. 79: Transverse and longitudinal phase space of the beam at the end of the lattice where a connection can be made to the North Linac $\varepsilon_{x}=1.6 \mathrm{~mm} \cdot \mathrm{mrad}$ $\varepsilon_{y}=1.8 \mathrm{~mm} \cdot \mathrm{mrad}$. 


\section{IV.5 DESIGN - 3 : MICROTRON DIPOLES}

In this design option, to separate positrons from other secondary particles we propose to use a microtron dipole set instead of using a sector dipole set which is used in 2nd method. A microtron style back-to-back two dipoles are placed together to form this configuration. The positrons are collected by a QWT solenoid followed by a quadrupole triplet. Immediately after this triplet the microtron dipole set is positioned. The biggest advantage of the microtron dipole set is that they do not need quads in between to preserve the achromatic structure making it a compact set of dipoles; the dispersion function is very small (less than $2 \mathrm{~cm}$ ); it is almost isochronous and it has very large energy acceptance. This microtron dipole set should be considered if the large energy spread can be tolerated by the experimental Halls. This microtron dipole system gives an almost unlimited energy acceptance with achromatic and isochronous design. This microtron dipole design follows the lattice structure given in Ref. [53].

A simple schematic drawing is shown in Fig. 80(a), illustrating how the microtron dipoles work. In the figure, two normal dipoles are shown and rotated around y axis ( $\mathrm{x}$ is the bending axis) by half of the bending angle. The beam enters from the long side as opposed to the short side with a glazing angle. Each off-momentum particle follows a different trajectory from the central orbit causing deviation both in $x$ and $x^{\prime}$ if $x$ is taken to be the bending plane. No change occurs in the non-dispersive $y$ plane as it acts as if it was in a drift space. Right after the beam leaves the first microtron dipole, dispersion function becomes linear, and derivative of the dispersion $\eta^{\prime}$ is equal to zero, meaning that different energies travel parallel to each other. But this time $\beta_{y}(s)$ growth occurs which must be taken under control. Fig. 80(b) shows the dispersion function in this configuration.

As this is almost the same configuration with the 2nd method, in this section only twiss $\beta$ functions and the table of the lattice parameters are provided. The twiss $\beta$ s and $1-\sigma$ beam size of the positrons are shown in Fig. 81. The lattice design parameters and corresponding twiss values at the end of these elements are given in Table 17. In the microtron dipoles, the magnetic field used is $0.58218 \mathrm{kG}$, which each dipole gives a 10 degree kick to the beam resulting a total deviation from the 0 -degreee line $\theta=20$ degrees. 


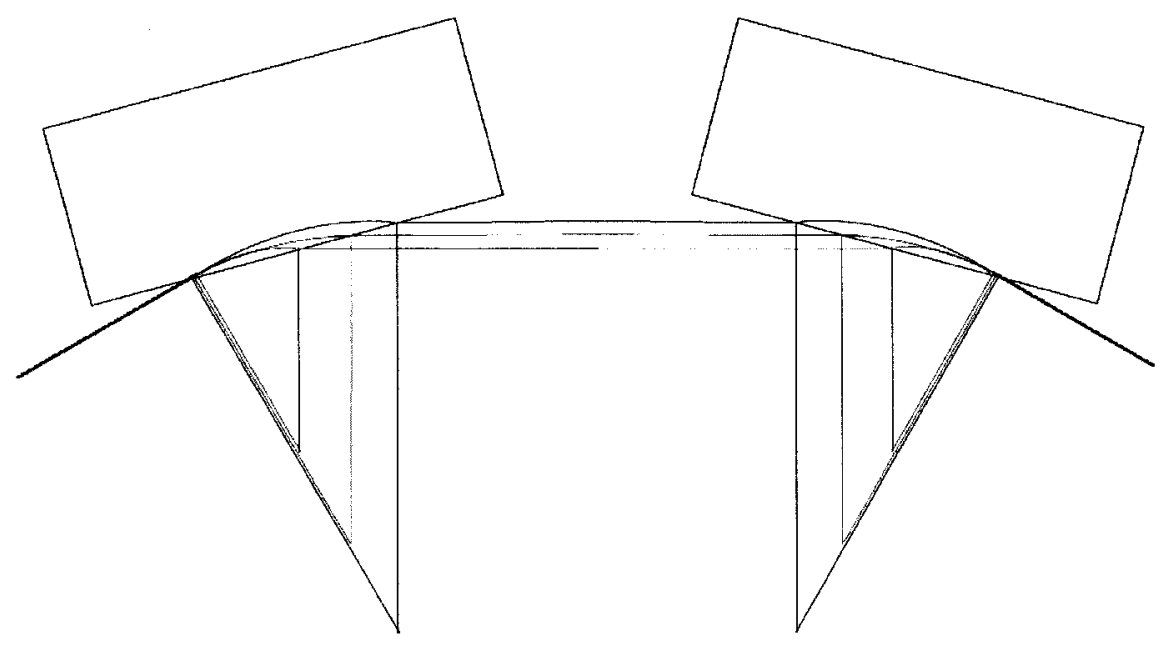

(a)

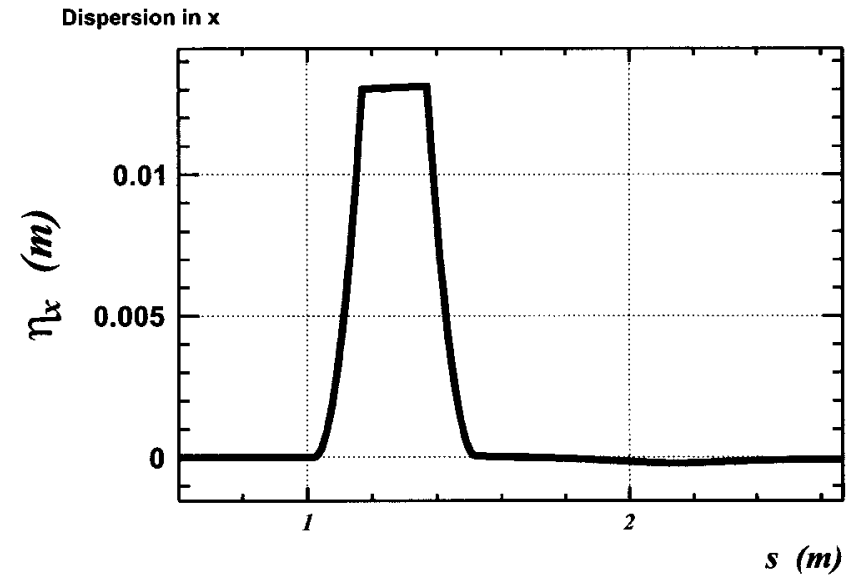

(b)

FIG. 80: (a) The novel design by using two back to back microtron dipoles. (b) The dispersion $\eta_{x}$ as a function of the central orbit is shown. 


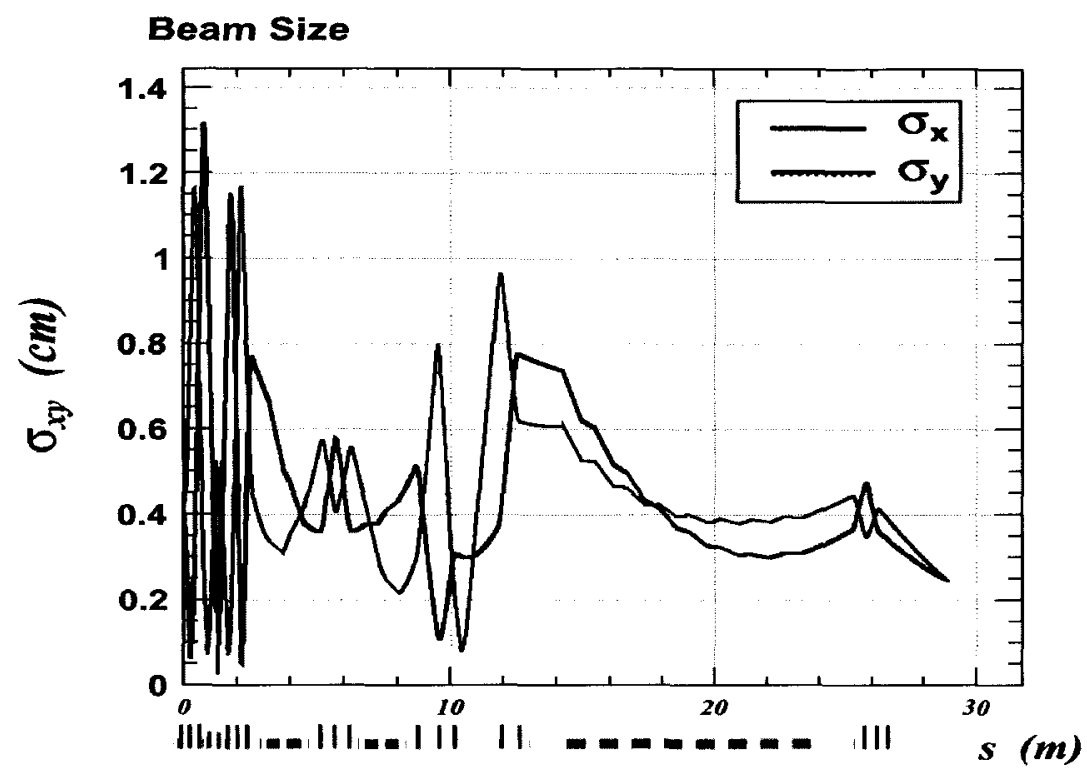

(a)

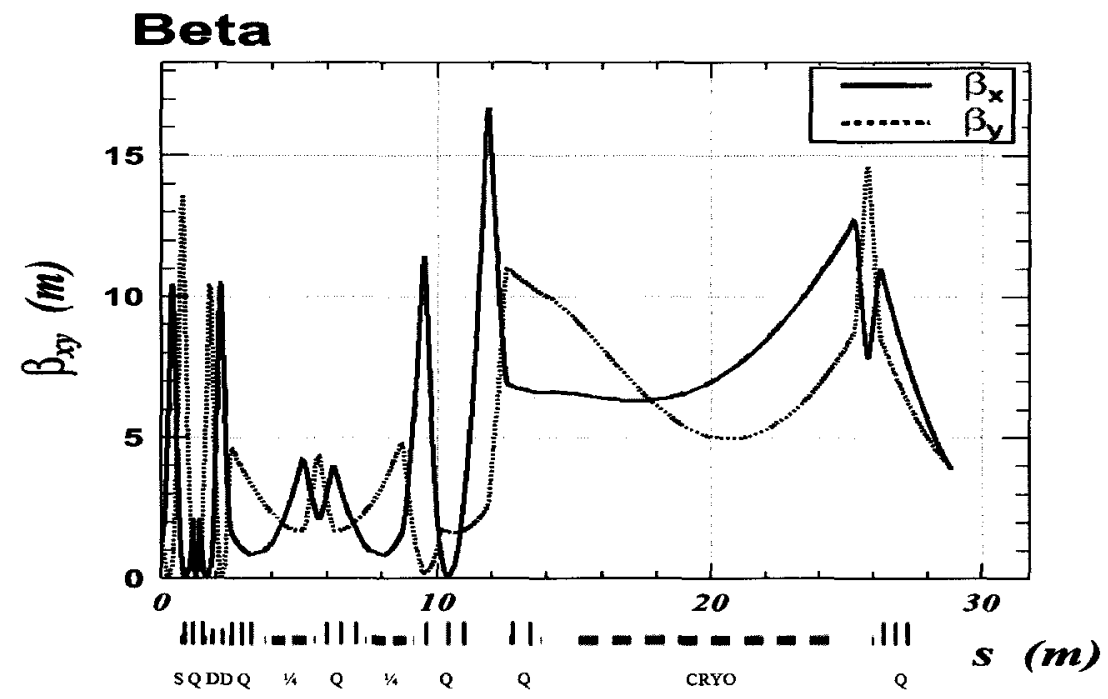

(b)

FIG. 81: (a) The 1- $\sigma$ beam size of the positron beam as a function of the central orbit in the microtron dipole lattice is plotted. The solid line is $\sigma_{x}$ and dashed line is $\sigma_{y}$. (b) The twiss $\beta_{x y}$ functions for the same lattice are plotted where the solid line is $\beta_{x}$ and dashed line is $\beta_{y}$. The boxes under the horizontal axis show the location of the magnets, where the solenoid (S), dipoles (D), quadrupoles (Q), quarter cryomodules (1/4) and full cryomodule (CRYO). 
TABLE 17: Lattice description of the Microtron Dipole configuration. The dipole field values of the microtrons are: $\mathrm{B} 01=0.58218 k G, \mathrm{~B} 02=0.58218 k G$. Solenoid $\mathrm{C} 01$ has a field value $\mathrm{B}=9.8 k G$. Initial geometrical emittances given as input are $\varepsilon_{x y}=5.8 \mathrm{~mm}$.mrad at $p\left(e^{+}\right)=15 \mathrm{MeV} / \mathrm{c}$.

\begin{tabular}{|c|c|c|c|c|c|c|c|c|c|}
\hline \hline $\mathbf{N}$ & Name & Type & $\begin{array}{c}\mathbf{S} \\
{[\mathbf{c m}]}\end{array}$ & $\begin{array}{c}\mathbf{L} \\
{[\mathbf{c m}]}\end{array}$ & $\begin{array}{c}\mathbf{G} \\
{[\mathbf{k G} / \mathbf{c m}]}\end{array}$ & $\begin{array}{c}\beta_{x} \\
{[\mathbf{c m}]}\end{array}$ & $\begin{array}{c}\alpha_{x} \\
-\end{array}$ & $\begin{array}{c}\beta_{y} \\
{[\mathbf{c m}]}\end{array}$ & $\begin{array}{c}\alpha_{y} \\
-\end{array}$ \\
\hline \hline 0 & START & Target & 0 & & & 0.3 & -0.1 & 0.3 & -0.1 \\
\hline 1 & C01 & Solenoid & 16 & 16.0 & & 344 & -0.1 & 336 & -0.1 \\
\hline 2 & oDAB0 & Drift & 22 & 6.0 & & 344 & -0.1 & 337 & -0.1 \\
\hline 3 & HC & Collimator & 22 & 0 & & 344 & -0.1 & 337 & -0.1 \\
\hline 4 & oD00 & Drift & 25 & 3 & & 345 & -0.1 & 338 & -0.1 \\
\hline 5 & iIPM0 & BPM & 25 & 0 & & 345 & -0.1 & 338 & -0.1 \\
\hline 6 & oD00 & Drift & 28 & 3 & & 345 & -0.1 & 338 & -0.1 \\
\hline 7 & qA1 & Quad & 43 & 15 & -0.156 & 654 & -25.1 & 154 & 9.4 \\
\hline 8 & oDA1 & Drift & 61 & 18 & & 1872 & -42.5 & 4 & -1.0 \\
\hline 9 & qA2 & Quad & 76 & 15 & 0.165 & 1698 & 51.1 & 221 & -16.9 \\
\hline 10 & oDA2 & Drift & 95 & 19.0 & & 311 & 21.9 & 1328 & -41.4 \\
\hline 11 & qA3 & Quad & 110 & 15.0 & -0.175 & 16 & 2.7 & 1441 & 36.0 \\
\hline 12 & oD00 & Drift & 113 & 3.0 & & 5 & 1.2 & 1233 & 33.3 \\
\hline 13 & ilPM1 & BPM & 113 & 0.0 & & 5 & 1.2 & 1233 & 33.3 \\
\hline 14 & oDA3 & Drift & 127 & 14.0 & & 72 & -6.0 & 477 & 20.7 \\
\hline 15 & HC & Collimator & 127 & 0.0 & & 72 & -6.0 & 477 & 20.7 \\
\hline 16 & oD00 & Drift & 130 & 3.0 & & 113 & -7.5 & 361 & 18.0 \\
\hline 17 & g01 & Edge & 130 & 0.0 & & 113 & -7.7 & 361 & 18.7 \\
\hline 18 & B01 & Dipole & 145 & 15.0 & & 458 & -15.0 & 19 & 4.1 \\
\hline 19 & g02 & Edge & 145 & 0.0 & & 458 & 45.8 & 19 & 1.6 \\
\hline 20 & oDL0 & Drift & 165 & 20.0 & & 461 & -46.0 & 32 & -2.3 \\
\hline 21 & g02 & Edge & 165 & 0.0 & & 461 & 15.3 & 32 & -6.6 \\
\hline 22 & B01 & Dipole & 180 & 15.0 & & 111 & 7.8 & 540 & -27.3 \\
\hline & & & Continued on Next Page.. & & & \\
\hline
\end{tabular}


TABLE 17 - Continued

\begin{tabular}{|c|c|c|c|c|c|c|c|c|c|}
\hline $\mathbf{N}$ & Name & Type & $\begin{array}{c}\mathrm{S} \\
{[\mathrm{cm}]}\end{array}$ & $\begin{array}{c}\mathbf{L} \\
{[\mathrm{cm}]}\end{array}$ & $\begin{array}{c}\mathrm{G} \\
{[\mathrm{kG} / \mathrm{cm}]}\end{array}$ & $\begin{array}{c}\beta_{x} \\
{[\mathrm{~cm}]}\end{array}$ & $\begin{array}{r}\alpha_{x} \\
-\end{array}$ & $\begin{array}{c}\beta_{y} \\
{[\mathrm{~cm}]}\end{array}$ & $\begin{array}{c}\alpha_{y} \\
-\end{array}$ \\
\hline 23 & g01 & Edge & 180 & 0.0 & & 111 & 7.6 & 540 & -26.3 \\
\hline 24 & oDB0 & Drift & 196 & 16.0 & & 3 & -0.8 & 1708 & -46.7 \\
\hline 25 & $\mathrm{HC}$ & Collimator & 196 & 0.0 & & 3 & -0.8 & 1708 & -46.7 \\
\hline 26 & oD00 & Drift & 199 & 3.0 & & 13 & -2.4 & 2000 & -50.6 \\
\hline 27 & iIPM2 & BPM & 199 & 0.0 & & 13 & -2.4 & 2000 & -50.6 \\
\hline 28 & qB1 & Quad & 214 & 15.0 & -0.174 & 292 & -20.9 & 1864 & 57.1 \\
\hline 29 & oDB1 & Drift & 234 & 20.0 & & 1726 & -50.8 & 280 & 22.1 \\
\hline 30 & $\mathrm{qB} 2$ & Quad & 249 & 15.0 & 0.162 & 1893 & 42.5 & 2 & 0.7 \\
\hline 31 & oDB2 & Drift & 269 & 20.0 & & 575 & 23.4 & 313 & -16.3 \\
\hline 32 & qB3 & Quad & 284 & 15.0 & -0.156 & 271 & 1.4 & 582 & 2.7 \\
\hline 33 & oDB3 & Drift & 324 & 40.0 & & 179 & 0.9 & 387 & 2.1 \\
\hline 34 & iIPM3 & $\mathrm{BPM}$ & 324 & 0.0 & & 179 & 0.9 & 387 & 2.1 \\
\hline 35 & oD1006 & Drift & 349 & 25.0 & & 139 & 0.7 & 288 & 1.8 \\
\hline 36 & aC50-1 & $\mathrm{RF}$ & 399 & 50.0 & & 94 & 0.2 & 140 & 1.1 \\
\hline 37 & oD1006 & Drift & 424 & 25.0 & & 90 & -0.1 & 93 & 0.7 \\
\hline 38 & $\mathrm{aC} 50-1$ & $\mathrm{RF}$ & 474 & 50.0 & & 124 & -0.6 & 60 & -0.1 \\
\hline 39 & oD1006 & Drift & 499 & 25.0 & & 160 & -0.9 & 74 & -0.5 \\
\hline 40 & iIPM4 & BPM & 499 & 0.0 & & 160 & -0.9 & 74 & -0.5 \\
\hline 41 & oDC0 & Drift & 537 & 38.0 & & 241 & -1.3 & 136 & -1.1 \\
\hline 42 & $\mathrm{qC} 1$ & Quad & 552 & 15.0 & 0.07 & 234 & 1.7 & 205 & -3.8 \\
\hline 43 & oDC1 & Drift & 589 & 37.0 & & 131 & 1.1 & 587 & -6.6 \\
\hline 44 & $\mathrm{qC} 2$ & Quad & 604 & 15.0 & -0.119 & 142 & -1.9 & 591 & 6.3 \\
\hline 45 & oDC2 & Drift & 643 & 39.0 & & 337 & -3.1 & 203 & 3.6 \\
\hline 46 & $\mathrm{qC} 3$ & Quad & 658 & 15.0 & 0.081 & 356 & 2.0 & 143 & 0.7 \\
\hline 47 & oDC3 & Drift & 683 & 25.0 & & 266 & 1.6 & 114 & 0.4 \\
\hline 48 & iIPM5 & $\mathrm{BPM}$ & 683 & 0.0 & & 266 & 1.6 & 114 & 0.4 \\
\hline 49 & oD1006 & Drift & 708 & 25.0 & & 194 & 1.3 & 98 & 0.2 \\
\hline 50 & $\mathrm{aC} 50-2$ & $\mathrm{RF}$ & 758 & 50.0 & & 99 & 0.6 & 105 & -0.3 \\
\hline 51 & oD1006 & Drift & 783 & 25.0 & & 76 & 0.3 & 128 & -0.6 \\
\hline \multicolumn{10}{|c|}{ Continued on Next Page. } \\
\hline
\end{tabular}


TABLE 17 - Continued

\begin{tabular}{|c|c|c|c|c|c|c|c|c|c|}
\hline $\mathbf{N}$ & Name & Type & $\begin{array}{c}\mathrm{S} \\
{[\mathrm{cm}]} \\
\end{array}$ & $\begin{array}{c}\mathrm{L} \\
{[\mathrm{cm}]} \\
\end{array}$ & $\begin{array}{c}\mathrm{G} \\
{[\mathrm{kG} / \mathrm{cm}]} \\
\end{array}$ & $\begin{array}{c}\beta_{x} \\
{[\mathbf{c m}]}\end{array}$ & $\begin{array}{r}\alpha_{x} \\
- \\
\end{array}$ & $\begin{array}{c}\beta_{y} \\
{[\mathrm{~cm}]}\end{array}$ & $\begin{array}{c}\alpha_{y} \\
- \\
\end{array}$ \\
\hline 52 & aC50-2 & $\mathrm{RF}$ & 833 & 50.0 & & 84 & -0.4 & 210 & -1.1 \\
\hline 53 & oD1006 & Drift & 858 & 25.0 & & 114 & -0.8 & 270 & -1.3 \\
\hline 54 & iIPM6 & BPM & 858 & 0.0 & & 114 & -0.8 & 270 & -1.3 \\
\hline 55 & oDC0 & Drift & 896 & 38.0 & & 192 & -1.3 & 386 & -1.7 \\
\hline 56 & $\mathrm{qD} 1$ & Quad & 911 & 15.0 & -0.106 & 282 & -5.1 & 360 & 3.4 \\
\hline 57 & oDD1 & Drift & 975 & 64.0 & & 1322 & -11.2 & 70 & 1.2 \\
\hline 58 & $\mathrm{qD} 2$ & Quad & 990 & 15.0 & 0.167 & 1227 & 16.8 & 60 & -0.5 \\
\hline 59 & oDD2 & Drift & 1033 & 43.0 & & 209 & 6.9 & 136 & -1.3 \\
\hline 60 & qD3 & Quad & 1048 & 15.0 & -0.121 & 78 & 2.5 & 145 & 0.8 \\
\hline 61 & oDD3 & Drift & 1203 & 155.0 & & 1570 & -12.2 & 169 & -1.0 \\
\hline 62 & iIPM7 & BPM & 1203 & 0.0 & & 1570 & -12.2 & 169 & -1.0 \\
\hline 63 & oD00 & Drift & 1206 & 3.0 & & 1644 & -12.4 & 175 & -1.0 \\
\hline 64 & $\mathrm{qE} 1$ & Quad & 1221 & 15.0 & 0.108 & 1670 & 10.8 & 250 & -4.4 \\
\hline 65 & oDE1 & Drift & 1270 & 49.0 & & 779 & 7.4 & 873 & -8.3 \\
\hline 66 & $\mathrm{qE} 2$ & Quad & 1285 & 15.0 & -0.073 & 669 & 0.4 & 1001 & 0.2 \\
\hline 67 & oDE2 & Drift & 1315 & 30.0 & & 649 & 0.3 & 987 & 0.2 \\
\hline 68 & iIPM8 & $\mathrm{BPM}$ & 1315 & 0.0 & & 649 & 0.3 & 987 & 0.2 \\
\hline 69 & oD1005 & Drift & 1426 & 110.9 & & 603 & 0.1 & 953 & 0.1 \\
\hline 70 & oD1006 & Drift & 1451 & 25.0 & & 599 & 0.1 & 949 & 0.1 \\
\hline 71 & $\mathrm{aC} 100$ & $\mathrm{RF}$ & 1521 & 70.0 & & 578 & 0.2 & 915 & 0.4 \\
\hline 72 & oD1006 & Drift & 1546 & 25.0 & & 569 & 0.2 & 897 & 0.4 \\
\hline 73 & oD1006 & Drift & 1571 & 25.0 & & 562 & 0.1 & 880 & 0.3 \\
\hline 74 & $\mathrm{aC} 100$ & $\mathrm{RF}$ & 1641 & 70.0 & & 545 & 0.1 & 825 & 0.4 \\
\hline 75 & oD1006 & Drift & 1666 & 25.0 & & 540 & 0.1 & 804 & 0.4 \\
\hline 76 & oD1006 & Drift & 1691 & 25.0 & & 538 & 0.0 & 784 & 0.4 \\
\hline 77 & $\mathrm{aC} 100$ & $\mathrm{RF}$ & 1761 & 70.0 & & 536 & 0.0 & 730 & 0.4 \\
\hline 78 & oD1006 & Drift & 1786 & 25.0 & & 537 & -0.1 & 711 & 0.4 \\
\hline 79 & oD1006 & Drift & 1811 & 25.0 & & 541 & -0.1 & 694 & 0.3 \\
\hline 80 & $\mathrm{aC} 100$ & $\mathrm{RF}$ & 1881 & 70.0 & & 559 & -0.2 & 651 & 0.3 \\
\hline \multicolumn{10}{|c|}{ Continued on Next Page. . } \\
\hline
\end{tabular}


TABLE 17 - Continued

\begin{tabular}{|c|c|c|c|c|c|c|c|c|c|}
\hline \hline N & Name & Type & $\begin{array}{c}\mathbf{S} \\
{[\mathbf{c m}]}\end{array}$ & $\begin{array}{c}\mathbf{L} \\
{[\mathbf{c m}]}\end{array}$ & $\begin{array}{c}\mathbf{G} \\
{[\mathbf{k G} / \mathbf{c m}]}\end{array}$ & $\begin{array}{c}\beta_{x} \\
{[\mathbf{c m}]}\end{array}$ & $\begin{array}{c}\alpha_{x} \\
-\end{array}$ & $\begin{array}{c}\beta_{y} \\
{[\mathbf{c m}]}\end{array}$ & $\begin{array}{c}\alpha_{y} \\
-\end{array}$ \\
\hline \hline 81 & oD1006 & Drift & 1906 & 25.0 & & 569 & -0.2 & 638 & 0.2 \\
\hline 82 & oD1006 & Drift & 1931 & 25.0 & & 580 & -0.3 & 627 & 0.2 \\
\hline 83 & aC100 & RF & 2001 & 70.0 & & 621 & -0.3 & 602 & 0.1 \\
\hline 84 & oD1006 & Drift & 2026 & 25.0 & & 638 & -0.4 & 596 & 0.1 \\
\hline 85 & oD1006 & Drift & 2051 & 25.0 & & 658 & -0.4 & 592 & 0.1 \\
\hline 86 & aC100 & RF & 2121 & 70.0 & & 721 & -0.5 & 589 & 0.0 \\
\hline 87 & oD1006 & Drift & 2146 & 25.0 & & 746 & -0.5 & 591 & -0.1 \\
\hline 88 & oD1006 & Drift & 2171 & 25.0 & & 774 & -0.6 & 595 & -0.1 \\
\hline 89 & aC100 & RF & 2241 & 70.0 & & 859 & -0.6 & 616 & -0.2 \\
\hline 90 & oD1006 & Drift & 2266 & 25.0 & & 892 & -0.7 & 626 & -0.2 \\
\hline 91 & oD1006 & Drift & 2291 & 25.0 & & 927 & -0.7 & 638 & -0.3 \\
\hline 92 & aC100 & RF & 2361 & 70.0 & & 1034 & -0.8 & 682 & -0.4 \\
\hline 93 & oD1006 & Drift & 2386 & 25.0 & & 1074 & -0.8 & 701 & -0.4 \\
\hline 94 & oD1008 & Drift & 2522 & 135.9 & & 1329 & -1.0 & 839 & -0.6 \\
\hline 95 & iIPM9 & BPM & 2522 & 0.0 & & 1329 & -1.0 & 839 & -0.6 \\
\hline 96 & oDF0 & Drift & 2552 & 30.0 & & 1394 & -1.1 & 878 & -0.7 \\
\hline 97 & qF1 & Quad & 2567 & 15.0 & 0.168 & 1303 & 6.9 & 981 & -6.4 \\
\hline 98 & oDF1 & Drift & 2597 & 30.0 & & 921 & 5.8 & 1404 & -7.7 \\
\hline 99 & qF2 & Quad & 2612 & 15.0 & -0.316 & 899 & -4.3 & 1395 & 8.3 \\
\hline 100 & oDF2 & Drift & 2642 & 30.0 & & 1179 & -5.0 & 943 & 6.8 \\
\hline 101 & qF3 & Quad & 2657 & 15.0 & 0.163 & 1224 & 2.0 & 823 & 1.4 \\
\hline 102 & oDF3 & Drift & 2696 & 39.0 & & 1073 & 1.9 & 720 & 1.3 \\
\hline 103 & oD99 & Drift & 2805 & 109.0 & & 717 & 1.4 & 488 & 0.9 \\
\hline 104 & oD99 & Drift & 2914 & 109.0 & & 460 & 1.0 & 341 & 0.5 \\
\hline 105 & iIPM10 & BPM & 2914 & 0.0 & & 460 & 1.0 & 341 & 0.5 \\
\hline 106 & END & & 2914 & & & 460 & 1.0 & 341 & 0.5 \\
\hline & & & End of Table & & & & \\
\hline \hline
\end{tabular}




\section{Differential Pumping Cans}

The differential pumping (DP) cans are located right before and right after the cryomodule units at CEBAF. The DP cans are used to protect the quality of the vacuum in the $S R F$ cavities through isolating the high vacuum section from other sections. Three cans are positioned consecutively before and after the cryomodules in the accelerator sections. Each DP can is about $30 \mathrm{~cm}$ length. The inner radius is about $9 \mathrm{~mm}$, which is factor of two smaller than the vacuum pipe radius ( $\sim 18 \mathrm{~mm}$ ). As mentioned in Chapter III, our calculations concluded that because of the DP cans, the admittance values were small. As seen in beam profiles of positrons in CFM and 2-Dipole configurations, in Figs. 55 and 70 respectively, the full positron beam size reaches up to $30-50 \mathrm{~mm}$ before and after the cryomodules. In the simulations, the DP cans were not used. When placed right after and before the cryomodules, only $40 \%$ of the selected positrons make it until the end, to the NL connection point. For this reason, to protect the vacuum either DP cans must be replaced with an alternative option or they should be placed far from the cryomodules where the beam size is minimum. In Fig. 82, the cross section drawings of the DP cans are shown. In Fig. 83(a), the DP cans at the end of the injector at CEBAF are shown. In Fig. 83(b), the location of the connection point of the proposed positron injector and NL is shown. 


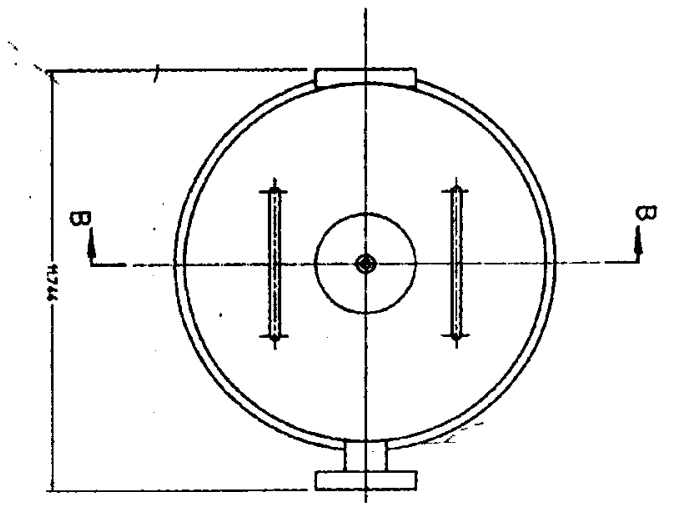

(a)

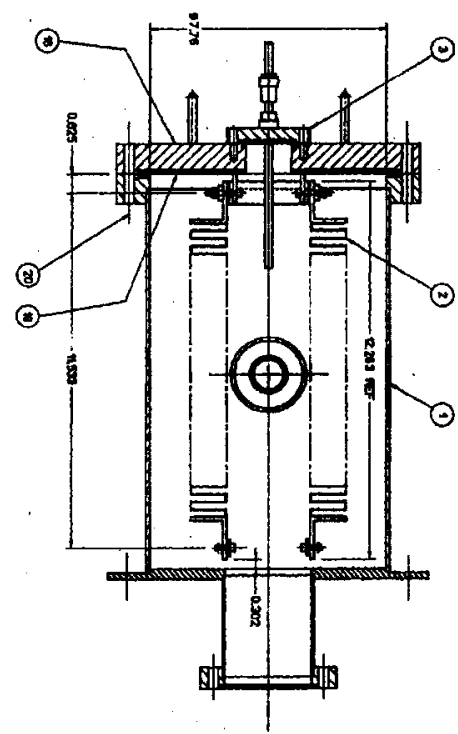

(b)

FIG. 82: (a) Top view of a single DP can. The beam direction is from bottom to top (or top to bottom). The flange-to-flange length of the DP can in the beam direction is 11.7 inches $=29.7 \mathrm{~cm}$. The pipe through the DP can is very tight for the positron beam. With the current beam initial parameters, simulation gives only about $40 \%$ of the initial number of positrons at the end of the full cryomodule. The DP can option should be replaced with another option allowing the usage of larger bore pipes. (b) Side view of a single DP can. The beam direction is through the page (out of the page to the reader). The inner middle circle is the pipe where beam passes through, the inside diameter is $\mathrm{D} \sim 0.75^{\prime \prime}=19.0 \mathrm{~mm}$. 


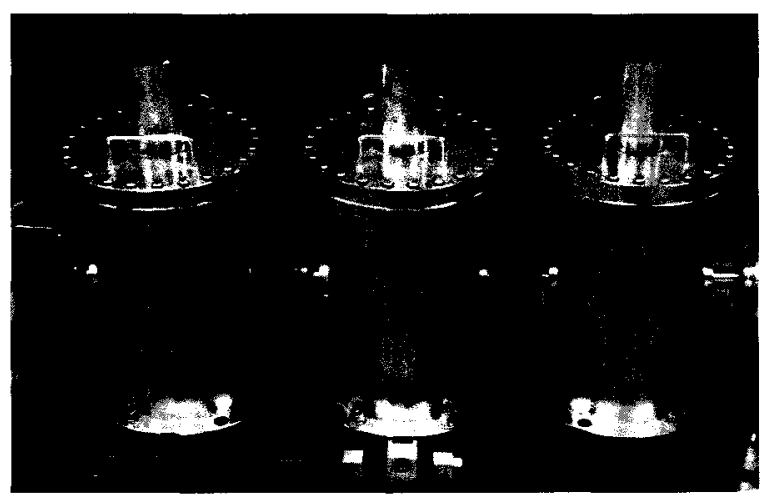

(a)

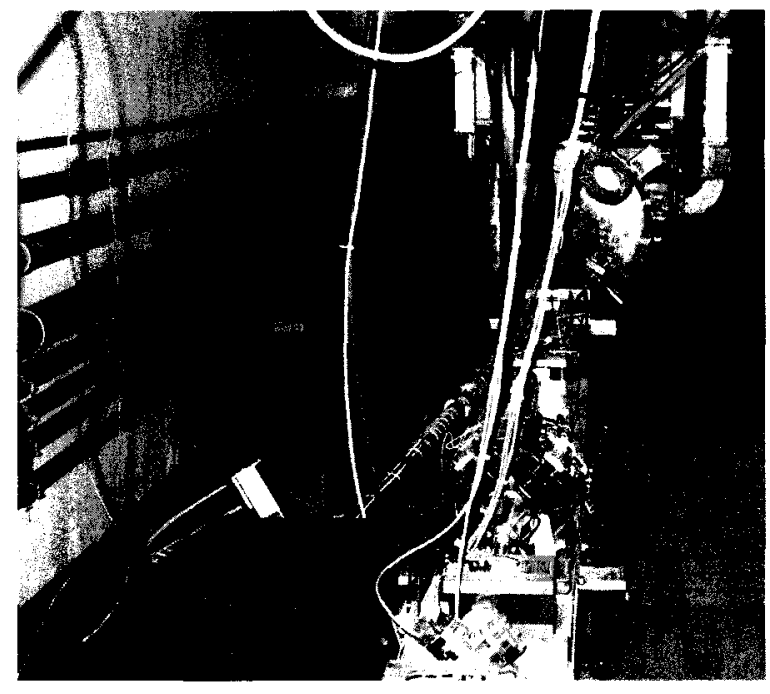

(b)

FIG. 83: (a) Three adjacently positioned DP cans immediately before the North Linac in the CEBAF. (b) The connection point between the proposed positron source and North Linac. There is about $1 \mathrm{~m}$ space between the wall and the centerline of the beam. This section has enough space to connect the positron source to the main linac. The only problem may be the first DP can right in front of the North Linac. This DP can should be considered to moved back so that the positron beam bypasses it. 


\section{CHAPTER V POWER AND RADIATION}

One of the hardest challenges of this unique $\mathrm{CW}$ positron source is the deposited power in the converter target and in the first few elements located in the radiation zone. First of all, there is a high risk of target melting, fracture and burning holes in the target during operation; secondly the radiation from the target poses high risk for the surrounding electronics and magnets as they will also be subject to a high dose; thirdly the entire beamline will be activated. These issues must be considered very carefully when the capture area and the target converter are engineered. Electrons lose energy via both ionization and bremsstrahlung radiation. When the average energy lost due this radiation and due to the ionization in the target is equal, this energy is called Critical Energy $\left(\mathrm{E}_{C}\right)$ [54]. This energy is given with:

$$
E_{C}=\frac{800}{Z+1.2}
$$

where here $\mathrm{Z}$ is the atomic number of the material. Radiation due to the bremsstrahlung losses start to dominate for $\mathrm{E}>\mathrm{E}_{C}$. For example, the critical energy for tungsten is $\mathrm{E}_{C}=10.2 \mathrm{MeV}$.

The particles propagate in the target in such a way that they continuously create photons, and then photons convert into $\mathrm{e}^{+}-\mathrm{e}^{-}$pairs. This process is called the cascade shower. The approximate propagation in the target is defined with a value called Radiation Length $\left(X_{0}\right)$. The radiation length is the mean thickness of materials for which an electron ends up with an energy a factor of $1 / e$ of its initial energy. The radiation length is related to the energy of the electron with the following:

$$
E=E_{0} e^{-\frac{x}{x_{0}}}
$$

where $E_{0}$ is the incoming electron energy and $E$ is the average energy of the electrons at a depth $X$ due to loss by bremsstrahlung. The probability of pair creation by a high energy photon $\left(>10 \mathrm{MeV}\right.$ ) in a thickness $X$ is $\frac{7}{9} X / X_{0}$ (if $X / X_{0} \ll 1$ ). Tungsten has a very short radiation length and a very high melting point making it a very good candidate for positron production. 


\section{V.1 POWER FLOW}

In this chapter, the amount of power deposited in the target and surrounding areas, as well as in the cryomodules are presented. Also existing and proposed target design options from other positron sources from the literature are introduced. The main source of the radiation is of course the converter target and the first few elements of the capture area. The incoming electron beam is over the photo-neutron activation limit causing neutrons to be emitted from the tungsten. The secondary particles emitted from the target also hit the iron and other elements in the capture zone and make them activated. As seen in Fig. 84, the number of neutrons/electrons increases with the increasing converter target thickness (e.g. Tungsten or Tantalum). At around $2 \mathrm{~mm}$ thickness of tungsten the efficiency is $\sim 8 \times 10^{-4}$ neutron per incoming electron. This may seem a small number but because this will be a continuous operation where the radioactivity builds up over time making it very hard to enter to the source area when needed. The bright side is, CEBAF was successfully built a MW beam dump sealing it from the outside without posing any harmful radiation.

In Fig. 85, the profile of the secondary electrons right after the target is shown. The incident electron energy is $126 \mathrm{MeV}$ impinging on a $2 \mathrm{~mm}$ tungsten converter. In Fig. 85(a), the momentum of the electron distribution shows almost a uniform distribution from 0 to $126 \mathrm{MeV}$. Fig 85 (b) shows the P- $\theta$ plot of these electrons right after the target. Although the number of low energy and high energy particles is almost even, it can be seen that the high energy electrons dominate the 0-degree line up to $\pm 100 \mathrm{mrad}\left(\sim \pm 5.7^{\circ}\right)$. Lower momenta electrons tend to spread all over the space from 0 to $\pm 1.5 \mathrm{rad}\left(\sim \pm 90^{\circ}\right)$.

The profile of the secondary $\gamma$ particles right after the target is shown in Fig 86 . In Fig 86(a), the momentum distribution of these photons shows a decline from the low energy band to the high energy band from 0 to $126 \mathrm{MeV}$. Fig $86(\mathrm{~b})$ shows the P- $\theta$ plot of these electrons. Lower energy photons tend to spread all over the space from 0 to $\pm 1.5 \mathrm{rad}\left(\sim \pm 90^{\circ}\right)$. The graph also shows that there are relatively very low number of high energy photons.

In all the design options provided in Chapter IV, the CFM, dipole and quadrupole locations as well as collimators are arranged such that positrons are deviated from the 0-degree line while high energy photons and electrons continue to follow 0-degree line and blocked by collimators. Low energy secondary particles at large angles are attenuated by collimators/absorbers. 


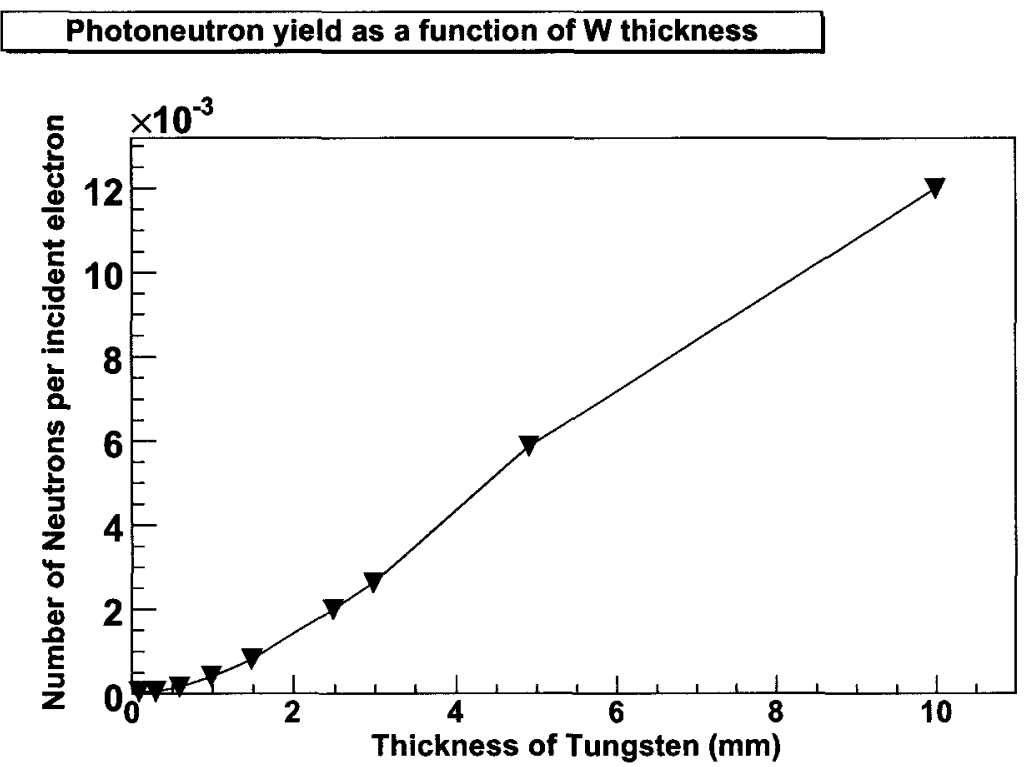

FIG. 84: Efficiency of the emitted neutron per incident electron $\left(p\left(\mathrm{e}^{-}\right)=126 \mathrm{MeV} / \mathrm{c}\right)$. The line is drawn to guide the eye only.

Table 18 shows the deposited power percentage w.r.t the incoming beam power at different locations. Here the calculation is made on the assumption of electron beam with $10 \mathrm{~mA}$ current at $126 \mathrm{MeV}$ hitting a $2 \mathrm{~mm}$ tungsten converter. This table is prepared by using the G4BEAMLINE simulation code. Significant amount of the deposited power comes from the bremsstrahlung photons and secondary electrons. The incoming electron beam carries $1.2 \mathrm{MW}$ beam power. Of this beam power; $\sim 60$ $\mathrm{kW}$ is deposited in the tungsten target; a lead collimator is positioned just after the target and there are other collimators in the radiation area, which in all of them 120 $\mathrm{kW}$ power is deposited. About $250 \mathrm{~kW}$ power is deposited in the solenoid coils itself. First few elements; such as CFMs and quadrupoles, dipoles right after the solenoid absorb about $200 \mathrm{~kW}$. About $4 \%$ of the beam power $(\sim 50 \mathrm{~kW})$ is backscattered from the target. In the cryomodules, positrons deposit very small amount of power, but this needs to be considered for a proper cryogenic operation as the cryogenics are sensitive to excessive heat load. Approximately 25 Watts is deposited in the 1 st $1 / 4$ cryomodule, 10 Watts is deposited in the 2 nd $1 / 4$ cryomodule and about 15 Watts is deposited in the full $\mathrm{C} 100$ cryomodule. One of the biggest concerns is that the capture area must be shielded radiation-proof since the remaining $500 \mathrm{~kW}$ is sprayed 


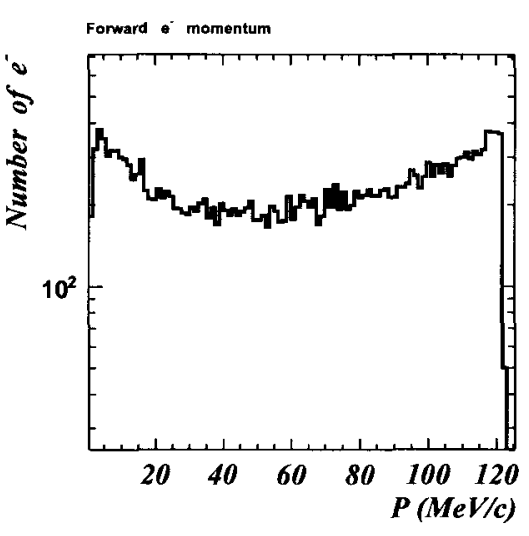

(a)

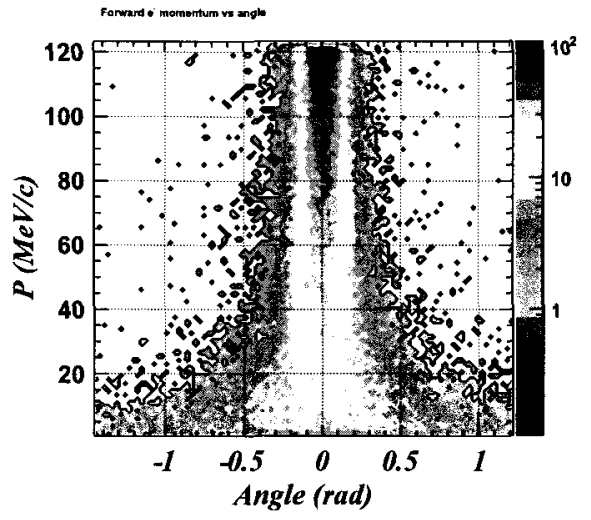

(b)

FIG. 85: (a) The momentum distribution of the electrons in the forward direction. Incident $p\left(\mathrm{e}^{-}\right)=126 \mathrm{MeV} / \mathrm{c}$ on a $2 \mathrm{~mm}$ tungsten. Forward direction means downstream of the tungsten converter. The momentum distribution shows a uniform distribution from 0 to $120 \mathrm{MeV}$. (b) The $p-\theta$ distribution of the forward electrons. The 0 -degree line from the target is dominated by high energy electrons $(\geq 60 \mathrm{MeV} / \mathrm{c})$

all over in this area. This area may be shielded with a layer of high atomic number material, such as lead or steel. The surrounding may also be covered by a low atomic material with high hydrogen content, such as concrete. The collimators can also be made by a lead followed by polyethylene [55]. This type of collimation and shielding attenuates the photons as well as neutrons and electrons (positrons).

The design options consider an electron beam spot size on the tungsten $\sigma \sim$ $100 \mu \mathrm{m}$. This small beam spot with the proposed beam energy makes an enormous hot spot with $\sim 10^{7} \mathrm{~W} / \mathrm{mm}^{2}$ on the target. Rastering both the electron beam and the collection optics up to a $5 \mathrm{~mm}$ square spot size will reduce the deposited power density to $240 \mathrm{~W} / \mathrm{mm}^{2}$. Conventional sources use either radiation cooling, water cooling or rotating target such as in SLAC as we investigated in Chapter II. There are a few possible options that go beyond the main objective of this dissertation. A promising solution is the ILC positron source proposal, which uses a powerful beam of photons on the target. 


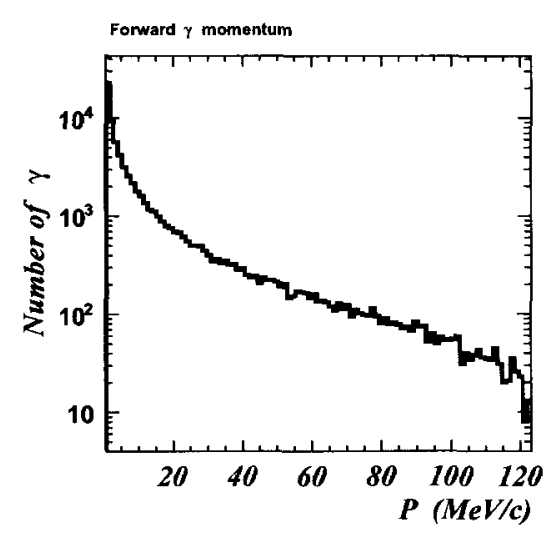

(a)

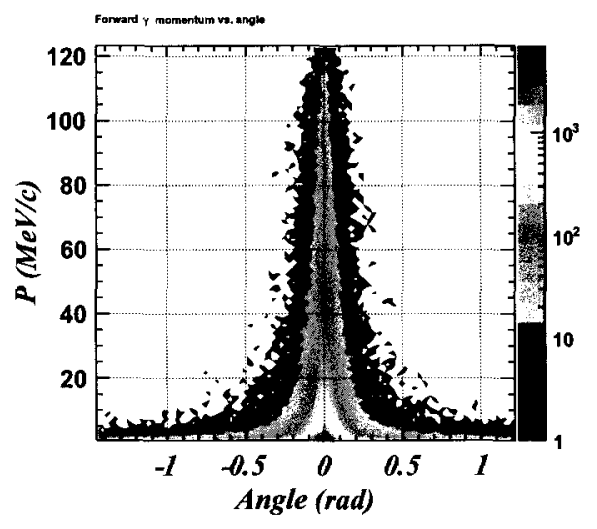

(b)

FIG. 86: (a) The momentum distribution profile of the secondary $\gamma$ particles right after the target. Same incident energy and target as in the previous figure. The momentum of the photon distribution (in log scale) shows a decline from the low energy band to the high energy band from 0 to $126 \mathrm{MeV}$. The number of high energy photons are in average about 100 times lower than the low energy photons, but when the power they are carrying is considered then the difference is not substantial and must be considered. (b) The $p-\theta$ plot of these photons. Lower energy photons tend to spread uniformly in the space from 0 to $\pm 1.5 \mathrm{rad}\left(\sim \pm 90^{\circ}\right)$.

\section{ILC Target}

As shown in Fig. 88, the ILC positron production target is a rotating wheel made of titanium alloy $90 \% \mathrm{Ti}-6 \% \mathrm{Al}-4 \% \mathrm{~V}$. As a part of two stage production process, firstly, $150 \mathrm{GeV}$ electrons pass through $200 \mathrm{~m}$ undulator magnet producing $10 \mathrm{MeV}$ photons, which the photon beam is incident on the rim of the spinning wheel. A wheel prototype has been built in the Daresbury laboratory [48]. The wheel has a $1 \mathrm{~m}$ diameter and $14 \mathrm{~mm}$ thickness (the projected design calls for a $2 \mathrm{~m}$ diameter wheel). The outer edge of the rim moves at $100 \mathrm{~m} / \mathrm{s}$. It has been estimated [56] that the photon beam power created via undulator is $\sim 130 \mathrm{~kW}$ on the target with each bunch of photons carrying a total energy of approximately $10 \mathrm{~J}$. Each photon bunch consists of order $10^{13}$ photons. The photon beam size is about 1-2 $\mathrm{mm}$. Of this photon beam power $\sim 10 \%$ is deposited in the titanium target $(\sim 13 \mathrm{~kW})$. The combination of wheel size and speed reduces the radiation damage, heating and the shock-stress in the wheel. In addition to the spinning wheel, the target mechanism is water cooled as well. 
TABLE 18: Deposited power for each region. About 5-6\% of the power is backscattered from the target. The remaining $500 \mathrm{~kW}$ power other than deposited is attenuated in the shielded radiation area. The equivalent power is calculated on the assumption of $126 \mathrm{MeV}$ electron beam with $10 \mathrm{~mA}$ current impinging on a $2 \mathrm{~mm}$ thick tungsten target, which is $1260 \mathrm{~kW}$ of incoming beam power.

\begin{tabular}{lccccc}
\hline \hline Element & \multicolumn{3}{c}{ Power Source } & \multicolumn{2}{c}{ Deposited Power } \\
& $\left(\mathbf{e}^{-}\right.$and $\left.\gamma\right)$ & $\mathbf{e}^{+}$ & $\begin{array}{c}\text { Distance } \\
(\%)\end{array}$ & $\mathbf{( k W )}$ & $\mathbf{m}$ \\
\hline Target & $\checkmark$ & $\checkmark$ & 5 & 60 & 0.0 \\
Solenoid & $\checkmark$ & $\checkmark$ & 21.0 & 250 & 0.16 \\
Collimators & $\checkmark$ & $\checkmark$ & 10.0 & 120 & 2.0 \\
Capture Area Magnets & $\checkmark$ & $\checkmark$ & 17.0 & 200 & 2.6 \\
1/4 Cryomodule-1 & & $\checkmark$ & $2.0 \times 10^{-3}$ & 0.025 & 4.0 \\
1/4 Cryomodule-2 & & $\checkmark$ & $9.0 \times 10^{-4}$ & 0.01 & 7.0 \\
Full Cryomodule & & $\checkmark$ & $1.2 \times 10^{-3}$ & 0.015 & 16.0 \\
\hline \hline
\end{tabular}

A liquid jet target which is made of mercury [46] or liquid $\mathrm{Bi}-\mathrm{Pb}$ [57] as shown in Fig. 87 has also been proposed as target alternatives for ILC. Detailed discussions and calculations can be found in these cited references and in Ref. [58, 45]. There are also very different ideas which are discussed in the same references for target design options such as suspension of tungsten(W) powder in liquid Li (Lithium) or InGa (Indium-Gallium) compound. In Ref. [57], liquid $\mathrm{Bi}-\mathrm{Pb}$ jet is recommended for CEBAF for its high vaporization point.

\section{Summary}

In summary for this chapter, there are a lot of great ideas in the literature and also a few $R \& D$ programs have been running for similar target designs. Whether it is possible to use a rotating target which may be coupled with a water or other cooling solution or not, the target design for such a high power $\mathrm{CW}$ source, it is required that proof-of-principle tests and extensive engineering work must be completed. It is very important making the target very reliable and easy to maintain. In ILC target design, the estimated power deposition in the target is about $10-13 \mathrm{~kW}$. In CEBAF positron source, we estimate that approximately $55-60 \mathrm{~kW}$ of the incoming $1.2 \mathrm{MW}$ beam 


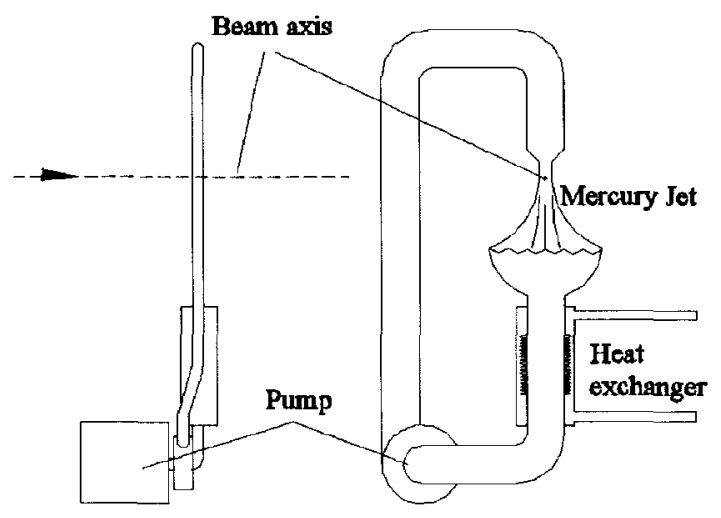

FIG. 87: An alternative liquid jet target for ILC positron source [47].

power is deposited in the $2 \mathrm{~mm}$ tungsten target. In addition to spinning the target wheel or using a liquid jet target, we can consider rastering the incoming electron beam and dipole components positioned immediately after the target as well. This may help us to change the hot spot locations with some frequency and increase the efficiency of the cooling process. These techniques may help us to sufficiently cool the target without sacrificing from the positron current.

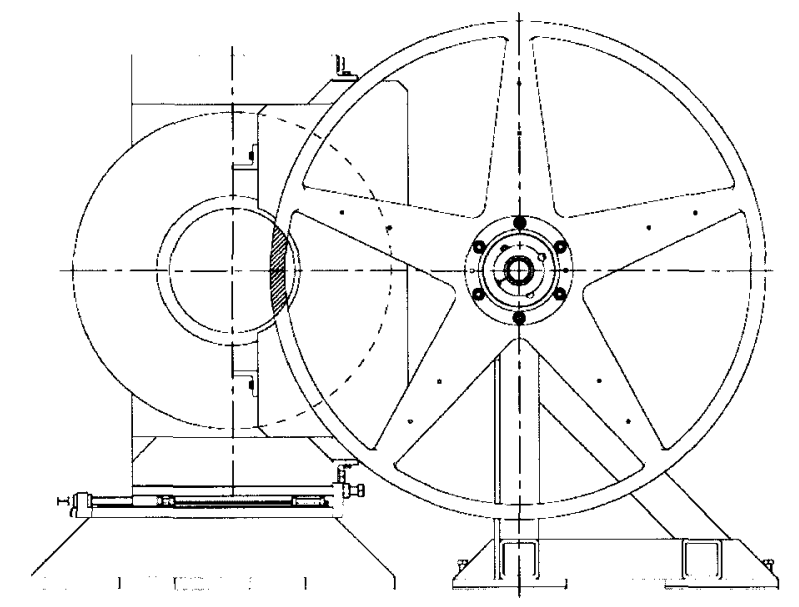

FIG. 88: Cross section of the proposed ILC rotating positron converter target assembly [48]. 


\section{CHAPTER VI SUMMARY AND CONCLUSION}

We have investigated the feasibility of a $\mathrm{CW}$ positron source for CEBAF. Three design options are considered as possible solutions for a positron injector. When investigating various positron sources in certain major laboratories, it is seen that all these accelerator based positron source have some common features. They use pulsed electron beam as the driving beam; they use solenoidal fields to capture the converted positrons; immediately following the capture they use normal-conducting (NC) RF section(s); to bunch and to reduce the emittance of the beam in all directions, they utilize large damping rings. Future projects investigate the possibility of directly using polarized photons to pair convert positrons, which the photons are created via undulators. For CEBAF design options some of these features are adopted. We have also seen that non-accelerator based sources such as $\beta^{+}$isotopes would not produce enough current for CEBAF.

The positron beam will not have the same quality as the electron beam. It will have significantly larger transverse and longitudinal emittance values. Admittance measurements of the CEBAF injector and ARC1 were made and the data were used to define the positron parameters used in the design studies. The admittance data are presented in Chapter III. It has been found that measured transverse geometrical admittance of the CEBAF injector is in close agreement with calculated values. The transverse geometrical admittance of the injector, 10 and $5 \mathrm{~mm} \cdot \mathrm{mrad}$ in horizontal and vertical respectively, are about 1000 times larger than present electron beam emittance in the injector. In addition to the transverse geometrical admittance, the longitudinal admittance of the ARC1 (the energy spread) were also measured. The fractional energy spread $\delta= \pm 3 \times 10^{-3}$ at $653 \mathrm{MeV}$ is the measured longitudinal admittance value. This spread value is by a factor of 30 larger than the electron beam energy spread currently measured in CEBAF. Although in our positron simulations we have used $\sigma_{p}=0.5 \mathrm{MeV} / \mathrm{c}$ which is $\delta=3.3 \%$ energy spread at $15 \mathrm{MeV} / \mathrm{c}$, this larger admittance value may give us more room to have much larger current for the positron beam. As it is seen that the phase space area of the positron beam will be much larger than the CEBAF electron beam. However, a numerical study [59] shows that if a positron beam which has these parameters is propagated until the final pass in the upgraded $12 \mathrm{GeV}$ CEBAF, the transverse emittance and the energy 
spread of the beam is on the order of a comparable size when the electron beam is propagated. This is mainly due to growth in the emittance of the electron beam by the synchrotron radiation in the $12 \mathrm{GeV}$ upgrade.

Measuring the admittance values of CEBAF injector provides the positron beam requirements needed for selecting and designing a positron source. In Chapter IV, by using Monte Carlo simulations we have numerically simulated the positron yield, and applied cuts to find the most efficient energy bin within this positron output. This positron selection process is done via a brightness filter, which as a result to get the maximum positron yield per CEBAF admittance within the bins of energy spectrum of the created positrons. A fairly broad peak between $\sim 15-25 \mathrm{MeV} / \mathrm{c}$ was found after the brightness selection. We selected $15 \pm 0.5 \mathrm{MeV} / \mathrm{c}$ positrons as the central momentum since a quarter wave transformer (QWT) solenoid is utilized as the first element to capture the targeted positrons. Due to the technical restrictions, normal-conducting QW'T is operational up to the required capture field in DC mode.

Due to the time structure restrictions of CEBAF, positrons must be immediately separated from other secondary particles and be sent into a super-conducting $R F$ cryomodule before the time spread grows larger than nominal RF bucket structure. There are three possible layouts presented; all differ in the separation process. In all designs, we foresee QWT solenoid as the first capture optics, followed by separation process and matching quadrupoles. This front end capture system is sealed inside a vault where the vault blocks the radiation from this section. This capture section is about $3 \mathrm{~m}$ long. Followed by this capture section, there are two quarter cryomodule units, which totally give $20 \mathrm{MV}$ to the positron beam, increasing the beam energy up to $35 \mathrm{MeV}$. Finally, a full C100 cryomodule is positioned after these two quarter cryomodules, which the selected positrons are brought up to the North Linac (NL) injection energy $126 \mathrm{MeV}$.

The positron brightness selection process within the transverse and longitudinal admittance values of CEBAF, results in a positron efficiency of $3.6 \times 10^{-4} \mathrm{e}^{+} / \mathrm{e}^{-}$. With $126 \mathrm{MeV} @ 10 \mathrm{~mA}$ incoming electron beam on a $2 \mathrm{~mm}$ tungsten converter gives $\sim 3.6 \mu \mathrm{A}$ positron current within the transverse and longitudinal admittance values of CEBAF, which positron momentum is $15 \pm 0.5 \mathrm{MeV} / \mathrm{c}$. Our assumption was based on focusing the incoming electron beam down to a $100 \mu \mathrm{m}$ sigma spot size on the tungsten converter. In the combined magnet function solution approximately $3 \mu \mathrm{A}$ of the selected positrons are transported to the connection point of the North Linac. 
In this solution, there are 3 CFMs, 14 quadrupoles, 2 quarter cryomodules and 1 full cryomodule in the design lattice. In the two-dipole solution $\sim 2.7 \mu \mathrm{A}$ make it to the NL connection point. For the microtron dipole configuration, the same $\mu \mathrm{A}$ positron current level is estimated. In terms of magnet count, the CFMs are replaced with regular quadrupoles in the two-dipole solution.

To increase the positron yield, fairly large angle and energy spread are selected from the emitted positrons. The key point in this selection process is that the phase space of these positrons must have the CEBAF admittance values at the NL injection point. The adiabatic damping of the geometrical emittance from the acceleration is used to decrease the emittance. The created positron beam at the target is gaussian type in time and transverse position, while the positron cut are of non-gaussian type (almost flat) in angle and energy (after the admittance cuts). Non-gaussianity in addition to the large angular and energy spread presents a big challenge when designing the optics system. The challenge starts at the solenoid, where the transverse coordinates are coupled. This coupling process with the help of the chromatic effects increases the transverse emittance of the positrons. Using skew quads, or a reverse solenoidal field only decouples the coordinates, but doesn't improve the emittance growth. In quadrupoles because of the chromatic effects coupled with the fringe fields non-linear terms are added and these effects also result in the emittance growth. To minimize the chromatic effects, the separation magnets (CFM and dipoles) are designed to be achromatic type lattices. This achromatic section is very compact. Sextupole magnets must be used in a continuous dispersive region to suppress the chromatic effects. Unfortunately, sextupoles add more non-linear geometric aberration when suppressing the chromatic aberrations. This results in more emittance growth. However, sextupoles may be used in the arcs where after many turns off-momentum positrons will be degraded due to chromatic effects.

Another challenge in this CEBAF positron source will be designing a durable converter target design. Numerical simulations estimate that about $5 \%$ of the incoming electron beam power is deposited in the converter tungsten. By using our assumption for the electron beam, which carries 1.2 MW power, then this will result $\sim 60 \mathrm{~kW}$ power deposition in the tungsten. As given in Chapter $\mathrm{V}$, there are many ideas but few $R \& D$ projects going regarding this issue. Two possible solutions are proposed: using a rotating target wheel or a liquid jet target. In any case, the massive incoming beam power concentrated in a $100 \mu \mathrm{m}$ sigma spot size will be a 
challenge of an $R \& D$ process. To overcome this we can raster the electron beam and raster the dipole elements after the target. If these options can not be achieved in a conclusive manner to get the proposed positron current of $3 \mu \mathrm{A}$, then the incoming beam current and target thickness must be decreased.

Other than the target, also the front-end magnets will have the largest amount of power deposition. Simulations estimate about $\sim 400 \mathrm{~kW}$ of the incoming electron beam power is deposited in the solenoid, magnets and collimators in total. The collimators (possibly $\mathrm{Pb}$ ) are placed after each magnet in the front-end optics system. Front end optics system must be in a sealed vault, because of the remaining $\sim 500$ $\mathrm{kW}$ power escapes off the target. As it can be seen, the radiation emitted from the target and surrounding elements pose a great risk. For this reason, we recommend building the CEBAF positron injector in a separate tunnel, which will be in parallel location of the present injector tunnel. The tunnel area is estimated to be $5 \times 30 \mathrm{~m}^{2}$. The electron beam is transported into this tunnel during positron operation, then positrons are captured and transported from this tunnel to the existing tunnel and injected into the NL at the right energy. This way the existing tunnel will be as safe as the present radiation levels mandates.

In conclusion, if after an $R \& D$ project the infeasibility of a $60 \mathrm{~kW}$ target becomes apparent, we may then revise the estimated figures by lowering the incoming electron beam current or converter thickness. With the assumptions we made, it is plausible to conclude that with the presented design solutions we can have a positron current of at least $650 \mathrm{nA}$ with target designs already tested for the ILC, and a current up to $3 \mu \mathrm{A}$ if higher power targets can be developed. 


\section{BIBLIOGRAPHY}

[1] W.J. Kossler, A.J. Greer and L.D. Hulett Jr., Nucl. Instrum. Methods Phys. Res., Sect. B 79, 345 (1993).

[2] C. Hyde-Wright et. al., Jefferson Lab Letter-Of-Intent $-e^{+} / e^{-}$Physics at Jefferson Lab, PAC17, February (2000).

[3] A. W. Thomas, in American Institute of Physics Conference Proceedings 1160, $3(2009)$.

[4] E. Wilson, An introduction to Particle Accelerators, (Oxford University Press, New York, 2001).

[5] K. Wille, The Physics of Particle Accelerators, (Oxford University Press, New York, 2005).

[6] H. Wiedemann, Particle Accelerator Physics: Volume I, (Springer, Berlin, 2007 , 3rd Ed.).

[7] S. Y. Lee, Accelerator Physics, (World Scientific Publishing Company, Singapore, 1994).

[8] A. Chao, Handbook of Accelerator Physics and Engineering, (World Scientific Publishing Company, Singapore, 2002, 2nd Ed.).

[9] H. Wiedemann, Particle Accelerator Physics: Volume II, (Springer, Berlin, 1995).

[10] M. Reiser, Theory and Design of Charged Particle Beams, (Willey, New York, 1994).

[11] A. L. Septier, Applied Charged Particle Optics. Part A. Advances in Electronics and Electron Physics Supplement 13A, (Academic Press Inc., New York,1980).

[12] A. L. Septier, Applied Charged Particle Optics. Part B. Advances in Electronics and Electron Physics Supplement 13B, (Academic Press Inc., New York,1980).

[13] E. D. Courant and H. S. Snyder, Ann. Phys 3, 1 (1958). 
[14] R. Chasman, G. K. Green, and E. M. Rowe, IEEE Trans. Nucl. Sci. 22, 1765 (1975).

[15] M. Giovannozzi, CAS2005 - CERN Accelerator School Lectures (2005).

[16] $12 \mathrm{GeV}$ Upgrade Advisory Committee, White Paper: The Science Driving the 12 GeV Upgrade of CEBAF, February (2001).

[17] R. Kazimi, C. K. Sinclair, and G. A. Krafft, in Proceedings of the 20th International Linac Conference, Monterey, California, 2000.

[18] V. Lebedev, OPTIM, Particle Tracking Code, FNAL.

[19] M. Borland, ELEGANT, Particle Tracking and Simulation Code, ANL.

[20] D. M. Kaplan and T. J. Roberts, in Proceedings of Particle Accelerator Conference PAC07, Albuquerque, New Mexico, 2007.

[21] S. Agostinelli et.al, Nucl. Instrum. Methods Phys. Res., Sect. A 506, 250 (2003).

[22] R. Ley, Hyperfine Interactions 109, 167 (1997).

[23] C. Hugenschmidt et al., Nucl. Instrum. Methods Phys. Res., Sect. B 221, 160 (2004).

[24] A. Hathaway et al., Nucl. Instrum. Methods Phys. Res., Sect. A 579, 538 (2007).

[25] A. van Veen et al., Applied Surface Science 116, 39 (1997).

[26] R. Erickson, SLC Design Handbook, Technical Report SLAC-R-714, 1984.

[27] PEP-II an asymmetric B factory, Technical Report SLAC-R-418, 1993.

[28] J. E. Clendenin, High-yield positron systems for linear colliders, Technical Report SLAC-PUB-4743, 1989.

[29] H. L. Lynch et. al., IEEE Trans. Nucl. Sci. 32, 1832 (1985).

[30] J. Corbett, Recent Improvements in the SLC Positron System Performance, Technical Report SLAC-PUB-5786, 1992.

[31] S. Ecklund et. al., Isochronous $180^{\circ}$ turns for the SLC positron system, in Proceedings of Particle Accelerator Conference, 1991. 
[32] S. Ecklund, The SLC positron source, Technical Report SLAC-PUB-4437, 1987.

[33] BEPCII Group, BEPCII Design Report, in Proceedings of IHEP, 2001.

[34] X. Shen, Nuclear Physics B - Proceedings Supplements 185, 134 (2008).

[35] G.-X. Pei, BEPCII injector design considerations, in Proceedings of 2nd Asian Particle Accelerator Conference APAC '01, Beijing, China, 2001.

[36] G.-X. Pei, Overview of BEPCII linac design, in Proceedings of LINAC '02, Gyeongju, S.Korea, 2002.

[37] S.-H. Wang et al., Chin. Phys. Lett. 25, 1636 (2008).

[38] J. Barley, V. Medjidzade, and A. Mikhailichenko, New positron source for CESR, Technical Report CBN-01-19, 2001.

[39] D. B. Fromowitz, Increasing the positron capture efficiency of the CESR linac injector, PhD thesis, UMI-99-67514.

[40] The VEPP-4 accelerating-storage complex, http://v4.inp.nsk.su/index.en.html.

[41] A. N. Skrinsky, Nucl. Instrum. Methods Phys. Res., Sect. A 598, 1 (2009).

[42] V. Smaluk et. al., Status of VEPP-4M collider at BINP, in Proceedings of RuPAC 2008, Zvenigorod, Russia, pages $79-81$.

[43] A. Mikhailichenko, ILC undulator based positron source, tests and simulations, in Proceedings of the 2007 IEEE Particle Accelerator Conference (PAC 07), 2007.

[44] I. Bailey et al., Development of a positron production target for the ILC positron source, Technical Report Cockroft-06-12, 2006.

[45] M. Kuriki, ILC Positron Source Based on Liquid Metal Target, in Proceedings of the 4th Annual Meeting of Particle Accelerator Society of Japan, Japan, page $682,2007$.

[46] A. A. Mikhailichenko, Liquid metal target for ILC, in Proceedings of the European Particle Accelerator Conference (EPAC 06), Edinburgh, Scotland, 2006. 
[47] A. A. Mikhailichenko, Positron source for ILC a perspective, Technical Report CBN06-1, 2006.

[48] I. R. Bailey et. al., A prototype target wheel for the ILC positron source, in Proceedings of the EPAC 08, pages 706-708, 2008.

[49] C. Amsler et al., Particle Data Group Physics Letters B667 (2008).

[50] R. Chehab, Positron Sources, Technical Report LALIRT-92-17, Orsay, France, 1992.

[51] S. Suhring, CEBAF Nomenclature Document (CND), Technical note, JLAB91-083, 1999.

[52] CEBAF Injector Schematic, Technical Report JLAB-SSCA03, Jefferson Lab, 2002.

[53] D. Douglas, A nearly isochronous arc with unlimited momentum acceptance, Technical Report JLAB-TN-02-020, 2002.

[54] S. M. Seltzer and M. J. Berger, Studies of Penetration of Charged Particles in Matters., (Nuclear Science Series-39, Nat. Academy of Sciences, Washington DC, 1964).

[55] S. H. Rokni, J. D. Cossairt, and J. C. Liu, Radiation Shielding at High-Energy Electron and Proton Accelerators, Technical report, 2007, SLAC-PUB-13033.

[56] J. A. Clarke et. al., The Design of the Positron Source for the International Linear Collider, in Proceedings of the 11th European Particle Accelerator Conference, EPAC'08, Genoa, Italy, 2008.

[57] A. Mikhailichenko, AIP Conf. Proc. 1160, 81 (2009).

[58] V. Belov, V. Karasyuk, V. Kobets, G. Kraynov, and P. Martishkin, NASA STI/Recon Technical Report N 2, 91491 (2002).

[59] A. P. Freyberger, AIP Conf. Proc. 1160, 101 (2009). 


\section{VITA}

Serkan Golge

Department of Physics

Old Dominion University

Norfolk, VA 23529

ACADEMIC PREPARATION

- Ph.D., Physics Department, Old Dominion University, Norfolk, Virginia, August, 2010

- M.S., Physics Department, Old Dominion University, Norfolk, Virginia, December, 2004

- B.S., Physics Department, Fatih University, Istanbul, Turkey, July, 2002

\section{TALKS \& CONFERENCES}

1. Invited Talk at International Workshop on Positrons at Jefferson Lab, Newport News, VA USA 25-27 March 2009

2. Particle Accelerator Conference in Albuquerque, New Mexico, USA 25-29 June 2007

3. International Accelerator School for Linear Colliders, Sokendai, Hayama, Japan 19-27 May 2006

\section{PUBLICATIONS}

1. S. Golge, C. E. Hyde, and A. Freyberger, AIP Conf. Proc. 1160, 109 (2009)

2. S. Golge, C. E. Hyde-Wright, and A. Freyberger, in Proceedings of PAC07, Albuquerque, New Mexico, June 2007

Typeset using IATEX. $_{\mathrm{E}}$. 